\title{
Combining click reactions for the one-pot synthesis of modular biomolecule mimetics
}

Anne Brinkø, ${ }^{[1]}$ Christian Risinger, ${ }^{[2]}$ Annie Lambert, ${ }^{[3]}$ Ola Blixt, ${ }^{[2]}$ Cyrille Grandjean ${ }^{[3]}$ and Henrik H. Jensen*[1]

${ }^{1}$ Department of Chemistry, Aarhus University, Langelandsgade 140, 8000 Aarhus C, Denmark.

${ }^{2}$ Department of Chemistry, Chemical Biology, University of Copenhagen, Thorvaldsensvej 40, 1871 Frederiksberg C, Denmark

${ }^{3}$ Faculté des Sciences et des Techniques, Unité Fonctionnalité et Ingénierie des Protéines (UFIP), Université de Nantes, UMR CNRS 6286, 2, rue de la Houssinière, BP92208, 44322 Nantes Cedex 3, France

\section{Table of Contents}

Table of Contents 1

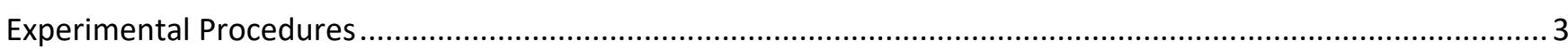

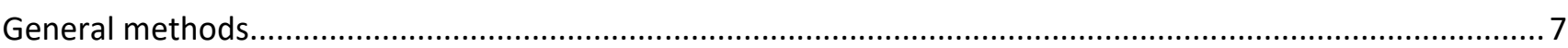

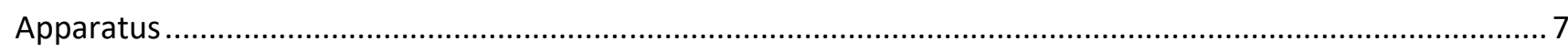

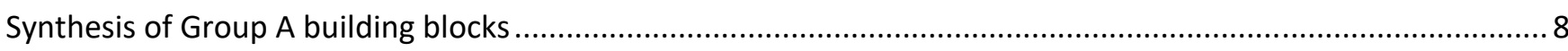

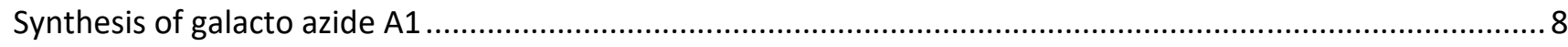

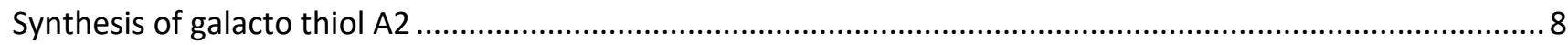

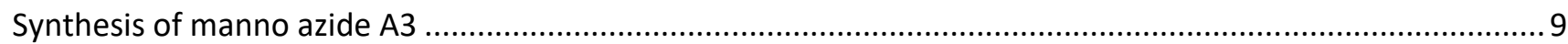

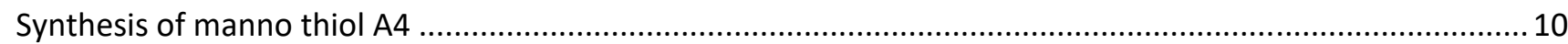

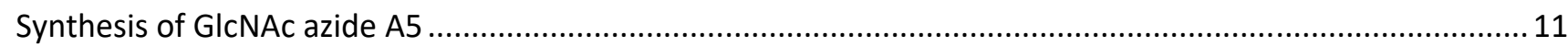

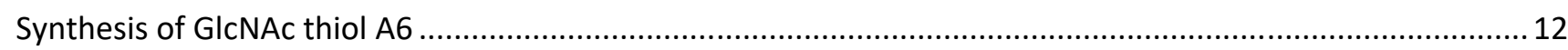

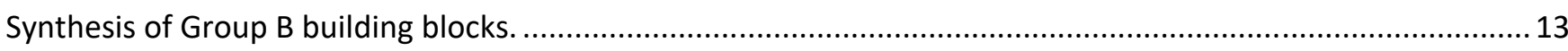

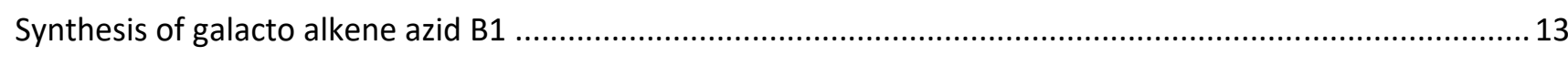

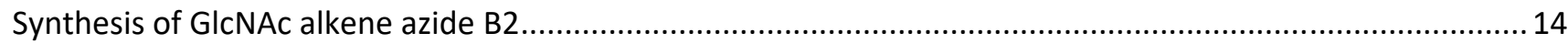

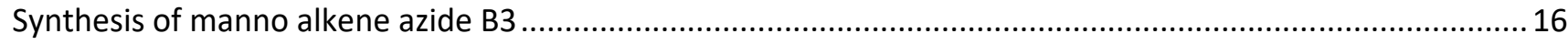

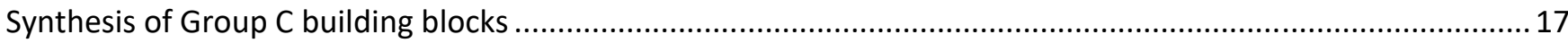

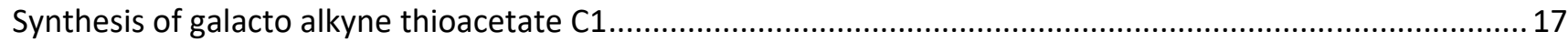

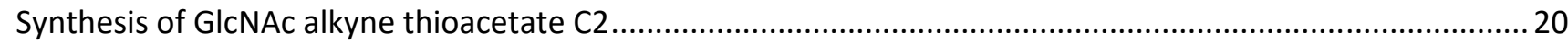

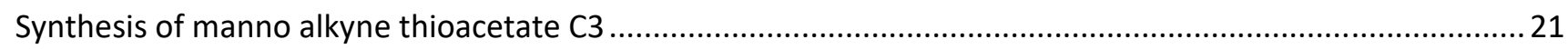

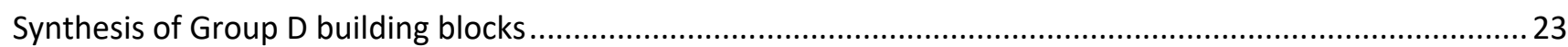




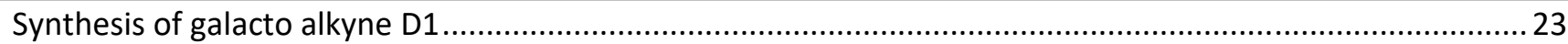

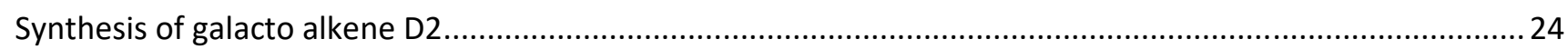

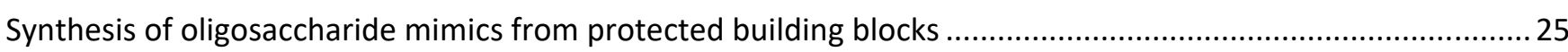

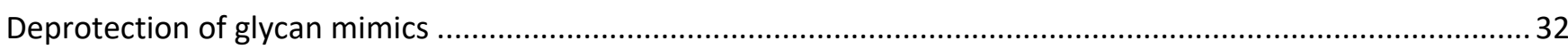

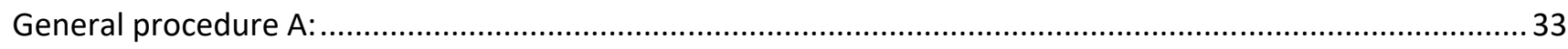

Synthesis of oligosaccharide mimic S34 from deprotected building blocks ....................................................... 37

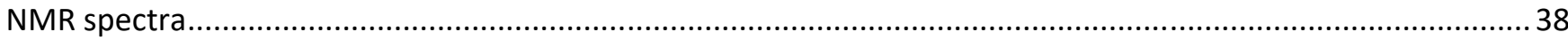

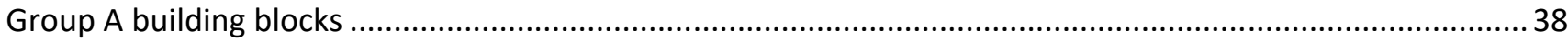

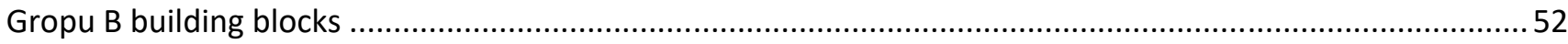

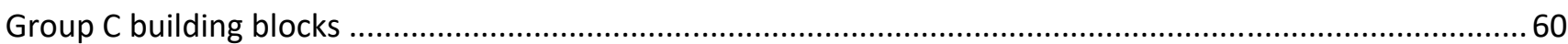

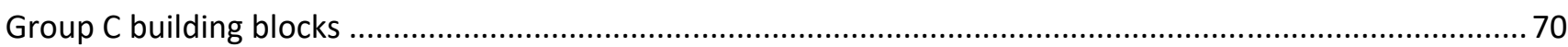

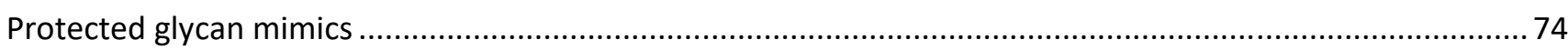

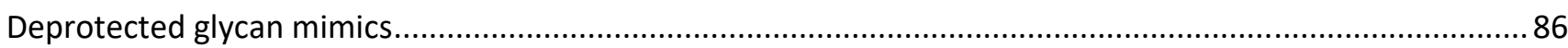

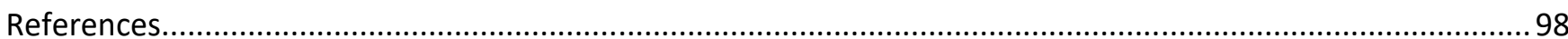




\section{Experimental Procedures}

\section{Immobilised Glycans}

The synthesized glycans were diluted in print buffer (300 mM phosphate buffer, $\mathrm{pH}$ 8.5) to the desired concentration. The glycans were printed by robotic pin deposition using a MicroGrid II arrayer (BioRobotics, Genomics Solutions, quilled pins, 250 $\mu \mathrm{m}$ pitch) onto N-hydroxysuccinimide (NHS)-activated glass array slides (SCHOTT NEXTERION® Slide H). The printed slides were placed in a high-humidity chamber for 1 hour. The remaining NHS groups were blocked by immersion in NHS blocking buffer (50 mM ethanolamine in $50 \mathrm{mM}$ borate buffer, $\mathrm{pH}$ 9.2) for $30 \mathrm{~min}$ just before use. The slides were incubated with Cy3conjugated lectins diluted 1:500 in PLI-P buffer for 1 hour or overnight with agitation at room temperature (RT) and washed three times with PBS. After the final washing step, the slides were rinsed in water and air-dried. The fluorescence signal was measured using a ScanArray 5000 (PerkinEImer) confocal scanner. ${ }^{1}$

\begin{tabular}{|c|c|c|}
\hline Position & Sequence & Concentration \\
\hline 1 & Gal-Gal (dA2-dD2) & $1000 \mu \mathrm{M}$ \\
\hline 2 & Gal-Gal (dA2-dD2) & $500 \mu \mathrm{M}$ \\
\hline 3 & Gal-Gal (dA2-dD2) & $100 \mu \mathrm{M}$ \\
\hline 4 & Gal-Gal (dA2-dD2) & $50 \mu \mathrm{M}$ \\
\hline 5 & Gal-Gal (dA2-dD2) & $10 \mu \mathrm{M}$ \\
\hline 6 & Gal-Gal (dA1-D1) & $1000 \mu \mathrm{M}$ \\
\hline 7 & Gal-Gal (dA1-D1) & $500 \mu \mathrm{M}$ \\
\hline 8 & Gal-Gal (dA1-D1) & $100 \mu \mathrm{M}$ \\
\hline 9 & Gal-Gal (dA1-D1) & $50 \mu \mathrm{M}$ \\
\hline 10 & Gal-Gal (dA1-D1) & $10 \mu \mathrm{M}$ \\
\hline 11 & Gal-Gal-Gal (dA2-dB1-dD1) & $1000 \mu \mathrm{M}$ \\
\hline 12 & Gal-Gal-Gal (dA2-dB1-dD1) & $500 \mu \mathrm{M}$ \\
\hline 13 & Gal-Gal-Gal (dA2-dB1-dD1) & $100 \mu \mathrm{M}$ \\
\hline 14 & Gal-Gal-Gal (dA2-dB1-dD1) & $50 \mu \mathrm{M}$ \\
\hline 15 & Gal-Gal-Gal (dA2-dB1-dD1) & $10 \mu \mathrm{M}$ \\
\hline 16 & Gal-Gal-Gal (dA1-dC1-dD2) & $1000 \mu \mathrm{M}$ \\
\hline 17 & Gal-Gal-Gal (dA1-dC1-dD2) & $500 \mu \mathrm{M}$ \\
\hline 18 & Gal-Gal-Gal (dA1-dC1-dD2) & $100 \mu \mathrm{M}$ \\
\hline 19 & Gal-Gal-Gal (dA1-dC1-dD2) & $50 \mu \mathrm{M}$ \\
\hline 20 & Gal-Gal-Gal (dA1-dC1-dD2) & $10 \mu \mathrm{M}$ \\
\hline 21 & Man-Gal-Gal (dA3-dC1-dD2) & $1000 \mu \mathrm{M}$ \\
\hline 22 & Man-Gal-Gal (dA3-dC1-dD2) & $500 \mu \mathrm{M}$ \\
\hline 23 & Man-Gal-Gal (dA3-dC1-dD2) & $100 \mu \mathrm{M}$ \\
\hline 24 & Man-Gal-Gal (dA3-dC1-dD2) & $50 \mu \mathrm{M}$ \\
\hline 25 & Man-Gal-Gal (dA3-dC1-dD2) & $10 \mu \mathrm{M}$ \\
\hline 26 & Gal-Gal-Gal-Gal (dA2-dB1-dC1-dD2) & $1000 \mu \mathrm{M}$ \\
\hline 27 & Gal-Gal-Gal-Gal (dA2-dB1-dC1-dD2) & $500 \mu \mathrm{M}$ \\
\hline 28 & Gal-Gal-Gal-Gal (dA2-dB1-dC1-dD2) & $100 \mu \mathrm{M}$ \\
\hline 29 & Gal-Gal-Gal-Gal (dA2-dB1-dC1-dD2) & $50 \mu \mathrm{M}$ \\
\hline 30 & Gal-Gal-Gal-Gal (dA2-dB1-dC1-dD2) & $10 \mu \mathrm{M}$ \\
\hline 31 & GlcNAc-Gal-Gal (dA6-dB1-dD1) & $1000 \mu \mathrm{M}$ \\
\hline 32 & GlcNAc-Gal-Gal (dA6-dB1-dD1) & $500 \mu \mathrm{M}$ \\
\hline 33 & GlcNAc-Gal-Gal (dA6-dB1-dD1) & $100 \mu \mathrm{M}$ \\
\hline 34 & GlcNAc-Gal-Gal (dA6-dB1-dD1) & $50 \mu \mathrm{M}$ \\
\hline 35 & GlcNAc-Gal-Gal (dA6-dB1-dD1) & $10 \mu \mathrm{M}$ \\
\hline 36 & Gal-GlcNAc-Gal (dA2-dB2-dD1) & $1000 \mu \mathrm{M}$ \\
\hline 37 & Gal-GlcNAc-Gal (dA2-dB2-dD1) & $500 \mu \mathrm{M}$ \\
\hline 38 & Gal-GlcNAc-Gal (dA2-dB2-dD1) & $100 \mu \mathrm{M}$ \\
\hline 39 & Gal-GlcNAc-Gal (dA2-dB2-dD1) & $50 \mu \mathrm{M}$ \\
\hline 40 & Gal-GlcNAc-Gal (dA2-dB2-dD1) & $10 \mu \mathrm{M}$ \\
\hline 41 & Gal-Gal-Gal-Gal (dA1-dC1-dB1-dD1) & $1000 \mu \mathrm{M}$ \\
\hline 42 & Gal-Gal-Gal-Gal (dA1-dC1-dB1-dD1) & $500 \mu \mathrm{M}$ \\
\hline
\end{tabular}




\begin{tabular}{|l|l|r|}
\hline 43 & Gal-Gal-Gal-Gal (dA1-dC1-dB1-dD1) & $100 \mu \mathrm{M}$ \\
\hline 44 & Gal-Gal-Gal-Gal (dA1-dC1-dB1-dD1) & $50 \mu \mathrm{M}$ \\
\hline 45 & Gal-Gal-Gal-Gal (dA1-dC1-dB1-dD1) & $10 \mu \mathrm{M}$ \\
\hline 46 & Man-Gal-Gal (dA4-dB1-dD1) & $1000 \mu \mathrm{M}$ \\
\hline 47 & Man-Gal-Gal (dA4-dB1-dD1) & $500 \mu \mathrm{M}$ \\
\hline 48 & Man-Gal-Gal (dA4-dB1-dD1) & $100 \mu \mathrm{M}$ \\
\hline 49 & Man-Gal-Gal (dA4-dB1-dD1) & $50 \mu \mathrm{M}$ \\
\hline 50 & Man-Gal-Gal (dA4-dB1-dD1) & $10 \mu \mathrm{M}$ \\
\hline 51 & Man-GlcNAc-Gal (dA4-dB2-dD1) & $1000 \mu \mathrm{M}$ \\
\hline 52 & Man-GlcNAc-Gal (dA4-dB2-dD1) & $500 \mu \mathrm{M}$ \\
\hline 53 & Man-GlcNAc-Gal (dA4-dB2-dD1) & $100 \mu \mathrm{M}$ \\
\hline 54 & Man-GlcNAc-Gal (dA4-dB2-dD1) & $50 \mu \mathrm{M}$ \\
\hline 55 & Man-GlcNAc-Gal (dA4-dB2-dD1) & $10 \mu \mathrm{M}$ \\
\hline 56 & Man-Man-Gal (dA4-dB3-dD1) & $1000 \mu \mathrm{M}$ \\
\hline 57 & Man-Man-Gal (dA4-dB3-dD1) & $500 \mu \mathrm{M}$ \\
\hline 58 & Man-Man-Gal (dA4-dB3-dD1) & $100 \mu \mathrm{M}$ \\
\hline 59 & Man-Man-Gal (dA4-dB3-dD1) & $50 \mu \mathrm{M}$ \\
\hline 60 & Man-Man-Gal (dA4-dB3-dD1) & $10 \mu \mathrm{M}$ \\
\hline 61 & GlcNAc-GlcNAc-Gal (dA6-dB2-dD1) & $1000 \mu \mathrm{M}$ \\
\hline 62 & GlcNAc-GlcNAc-Gal (dA6-dB2-dD1) & $500 \mu \mathrm{M}$ \\
\hline 63 & GlcNAc-GlcNAc-Gal (dA6-dB2-dD1) & $100 \mu \mathrm{M}$ \\
\hline 64 & GlcNAc-GlcNAc-Gal (dA6-dB2-dD1) & $50 \mu \mathrm{M}$ \\
\hline 65 & GlcNAc-GlcNAc-Gal (dA6-dB2-dD1) & $10 \mu \mathrm{M}$ \\
\hline 66 & Man-Gal-GlcNAc-Gal (dA3-dC1-dB2-dD1) & $1000 \mu \mathrm{M}$ \\
\hline 67 & Man-Gal-GlcNAc-Gal (dA3-dC1-dB2-dD1) & $100 \mu \mathrm{M}$ \\
\hline 68 & Man-Gal-GlcNAc-Gal (dA3-dC1-dB2-dD1) & $50 \mu \mathrm{M}$ \\
\hline 69 & Man-Gal-GlcNAc-Gal (dA3-dC1-dB2-dD1) & $10 \mu \mathrm{M}$ \\
\hline 70 & Man-Gal-GlcNAc-Gal (dA3-dC1-dB2-dD1) & $500 \mu \mathrm{M}$ \\
\hline 71 & GlcNAc (control) & $500 \mu \mathrm{M}$ \\
\hline 72 & GlcNAc (control) & \\
\hline
\end{tabular}

All compounds were printed in five different concentrations (1-0.01 mM) in triplicates. (see Table)

\section{WGA overnight incubation}

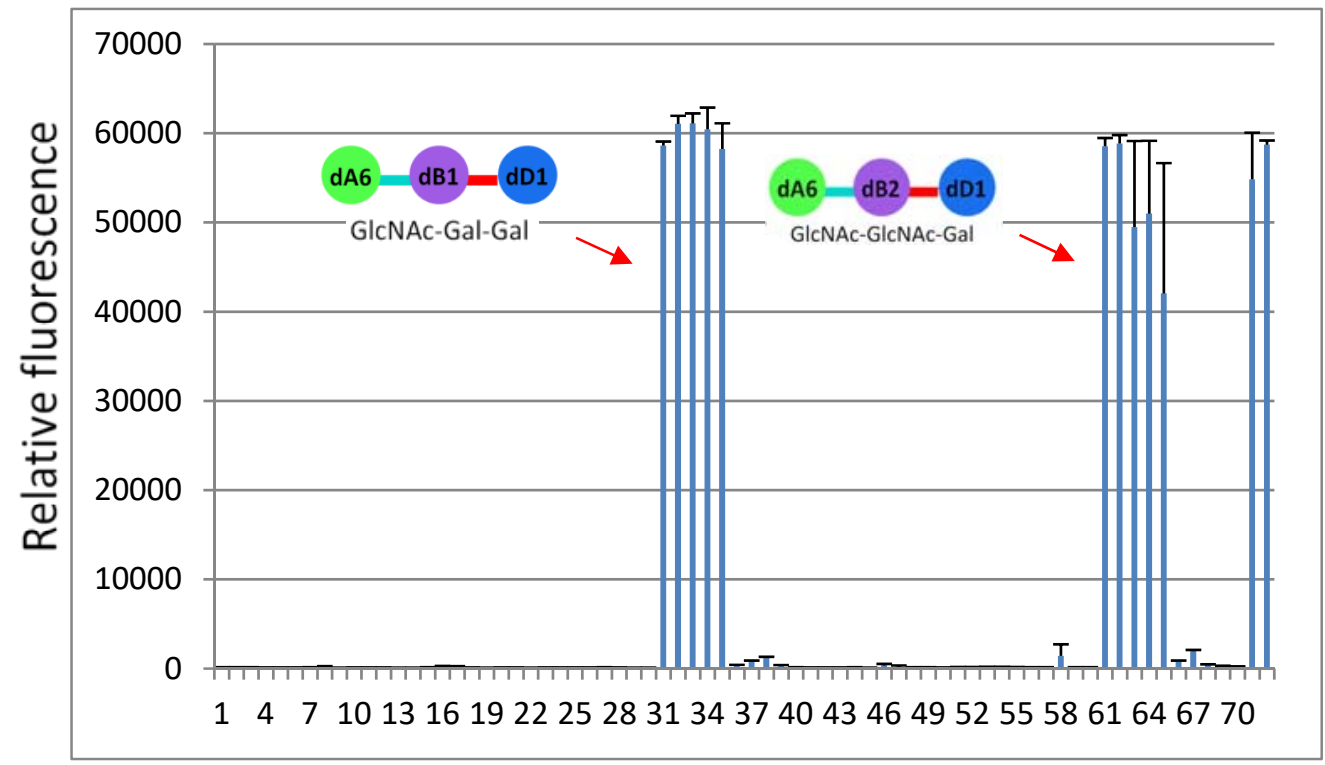

A strong binding to both glycan mimics containing terminal GlcNAc residues is observed. There is no significant difference in binding between the glycans containing either one or two GICNAc residues. 


\section{Con A overnight incubation}

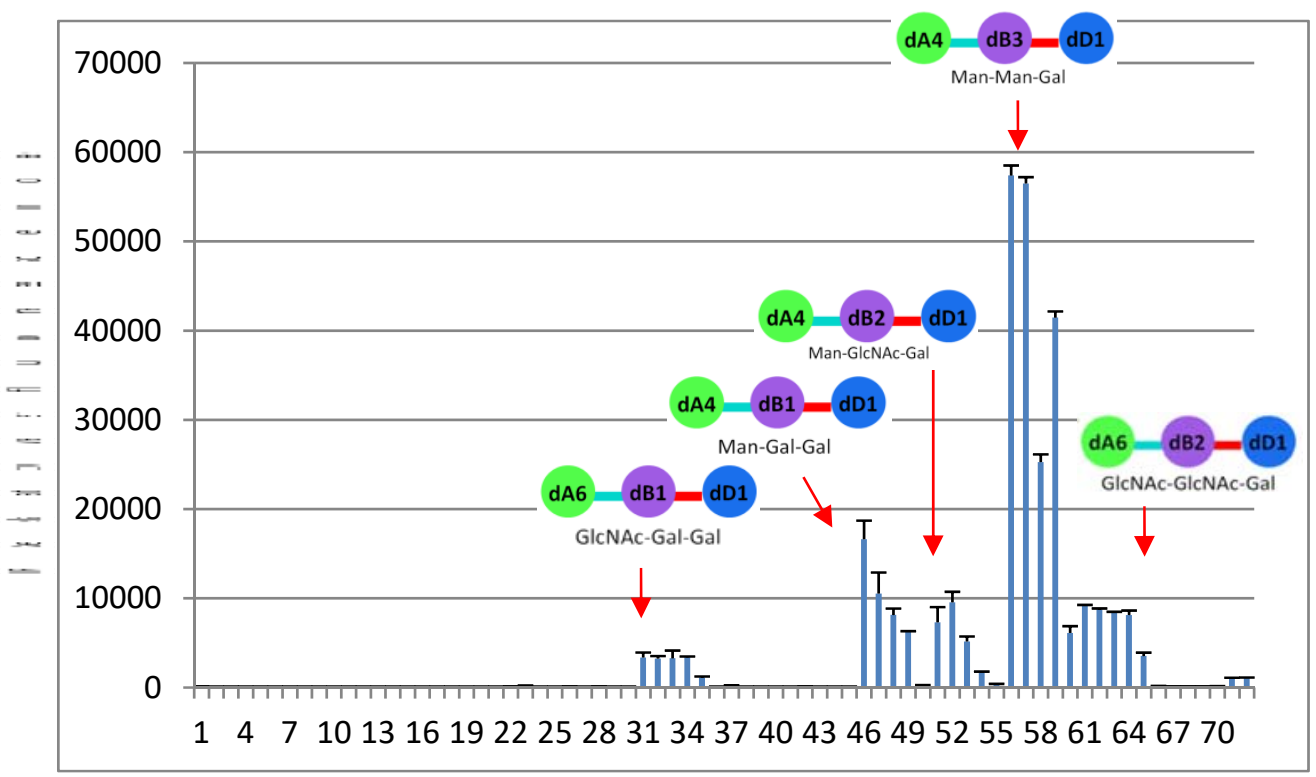

From 21-25 and 66-70 no binding is observed - Con A does not bind to mannose when it is linked with a triazole-linker.

\section{LEL overnight incubation}

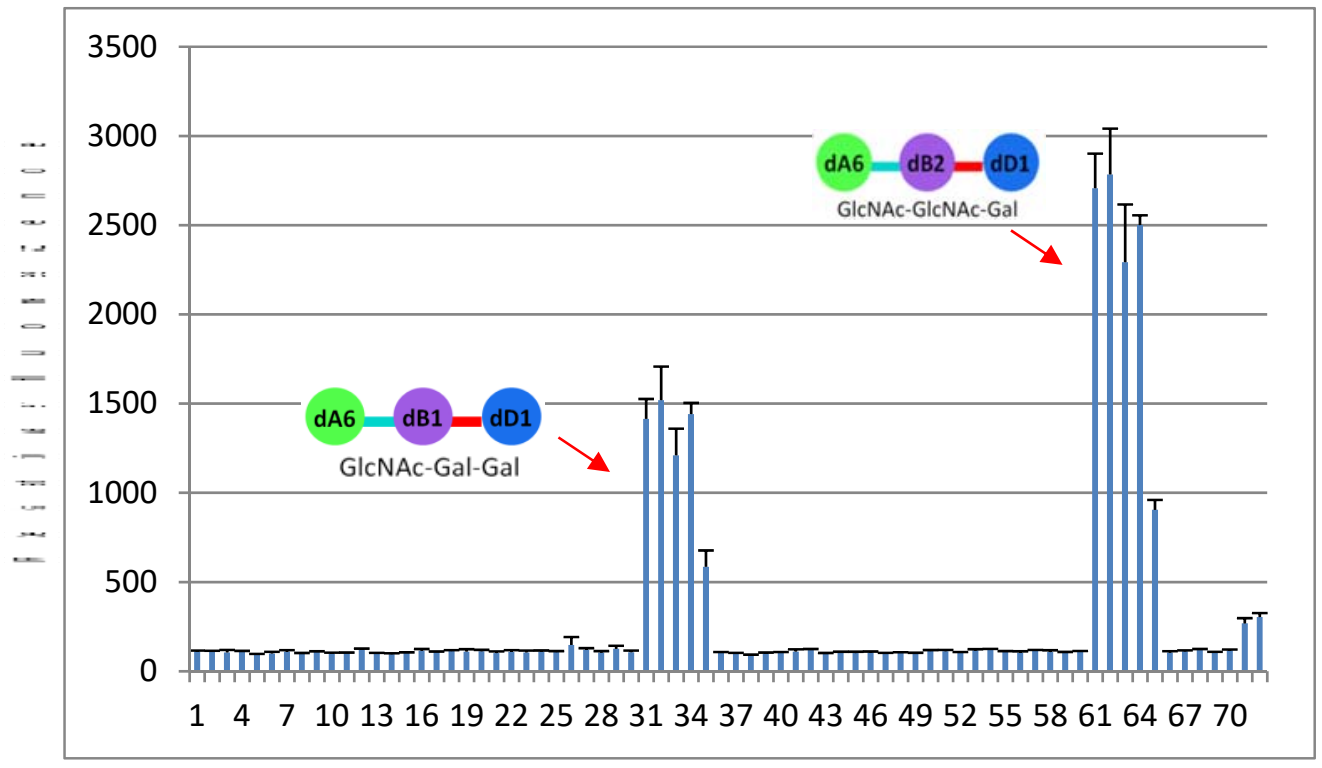

After 1 hour compounds with one or two GIcNAc residues are binding equally well, however, after incubation overnight, a slight difference can be observed, as expected since LEL is selective towards (GlcNAc) $2-4$. 


\section{Expression and purification of recombinant galectin-3}

Galectin3 human gene, cloned into pTRC vector, was kindly provided by Professor Pascal Reboul (IMoPA UMR7365 CNRSUniversity of Nancy-Lorraine, France). ${ }^{2}$

Production was carried out in E. coli BL21(DE3) strain transformed by constructions, overnight cultured in LB-ampicillin medium supplemented by $1 \mathrm{mM}$ of IPTG, at $20^{\circ} \mathrm{C}$. After protein extraction (avestin emulsiflex C5; 15000psi) in a $20 \mathrm{mM}$ phosphate$150 \mathrm{mM} \mathrm{NaCl}$ buffer pH7.35, (1 mM of PMSF, 2 mM EDTA, 4 mM DTT) purification is realized by column chromatography with a lactose agarose matrix (Sigma-Aldrich; L7634) at $4{ }^{\circ} \mathrm{C}$. Elution fractions (100mM of lactose) were measured by nanodrop at $280 \mathrm{~nm}$ and then extensively dialyzed (Spectra/Por Dialysis membrane tubing, 3.5kD) to eliminate lactose.

Fluorescence anisotropy

Fluorescence anisotropy measurements have been undertaken using galectin-3, known probe 2-[(fluoresceinyl)thioureido]ethyl 4-O-[3-O-(3-methoxybenzyl)- $\beta$-D-galactopyranosyl]- $\beta$-D-glucopyranoside ${ }^{3}$ and galectin inhibitors. All partners have been produced at UFIP (Nantes, France).

Every inhibitor was mixed at several concentrations $(0.05-1 \mu \mathrm{M}$ ) in phosphate buffer with a mixture of galectin (at a $100 \mathrm{nM}$ concentration)/fluorescein-labeled inhibitor (at a $2.2 \mu \mathrm{M}$ concentration) couple in a final volume of $200 \mu \mathrm{L}$ in phosphate buffer. The different solutions were incubated $1 \mathrm{~h}$ in 96 well microplates (Greiner Black FBottom). After incubation, fluorescence anisotropy was acquired using a microplate reader Infinite M1000 (TECAN, Suisse) and data were analysed with PRISM 6 software (GraphPad, USA) and Excel (Microsoft, USA). $K_{i}$ of each inhibitors was determined using the following equation,

$$
\log E C 50=\log \left(10^{\log K i} \times\left(1+\frac{C l}{K d}\right)\right) ; Y=A \max /\left(1+10^{\wedge}(X-\log E C 50)\right)+A 0
$$

where $E C_{50}=$ Half maximal effective concentration $(\mu \mathrm{M}) ; K_{\mathrm{i}}=$ Inhibitor dissociation constant $(\mu \mathrm{M}), K_{\mathrm{d}}=$ fluorescein-labeled inhibitor.

1,2-di-acetamido-4-O-( $\beta$-galactopyranosyl)-1,2-dideoxy- $\beta$-D-glucopyranose ${ }^{4}$ was used as a positive control $\left(K_{\mathrm{d}} 36 \mathrm{nM}\right)$.

\section{General methods (Organic synthesis)}

All reactions with air- and moisture sensitive compounds were conducted in an atmosphere of nitrogen or argon. Dichloromethane, toluene, acetonitrile and THF were dried over aluminum oxide via an MBraun SPS-800 solvent purification system, other dry solvents were dried over molecular sieves. Evaporation of solvents was done under reduced pressure at $45^{\circ} \mathrm{C}$. Flash column chromatography was carried out with Merck silica gel $(5-40 \mu \mathrm{m})$ or Merck silica gel (230-400 mesh). TLC analysis was carried out on silica gel on aluminum foil (Merck Kieselgel $60 \mathrm{~F} 254$ ). TLC plates were visualized by using ceric sulfate/ammonium molybdate in $10 \% \mathrm{H}_{2} \mathrm{SO}_{4}$ and successive heating to dryness.

\section{Apparatus}

${ }^{1} \mathrm{H}$ and ${ }^{13} \mathrm{C}$ NMR were recorded using a Varian Mercury 400 spectrometer at either $400 \mathrm{MHz}$ or $100 \mathrm{MHz}$ respectively, or a Bruker 950 $\mathrm{MHz}$ at $950 \mathrm{MHz}$ or $239 \mathrm{mHz}$ respectively. Chemical shifts $(\delta)$ are given in ppm relative to the residual solvent signals $\left(\mathrm{CDCl}_{3}(\delta 7.26\right.$ ppm for proton and $77.16 \mathrm{ppm}$ for carbon resonances), $\mathrm{CD}_{3} \mathrm{OD}$ ( $\delta 3.31 \mathrm{ppm}$ for proton and 49.00 ppm for carbon resonances)) Assignment of NMR spectra is based on gCOSY, gHMQC and DEPT-135 experiments. Mass spectra were recorded on a Brücher maXis LCQ-TOF spectrometer with positive S2 electronspray ionization or on a Bruker Daltonics mass spectrometer for MALDI-TOF MS by using a 1,8-dihydroxyanthron (dithranol) matrix. UV irradiation of reactions was carried out using a Vilber Lourmat VL-6.LC UV lamp. Melting points were measured on a Büchi B-450, and are not corrected. Optical rotations were measured on an ADP440+ polarimeter and reported in units of deg $\mathrm{cm}^{2} \cdot \mathrm{g}^{-1}$. Concentrations are given in $\mathrm{g} / 100 \mathrm{~mL}$. 


\section{Synthesis of Group A building blocks}

\section{Synthesis of galacto azide A1}

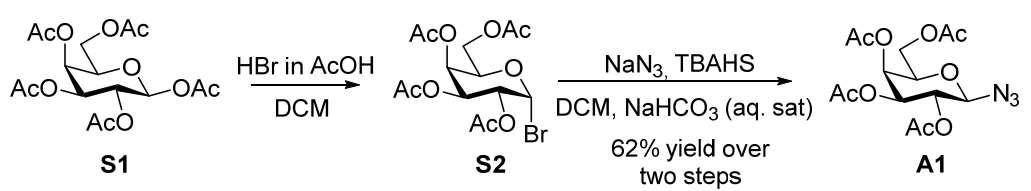

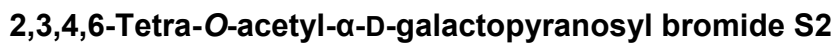

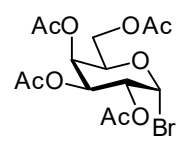

$\mathrm{HBr}$ in $\mathrm{AcOH}(33 \% \mathrm{w} / \mathrm{v}, 30 \mathrm{~mL}$ ) was added to a solution of $\beta$-D-galactose pentaacetate $\mathbf{S 1}$ (5.05 g; $12.9 \mathrm{mmol} ; 1$ equiv.) dissolved in dry dichloromethane $(40 \mathrm{~mL})$ under a $\mathrm{N}_{2}$ atm. After 1.5 hours, the reaction had reached completion, and was diluted with $\mathrm{H}_{2} \mathrm{O}$ and dichloromethane. The aqueous phase was extracted with dichloromethane, and the combined organic phases were washed with $\mathrm{H}_{2} \mathrm{O}$, then sat. $\mathrm{NaHCO}_{3}$ (aq.) and brine. The organic phase was dried over magnesium sulfate, filtered and evaporated to yield the crude product $\mathbf{S 2}$ as yellow oil. $R_{\mathrm{f}} 0.33$ (4:1 pentane/ethyl acetate). ${ }^{1} \mathrm{H}$ NMR $\left(400 \mathrm{MHz}, \mathrm{CDCl}_{3}\right) \delta_{\mathrm{H}} 6.69(\mathrm{~d}, \mathrm{~J}=3.9 \mathrm{~Hz}, 1 \mathrm{H}, \mathrm{H} 1), 5.51(\mathrm{~d}, \mathrm{~J}=$ $2.8 \mathrm{~Hz}, 1 \mathrm{H}, \mathrm{H} 4), 5.39$ (dd, J = 10.6, $2.8 \mathrm{~Hz}, 1 \mathrm{H}, \mathrm{H} 3$ ), 5.04 (dd, J = 10.6, 3.9 Hz, 1H, H2), 4.47 (t, J = 6.5 Hz, 1H, H5), 4.17 (dd, J = 11.4, $6.5 \mathrm{~Hz}, 1 \mathrm{H}, \mathrm{H} 6 \mathrm{a}), 4.10$ (dd, J = 11.4, $6.5 \mathrm{~Hz}, 1 \mathrm{H}, \mathrm{H} 6 \mathrm{~b}), 2.14\left(\mathrm{~s}, 3 \mathrm{H}, \mathrm{COCH}_{3}\right), 2.10\left(\mathrm{~s}, 3 \mathrm{H}, \mathrm{COCH}_{3}\right), 2.05\left(\mathrm{~s}, 3 \mathrm{H}, \mathrm{COCH}_{3}\right), 2.00(\mathrm{~s}, 3 \mathrm{H}$, $\left.\mathrm{COCH}_{3}\right) \cdot{ }^{13} \mathrm{C} \mathrm{NMR}\left(101 \mathrm{MHz}, \mathrm{CDCl}_{3}\right) \delta_{\mathrm{C}} 170.4\left(\mathrm{COCH}_{3}\right), 170.2\left(\mathrm{COCH}_{3}\right), 170.0\left(\mathrm{COCH}_{3}\right), 169.9\left(\mathrm{COCH}_{3}\right), 88.2(\mathrm{C} 1), 71.2(\mathrm{C} 5), 68.1$ (C3), 67.9 (C2), 67.1 (C4), 60.9 (C6), $20.9\left(\mathrm{COCH}_{3}\right), 20.8\left(\mathrm{COCH}_{3}\right), 20.7\left(\mathrm{COCH}_{3}\right), 20.7\left(\mathrm{COCH}_{3}\right)$. HRMS (ES): Calcd. for $\mathrm{C}_{14} \mathrm{H}_{19}{ }^{79} \mathrm{BrO}_{9} \mathrm{NH}_{4}$ : 428.0556; found 428.0558. Data are in accordance with published literature. ${ }^{5}$

\section{2,3,4,6-Tetra-O-acetyl- $\beta$-D-galactopyranosyl azide A1}

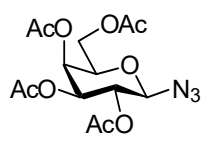

Bromide 12 (0.363 g; 0.882 mmol; 1 equiv.), $\mathrm{NaN}_{3}$ (0.287 g; $4.41 \mathrm{mmol} ; 5$ equiv.) and TBAHS (0.299 g; 0.882 mmol; 1 equiv.) were dissolved in dichloromethane $(6 \mathrm{~mL})$. Sat. $\mathrm{NaHCO}_{3}(\mathrm{aq})(6 \mathrm{~mL})$ was added and the solution was stirred vigorously at rt. After one hour the reaction had reached completion and was diluted with $\mathrm{H}_{2} \mathrm{O}$. The aqueous phase was extracted with ethyl acetate, and the combined organic phases were washed with sat. $\mathrm{NaHCO}_{3}(\mathrm{aq})$ then $\mathrm{H}_{2} \mathrm{O}$ and brine. The organic phase was dried over magnesium sulfate, filtered and evaporated. The product 9 was purified by column chromatography (pentane/ethyl acetate 2:1) and isolated as a white solid in $62 \%$ yield in two steps $\left(0.203 \mathrm{~g} ; 0.543 \mathrm{mmol}\right.$ ). $R_{\mathrm{f}} 0.28$ (pentane/ethyl acetate 2:1). $\mathrm{M}_{\mathrm{p}}$ (uncorr) 96.0-96.5 ${ }^{\circ} \mathrm{C}$. (Lit.: $92-94{ }^{\circ} \mathrm{C}$ ) ${ }^{6}{ }^{1} \mathrm{H}$ NMR $\left(400 \mathrm{MHz}, \mathrm{CDCl}_{3}\right) \delta_{\mathrm{H}} 5.41(\mathrm{~d}, \mathrm{~J}=2.9 \mathrm{~Hz}, 1 \mathrm{H}, \mathrm{H} 4), 5.15(\mathrm{dd}, \mathrm{J}=10.4,8.7 \mathrm{~Hz}, 1 \mathrm{H}, \mathrm{H} 2), 5.03(\mathrm{dd}, \mathrm{J}=10.4,2.9 \mathrm{~Hz}, 1 \mathrm{H}, \mathrm{H} 3), 4.59(\mathrm{~d}, \mathrm{~J}=$ $8.7 \mathrm{~Hz}, 1 \mathrm{H}, \mathrm{H} 1), 4.21-4.10(\mathrm{~m}, 2 \mathrm{H}, \mathrm{H} 6), 4.01(\mathrm{t}, \mathrm{J}=6.2 \mathrm{~Hz}, 1 \mathrm{H}, \mathrm{H} 5), 2.16\left(\mathrm{~s}, 3 \mathrm{H}, \mathrm{COCH}_{3}\right), 2.09\left(\mathrm{~s}, 3 \mathrm{H}, \mathrm{COCH}_{3}\right), 2.06(\mathrm{~s}, 3 \mathrm{H}, \mathrm{COCH})$, $1.98\left(\mathrm{~s}, 3 \mathrm{H}, \mathrm{COCH}_{3}\right) \cdot{ }^{13} \mathrm{C} \mathrm{NMR}\left(101 \mathrm{MHz}, \mathrm{CDCl}_{3}\right) \delta_{\mathrm{C}} 170.5,170.2,170.1,169.5\left(\mathrm{COCH}_{3}\right), 88.4$ (C1), 73.0 (C2), 70.9 (C5), 68.2 (C3), 67.0 (C4), 61.4 (C6), $20.8\left(\mathrm{COCH}_{3}\right), 20.6\left(\mathrm{COCH}_{3}\right)$. HRMS (ES): Calcd. for $\mathrm{C}_{14} \mathrm{H}_{19} \mathrm{~N}_{3} \mathrm{O}_{9} \mathrm{Na:}$ 396.1019; found: 396.1014. Data are in accordance with published literature. ${ }^{6}$

\section{Synthesis of galacto thiol A2}

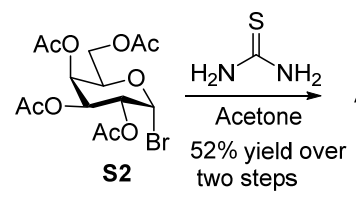

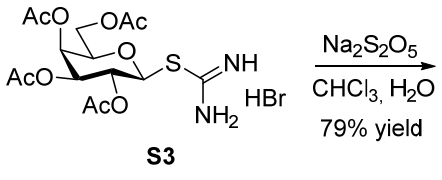

S3

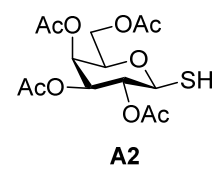




\section{2,3,4,6-Tetra-O-acetyl- $\beta$-D-galactopyranosyl-1-isothiouronium bromide S3}

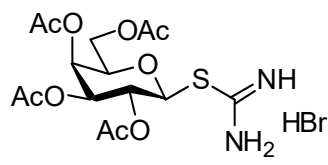

The bromide S2 (5.451 g; $0.0133 \mathrm{~mol} ; 1$ equiv.) and thiourea (1.514 g; $0.0199 \mathrm{~mol} ; 1.5$ equiv.) were dissolved in dry acetone (35 mL) under a $\mathrm{N}_{2}$ atm. The solution was heated to reflux, and after 2 hours a white precipitate formed. The precipitate was filtered of, and the filtrate was heated to reflux again. This process was repeated until formation of precipitate no longer occurred. The combined precipitates were recrystallized from isopropanol, and the final product $\mathbf{S} 3$ was obtained as white crystals in $52 \%$ yield over two steps (2.730 g; $6.72 \mathrm{mmol}$ ). $\mathrm{M}_{\mathrm{p}}$ (uncorr) 106.9-161.6 ${ }^{\circ} \mathrm{C}$. (Lit.: $\left.169.5^{\circ} \mathrm{C}^{7}\right){ }^{1} \mathrm{H}$ NMR (400 MHz, DMSO-d $\left.)\right) \delta_{\mathrm{H}} 9.33$ (br s, $\left.1 \mathrm{H}, \mathrm{NH}\right), 9.10$ (br s, $\left.1 \mathrm{H}, \mathrm{NH}_{2}\right), 5.67(\mathrm{~d}, \mathrm{~J}=9.9 \mathrm{~Hz}, 1 \mathrm{H}, \mathrm{H} 1), 5.39$ (d, J = 3.3 Hz, 1H, H4), 5.24 (dd, J = 9.9, 3.3 Hz, 1H, H3), $5.11(\mathrm{t}, \mathrm{J}=9.9 \mathrm{~Hz}, 1 \mathrm{H}, \mathrm{H} 2), 4.43$ $(\mathrm{t}, \mathrm{J}=6.1 \mathrm{~Hz}, 1 \mathrm{H}, \mathrm{H} 5), 4.16-4.00(\mathrm{~m}, 2 \mathrm{H}, \mathrm{H} 6), 2.14\left(\mathrm{~s}, 3 \mathrm{H}, \mathrm{COCH}_{3}\right), 2.09\left(\mathrm{~s}, 3 \mathrm{H}, \mathrm{COCH}_{3}\right), 2.01\left(\mathrm{~s}, 3 \mathrm{H}, \mathrm{COCH}_{3}\right), 1.95(\mathrm{~s}, 3 \mathrm{H}, \mathrm{COCH})$. ${ }^{13} \mathrm{C}$ NMR (101 MHz, DMSO-d6) $\delta_{\mathrm{C}}$ 169.9, 169.7, 169.4, $169.3\left(\mathrm{COCH}_{3}\right), 166.3(\mathrm{C}=\mathrm{N}), 80.2$ (C1), 74.4 (C5), 70.4 (C3), 67.1 (C4), 66.3 (C2), 61.3 (C6), 25.5, 20.6, 20.4, $20.3\left(\mathrm{COCH}_{3}\right)$. HRMS (ES): Calcd. for $\mathrm{C}_{15} \mathrm{H}_{22} \mathrm{~N}_{2} \mathrm{O}_{9} \mathrm{SH}$ : 407.1124; found 407.1124. Data are in accordance with published literature. ${ }^{5}$

\section{Thio- $\beta$-D-galactose A2}

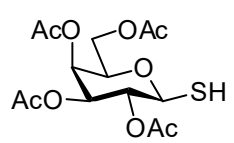

Sodium bisulfite $\left(0.231 \mathrm{~g} ; 2.22 \mathrm{mmol} ; 4.8\right.$ equiv.) was added to a solution of $\mathbf{S} 3$ ( $0.119 \mathrm{~g} ; 0.463 \mathrm{mmol} ; 1$ equiv.) dissolved in $\mathrm{H}_{2} \mathrm{O}(5$ $\mathrm{mL}$ ) and chloroform (10 mL) under a $\mathrm{N}_{2}$ atm. The mixture was heated to reflux for 6 hours until the reaction had reached completion. The reaction was then cooled to rt and diluted with $\mathrm{H}_{2} \mathrm{O}$. The aqueous phase was extracted with dichloromethane, and the combined organic phases were dried over magnesium sulfate, filtered and evaporated. The product $\mathbf{A} 2$ was obtained as a clear oil in $79 \%$ yield (0.085 g; $0.232 \mathrm{mmol}$ ). $R_{\mathrm{f}} 0.56$ (pentane/ethyl acetate 1:1). ${ }^{1} \mathrm{H}$ NMR $\left(400 \mathrm{MHz}, \mathrm{CDCl}_{3}\right) \delta_{\mathrm{H}} 5.40$ (dd, J = 3.4, $\left.1.1 \mathrm{~Hz}, 1 \mathrm{H}, \mathrm{H} 4\right), 5.15(\mathrm{t}, \mathrm{J}$ $=9.9 \mathrm{~Hz}, 1 \mathrm{H}, \mathrm{H} 2), 4.99(\mathrm{dd}, \mathrm{J}=9.9,3.4 \mathrm{~Hz}, 1 \mathrm{H}, \mathrm{H} 3), 4.51$ (t, J = 9.9 Hz, 1H, H1), 4.09 (d, J = 7.0 Hz, 2H, H6), $3.92(\mathrm{t}, J=7.0 \mathrm{~Hz}, 1 \mathrm{H}$, $\mathrm{H} 5), 2.35(\mathrm{~d}, \mathrm{~J}=9.9 \mathrm{~Hz}, 1 \mathrm{H}, \mathrm{SH}), 2.13\left(\mathrm{~s}, 3 \mathrm{H}, \mathrm{COCH}_{3}\right), 2.06\left(\mathrm{~s}, 3 \mathrm{H}, \mathrm{COCH}_{3}\right), 2.02\left(\mathrm{~s}, 3 \mathrm{H}, \mathrm{COCH}_{3}\right), 1.95\left(\mathrm{~s}, 3 \mathrm{H}, \mathrm{COCH}_{3}\right) .{ }^{13} \mathrm{C} \mathrm{NMR}$ $\left(101 \mathrm{MHz}, \mathrm{CDCl}_{3}\right) \delta_{\mathrm{C}} 170.4\left(\mathrm{COCH}_{3}\right), 170.2\left(\mathrm{COCH}_{3}\right), 170.0\left(\mathrm{COCH}_{3}\right), 169.9\left(\mathrm{COCH}_{3}\right), 79.2(\mathrm{C} 1), 75.0(\mathrm{C} 5), 71.6(\mathrm{C} 3), 70.9(\mathrm{C} 2), 67.3$ (C4), $61.5(\mathrm{C} 6), 20.9\left(\mathrm{COCH}_{3}\right), 20.8\left(\mathrm{COCH}_{3}\right), 20.7\left(\mathrm{COCH}_{3}\right), 20.6\left(\mathrm{COCH}_{3}\right)$. HRMS (ES): Calcd. for $\mathrm{C}_{14} \mathrm{H}_{20} \mathrm{O}_{9} \mathrm{SNH}_{4}: 382.1172$; found 382.1170. Data are in accordance with published literature. ${ }^{5}$

\section{Synthesis of manno azide A3}
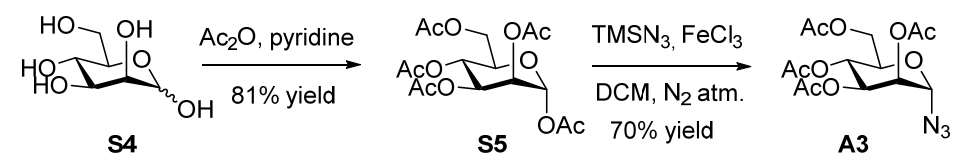

\section{1,2,3,4,6-Penta-O-acetyl- $\alpha$-D-mannopyranose S5}

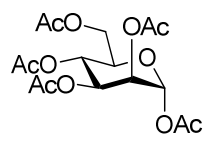

Dry pyridine (12 mL) was added to a solution of D-(+)-mannose $\mathbf{S 4}\left(5.00 \mathrm{~g} ; 27.8 \mathrm{mmol}\right.$; 1 equiv.) in acetic acid (12 mL) under a $\mathrm{N}_{2}$ atm. After 4 hours the reaction had reached completion and was quenched by dropwise addition of $\mathrm{H}_{2} \mathrm{O}$. After 20 min the mixture was diluted with ethyl acetate and the organic phase was washed with $1 \mathrm{M} \mathrm{HCl}(\mathrm{aq}), \mathrm{H}_{2} \mathrm{O}$, sat $\mathrm{NaHCO}_{3}$ and brine sequentially. The organic phase was then dried over magnesium sulfate, filtered and evaporated. The crude product $\mathbf{S} 5$ was obtained as clear oil in $81 \%$ yield $(8.78 \mathrm{~g}$; $22.5 \mathrm{mmol}) R_{\mathrm{f}} 0,46$ (pentane/ethyl acetate 1:1). ${ }^{1} \mathrm{H} \mathrm{NMR}\left(400 \mathrm{MHz}, \mathrm{CDCl}_{3}\right) \delta_{\mathrm{H}} 6.04(\mathrm{~d}, \mathrm{~J}=1.9 \mathrm{~Hz}, 1 \mathrm{H}, \mathrm{H} 1), 5.37-5.26(\mathrm{~m}, 2 \mathrm{H}, \mathrm{H} 3$ and 
$\mathrm{H} 4), 5.22(\mathrm{t}, \mathrm{J}=1.9 \mathrm{~Hz}, 1 \mathrm{H}, \mathrm{H} 2), 4.24(\mathrm{dd}, \mathrm{J}=12.3,4.8 \mathrm{~Hz}, 1 \mathrm{H}, \mathrm{H} 6 \mathrm{a}), 4.13-3.95(\mathrm{~m}, 2 \mathrm{H}, \mathrm{H} 5$ and H6b), $2.14(\mathrm{~s}, 3 \mathrm{H}, \mathrm{COCH}$ ), 2.13 (s, $\left.3 \mathrm{H}, \mathrm{COCH}_{3}\right), 2.05\left(\mathrm{~s}, 3 \mathrm{H}, \mathrm{COCH}_{3}\right), 2.01\left(\mathrm{~s}, 3 \mathrm{H}, \mathrm{COCH}_{3}\right), 1.96\left(\mathrm{~s}, 3 \mathrm{H}, \mathrm{COCH}_{3}\right) \cdot{ }^{13} \mathrm{C} \mathrm{NMR}\left(101 \mathrm{MHz}, \mathrm{CDCl}_{3}\right) \delta_{\mathrm{C}} 170.7\left(\mathrm{COCH}_{3}\right), 170.0$ $\left(\mathrm{COCH}_{3}\right), 169.8\left(\mathrm{COCH}_{3}\right), 169.6\left(\mathrm{COCH}_{3}\right), 168.1\left(\mathrm{COCH}_{3}\right), 90.6\left(\mathrm{C} 1\left(\mathrm{~J}_{\mathrm{C} 1, \mathrm{H} 1}=177.6 \mathrm{~Hz}\right.\right.$ (characteristic for $\alpha$-anomer $\left.\left.{ }^{8}\right)\right), 70.6(\mathrm{C} 5), 68.8$ (C3/C4), 68.3 (C2), 65.5 (C3/C4), 62.1 (C6), $20.9\left(\mathrm{COCH}_{3}\right), 20.8\left(\mathrm{COCH}_{3}\right), 20.7\left(\mathrm{COCH}_{3}\right), 20.7\left(\mathrm{COCH}_{3}\right), 20.7(\mathrm{COCH}) . \mathrm{HRMS}(\mathrm{ES})$ : Calcd. for $\mathrm{C}_{16} \mathrm{H}_{22} \mathrm{O}_{11} \mathrm{NH}_{4}: 408.1506$; found 408.1505 . Data are in accordance with published literature. ${ }^{9}$

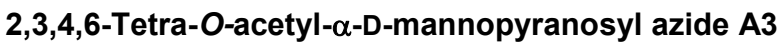

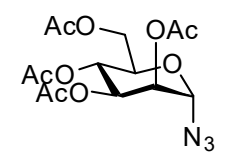

$\operatorname{TMSN}_{3}\left(0.25 \mathrm{~mL} ; 1.92 \mathrm{mmol} ; 1.5\right.$ equiv.) and $\mathrm{FeCl}_{3}(0.0206 \mathrm{~g} ; 0.129 \mathrm{mmol} ; 0.1$ equiv.) were added to a solution of peracetylated mannosan S5 (0.502 g; 1.28 mmol; 1 equiv.) dissolved in dry dichloromethane $(5 \mathrm{~mL})$ under a $\mathrm{N}_{2}$ atm. The reaction was stirred at rt overnight. When the reaction had run to completion it was diluted with dichloromethane, and the organic phase was washed with $\mathrm{H}_{2} \mathrm{O}$, dried over magnesium sulfate, filtered and evaporated. The product $\mathbf{A} \mathbf{3}$ was purified by column chromatography (pentane/ethyl acetate $2: 1)$ and obtained as clear oil in $70 \%$ yield $(0.334 \mathrm{~g} ; 0.895 \mathrm{mmol}) . R_{\mathrm{f}} 0.54$ (pentane/ethyl acetate 1:1). $[\alpha]_{\mathrm{D}}^{295 \mathrm{~K}}+102.6(\mathrm{c} 1, \mathrm{CHCl})(\mathrm{Lit} .:$ $\left.+102\left(\mathrm{c} 1, \mathrm{CHCl}_{3}\right)\right) .{ }^{6}{ }^{1} \mathrm{H}$ NMR $\left(400 \mathrm{MHz}, \mathrm{CDCl}_{3}\right) \delta_{\mathrm{H}} 5.32(\mathrm{~d}, \mathrm{~J}=1.8 \mathrm{~Hz}, 1 \mathrm{H}, \mathrm{H} 1), 5.23-5.10(\mathrm{~m}, 2 \mathrm{H}, \mathrm{H} 3$ and H4), $5.06(\mathrm{dd}, \mathrm{J}=2.9,1.8$ $\mathrm{Hz}, 1 \mathrm{H}, \mathrm{H} 2$ ), 4.21 (dd, $\mathrm{J}=12.7,5.8 \mathrm{~Hz}, 1 \mathrm{H}, \mathrm{H} 6 \mathrm{a}), 4.10-4.00$ (m, 2H, H5 and H6b), 2.07 (s, 3H, COCH $\mathrm{CO}_{3}, 2.01\left(\mathrm{~s}, 3 \mathrm{H}, \mathrm{COCH}_{3}\right), 1.96$ $\left(\mathrm{s}, 3 \mathrm{H}, \mathrm{COCH}_{3}\right), 1.89\left(\mathrm{~s}, 3 \mathrm{H}, \mathrm{COCH}_{3}\right) \cdot{ }^{13} \mathrm{C} \mathrm{NMR}\left(101 \mathrm{MHz}, \mathrm{CDCl}_{3}\right) \delta_{\mathrm{C}} 170.4\left(\mathrm{COCH}_{3}\right), 169.7\left(\mathrm{COCH}_{3}\right), 169.6\left(\mathrm{COCH}_{3}\right), 169.5\left(\mathrm{COCH}_{3}\right)$, $87.3\left(\mathrm{C} 1\left(\mathrm{~J}_{\mathrm{C} 1, \mathrm{H} 1}=170.7 \mathrm{~Hz}\right.\right.$ (characteristic of $\alpha$-anomer)), 70.5 (C5), 69.0 (C2), 68.1 (C3/C4), $65.4(\mathrm{C} 3 / \mathrm{C} 4), 62.0(\mathrm{C} 6), 20.6(\mathrm{COCH})$, $20.5\left(\mathrm{COCH}_{3}\right), 20.5\left(\mathrm{COCH}_{3}\right), 20.4\left(\mathrm{COCH}_{3}\right)$. HRMS (ES): Calcd. for $\mathrm{C}_{14} \mathrm{H}_{19} \mathrm{~N}_{3} \mathrm{O}_{9} \mathrm{NH}_{4}: 391.1465$ found 391.1464. Data are in accordance with published literature. ${ }^{9}$

\section{Synthesis of manno thiol A4}

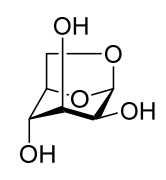

S6

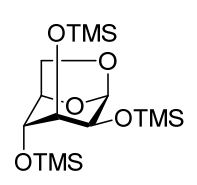

S7

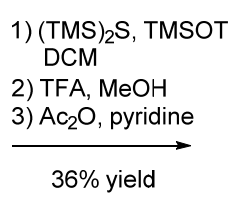

$36 \%$ yield

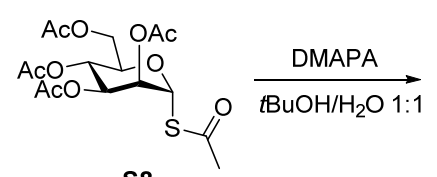

S8

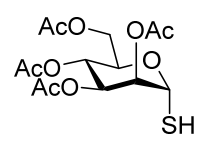

A4

\section{1,6-anhydro-2,3,4-tris-O-trimethylsilyl- $\beta$-D-mannopyranose S7}

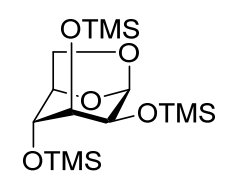

Mannosan S6 (0.123 g; $0.761 \mathrm{mmol} ; 1$ equiv.) and triethyl amine ( $0.53 \mathrm{~mL} ; 3.81 \mathrm{mmol} ; 5$ equiv.) were dissolved in dry dichloromethane $(2.5 \mathrm{~mL})$ under a $\mathrm{N}_{2}$ atm. The solution was cooled to $0{ }^{\circ} \mathrm{C}$ before $\mathrm{TMSCl}(0.44 \mathrm{~mL} ; 3.50 \mathrm{mmol} ; 4$ equiv.) was added dropwise. The reaction was then allowed to warm to $\mathrm{rt}$ and left overnight. The solvent was evaporated and the residue was diluted with pentane and filtered. The solid was washed with pentane and the filtrate was evaporated. The product $\mathbf{S} 7$ was purified by column chromatography (pentane/ethyl acetate 3:0.1) and isolated as light yellow oil in $86 \%$ yield $\left(0.247 \mathrm{~g} ; 0.651 \mathrm{mmol}\right.$ ). $R_{\mathrm{f}} 0.64$ (pentane/ethyl acetate $\left.3: 0.2\right)$. $[\alpha]_{D}{ }^{295 K}-68.0\left(c\right.$ 1, $\left.\mathrm{CHCl}_{3}\right)\left(\mathrm{Lit.}:-70.5\left(c \mathrm{c}, \mathrm{CHCl}_{3}\right)\right) .{ }^{10}{ }^{1} \mathrm{H} \mathrm{NMR}\left(400 \mathrm{MHz}, \mathrm{CDCl}_{3}\right) \delta_{\mathrm{H}} 5.23(\mathrm{~s}, 1 \mathrm{H}, \mathrm{H} 1), 4.33-4.24(\mathrm{~m}, 2 \mathrm{H}, \mathrm{H} 3 / \mathrm{H} 4 / \mathrm{H} 5$ and $\mathrm{H} 6 \mathrm{a}), 3.79-3.62(\mathrm{~m}, 4 \mathrm{H}, \mathrm{H} 3 / \mathrm{H} 4 / \mathrm{H} 5$ and $\mathrm{H} 2, \mathrm{H} 6 \mathrm{~b}), 0.18\left(\mathrm{~s}, 9 \mathrm{H}, 3 \mathrm{CH}_{3}\right), 0.16\left(\mathrm{~s}, 9 \mathrm{H}, 3 \mathrm{CH}_{3}\right), 0.14\left(\mathrm{~s}, 9 \mathrm{H}, 3 \mathrm{CH}_{3}\right) .{ }^{13} \mathrm{C} \mathrm{NMR}(101 \mathrm{MHz}$, $\left.\mathrm{CDCl}_{3}\right) \delta_{\mathrm{C}} 102.4(\mathrm{C} 1), 76.5,74.7,74.2,68.4$ (C2, C3 C4 and C5), $64.8(\mathrm{C} 6), 0.5\left(\mathrm{CH}_{3}\right), 0.4\left(\mathrm{CH}_{3}\right), 0.2\left(\mathrm{CH}_{3}\right)$. HRMS (ES): Calcd. for $\mathrm{C}_{15} \mathrm{H}_{34} \mathrm{O}_{5} \mathrm{Si}_{3} \mathrm{H}: 379.1792$ found 379.1790 . Data are in accordance with published literature. ${ }^{10}$ 


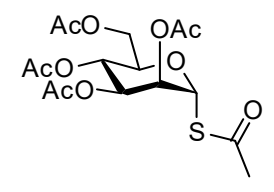

TMSOTf (0.03 mL; $0.174 \mathrm{mmol} ; 0.5$ equiv.) was added dropwise to a solution of per-TMS protected mannosan S7 (0.132 g; 0.347 mmol; 1 equiv.) and (TMS $)_{2} \mathrm{~S}\left(0.14 \mathrm{~mL} ; 0.695 \mathrm{mmol} ; 2\right.$ equiv.) dissolved in dry dichloromethane $(6 \mathrm{~mL})$ under a $\mathrm{N}_{2}$ atm. The mixture was heated to reflux and left overnight. When the reaction had reached completion methanol ( $3.5 \mathrm{~mL})$ and TFA (35 $\square \mathrm{L})$ was added, and after $15 \mathrm{~min}$ the solvents were evaporated and co-evaporated with toluene. The residue was dissolved in dry pyridine (2 $\mathrm{mL})$ and acetic anhydride $(2 \mathrm{~mL})$ under a $\mathrm{N}_{2}$ atm. The acetylation reaction was left overnight. When the reaction had reached completion, it was quenched by dropwise addition of $\mathrm{H}_{2} \mathrm{O}$. After 20 minutes the mixture was diluted with ethyl acetate and the organic phase was washed with $1 \mathrm{M} \mathrm{HCl}(\mathrm{aq}), \mathrm{H}_{2} \mathrm{O}$, sat $\mathrm{NaHCO}_{3}$ and brine sequentially. The organic phase was then dried over magnesium sulfate, filtered and evaporated. The product $\mathbf{S 8}$ was purified by column chromatography (dichloromethane/diethyl ether 4:0.1) and obtained as clear oil in $36 \%$ yield $(0.050 \mathrm{~g} ; 0.123 \mathrm{mmol}) . R_{\mathrm{f}} 0.47\left(1: 1\right.$ pentane/ethyl acetate). $[\alpha]_{\mathrm{D}}{ }^{295 \mathrm{~K}}+58.8\left(\mathrm{c} 1, \mathrm{CHCl}_{3}\right)$. [Lit.: $\left.+60.6(\mathrm{c} 1.11, \mathrm{CHCl})^{11}\right] .{ }^{1} \mathrm{H}$ $\operatorname{NMR}\left(400 \mathrm{MHz}, \mathrm{CDCl}_{3}\right) \delta_{\mathrm{H}} 5.94(\mathrm{~d}, \mathrm{~J}=1.7 \mathrm{~Hz}, 1 \mathrm{H}, \mathrm{H} 1), 5.40-5.25(\mathrm{~m}, 2 \mathrm{H}, \mathrm{H} 2$ and H4), 5.09 (dd, J = 10.1, 3.3 Hz, 1H, H3), $4.27(\mathrm{dd}$, $J=12.4,4.8 \mathrm{~Hz}, 1 \mathrm{H}, \mathrm{H6a}$ ), 4.06 (dd, $\mathrm{J}=12.4,2.4 \mathrm{~Hz}, 1 \mathrm{H}, \mathrm{H6b}$ ), 3.92 (ddd, J = 9.9, 4.7, $2.4 \mathrm{~Hz}, 1 \mathrm{H}, \mathrm{H} 5$ ), $2.42(\mathrm{~s}, 3 \mathrm{H}, \mathrm{SCOCH}$ ), 2.18 $\left(\mathrm{s}, 3 \mathrm{H}, \mathrm{COCH}_{3}\right), 2.08\left(\mathrm{~s}, 3 \mathrm{H}, \mathrm{COCH}_{3}\right), 2.04\left(\mathrm{~s}, 3 \mathrm{H}, \mathrm{COCH}_{3}\right), 1.99\left(\mathrm{~s}, 3 \mathrm{H}, \mathrm{COCH}_{3}\right) .{ }^{13} \mathrm{C} \mathrm{NMR}\left(101 \mathrm{MHz}, \mathrm{CDCl}_{3}\right) \delta_{\mathrm{C}} 190.5\left(\mathrm{SCOCH}_{3}\right)$, $170.7\left(\mathrm{COCH}_{3}\right), 170.0\left(\mathrm{COCH}_{3}\right), 169.9\left(\mathrm{COCH}_{3}\right), 169.6\left(\mathrm{COCH}_{3}\right), 80.3\left(\mathrm{C} 1, \mathrm{~J}_{\mathrm{C} 1, \mathrm{H} 1}=168.9 \mathrm{~Hz}\right.$ (characteristic of $\alpha$-anomer $\left.\left.{ }^{8}\right)\right), 72.5(\mathrm{C} 5)$, 71.1 (C2/C4), 70.0 (C3), 65.7 (C4/C2), $62.2(\mathrm{C} 6), 31.4\left(\mathrm{SCOCH}_{3}\right), 21.0\left(\mathrm{COCH}_{3}\right), 20.8\left(\mathrm{COCH}_{3}\right), 20.8\left(\mathrm{COCH}_{3}\right), 20.7\left(\mathrm{COCH}_{3}\right)$. HRMS (ES): Calcd. for $\mathrm{C}_{16} \mathrm{H}_{22} \mathrm{O}_{10} \mathrm{SNH}_{4}: 424.1277$ found 424.1274. Data are in accordance with published literature. ${ }^{12}$

\section{2,3,4,6-Tetra-O-acetyl- $\alpha-D-m a n n o s y l$ thiol A4}

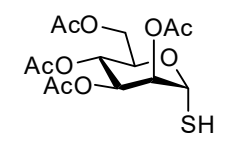

Dimethylaminopropyl amine (DMAPA) $(0.056 \mathrm{~mL} ; 0.444 \mathrm{mmol} ; 1.2$ equiv.) was added to a solution of thioacetate $\mathbf{S 8}(0.150 \mathrm{~g} ; 3.70$ mmol; 1 equiv.) dissolved in a 1:1 solution of degassed $t \mathrm{BuOH} / \mathrm{H}_{2} \mathrm{O}$ under a $\mathrm{N}_{2}$ atm. After one hour the reaction was diluted with dichloromethane and the organic phase was washed with $1 \mathrm{M} \mathrm{HCl}(\mathrm{aq})$ and brine, dried over magnesium sulfate, filtered and evaporated. The crude product $\mathbf{A} 4$ was used in the following reaction without further purification. $R_{\mathrm{f}} 0.28$ (pentane/ethyl acetate $\left.1: 1\right)$ ) $[\alpha]_{D}{ }^{295 K}-16.6$ (c 1, $\left.\mathrm{CHCl}_{3}\right)$. [Lit.: +78.6 (c 0.77, $\left.\mathrm{CHCl}_{3}\right)^{13}$ ]. ${ }^{1} \mathrm{H} \mathrm{NMR}\left(400 \mathrm{MHz}, \mathrm{CDCl}_{3}\right) \delta_{\mathrm{H}} 5.52(\mathrm{~d}, \mathrm{~J}=6.9 \mathrm{~Hz}, 1 \mathrm{H}, \mathrm{H} 1), 5.40-5.18(\mathrm{~m}, 3 \mathrm{H}, \mathrm{H} 2, \mathrm{H} 3 \mathrm{and}$ $\mathrm{H} 4), 4.36-4.29(\mathrm{~m}, 1 \mathrm{H}, \mathrm{H} 5), 4.26$ (dd, J = 12.2, $5.0 \mathrm{~Hz}, 1 \mathrm{H}, \mathrm{H6a}), 4.07$ (dd, J = 12.2, $2.0 \mathrm{~Hz}, 1 \mathrm{H}, \mathrm{H} 6 \mathrm{~b}), 2.28(\mathrm{~d}, \mathrm{~J}=6.9 \mathrm{~Hz}, 1 \mathrm{H}, \mathrm{SH})$,

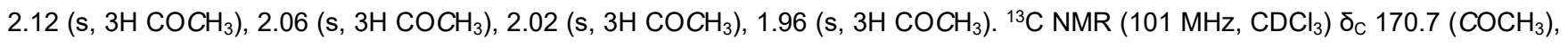
$\left.169.9\left(\mathrm{COCH}_{3}\right), 169.9\left(\mathrm{COCH}_{3}\right), 169.7\left(\mathrm{COCH}_{3}\right), 77.0\left(\mathrm{C} 1, J_{\mathrm{C} 1, \mathrm{H} 1}=170.0 \mathrm{~Hz} \text { (characteristic of } \alpha \text {-anomer }\right)^{8}\right), 71.9(\mathrm{C} 2, \mathrm{C} 3$ or C4) 69.6 (C5), 68.5 (C2, C3 or C4), 66.1 (C2, C3 or C4), $62.2(\mathrm{C} 6), 20.9\left(\mathrm{COCH}_{3}\right), 20.8\left(\mathrm{COCH}_{3}\right), 20.7\left(\mathrm{COCH}_{3}\right), 20.6(\mathrm{COCH})$. HRMS (ES): Calcd. for $\mathrm{C}_{14} \mathrm{H}_{20} \mathrm{O}_{9} \mathrm{SNH}_{4}$ : 382.1172 found 382.1173 . Data are in accordance with published literature. ${ }^{12}$

\section{Synthesis of GIcNAc azide A5}
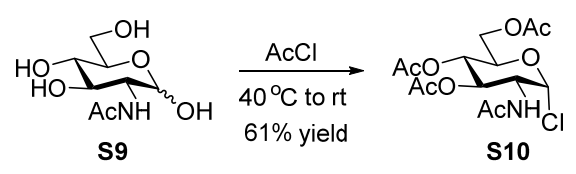
$\underset{\mathrm{NaN}_{3}, \text { TBAHS }}{\longrightarrow}$ $\mathrm{DCM}, \mathrm{NaHCO}_{3}$ $92 \%$ yield

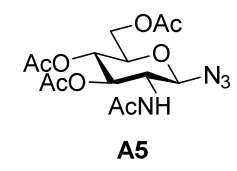

\section{2-Acetamido-3,4,6-tri-O-acetyl-2-deoxy- $\alpha$-D-glucopyranosyl chloride S10}




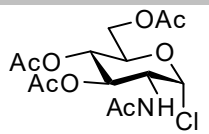

Acetyl chloride (15 mL) was added to GlcNAc S9 (5.0122 g; 22.7 mmol; 1 equiv.) under a $\mathrm{N}_{2}$ atm. The slurry was heated to $40{ }^{\circ} \mathrm{C}$ for one hour, until a color change from white to pink was observed. The reaction was stirred vigorously at rt overnight. The reaction mixture was then diluted with dichloromethane and poured into ice cold water. The organic phase was washed with sat. $\mathrm{NaHCO}_{3}(\mathrm{aq})$, dried over magnesium sulfate, filtered and evaporated. The product $\mathbf{S 1 0}$ was purified by column chromatography (pentane/ethyl acetate 1:1) and obtained as a light yellow solid in $61 \%$ yield $\left(5.09 \mathrm{~g} ; 13.9 \mathrm{mmol}\right.$ ). $R_{\mathrm{f}} 0.42$ (ethyl acetate/pentane $\left.2: 1\right) . \mathrm{M}_{\mathrm{p}}\left(\right.$ uncorr.) $124.0-125.1{ }^{\circ} \mathrm{C}$ (Lit.: $\left.124.0-126.0{ }^{\circ} \mathrm{C}^{14}\right)$. NMR $\left(400 \mathrm{MHz}, \mathrm{CDCl}_{3}\right) \delta_{\mathrm{H}} 6.17(\mathrm{~d}, \mathrm{~J}=3.6 \mathrm{~Hz}, 1 \mathrm{H}, \mathrm{H} 1), 5.91(\mathrm{~d}, \mathrm{~J}=8.6 \mathrm{~Hz}, 1 \mathrm{H}, \mathrm{NH}), 5.31(\mathrm{t}, \mathrm{J}=9.8 \mathrm{~Hz}, 1 \mathrm{H}$, $\mathrm{H} 3$ ), $5.20(\mathrm{t}, \mathrm{J}=9.8 \mathrm{~Hz}, 1 \mathrm{H}, \mathrm{H} 4), 4.52$ (ddd, $\mathrm{J}=9.8,8.6,3.6 \mathrm{~Hz}, 1 \mathrm{H}, \mathrm{H} 2), 4.37-4.19(\mathrm{~m}, 2 \mathrm{H}, \mathrm{H} 5$ and H6a), $4.11(\mathrm{~d}, \mathrm{~J}=10.7 \mathrm{~Hz}, 1 \mathrm{H}$, $\mathrm{H} 6 \mathrm{~b}), 2.09$ (s, 3H, $\left.\mathrm{COCH}_{3}\right), 2.03\left(\mathrm{~s}, 6 \mathrm{H}, \mathrm{COCH}_{3}\right.$, and $\left.\mathrm{NHCOCH}_{3}\right), 1.97\left(\mathrm{~s}, 3 \mathrm{H}, \mathrm{COCH}_{3}\right) .{ }^{13} \mathrm{C} \mathrm{NMR}\left(101 \mathrm{MHz}, \mathrm{CDCl}_{3}\right) \delta_{\mathrm{C}} 171.6\left(\mathrm{COCH}_{3}\right)$, $170.7\left(\mathrm{COCH}_{3}\right), 170.3\left(\mathrm{COCH}_{3}\right), 169.2\left(\mathrm{COCH}_{3}\right), 93.8(\mathrm{C} 1), 71.0(\mathrm{C} 5), 70.2(\mathrm{C} 3), 67.1(\mathrm{C} 4), 61.2(\mathrm{C} 6), 53.5(\mathrm{C} 2), 23.2(\mathrm{COCH}), 20.8$ $\left(\mathrm{COCH}_{3}\right), 20.7\left(\mathrm{COCH}_{3}\right)$. HRMS (ES): Calcd. for $\mathrm{C}_{14} \mathrm{H}_{20} \mathrm{NClO}_{8} \mathrm{H}$ : 366.0956; found 366.0957. Data are in accordance with published literature. ${ }^{15}$

\section{2-Acetamido-3,4,6-tri-O-acetyl-2-deoxy- $\beta$-D-glucopyranosyl azide A5}

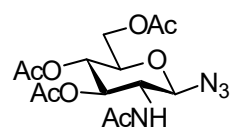

The GlcNAc chloride $\mathbf{S} 10$ (5.02 g; 0.13 .7 mmol; 1 equiv.), NaN $\mathrm{Na}_{3}(0.4 .46 \mathrm{~g} ; 68.6 \mathrm{mmol} ; 5$ equiv.) and TBASH (4.66 g; $13.7 \mathrm{mmol} ; 1$ equiv.) were dissolved in dichloromethane $(80 \mathrm{~mL})$. sat. $\mathrm{NaHCO}_{3}(\mathrm{aq})(80 \mathrm{~mL})$ was added and the solution was stirred vigorously at rt. After four hours the reaction had reached completion and was diluted with $\mathrm{H}_{2} \mathrm{O}$. The aqueous phase was extracted with dichloromethane, and the combined organic phases were washed with sat. $\mathrm{NaHCO}_{3}(\mathrm{aq})$. The organic phase was dried over magnesium sulfate, filtered and evaporated. The product $\mathbf{A} 5$ was purified by column chromatography (ethyl acetate/pentane 2:1) and isolated as a white solid in $92 \%$ yield $\left(0.4 .86 \mathrm{~g} ; 13.0 \mathrm{mmol}\right.$ ). $R_{\mathrm{f}} 0.33$ (ethyl acetate/pentane 3:1). $\mathrm{M}_{\mathrm{p}}$ (uncorr) 157.5-158.2 ${ }^{\circ} \mathrm{C}$. Lit.: $\left(161{ }^{\circ} \mathrm{C}^{16}\right)$. $[\alpha]_{\mathrm{D}}^{295 \mathrm{~K}}-4.4(c 1$, $\mathrm{CHCl}_{3}$ ) (Lit.: -18.9 (c 0.55, $\left.\mathrm{CHCl}_{3}\right)$ ). ${ }^{16}{ }^{1} \mathrm{H}$ NMR $\left(400 \mathrm{MHz}, \mathrm{CDCl}_{3}\right) \delta_{\mathrm{H}} 5.76(\mathrm{~d}, \mathrm{~J}=9.3 \mathrm{~Hz}, 1 \mathrm{H}, \mathrm{NH}), 5.24(\mathrm{dd}, \mathrm{J}=10.4,9.3 \mathrm{~Hz}, 1 \mathrm{H}, \mathrm{H} 3)$, 5.09 (t, J = 9.3 Hz, 1H, H4), 4.76 (d, J = 9.3 Hz, 1H, H1), 4.27 (dd, J = 12.4, 4.7 Hz, 1H, H6a), 4.16 (dd, J = 12.4, 2.2 Hz, 1H, H6b), 3.91 (q, $J=9.3 \mathrm{~Hz}, 1 \mathrm{H}, \mathrm{H} 2$ ), 3.79 (ddd, J = 9.3, 4.7, 2.2 Hz, 1H, H5), 2.10 (s, 3H, COCH $), 2.04$ (s, 3H, COCH $), 2.03\left(\mathrm{~s}, 3 \mathrm{H}, \mathrm{COCH}_{3}\right.$ ), $1.98\left(\mathrm{~s}, 3 \mathrm{H}, \mathrm{COCH}_{3}\right) .{ }^{13} \mathrm{C} \mathrm{NMR}\left(101 \mathrm{MHz}, \mathrm{CDCl}_{3}\right) \delta_{\mathrm{C}} 171.1\left(\mathrm{COCH}_{3}\right), 170.8\left(\mathrm{COCH}_{3}\right), 170.6\left(\mathrm{COCH}_{3}\right), 169.4\left(\mathrm{COCH}_{3}\right), 88.5(\mathrm{C} 1), 74.1$ (C5), 72.2 (C3), 68.1 (C4), 62.0 (C6), 54.3 (C2), $23.4\left(\mathrm{COCH}_{3}\right), 20.9\left(\mathrm{COCH}_{3}\right), 20.8\left(\mathrm{COCH}_{3}\right), 20.7\left(\mathrm{COCH}_{3}\right)$. HRMS (ES): Calcd. for $\mathrm{C}_{14} \mathrm{H}_{20} \mathrm{~N}_{4} \mathrm{O}_{8} \mathrm{H}: 373.1359$; found. 373.1353. Data are in accordance with published literature. ${ }^{17}$

\section{Synthesis of GIcNAc thiol A6}

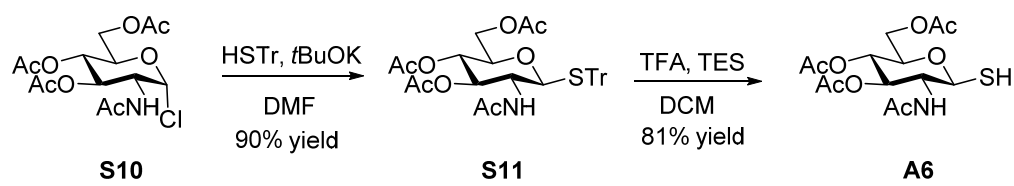

TriphenyImethyl 1-thio-2-acetamido-3,4,6-tri-O-acetyl-2-deoxy- $\beta$-D-glucopyranoside S11

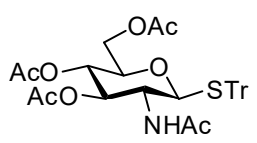

The GlcNAc chloride S10 (3.88 g; 10.6 mmol; 1 equiv.) was dissolved in dry DMF (12 mL) under a $\mathrm{N}_{2}$ atm. In a separate flask HSTr (3.52 g; 12.7 mmol; 1.2 equiv.) was dissolved in dry DMF (24 mL) before tBuOK (1.43 g; $12.7 \mathrm{mmol} ; 1.2$ equiv.) was slowly added 
under a $\mathrm{N}_{2}$ atm. The HSTr and tBuOK solution was stirred for 15 min before it was added dropwise to the chloride solution. When the reaction had reached completion it was diluted with ethyl acetate, and the organic phase was washed with $\mathrm{H}_{2} \mathrm{O}$ and brine, dried over magnesium sulfate, filtered and evaporated. The product $\mathbf{S 1 1}$ was purified by column chromatography (pentane/ethyl acetate 1:1) and isolated as white foam in $90 \%$ yield $(5.77 \mathrm{~g} ; 9.52 \mathrm{mmol}) . R_{\mathrm{f}} 0.57$ (dichloromethane/ethyl acetate 2:1). [ $\left.\alpha\right]_{\mathrm{D}}^{295 \mathrm{~K}}-88.8(\mathrm{C} 1, \mathrm{CHCl})[\mathrm{Lit}$ : -13.5 (c 1.02, $\left.\mathrm{CHCl}_{3}{ }^{18}\right)$ ]. ${ }^{1} \mathrm{H}$ NMR $\left(400 \mathrm{MHz} \mathrm{CDCl}_{3}\right) \delta_{\mathrm{H}} 7.50-7.41(\mathrm{~m}, 6 \mathrm{H}, \mathrm{ArH}), 7.31-7.17(\mathrm{~m}, 9 \mathrm{H}, \mathrm{ArH}), 4.97(\mathrm{t}, \mathrm{J}=10.2 \mathrm{~Hz}, 1 \mathrm{H}$, $\mathrm{H} 4), 4.90(\mathrm{~d}, \mathrm{~J}=9.9 \mathrm{~Hz}, 1 \mathrm{H}, \mathrm{NH}), 4.66(\mathrm{t}, \mathrm{J}=10.2 \mathrm{~Hz}, 1 \mathrm{H}, \mathrm{H} 3), 4.28(\mathrm{q}, \mathrm{J}=10.2 \mathrm{~Hz}, 1 \mathrm{H}, \mathrm{H} 2), 4.00(\mathrm{dd}, \mathrm{J}=12.2,5.2 \mathrm{~Hz}, 1 \mathrm{H}, \mathrm{H} 6 \mathrm{a}), 3.83$ (dd, $J=12.2,2.1 \mathrm{~Hz}, 1 \mathrm{H}, \mathrm{H} 6 \mathrm{~b}), 3.44$ (d, J = 10.2 Hz, 1H, H1), 2.93 (ddd, J = 10.2, 5.2, 2.1 Hz, 1H, H5), 1.95 (s, 3H, COCH ), 1.94 (s, $\left.3 \mathrm{H}, \mathrm{COCH}_{3}\right), 1.93\left(\mathrm{~s}, 3 \mathrm{H}, \mathrm{COCH}_{3}\right), 1.86\left(\mathrm{~s}, 3 \mathrm{H}, \mathrm{COCH}_{3}\right) .{ }^{13} \mathrm{C} \mathrm{NMR}\left(101 \mathrm{MHz}, \mathrm{CDCl}_{3}\right) \delta_{\mathrm{C}} 171.3\left(\mathrm{COCH}_{3}\right), 170.7\left(\mathrm{COCH}_{3}\right), 169.7$ $\left(\mathrm{COCH}_{3}\right), 169.2\left(\mathrm{COCH}_{3}\right), 144.5,130.0,127.8,127.0(\mathrm{ArC}), 84.6(\mathrm{C} 1), 75.6 .(\mathrm{C} 5), 74.5$ (C3), 68.8 (SCPh $), 68.2$ (C4), 62.3 (C6), 52.5 (C2), $23.4\left(\mathrm{COCH}_{3}\right), 20.8\left(\mathrm{COCH}_{3}\right), 20.7\left(\mathrm{COCH}_{3}\right), 20.6\left(\mathrm{COCH}_{3}\right)$. HRMS (ES): Calcd. for $\mathrm{C}_{33} \mathrm{H}_{35} \mathrm{NO}_{8} \mathrm{SNa}: 628.1981$; found. 628.1974. Data are in accordance with published literature. ${ }^{18}$

\section{2-Acetamido-3,4,6-tri-O-acetyl-2-deoxy- $\beta$-D-glucopyranosyl thiol A6}

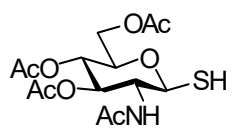

Triethylsilane ( $0.48 \mathrm{~mL} ; 5.88 \mathrm{mmol} ; 2.3$ equiv) and TFA (20 mL) was added to a solution of the trityl-protected thiol S11 (1.550 g; 2.56 mmol; 1 equiv.) dissolved in dry dichloromethane (30 mL) under a $\mathrm{N}_{2}$ atm. After 1 hour the reaction had reached completion and the solvents were evaporated and co-evaporated with toluene. The residue was then purified by column chromatography (ethyl acetate/pentane $3: 2$ ) and isolated as a white solid in $81 \%$ yield $\left(0.750 \mathrm{~g} ; 2.08 \mathrm{mmol}\right.$ ). $R_{\mathrm{f}} 0.33$ (ethyl acetate). $\mathrm{M}_{\mathrm{p}}$ (uncorr) $157.6-158.3^{\circ} \mathrm{C}$. Lit.: $\left(145-146{ }^{\circ} \mathrm{C}^{5}\right) .[\alpha]_{D}{ }^{295 \mathrm{~K}}-12.0\left(\mathrm{c} 1, \mathrm{CHCl}_{3}\right)$ [Lit.: $\left.-15.5\left(c \text { 1, } \mathrm{CHCl}_{3}\right)^{5}\right] .{ }^{1} \mathrm{H} \mathrm{NMR}\left(400 \mathrm{MHz}, \mathrm{CDCl}_{3}\right) \delta_{\mathrm{H}} 5.87(\mathrm{~d}, \mathrm{~J}=9.4 \mathrm{~Hz}, 1 \mathrm{H}, \mathrm{NH})$, $5.16-4.99(\mathrm{~m}, 2 \mathrm{H}, \mathrm{H} 3$ and H4), 4.59 (t, J = 9.4 Hz, 1H, H1), 4.22 (dd, J = 12.4, 4.8 Hz, 1H, H6a), 4.11 (d, J = 10.6 Hz, 2H, H2 and $\mathrm{H} 6 \mathrm{~b}), 3.73-3.60(\mathrm{~m}, 1 \mathrm{H}, \mathrm{H} 5), 2.55(\mathrm{~d}, \mathrm{~J}=9.4 \mathrm{~Hz}, 1 \mathrm{H}, \mathrm{SH}), 2.08\left(\mathrm{~s}, 3 \mathrm{H}, \mathrm{COCH}_{3}\right), 2.03\left(\mathrm{~s}, 3 \mathrm{H}, \mathrm{COCH}_{3}\right), 2.01\left(\mathrm{~s}, 3 \mathrm{H}, \mathrm{COCH}_{3}\right), 1.97(\mathrm{~s}$, $\left.3 \mathrm{H}, \mathrm{COCH}_{3}\right) \cdot{ }^{13} \mathrm{C} \mathrm{NMR}\left(101 \mathrm{MHz}, \mathrm{CDCl}_{3}\right) \delta_{\mathrm{C}} 171.3\left(\mathrm{COCH}_{3}\right), 170.9\left(\mathrm{COCH}_{3}\right), 170.6\left(\mathrm{COCH}_{3}\right), 169.4\left(\mathrm{COCH}_{3}\right), 80.4(\mathrm{C} 1), 76.3(\mathrm{C} 5)$, 73.6, 68.2 (C3 and C4), $62.3(\mathrm{C} 6), 56.9(\mathrm{C} 2), 23.4\left(\mathrm{NHCOCH}_{3}\right), 20.9\left(\mathrm{COCH}_{3}\right), 20.8\left(\mathrm{COCH}_{3}\right), 20.7\left(\mathrm{COCH}_{3}\right)$. HRMS (ES): Calcd. for $\mathrm{C}_{14} \mathrm{H}_{21} \mathrm{NO}_{8} \mathrm{SNH}_{4}: 381.1332$; found 381.1332 . Spectral data are in accordance with published literature. ${ }^{12}$

\section{Synthesis of Group B building blocks.}

\section{Synthesis of galacto alkene azid B1}

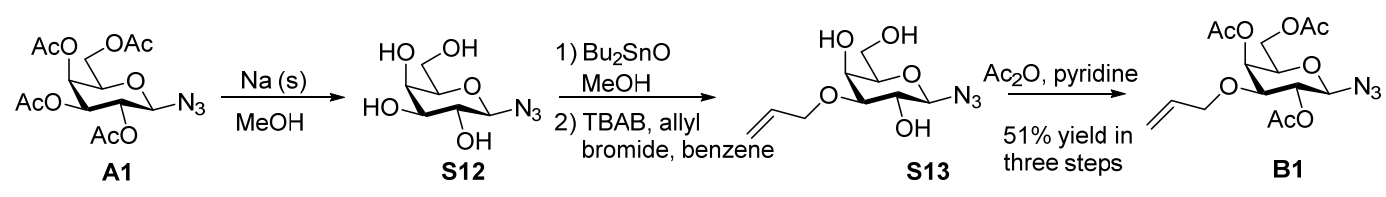

\section{$\beta-D-G a l a c t o p y r a n o s y l$ azide S12}

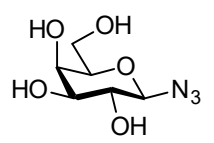

A catalytic amount of metallic sodium was added to a solution of the azide $\mathbf{A} 1(0.203 \mathrm{~g} ; 0.543 \mathrm{mmol} ; 1$ equiv.) dissolved in dry methanol $(6 \mathrm{~mL})$ under a $\mathrm{N}_{2}$ atm. When the reaction had reached completion the methanol was evaporated under reduced pressure. The crude product $\mathbf{S} 12$ was used in the following reaction without further purification. $R_{\mathrm{f}} 0.48$ (ethyl acetate/methanol $\left.3: 1\right)^{1} \mathrm{H} \mathrm{NMR}\left(400 \mathrm{MHz}, d_{3}\right.$ MeOD) $\delta_{H} 4.64(d, J=9.3 \mathrm{~Hz}, 1 \mathrm{H}, \mathrm{H} 1), 3.94(\mathrm{~d}, \mathrm{~J}=3.3 \mathrm{~Hz}, 1 \mathrm{H}, \mathrm{H} 4), 3.84-3.69(\mathrm{~m}, 3 \mathrm{H}, \mathrm{H} 5$ and H6), 3.66 (dd, J = 9.3, 3.3 Hz, $1 \mathrm{H}, \mathrm{H} 3$ ), 3.49 (t, $J=9.3 \mathrm{~Hz}, 1 \mathrm{H}, \mathrm{H} 2$ ). ${ }^{13} \mathrm{C}$ NMR (101 MHz, $\left.d_{3}-\mathrm{MeOD}\right) \delta_{\mathrm{C}} 92.7$ (C1), 78.1 (C5), 75.3 (C3), 72.2 (C2), 70.4 (C4), 60.2.5 (C6). HRMS (ES): Calcd. for $\mathrm{C}_{6} \mathrm{H}_{11} \mathrm{~N}_{3} \mathrm{O}_{5} \mathrm{Na:}$ 228.0596; found 228.0591. Data are in accordance with published literature. ${ }^{6}$ 


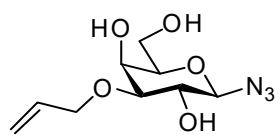

$\mathrm{Bu}_{2} \mathrm{SnO}$ (1.64 g; $6.60 \mathrm{mmol} ; 1.1$ equiv.) was added to a solution of azide $\mathbf{S 1 2}$ (1.23 g; $6.00 \mathrm{mmol} ; 1$ equiv.) dissolved in dry methanol $(50 \mathrm{~mL})$ under a $\mathrm{N}_{2}$ atm. The mixture was heated to reflux for three hours, after which the methanol was evaporated, and the residue was re-dissolved in dry benzene $(37 \mathrm{~mL})$ under a $\mathrm{N}_{2}$ atm. TBAB (1.93 g; $6.00 \mathrm{mmol} ; 1$ equiv.) and allyl bromide (1.65 mL; $18.0 \mathrm{mmol}$; 3 equiv.) was added and the mixture was heated to reflux overnight. When the reaction had reached completion it was cooled to rt, and benzene was evaporated. The crude product $\mathbf{S} 13$ was acetylated without further purification. $R_{\mathrm{f}} 0.58$ (ethyl acetate/methanol 3:1).

\section{2,4,6-Tri-O-acetyl-3-O-allyl- $\beta$-D-galactopyranosyl azide B1}

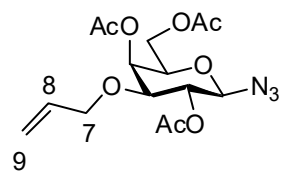

The crude product $\mathbf{S} 13$ was dissolved in acetic anhydride $(4 \mathrm{~mL})$ and dry pyridine $(4 \mathrm{~mL})$ under a $\mathrm{N}_{2}$ atm. After 30 minutes the reaction had reached completion and was quenched by careful addition of $\mathrm{H}_{2} \mathrm{O}$. The reaction mixture was left for 20 minutes before it was diluted with ethyl acetate. The organic phase was washed with $1 \mathrm{M} \mathrm{HCl}(\mathrm{aq})$ and brine, dried over magnesium sulfate, filtered and evaporated. The product B1 was purified by column chromatography (pentane/ethyl acetate 3:1) and isolated as yellow oil in 51\% yield in three

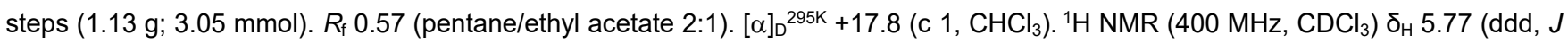
$=22.2,10.7,5.5 \mathrm{~Hz}, 1 \mathrm{H}, \mathrm{H} 8), 5.44(\mathrm{~d}, \mathrm{~J}=3.2 \mathrm{~Hz}, 1 \mathrm{H}, \mathrm{H} 4), 5.32-5.13(\mathrm{~m}, 2 \mathrm{H}, \mathrm{H} 9), 5.05(\mathrm{t}, \mathrm{J}=9.4 \mathrm{~Hz}, 1 \mathrm{H}, \mathrm{H} 2), 4.49(\mathrm{~d}, \mathrm{~J}=9.4 \mathrm{~Hz}$, $1 \mathrm{H}, \mathrm{H} 1), 4.24-4.06(\mathrm{~m}, 3 \mathrm{H} ; \mathrm{H} 5$ and H6), $3.97-3.85(\mathrm{~m}, 2 \mathrm{H}, \mathrm{H} 7), 3.53(\mathrm{dd}, \mathrm{J}=9.4,3.2 \mathrm{~Hz}, 1 \mathrm{H}, \mathrm{H} 3), 2.13(\mathrm{~s}, 3 \mathrm{H}, \mathrm{COCH}), 2.09(\mathrm{~s}, 3 \mathrm{H}$, $\left.\mathrm{COCH}_{3}\right), 2.06\left(\mathrm{~s}, 3 \mathrm{H}, \mathrm{COCH}_{3}\right) \cdot{ }^{13} \mathrm{C} \mathrm{NMR}\left(101 \mathrm{MHz}, \mathrm{CDCl}_{3}\right) \delta_{\mathrm{C}} 170.6\left(\mathrm{COCH}_{3}\right), 170.2\left(\mathrm{COCH}_{3}\right), 169.5\left(\mathrm{COCH}_{3}\right), 133.9(\mathrm{C} 8), 117.6(\mathrm{C} 9)$, 88.4 (C1), 76.5 (C3), 73.2 (C7), 70.7, 69.7 (C2 and C5), 66.0 (C4), 62.0 (C6), $20.9\left(\mathrm{COCH}_{3}\right), 20.8\left(\mathrm{COCH}_{3}\right)$. HRMS (ES): Calcd. for $\mathrm{C}_{15} \mathrm{H}_{21} \mathrm{~N}_{3} \mathrm{O}_{8} \mathrm{Na}$ : 394.1226; found. 394.1224.

\section{Synthesis of GIcNAc alkene azide B2}

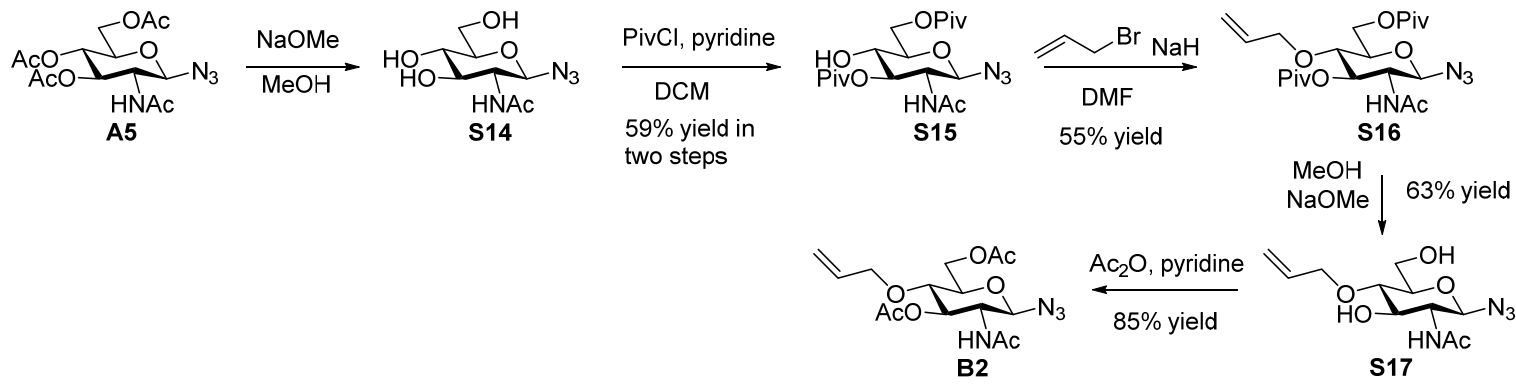

\section{2-Acetamido-2-deoxy- $\beta$-D-glucopyranosyl azide S14}

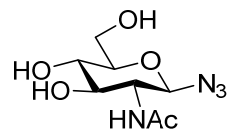

A sodium methoxide solution was prepared by dissolving a catalytic amount of metallic sodium in dry methanol (2 mL) under a $\mathrm{N}_{2}$ atm. $1 \mathrm{~mL}$ of the sodium methoxide solution was then added to a solution of the per-acetylated GlcNAc azide A5 (0.587 $\mathrm{g} ; 1.58 \mathrm{mmol} ; 1$ 
equiv) dissolved in dry methanol $(5 \mathrm{~mL})$ under a $\mathrm{N}_{2}$ atm. When the reaction had reached completion, methanol was evaporated and the product was used in the following reaction without further purification.

\section{2-Acetamido-2-deoxy-3,6-tri-O-pivaloyl- $\beta$-D-glucopyranosyl azide S15}

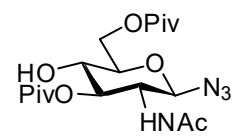

The GlcNAc azide $\mathbf{S} 14$ (0.388 g; $1.58 \mathrm{mmol}$; 1 equiv.) was dissolved in dry dichloromethane (2.8 $\mathrm{mL})$ and dry pyridine (6.7 $\mathrm{mL})$ under a $\mathrm{N}_{2}$ atm. The solution was then cooled to $0{ }^{\circ} \mathrm{C}$ before pivaloyl chloride $(0.54 \mathrm{~mL} ; 4.41 \mathrm{mmol} ; 2.8$ equiv.) was added dropwise. The reaction was then stirred at $0{ }^{\circ} \mathrm{C}$ for three hours, until TLC analysis showed that the reaction had reached completion. The reaction mixture was then diluted with dichloromethane, and the organic phase was washed sequentially with sat. $\mathrm{NaHCO}_{3}(\mathrm{aq}), \mathrm{H}_{2} \mathrm{O}$ and brine, dried over magnesium sulfate, filtered and evaporated. The product $\mathbf{S} 15$ was purified by column chromatography (pentane/ethyl acetate $2: 1)$ and obtained as a white solid in $59 \%$ yield in two steps $(0.387 \mathrm{~g} ; 0.934 \mathrm{mmol})$. $R_{\mathrm{f}} 0.50$ (pentane/ethyl acetate $\left.2: 1\right)$. [ $\left.\alpha\right]_{D}{ }^{295 K}-72.4$ (c 1, $\mathrm{CHCl}_{3}$ ) [Lit.: -19 $\left(\mathrm{c}_{0.2}, \mathrm{CHCl}_{3}\right)^{19}{ }^{1} \mathrm{H}$ NMR $\left(400 \mathrm{MHz}, \mathrm{CDCl}_{3}\right) \delta_{\mathrm{H}} 6.14(\mathrm{~d}, \mathrm{~J}=9.4 \mathrm{~Hz}, 1 \mathrm{H}, \mathrm{NH}), 5.13(\mathrm{dd}, \mathrm{J}=10.4,9.3 \mathrm{~Hz}, 1 \mathrm{H}, \mathrm{H} 3)$, $4.58(\mathrm{~d}, \mathrm{~J}=9.3 \mathrm{~Hz}, 1 \mathrm{H}, \mathrm{H} 1$ ), 4.44 (dd, J = 12.2, $4.3 \mathrm{~Hz}, 1 \mathrm{H}, \mathrm{H} 6 \mathrm{a}), 4.37$ (dd, J = 12.2, $2.4 \mathrm{~Hz}, 1 \mathrm{H}, \mathrm{H} 6 \mathrm{~b}), 3.98$ (q, J = 9.3 Hz, 1H, H2), 3.66 (ddd, $\mathrm{J}=9.3,4.3,2.4 \mathrm{~Hz}, 1 \mathrm{H}, \mathrm{H} 5), 3.52$ (t, J = 9.3 Hz, 1H, H4), 3.38 (br s, $1 \mathrm{H}, \mathrm{OH}$ ), 1.95 (s, 3H, NCOCH 3 ), $1.23(\mathrm{~s}, 9 \mathrm{H}, 3 \mathrm{CH}$ ), $1.19\left(\mathrm{~s}, 9 \mathrm{H}, 3 \mathrm{CH}_{3}\right) .{ }^{13} \mathrm{C} \mathrm{NMR}\left(101 \mathrm{MHz}, \mathrm{CDCl}_{3}\right) \delta_{\mathrm{C}} 180.0\left(\mathrm{COOC}\left(\mathrm{CH}_{3}\right)_{3}\right), 179.6\left(\mathrm{COOC}\left(\mathrm{CH}_{3}\right)_{3}\right), 170.5\left(\mathrm{NCOCH}_{3}\right), 88.8(\mathrm{C} 1), 76.5(\mathrm{C} 5)$, 74.5 (C3), 68.9 (C4), 62.8 (C6), $53.6(\mathrm{C} 2), 39.2\left(\mathrm{OC}\left(\mathrm{CH}_{3}\right)_{3}\right), 27.3\left(\mathrm{OC}\left(\mathrm{CH}_{3}\right)_{3}\right), 27.1\left(\mathrm{OC}\left(\mathrm{CH}_{3}\right)_{3}\right), 23.3\left(\mathrm{NCOCH}_{3}\right)$. HRMS (ES): Calcd. for $\mathrm{C}_{18} \mathrm{H}_{30} \mathrm{~N}_{4} \mathrm{O}_{7} \mathrm{H}:$ 415.2193; found. 415.2193. Spectral data are not in accordance with literature values. ${ }^{19}$

\section{2-Acetamido-2-deoxy-4-O-allyl-3,6-di-O-pivaloyl- $\beta$-D-glucopyranosyl azide S16}

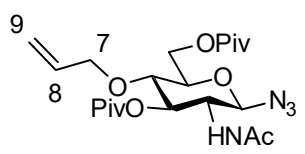

$\mathrm{NaH}$ (60\% in mineral oil) $(0.080 \mathrm{~g} ; 1.99 \mathrm{mmol} ; 1.05$ equiv.) was added to a solution of the di-pivaloyalated GlcNAc azide S15 (0.788 g; 1.90 mmol; 1 equiv.) and allyl bromide ( $0.19 \mathrm{ml} ; 2.28 \mathrm{mmol} ; 1.05$ equiv.) dissolved in dry DMF (20 mL) under a $\mathrm{N}_{2}$ atm. After 1.5 hours the reaction had reached completion and was quenched by addition of $\mathrm{H}_{2} \mathrm{O}$. The mixture was diluted with ethyl acetate and the organic phase was washed with $\mathrm{H}_{2} \mathrm{O}$ and brine, dried over magnesium sulfate, filtered and evaporated. The product $\mathbf{S} 16$ was purified by column chromatography (pentane/ethyl acetate 3:1) and obtained as clear oil in $55 \%$ yield $(0.4738 \mathrm{~g} ; 1.04 \mathrm{mmol})$. $R_{\mathrm{f}} 0.30$ (pentane/ethyl acetate 2:1). $[\alpha]_{D}^{295 K}-57.4\left(c 1, \mathrm{CHCl}_{3}\right) .{ }^{1} \mathrm{H}$ NMR $\left(400 \mathrm{MHz}, \mathrm{CDCl}_{3}\right) \delta_{\mathrm{H}} 6.43(\mathrm{~d}, \mathrm{~J}=9.8 \mathrm{~Hz}, 1 \mathrm{H}, \mathrm{NH}), 5.74(\mathrm{ddt}, \mathrm{J}=17.2,10.6,5.7 \mathrm{~Hz}$, $1 \mathrm{H}, \mathrm{H} 8$ ), 5.22-5.06 (m, 3H, H3 and H9), 4.43-4.38 (m, 2H, H1 and H6a), 4.21 - 3.93 (m, 4H, H2, H6b, H7), 3.70 (ddd, J = 9.4, 4.5, 2.0 $\mathrm{Hz}, 1 \mathrm{H}, \mathrm{H} 5), 3.51(\mathrm{t}, \mathrm{J}=9.4 \mathrm{~Hz}, 1 \mathrm{H}, \mathrm{H} 4), 1.92\left(\mathrm{~s}, 3 \mathrm{H}, \mathrm{NCOCH}_{3}\right), 1.20\left(\mathrm{~s}, 9 \mathrm{H}, 3 \mathrm{CH}_{3}\right), 1.18\left(\mathrm{~s}, 9 \mathrm{H}, 3 \mathrm{CH}_{3}\right) .{ }^{13} \mathrm{C} \mathrm{NMR}\left(101 \mathrm{MHz}^{\mathrm{C} C D C l}\right)$ $\delta_{\mathrm{C}} 179.5\left(\mathrm{COOC}\left(\mathrm{CH}_{3}\right)_{3}\right), 178.1\left(\mathrm{COOC}\left(\mathrm{CH}_{3}\right)_{3}\right), 170.2\left(\mathrm{NCOCH}_{3}\right), 133.5(\mathrm{C} 8), 118.1$ (C9), $88.8(\mathrm{C} 1), 75.8$ (C4), 75.1 (C3), 75.0 (C5), 74.0 (C7), 62.3 (C6), $53.1(\mathrm{C} 2), 39.1\left(\mathrm{OC}\left(\mathrm{CH}_{3}\right)_{3}\right), 39.0\left(\mathrm{OC}\left(\mathrm{CH}_{3}\right)_{3}\right), 27.2\left(\mathrm{OC}\left(\mathrm{CH}_{3}\right)_{3}\right), 27.1\left(\mathrm{OC}\left(\mathrm{CH}_{3}\right)_{3}\right), 23.1(\mathrm{NCOCH})$. HRMS (ES): Calcd. for $\mathrm{C}_{21} \mathrm{H}_{34} \mathrm{~N}_{4} \mathrm{O}_{7} \mathrm{Na}$ : 477.2325; found. 477.2324.

\section{2-Acetamido-2-deoxy-4-O-allyl- $\beta$-D-glucopyranosyl azide S17}

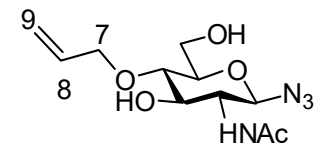

The allylated GlcNAc azide S16 (0.474 g; 1.04 mmol; 1 equiv.) was dissolved in dry methanol (10 mL) under a $\mathrm{N}_{2}$ atm. Sodium methoxide solution ( $25 \%$ in methanol) was then added dropwise until pH 10 was reached. The reaction was then left overnight. When 
the reaction had reached completion methanol was evaporated and product $\mathbf{S} 17$ was purified by column chromatography (ethyl acetate/pentane/methanol 2:1:0.1) and obtained as a white foam in $63 \%$ yield $\left(0.188 \mathrm{~g} ; 0.655 \mathrm{mmol}\right.$ ). $R_{\mathrm{f}} 0.30$ (ethyl acetate/pentane/methanol 2:1:0.1). [ $\alpha]_{D}{ }^{295 K}-21.2(c 1, \mathrm{MeOH}) .{ }^{1} \mathrm{H}$ NMR $\left(400 \mathrm{MHz}, d_{3}-\mathrm{MeOD}\right) \delta_{\mathrm{H}} 5.94(\mathrm{ddt}, \mathrm{J}=17.2,10.6,5.6 \mathrm{~Hz}, 1 \mathrm{H}$, H8), 5.25 (dd, J = 17.2, 1.8 Hz, 1H, H9a), 5.11 (dd, J = 10.6, $1.8 \mathrm{~Hz}, 1 \mathrm{H}, \mathrm{H} 9 \mathrm{~b}$ ), 4.49 (d, J = 9.8 Hz, 1H, H1), 4.39 (dd, J = 12.5, $5.6 \mathrm{~Hz}$, $1 \mathrm{H}, \mathrm{H} 7 \mathrm{a}$ ), 4.15 (dd, J = 12.5, 5.6 Hz, 1H, H7b), 3.86 (dd, J = 12.1, 1.9 Hz, 1H, H6a), 3.70 (dd, J = 12.1, 4.8 Hz, 1H, H6b), 3.68 (t, J = $9.8 \mathrm{~Hz}, 1 \mathrm{H}, \mathrm{H} 2$ ), 3.57 (dd, J = 9.8, $8.5 \mathrm{~Hz}, 1 \mathrm{H}, \mathrm{H} 3$ ), 3.37 (ddd, J = 9.7, 4.8, $1.9 \mathrm{~Hz}, 1 \mathrm{H}, \mathrm{H} 5$ ), 3.33-3.26 (m, 1H, H4), 1.98 (s, 3H, NCOCH $)$. ${ }^{13} \mathrm{C}$ NMR (101 MHz, $\left.d_{3}-\mathrm{MeOD}\right) \delta_{\mathrm{C}} 173.8\left(\mathrm{NCOCH}_{3}\right), 136.4$ (C8), 116.9 (C9), 90.1 (C1), 79.5 (C4), 79.0 (C5), 76.0 (C3), 74.8 (C7), 62.1 (C6), 57.0 (C2), $22.9\left(\mathrm{NCOCH}_{3}\right)$. HRMS (ES): Calcd. for $\mathrm{C}_{11} \mathrm{H}_{18} \mathrm{~N}_{4} \mathrm{O}_{5} \mathrm{H}: 287.1355$; found. 287.1351.

\section{2-Acetamido-2-deoxy-3,6-di-O-acetyl-4-O-allyl- $\beta$-D-glucopyranosyl azide B2}

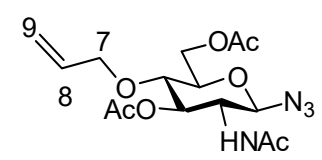

The GlcNAc azide $\mathbf{S} 17$ (0.141 g; 0.491 mmol; 1 equiv.) was dissolved in dry pyridine (2 mL) and acetic anhydride $(2 \mathrm{~mL})$ under a $\mathrm{N}_{2}$ atm. After 4 hours the reaction had reached completion and was carefully quenched by the addition of $\mathrm{H}_{2} \mathrm{O}$. The reaction mixture was left for 20 minutes before it was diluted with ethyl acetate. The organic phase was washed with $1 \mathrm{M} \mathrm{HCl}(\mathrm{aq}), \mathrm{H}_{2} \mathrm{O}, \mathrm{sat} \mathrm{NaHCO}_{3}(\mathrm{aq})$ and brine, dried over magnesium sulfate, filtered and evaporated. The product B2 was isolated as clear oil in $85 \%$ yield $(0.155 \mathrm{~g} ; 0.417$ mmol). $R_{\mathrm{f}} 0.50$ (ethyl acetate). $[\alpha]_{\mathrm{D}}{ }^{295 \mathrm{~K}}-51.8\left(\mathrm{c} 1, \mathrm{CHCl}_{3}\right) .{ }^{1} \mathrm{H} \mathrm{NMR}\left(400 \mathrm{MHz}, \mathrm{CDCl}_{3}\right) \delta_{\mathrm{H}} 5.89(\mathrm{~d}, \mathrm{~J}=9.2 \mathrm{~Hz}, 1 \mathrm{H}, \mathrm{NH}), 5.79(\mathrm{ddt}, \mathrm{J}=$ $16.1,10.4,5.8 \mathrm{~Hz}, 1 \mathrm{H}, \mathrm{H} 8), 5.27-5.12(\mathrm{~m}, 2 \mathrm{H}, \mathrm{H} 9), 5.06(\mathrm{dd}, \mathrm{J}=10.7,9.1 \mathrm{~Hz}, 1 \mathrm{H}, \mathrm{H} 3), 4.48(\mathrm{~d}, \mathrm{~J}=9.2 \mathrm{~Hz}, 1 \mathrm{H}, \mathrm{H} 1), 4.40(\mathrm{dd}, \mathrm{J}=$ 12.1, $2.1 \mathrm{~Hz}, 1 \mathrm{H}, \mathrm{H} 6 \mathrm{a}$ ), 4.23 (dd, J = 12.1, 4.7 Hz, 1H, H6b), $4.12-4.01$ (m, 3H, H2 and H7), 3.65 (ddd, J = 9.1, 4.7, 2.1 Hz, 1H, H5), $3.55(\mathrm{t}, \mathrm{J}=9.1 \mathrm{~Hz}, 1 \mathrm{H}, \mathrm{H} 4), 2.11\left(\mathrm{~s}, 3 \mathrm{H}, \mathrm{COCH}_{3}\right), 2.10\left(\mathrm{~s}, 3 \mathrm{H}, \mathrm{COCH}_{3}\right), 1.97\left(\mathrm{~s}, 3 \mathrm{H}, \mathrm{COCH}_{3}\right) \cdot{ }^{13} \mathrm{C} \mathrm{NMR}\left(101 \mathrm{MHz} \mathrm{CDCl}_{3}\right) \delta_{\mathrm{C}} 171.5$ $\left(\mathrm{COCH}_{3}\right), 170.8\left(\mathrm{COCH}_{3}\right), 170.5\left(\mathrm{COCH}_{3}\right), 133.8(\mathrm{C} 8), 118.2(\mathrm{C} 9), 88.8(\mathrm{C} 1), 75.3,75.2,75.2$ (C3, C4 and C5), 74.0 (C7), $62.6(\mathrm{C} 6)$, $53.5(\mathrm{C} 2), 23.3\left(\mathrm{COCH}_{3}\right), 21.1\left(\mathrm{COCH}_{3}\right), 20.9\left(\mathrm{COCH}_{3}\right)$. HRMS (ES): Calcd. for $\mathrm{C}_{15} \mathrm{H}_{22} \mathrm{~N}_{4} \mathrm{O}_{7} \mathrm{H}: 371.1567$; found. 371.1565.

\section{Synthesis of manno alkene azide B3}
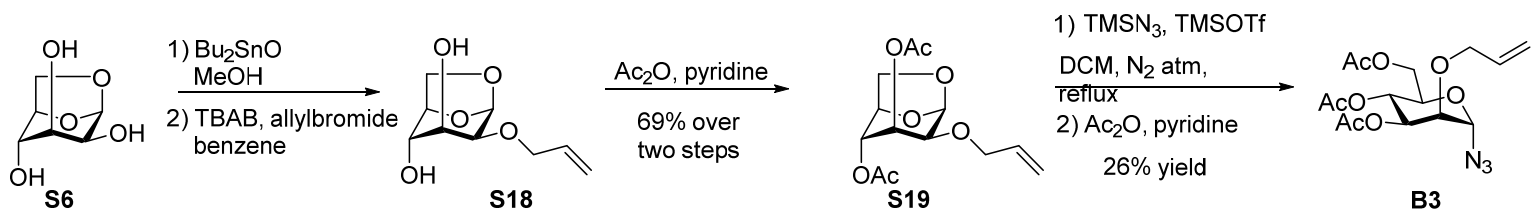

\section{1,6-anhydro-2-O-allyl- $\beta$-D-mannopyranose $S 18$}

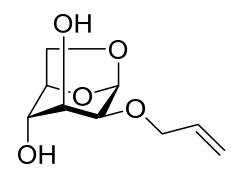

$\mathrm{Bu}_{2} \mathrm{SnO}$ (0.674 g; $2.70 \mathrm{mmol} ; 1.1$ equiv.) was added to a solution of mannosan $\mathbf{S 6}$ (0.398 g; $2.46 \mathrm{mmol} ; 1$ equiv.) dissolved in dry benzene $(10 \mathrm{~mL})$ under a $\mathrm{N}_{2}$ atm. The mixture was heated to reflux for two hours, after which TBAl $(0.909 \mathrm{~g} ; 2.46 \mathrm{mmol} ; 1 \mathrm{equiv}$.) and allyl bromide ( $0.62 \mathrm{~mL} ; 7.37 \mathrm{mmol} ; 3$ equiv.) was added and the mixture was heated to reflux overnight. When the reaction had reached completion it was cooled to rt, and benzene was evaporated. The crude product $\mathbf{S} 18$ was acetylated without further purification.

\section{1,6-anhydro-3,4-O-acetyl-2-O-allyl- $\beta$-D-mannopyranose S19}

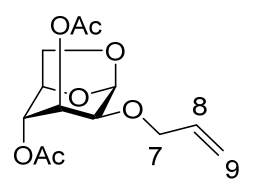


The allylated mannosan $\mathbf{S 1 8}$ was dissolved in dry pyridine $(3 \mathrm{~mL})$ and acetic anhydride $(3 \mathrm{~mL})$ under a $\mathrm{N}_{2}$ atm. and left overnight. When the reaction had reached completion it was carefully quenched by the addition of $\mathrm{H}_{2} \mathrm{O}$ and left for 20 minutes before it was diluted with ethyl acetate. The organic phase was washed with $1 \mathrm{M} \mathrm{HCl}(\mathrm{aq}), \mathrm{H}_{2} \mathrm{O}$, sat $\mathrm{NaHCO}_{3}(\mathrm{aq})$ and brine, dried over magnesium sulfate, filtered and evaporated. The product $\mathbf{S} 19$ was purified by column chromatography (dichloromethane/ethyl acetate 50:1) and isolated as clear oil in $69 \%$ over two steps $(0.488 \mathrm{~g} ; 1.70 \mathrm{mmol}) . R_{\mathrm{f}} 0.42$ (pentane/ethyl acetate 10:1). [ $\left.\alpha\right]_{D}{ }^{295 \mathrm{~K}}-87.8\left(\mathrm{c} 1, \mathrm{CHCl}_{3}\right) .{ }^{1} \mathrm{H} \mathrm{NMR}(400$ $\mathrm{MHz}, \mathrm{CDCl}_{3}$ ) $\delta_{\mathrm{H}} 5.82$ (ddd, $\mathrm{J}=21.8,10.6,5.7 \mathrm{~Hz}, 1 \mathrm{H}, \mathrm{H} 8$ ), $5.41(\mathrm{~s}, 1 \mathrm{H}, \mathrm{H} 1$ ), 5.26 (dd, J=3.1, $1.7 \mathrm{~Hz}, 1 \mathrm{H}, \mathrm{H} 3$ ), 5.24 (ddd, $\mathrm{J}=21.8,3.0$, $1.4 \mathrm{~Hz}, 1 \mathrm{H}, \mathrm{H} 9 \mathrm{a}), 5.16$ (dd, J = 10.6, $1.4 \mathrm{~Hz}, 1 \mathrm{H}, \mathrm{H} 9 \mathrm{~b}), 4.74$ (s, 1H, H4), 4.54 (dd, J = 5.8, 0.9 Hz, 1H, H5), 4.16 (dd, J = 7.7, $0.9 \mathrm{~Hz}$, $1 \mathrm{H}, \mathrm{H6a}$ ), 4.04 (ddt, J = 12.8, 5.7, $1.4 \mathrm{~Hz}, 1 \mathrm{H}, \mathrm{H} 7 \mathrm{a}$ ), 3.95 (ddt, J=12.8, 5.7, 1.2 Hz, 1H, H7b), 3.80 (dd, J = 7.7, 5.8 Hz, 1H, H6b), 3.53 $(\mathrm{dd}, \mathrm{J}=5.5,1.7 \mathrm{~Hz}, 1 \mathrm{H}, \mathrm{H} 2), 2.11\left(\mathrm{~s}, 3 \mathrm{H}, \mathrm{COCH}_{3}\right), 2.11\left(\mathrm{~s}, 3 \mathrm{H}, \mathrm{COCH}_{3}\right) .{ }^{13} \mathrm{C} \mathrm{NMR}\left(101 \mathrm{MHz}, \mathrm{CDCl}_{3}\right) \delta_{\mathrm{C}} 169.8\left(\mathrm{COCH}_{3}\right), 169.7\left(\mathrm{COCH}_{3}\right)$, 134.1 (C8), 118.0 (C9), 100.5 (C1), 73.7 (C5), 72.3 (C2), 72.1 (C4), 71.2 (C7), 67.5 (C3), 65.1 (C6), $21.0\left(\mathrm{COCH}_{3}\right), 21.0\left(\mathrm{COCH}_{3}\right)$. HRMS (ES): Calcd. for $\mathrm{C}_{13} \mathrm{H}_{18} \mathrm{O}_{7} \mathrm{Na}$ : 309.0950; found. 309.0948.

\section{3,4,6-Tri-O-acetyl-2-O-allyl- $\alpha$-D-mannosyl azide B3}

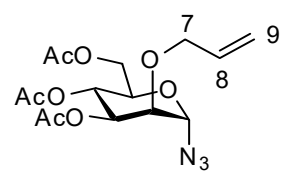

TMSOTf (0.17 mL; $0.939 \mathrm{mmol} ; 1$ equiv.) was added dropwise to a solution of protected allyl mannosan $\mathbf{S 1 9}$ (0.269 g; $0.939 \mathrm{mmol} ; 1$ equiv.) and $\mathrm{TMSN}_{3 \mathrm{j}}\left(1.11 \mathrm{~mL} ; 8.45 \mathrm{mmol} ; 9\right.$ equiv.) dissolved in dry dichloromethane $(17 \mathrm{~mL})$ under a $\mathrm{N}_{2}$ atm. The mixture was heated to reflux and left for three days at reflux and then three days at rt. When the reaction had reached completion dry pyridine $(2 \mathrm{~mL})$ and acetic anhydride $(2 \mathrm{~mL})$ were added under a $\mathrm{N}_{2}$ atm. The acetylation reaction was left overnight. When the reaction had reached completion it was quenched by dropwise addition of $\mathrm{H}_{2} \mathrm{O}$. After 20 min the mixture was diluted with ethyl acetate and the organic phase was washed with $1 \mathrm{M} \mathrm{HCl}(\mathrm{aq}), \mathrm{H}_{2} \mathrm{O}$, sat $\mathrm{NaHCO}_{3}$ and brine sequentially. The organic phase was then dried over magnesium sulfate, filtered and evaporated. The product B3 was purified by column chromatography (pentane/ethyl acetate 4:1) and obtained as light yellow oil in $26 \%$ yield $(0.0891 \mathrm{~g} ; 0.240 \mathrm{mmol}) . R_{\mathrm{f}} 0.66$ (pentane/ethyl acetate $\left.1: 1\right)$. $[\alpha]_{\mathrm{D}}{ }^{295 \mathrm{~K}}+86.6\left(c 1, \mathrm{CHCl}_{3}\right) .{ }^{1} \mathrm{H} \mathrm{NMR}(400 \mathrm{MHz}$, $\left.\mathrm{CDCl}_{3}\right) \delta_{\mathrm{H}} 5.85$ (ddt, $\mathrm{J}=16.2,10.6,5.8 \mathrm{~Hz}, 1 \mathrm{H}, \mathrm{H} 8$ ), $5.42-5.16(\mathrm{~m}, 4 \mathrm{H}, \mathrm{H} 1, \mathrm{H} 4$ and H9), 5.11 (dd, J = 9.9, 3.2 Hz, 1H, H3), $4.27(\mathrm{dd}$, $\mathrm{J}=12.3,5.4 \mathrm{~Hz}, 1 \mathrm{H}, \mathrm{H} 6 \mathrm{a}), 4.15(\mathrm{dd}, \mathrm{J}=12.3,2.1 \mathrm{~Hz}, 1 \mathrm{H}, \mathrm{H} 6 \mathrm{~b}), 4.12-4.02(\mathrm{~m}, 3 \mathrm{H}, \mathrm{H} 5 \mathrm{and} \mathrm{H}$ ) $3.76-3.65(\mathrm{~m}, 1 \mathrm{H}, \mathrm{H} 2), 2.09(\mathrm{~s}, 3 \mathrm{H}$, $\left.\left.\mathrm{COCH}_{3}\right), 2.05\left(\mathrm{~s}, 3 \mathrm{H}, \mathrm{COCH}_{3}\right), 2.03 \mathrm{~s}, 3 \mathrm{H}, \mathrm{COCH}_{3}\right) .{ }^{13} \mathrm{C} \mathrm{NMR}\left(101 \mathrm{MHz}, \mathrm{CDCl}_{3}\right) \delta_{\mathrm{C}} 170.8\left(\mathrm{COCH}_{3}\right), 170.2\left(\mathrm{COCH}_{3}\right), 169.7\left(\mathrm{COCH}_{3}\right)$, 134.0 (C8), 118.4 (C9), 88.1 (C1, J $\mathrm{C}_{\mathrm{C} 1 \mathrm{H} 1}=168.9 \mathrm{~Hz}$ (characteristic of $\alpha$-anomer)), 74.9 (C2), 72.6 (C7), 70.9 (C5), 70.5 (C3), 66.1 (C4), 62.4 (C6), $20.9\left(\mathrm{COCH}_{3}\right), 20.8\left(\mathrm{COCH}_{3}\right), 20.8\left(\mathrm{COCH}_{3}\right)$. HRMS (ES): Calcd. for $\mathrm{C}_{15} \mathrm{H}_{21} \mathrm{~N}_{3} \mathrm{O}_{8} \mathrm{NH}_{4}: 389.1672$; found. 389.1674.

\section{Synthesis of Group C building blocks}

\section{Synthesis of galacto alkyne thioacetate $\mathrm{C} 1$}
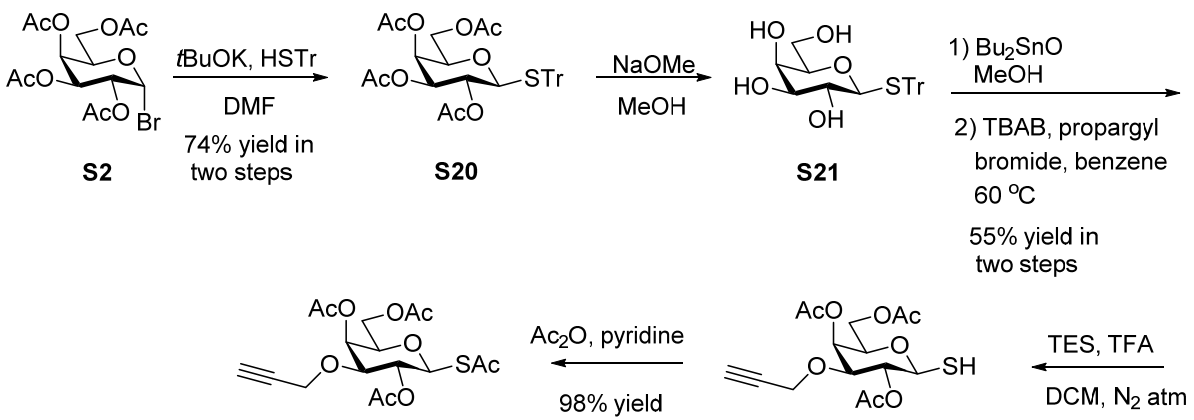

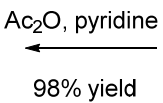

C1

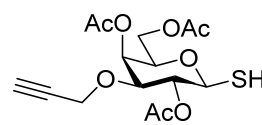

S24
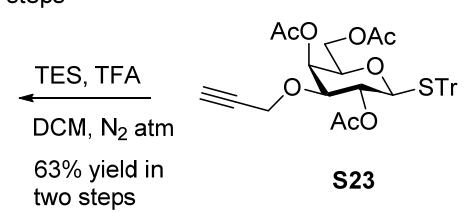

Triphenylmethyl 1-thio-2,3,4,6-tetra-O-acetyl- $\beta$-D-galactopyranoside S20 


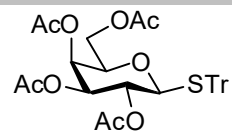

Potassium tert-butoxide (1.75 g; $15.6 \mathrm{mmol} ; 1.2$ equiv.) dissolved in dry DMF ( $8 \mathrm{~mL}$ ) was added dropwise to a solution of triphentlmethyl mercaptan (4.32 g; 15.6 mmol; 1.2 equiv.) dissolved in dry DMF (8 mL) under a $\mathrm{N}_{2}$ atm. This solution was stirred for 15 minutes before being added dropwise to a solution of the bromide $\mathbf{S 2}$ (5.36 g; $13.0 \mathrm{mmol} ; 1$ equiv.) dissolved in dry DMF (17 mL) under a $\mathrm{N}_{2}$ atm. After 4 hours the reaction had reached completion and was quench by the addition of $\mathrm{H}_{2} \mathrm{O}$. The aqueous phase was extracted with ethyl acetate, and the combined organic phases were washed with $\mathrm{H}_{2} \mathrm{O}$ and brine, dried over magnesium sulfate, filtered and evaporated. The product $\mathbf{S 2 0}$ was purified by column chromatography (pentane/ethyl acetate $4: 1$ ) and obtained as light yellow foam in $74 \%$ yield in two steps (5.81 g; $9.58 \mathrm{mmol}) . R_{\mathrm{f}} 0.26$ (pentane ethyl acetate 3:1). [ $\left.\alpha\right]_{D}{ }^{295 \mathrm{~K}}-11.8\left(c 1, \mathrm{CHCl}_{3}\right) .{ }^{1} \mathrm{H} \mathrm{NMR}(400 \mathrm{MHz}, \mathrm{CDCl})_{3} \delta_{\mathrm{H}} 7.43(\mathrm{t}, \mathrm{J}$ $=9.9 \mathrm{~Hz}, 5 \mathrm{H}, \mathrm{ArH}), 7.36-7.19(\mathrm{~m}, 10 \mathrm{H}, \mathrm{ArH}), 5.31(\mathrm{t}, \mathrm{J}=10.0 \mathrm{~Hz}, 1 \mathrm{H}, \mathrm{H} 2), 5.25(\mathrm{~d}, \mathrm{~J}=3.1 \mathrm{~Hz}, 1 \mathrm{H}, \mathrm{H} 4), 4.76(\mathrm{dd}, J=10.0,3.1 \mathrm{~Hz}$, $1 \mathrm{H}, \mathrm{H} 3$ ), 3.87 (dd, $\mathrm{J}=11.2,6.7 \mathrm{~Hz}, 1 \mathrm{H}, \mathrm{H} 6 \mathrm{a}), 3.80$ (dd, $\mathrm{J}=11.2,6.7 \mathrm{~Hz}, 1 \mathrm{H}, \mathrm{H} 6 \mathrm{~b}) .3 .77$ (d, J = 10.0 Hz, 1H, H1), $3.21(\mathrm{t}, J=6.7 \mathrm{~Hz}$, $1 \mathrm{H}, \mathrm{H} 5), 2.15\left(\mathrm{~s}, 3 \mathrm{H}, \mathrm{COCH}_{3}\right), 2.09\left(\mathrm{~s}, 3 \mathrm{H}, \mathrm{COCH}_{3}\right), 1.95\left(\mathrm{~s}, 3 \mathrm{H}, \mathrm{COCH}_{3}\right), 1.91\left(\mathrm{~s}, 3 \mathrm{H}, \mathrm{COCH}_{3}\right) \cdot{ }^{13} \mathrm{C} \mathrm{NMR}\left(101 \mathrm{MHz}, \mathrm{CDCl}_{3}\right) \delta_{\mathrm{C}} 170.3$ $\left(\mathrm{COCH}_{3}\right), 170.2\left(\mathrm{COCH}_{3}\right), 170.1\left(\mathrm{COCH}_{3}\right), 169.4\left(\mathrm{COCH}_{3}\right), 144.4(\mathrm{ArC}), 129.9(\mathrm{ArC}), 127.8(\operatorname{ArC}), 127.0(\operatorname{ArC}), 84.2(\mathrm{C} 1), 74.0(\mathrm{C} 5)$, $72.2(\mathrm{C} 3), 68.8\left(\mathrm{SCPh}_{3}\right), 67.1(\mathrm{C} 2), 66.9(\mathrm{C} 4), 61.0(\mathrm{C} 6), 20.9\left(\mathrm{COCH}_{3}\right), 20.7\left(\mathrm{COCH}_{3}\right), 20.6\left(\mathrm{COCH}_{3}\right), 20.6(\mathrm{COCH})$. HRMS (ES): Calcd. for $\mathrm{C}_{33} \mathrm{H}_{34} \mathrm{O}_{9} \mathrm{SNH}_{4}$ : 624.2267 ; found. 624.2267 .

\section{Triphenylmethyl 1-thio- $\beta-D-g a l a c t o p y r a n o s i d e ~ S 21$}

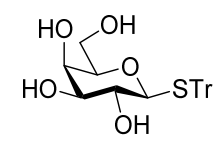

A sodium methoxide solution was prepared by dissolving a catalytic amount of metallic sodium in dry methanol $(2 \mathrm{~mL}) \mathrm{under}$ a $\mathrm{N}_{2}$ atm. $1 \mathrm{~mL}$ of the sodium methoxide solution was then added to a solution of the peracetylated tritylthiol S20 (2.42 g; $3.98 \mathrm{mmol} ; 1 \mathrm{equiv}$.) dissolved in dry methanol $(15 \mathrm{~mL})$ under a $\mathrm{N}_{2}$ atm. When the reaction had reached completion, methanol was evaporated and the product $\mathbf{S 2 1}$ was used in the following reaction without further purification.

\section{Triphenylmethyl 1-thio-2,4,6-tri-O-acetyl-3-propargyl- $\beta$-D-galactopyranoside S22}

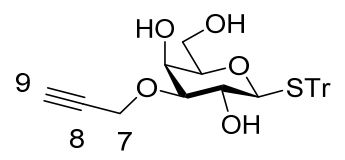

$\mathrm{Bu}_{2} \mathrm{SnO}$ (1.70 g; $6.82 \mathrm{mmol} ; 1.1$ equiv.) was added to a solution of deprotected galactosyl tritylthiol S21 (2.72 g; $6.19 \mathrm{mmol} ; 1$ equiv.) dissolved in dry methanol $(19 \mathrm{~mL})$ under a $\mathrm{N}_{2}$ atm. The mixture was heated to reflux for three hours, after which the methanol was evaporated, and the residue was re-dissolved in dry benzene $(26 \mathrm{~mL})$ under a $\mathrm{N}_{2}$ atm. TBAB (1.99 g; $6.19 \mathrm{mmol} ; 1$ equiv.) and allyl bromide (1.66 mL; $18.6 \mathrm{mmol} ; 3$ equiv.) was added and the mixture was heated to $60{ }^{\circ} \mathrm{C}$ overnight. When the reaction had reached completion it was cooled to rt, and benzene was evaporated. The product $\mathbf{S} 22$ was purified by column chromatography (dichloromethane/methanol 100:1) and isolated as a light yellow foam in 55\% yield in two steps (1.62 $\mathrm{g} ; 3.40 \mathrm{mmol}$ ). $R_{\mathrm{f}} 0.49$ (ethyl acetate/pentane 2:1). $[\alpha]_{D}{ }^{295 K}-84.8\left(c\right.$ 1, $\left.\mathrm{CHCl}_{3}\right) .{ }^{1} \mathrm{H}$ NMR $\left(400 \mathrm{MHz}, \mathrm{CDCl}_{3}\right) \delta_{\mathrm{H}} 7.53-7.46(\mathrm{~m}, 6 \mathrm{H}, \mathrm{ArH}), 7.34-7.20(\mathrm{~m}, 9 \mathrm{H}, \mathrm{ArH})$, 4.33 (dd, $J=16.1,2.4 \mathrm{~Hz}, 1 \mathrm{H}, \mathrm{H} 7 \mathrm{a}$ ), 4.25 (dd, $\mathrm{J}=16.1,2.4 \mathrm{~Hz}, 1 \mathrm{H}, \mathrm{H} 7 \mathrm{~b}$ ), 3.95 (dd, J = 3.3, $0.8 \mathrm{~Hz}, 1 \mathrm{H}, \mathrm{H} 4$ ), 3.84 (dd, J = 9.9, $8.8 \mathrm{~Hz}$, $1 \mathrm{H}, \mathrm{H} 2$ ), 3.77 (dd, J = 11.9, 7.3 Hz, 1H, H6a), 3.67 (d, J = 9.9 Hz, 1H, H1), 3.51 (dd, J = 11.9, 4.0 Hz, 1H, H6b), 3.25 (dd, J = 8.8, 3.3 $\mathrm{Hz}, 1 \mathrm{H}, \mathrm{H} 3$ ), 2.95 (ddd, J = 7.3, 4.0, $0.8 \mathrm{~Hz}, 1 \mathrm{H}, \mathrm{H5}$ ), 2.45 (s, 1H, H9). ${ }^{13} \mathrm{C} \mathrm{NMR}\left(101 \mathrm{MHz}, \mathrm{CDCl}_{3}\right) \delta_{\mathrm{C}}$ 144.6, 130.0, 128.0, 127.2 (ArC), 86.1 (C1), 81.9 (C3), 79.7 (C8/C9), 78.3 (C5), 75.3 (C9/C8), 69.4 (C2), 68.7 (SCPh $), 67.4$ (C4), 62.6 (C6), 57.9 (C7). HRMS (ES): Calcd. for $\mathrm{C}_{28} \mathrm{H}_{28} \mathrm{NO}_{5} \mathrm{SNH}_{4}$ : 494.2001; found. 494.1999. 


\section{Triphenylmethyl 1-thio-2,4,6-tri-O-acetyl-3-propargyl- $\beta$-D-galactopyranoside S23}

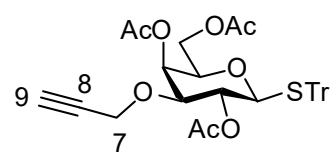

The trityl protected alkyne thiol $\mathbf{S 2 2}$ (3.98 g; $8.35 \mathrm{mmol}$; 1 equiv.) was dissolved in dry pyridine (15 $\mathrm{mL})$ and acetic anhydride (15 $\mathrm{mL}$ ) under a $\mathrm{N}_{2}$ atm. After 4 hours the reaction had reached completion and was carefully quenched by the addition of $\mathrm{H}_{2} \mathrm{O}$. The reaction mixture was left for 20 minutes before it was diluted with ethyl acetate. The organic phase was washed with $1 \mathrm{M} \mathrm{HCl}(\mathrm{aq}), \mathrm{H}_{2} \mathrm{O}, \mathrm{sat}$ $\mathrm{NaHCO}_{3}(\mathrm{aq})$ and brine, dried over magnesium sulfate, filtered and evaporated. The crude product $\mathbf{S 2 3}$ was used in the following reaction without further purification. $R_{\mathrm{f}} 0.68$ (pentane/ethyl acetate $\left.2: 1\right) \cdot[\alpha]_{D}{ }^{295 \mathrm{~K}}+18.4\left(\mathrm{c} 1, \mathrm{CHCl}_{3}\right) .{ }^{1} \mathrm{H} \mathrm{NMR}\left(400 \mathrm{MHz}, \mathrm{CDCl}_{3}\right) \delta_{\mathrm{H}} 7.46$ - $7.39(\mathrm{~m}, 6 \mathrm{H}, \mathrm{ArH}), 7.33-7.19(\mathrm{~m}, 9 \mathrm{H}, \mathrm{ArH}), 5.24(\mathrm{~d}, \mathrm{~J}=3.1 \mathrm{~Hz}, 1 \mathrm{H}, \mathrm{H} 4), 5.14(\mathrm{t}, \mathrm{J}=9.8 \mathrm{~Hz}, 1 \mathrm{H}, \mathrm{H} 2), 4.10(\mathrm{dd}, \mathrm{J}=6.2,2.4 \mathrm{~Hz}, 2 \mathrm{H}$, $\mathrm{H} 7$ ), 3.88 (dd, J = 11.2, $6.9 \mathrm{~Hz}, 1 \mathrm{H}, \mathrm{H} 6 \mathrm{a}$ ), 3.79 (dd, J=11.2, $6.9 \mathrm{~Hz}, 1 \mathrm{H}, \mathrm{H} 6 \mathrm{~b}), 3.76(\mathrm{~d}, \mathrm{~J}=9.8 \mathrm{~Hz}, 1 \mathrm{H}, \mathrm{H} 1$ ), 3.50 (dd, J = 9.8, $3.1 \mathrm{~Hz}$, $1 \mathrm{H}, \mathrm{H} 3), 3.12(\mathrm{t}, \mathrm{J}=6.9 \mathrm{~Hz}, 1 \mathrm{H}, \mathrm{H} 5), 2.39(\mathrm{t}, \mathrm{J}=2.4 \mathrm{~Hz}, 1 \mathrm{H}, \mathrm{H} 9), 2.12\left(\mathrm{~s}, 3 \mathrm{H}, \mathrm{COCH}_{3}\right), 2.12\left(\mathrm{~s}, 3 \mathrm{H}, \mathrm{COCH}_{3}\right), 1.91\left(\mathrm{~s}, 3 \mathrm{H}, \mathrm{COCH}_{3}\right) .{ }^{13} \mathrm{C}$ $\operatorname{NMR}\left(101 \mathrm{MHz}, \mathrm{CDCl}_{3}\right) \delta_{\mathrm{C}} 170.6\left(\mathrm{COCH}_{3}\right), 170.4\left(\mathrm{COCH}_{3}\right), 169.6\left(\mathrm{COCH}_{3}\right), 144.6,130.0,127.8,127.0(\mathrm{ArC}), 84.1(\mathrm{C} 1), 79.2(\mathrm{C} 8 / \mathrm{C} 9)$, 77.3 (C3), 75.0 (C9/C8), 74.1 (C5), $68.6\left(\mathrm{SCPh}_{3}\right), 68.2$ (C2), $65.6(\mathrm{C} 4), 61.5(\mathrm{C} 6), 56.6(\mathrm{C} 7), 21.2\left(\mathrm{COCH}_{3}\right), 20.9\left(\mathrm{COCH}_{3}\right), 20.7$ $\left(\mathrm{COCH}_{3}\right)$. HRMS (ES): Calcd. for $\mathrm{C}_{34} \mathrm{H}_{34} \mathrm{NO}_{8} \mathrm{SNH}_{4}$ : 620.2318; found. 620.2318.

\section{2,4,6-Tri-O-acetyl-3-propargyl- $\beta$-D-galactopyranosyl thiol S24}

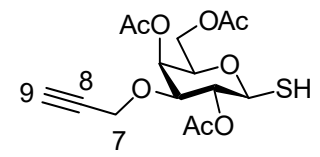

Triethylsilane ( $0.45 \mathrm{~mL} ; 2.82 \mathrm{mmol} ; 2.3$ equiv.) and TFA (3.6 mL) were added to a solution of the alkyne trityl thiol S23 (0.738 $\mathrm{g} ; 1.22$ mmol; 1 equiv.) dissolved in dry dichloromethane $(11 \mathrm{~mL})$ under a $\mathrm{N}_{2}$ atm. After 40 minutes the reaction had reached completion and the solvents were evaporated followed by co-evaporation with toluene. The residue was purified by column chromatography (pentane/ethyl acetate 4:1). The product $\mathbf{S 2 4}$ was obtained as clear oil in $63 \%$ yield in two steps. $R_{\mathrm{f}} 0.43$ (pentane/ethyl acetate $1: 1$ ).

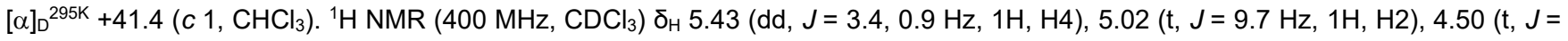
$9.7 \mathrm{~Hz}, 1 \mathrm{H}, \mathrm{H} 1), 4.18$ (d, J = 2.4 Hz, 2H, H7), $4.17-4.08$ (m, 2H, H6), 3.87 (td, J = 6.5, 0.9 Hz, 1H, H5), 3.79 (dd, J=9.7, 3.4 Hz, 1H, $\mathrm{H} 3$ ), $2.44(\mathrm{t}, \mathrm{J}=2.4 \mathrm{~Hz}, 1 \mathrm{H}, \mathrm{H} 9), 2.35(\mathrm{~d}, \mathrm{~J}=9.9 \mathrm{~Hz}, 1 \mathrm{H}, \mathrm{SH}), 2.14\left(\mathrm{~s}, 3 \mathrm{H}, \mathrm{COCH}_{3}\right), 2.12\left(\mathrm{~s}, 3 \mathrm{H}, \mathrm{COCH}_{3}\right), 2.06\left(\mathrm{~s}, 3 \mathrm{H}, \mathrm{COCH}_{3}\right) .{ }^{13} \mathrm{C}$ $\operatorname{NMR}\left(101 \mathrm{MHz}, \mathrm{CDCl}_{3}\right) \delta_{\mathrm{C}} 170.7\left(\mathrm{COCH}_{3}\right), 170.5\left(\mathrm{COCH}_{3}\right), 170.1\left(\mathrm{COCH}_{3}\right), 79.2(\mathrm{C} 1), 79.1$ (C8/C9), $76.5(\mathrm{C} 3), 75.3(\mathrm{C} 5 / \mathrm{C} 8 / \mathrm{C} 9), 75.2$ (C5/C8/C9), 72.2 (C2), 65.9 (C4), 62.2 (C6), 56.7 (C7), $21.1\left(\mathrm{COCH}_{3}\right), 20.9\left(\mathrm{COCH}_{3}\right), 20.9\left(\mathrm{COCH}_{3}\right)$. HRMS (ES): Calcd. for $\mathrm{C}_{15} \mathrm{H}_{20} \mathrm{O}_{8} \mathrm{SNa}$ : 383.0777; found. 383.0773 .

\section{1-S-acetyl-2,4,6-tri-O-acetyl-3-propargyl-1-thio- $\beta$-D-galactopyranose C1}

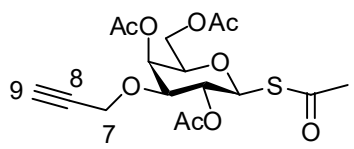

The thiol S24 (0.546 g; 1.51 mmol; 1 equiv.) was dissolved in acetic anhydride ( $3 \mathrm{~mL}$ ) and dry pyridine ( $3 \mathrm{~mL})$ under a $\mathrm{N}_{2}$ atm, and left overnight. When the reaction had reached completion it was carefully quenched by the addition of $\mathrm{H}_{2} \mathrm{O}$. The reaction mixture was left 
for 20 minutes before it was diluted with ethyl acetate. The organic phase was washed with $1 \mathrm{M} \mathrm{HCl}(\mathrm{aq}), \mathrm{H}_{2} \mathrm{O}, \mathrm{sat} \mathrm{NaHCO}_{3}(\mathrm{aq})$ and brine, dried over magnesium sulfate, filtered and evaporated. The product $\mathbf{C} 1$ was isolated as clear oil in $98 \%$ yield $(0.598 \mathrm{~g} ; 1.48$ mmol). $R_{\mathrm{f}} 0.50$ (pentane/ethyl acetate 1:1). $[\alpha]_{\mathrm{D}}{ }^{295 \mathrm{~K}}+57.2\left(\mathrm{c} 1, \mathrm{CHCl}_{3}\right) .{ }^{1} \mathrm{H} \mathrm{NMR}\left(400 \mathrm{MHz}, \mathrm{CDCl}_{3}\right) \delta_{\mathrm{H}} 5.44(\mathrm{dd}, \mathrm{J}=3.4,0.8 \mathrm{~Hz}, 1 \mathrm{H}$, $\mathrm{H} 4), 5.24-5.11(\mathrm{~m}, 2 \mathrm{H}, \mathrm{H} 1$ and H2), $4.18(\mathrm{~d}, \mathrm{~J}=2.4 \mathrm{~Hz}, 2 \mathrm{H}, \mathrm{H} 7), 4.14(\mathrm{dd}, \mathrm{J}=11.3,6.5 \mathrm{~Hz}, 1 \mathrm{H}, \mathrm{H} 6 \mathrm{a}), 4.05(\mathrm{dd}, \mathrm{J}=11.3,6.5 \mathrm{~Hz}, 1 \mathrm{H}$, $\mathrm{H6b}$ ), 3.98 (td, $\mathrm{J}=6.5,0.8 \mathrm{~Hz}, 1 \mathrm{H}, \mathrm{H} 5$ ), 3.89 (dd, J = 9.2, 3.4 Hz, 1H, H3), 2.45 (t, $\mathrm{J}=2.4 \mathrm{~Hz}, 1 \mathrm{H}, \mathrm{H} 9), 2.37(\mathrm{~s}, 3 \mathrm{H}, \mathrm{SCOCH}$ ), $2.12(\mathrm{~s}$, $\left.3 \mathrm{H}, \mathrm{COCH}_{3}\right), 2.05\left(\mathrm{~s}, 3 \mathrm{H}, \mathrm{COCH}_{3}\right), 2.04\left(\mathrm{~s}, 3 \mathrm{H}, \mathrm{COCH}_{3}\right) .{ }^{13} \mathrm{C} \mathrm{NMR}\left(101 \mathrm{MHz}, \mathrm{CDCl}_{3}\right) \delta_{\mathrm{C}} 192.7\left(\mathrm{SCOCH}_{3}\right), 170.6\left(\mathrm{COCH}_{3}\right), 170.4$ $\left(\mathrm{COCH}_{3}\right), 169.8\left(\mathrm{COCH}_{3}\right), 80.6$ (C1), 79.0 (C8/C9), 76.8 (C3), 75.4 (C5/C8/C9), 75.3 (C5/C8), 67.5 (C2), 65.8 (C4), 61.9 (C6), 56.7 (C7), $31.0\left(\mathrm{SCOCH}_{3}\right), 21.0\left(\mathrm{COCH}_{3}\right), 20.9\left(\mathrm{COCH}_{3}\right), 20.8\left(\mathrm{COCH}_{3}\right)$. HRMS (ES): Calcd. for $\mathrm{C}_{17} \mathrm{H}_{22} \mathrm{O}_{9} \mathrm{SNH}_{4}: 420.1328$; found. 420.1328.

\section{Synthesis of GIcNAc alkyne thioacetate C2}
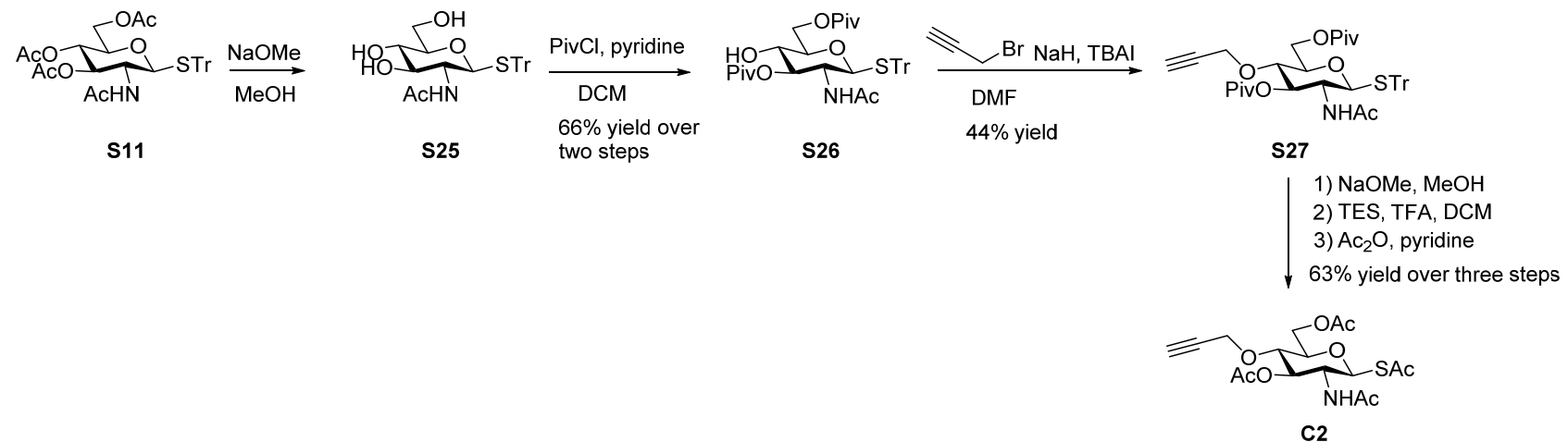

Triphenylmethyl 1-thio-2-acetamido-2-deoxy- $\beta$-D-glucopyranoside S25

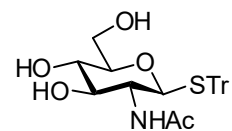

A sodium methoxide solution was prepared by dissolving a catalytic amount of metallic sodium in dry methanol (2 $\mathrm{mL}) \mathrm{under}$ a $\mathrm{N}_{2}$ atm. $1 \mathrm{~mL}$ of the sodium methoxide solution was then added to a solution of the acetylated GlcNAc thiol S11 (5.60 g; $9.25 \mathrm{mmol} ; 1 \mathrm{equiv}$.) dissolved in dry methanol $(40 \mathrm{~mL})$ under a $\mathrm{N}_{2}$ atm. When the reaction had reached completion, methanol was evaporated and the product $\mathbf{S 2 5}$ was used in the following reaction without further purification.

TriphenyImethyl 1-thio-2-acetamido-2-deoxy-3,6-di-O-pivaloyl- $\beta$-D-glucopyranoside S26

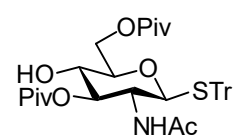

The GlcNAc triol S25 (4.44 g; 9.25 mmol; 1 equiv.) was dissolved in a mixture of dry dichloromethane (16 mL) and dry pyridine (40 mL) under a $\mathrm{N}_{2}$ atm. The mixture was then cooled to $0^{\circ} \mathrm{C}$ before $\mathrm{PivCl}(3.4 \mathrm{~mL} ; 25.9 \mathrm{mmol} ; 2.8$ equiv.) was added dropwise. After 7 hours the reaction had reached completion and was diluted with dichloromethane. The organic phase was washed with $1 \mathrm{M} \mathrm{HCl}$ (aq.) and $\mathrm{H}_{2} \mathrm{O}$, dried over magnesium sulfate, filtered and evaporated. The product $\mathbf{S} 2 \mathbf{6}$ was purified by column chromatography (pentane/ethyl acetate $3: 1$ ) and isolated as white foam in $66 \%$ yield in two steps $\left(3.93 \mathrm{~g} ; 6.06 \mathrm{mmol} \text { ). } R_{\mathrm{f}} 0.70 \text { (ethyl acetate/pentane } 2: 1 \text { ). [ } \alpha\right]_{\mathrm{D}}{ }^{295 \mathrm{~K}}$ $109.4\left(c\right.$ 1, $\left.\mathrm{CHCl}_{3}\right) .{ }^{1} \mathrm{H}$ NMR $\left(400 \mathrm{MHz}, \mathrm{CDCl}_{3}\right) \delta_{\mathrm{H}} 7.45(\mathrm{~d}, \mathrm{~J}=7.2 \mathrm{~Hz}, 6 \mathrm{H}, \mathrm{ArH}), 7.33-7.17(\mathrm{~m}, 9 \mathrm{H}, \operatorname{ArH}), 5.09(\mathrm{~d}, \mathrm{~J}=10.1 \mathrm{~Hz}, 1 \mathrm{H}$, $\mathrm{NH}), 4.52(\mathrm{t}, \mathrm{J}=9.7 \mathrm{~Hz}, 1 \mathrm{H}, \mathrm{H} 3), 4.33-4.14(\mathrm{~m}, 2 \mathrm{H}, \mathrm{H} 2$ and H6a), $3.96(\mathrm{dd}, \mathrm{J}=12.1,2.1 \mathrm{~Hz}, 1 \mathrm{H}, \mathrm{H} 6 \mathrm{~b}), 3.51-3.36(\mathrm{~m}, 2 \mathrm{H}, \mathrm{H} 1 \mathrm{and}$ $\left.\mathrm{H} 4), 2.73(\mathrm{~d}, \mathrm{~J}=9.7 \mathrm{~Hz}, 1 \mathrm{H}, \mathrm{H} 5), 1.85(\mathrm{~s}, 3 \mathrm{H}, \mathrm{NCOCH})_{3}\right), 1.22\left(\mathrm{~s}, 9 \mathrm{H}, \mathrm{OC}\left(\mathrm{CH}_{3}\right)_{3}\right), 1.14\left(\mathrm{~s}, 9 \mathrm{H}, \mathrm{OC}\left(\mathrm{CH}_{3}\right)_{3}\right) .{ }^{13} \mathrm{C} \mathrm{NMR}(101 \mathrm{MHz}, \mathrm{CDCl})_{3}$ $\delta_{\mathrm{C}} 179.7\left(\mathrm{COOC}\left(\mathrm{CH}_{3}\right)_{3}\right), 169.6\left(\mathrm{COOC}\left(\mathrm{CH}_{3}\right)_{3}\right), 144.7,130.1,127.8,126.9(\mathrm{ArC}), 85.0(\mathrm{C} 1), 78.1(\mathrm{C} 5), 76.3(\mathrm{C} 3), 68.6(\mathrm{SCPh}), 68.4$ (C4), $62.8(\mathrm{C} 6), 52.0(\mathrm{C} 2), 39.1\left(\mathrm{OC}\left(\mathrm{CH}_{3}\right)_{3}\right), 39.0\left(\mathrm{OC}\left(\mathrm{CH}_{3}\right)_{3}\right) 27.3\left(\mathrm{OC}\left(\mathrm{CH}_{3}\right)_{3}\right), 27.1\left(\mathrm{OC}\left(\mathrm{CH}_{3}\right)_{3}\right), 23.4\left(\mathrm{NCOCH}_{3}\right)$. HRMS (ES): Calcd. for $\mathrm{C}_{37} \mathrm{H}_{45} \mathrm{NO}_{7} \mathrm{SNa}$ : 670.2814; found. 670.2814 . 


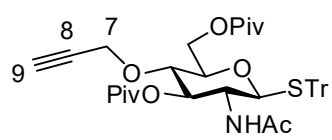

$\mathrm{NaH}, 60 \%$ in mineral oil, $(0.015 \mathrm{~g} ; 0.386 \mathrm{mmol} ; 1.05$ equiv.) was added to a solution of the diPiv-protected GlcNAc thiol S26 (0.238 g; $0.367 \mathrm{mmol} ; 1$ equiv.), propargyl bromide, $80 \%$ in toluene, $(0.16 \mathrm{~mL} ; 1.84 \mathrm{mmol} ; 5$ equiv.) and TBAI (0.014 g; $0.0367 \mathrm{mmol} ; 0.1$ equiv.) dissolved in dry DMF ( $1 \mathrm{~mL}$ ) under a $\mathrm{N}_{2}$ atm. After 1 hour more $\mathrm{NaH}(0.005 \mathrm{~g} ; 0.208 \mathrm{mmol} ; 0.35$ equiv) was added. After four hours the reaction had reached completion and was diluted with ethyl acetate. The organic phase was washed with $\mathrm{H}_{2} \mathrm{O}$ and brine, dried over magnesium sulfate, filtered and evaporated. The product $\mathbf{S} 27$ was purified by column chromatography (pentane/ethyl acetate 5:1) and isolated as clear oil in $44 \%$ yield $(0.112 \mathrm{~g} ; 0.163 \mathrm{mmol})$ ) ). $R_{\mathrm{f}} 0.31$ (pentane/ethyl acetate $\left.2: 1\right)$. [ $\left.\alpha\right]_{D}{ }^{295 K}-62.0\left(c 1, \mathrm{CHCl}_{3}\right) .{ }^{1} \mathrm{H} \mathrm{NMR}(400$ $\left.\mathrm{MHz}, \mathrm{CDCl}_{3}\right) \delta_{\mathrm{H}} 7.44(\mathrm{~d}, \mathrm{~J}=7.1 \mathrm{~Hz}, 6 \mathrm{H}, \mathrm{ArH}), 7.33-7.17(\mathrm{~m}, 9 \mathrm{H}, \mathrm{ArH}), 4.93(\mathrm{~d}, \mathrm{~J}=10.4 \mathrm{~Hz}, 1 \mathrm{H}, \mathrm{NH}), 4.62(\mathrm{dd}, \mathrm{J}=10.4,9.3 \mathrm{~Hz}, 1 \mathrm{H}$, $\mathrm{H} 3$ ), 4.27 (q, $\mathrm{J}=10.4 \mathrm{~Hz}, 1 \mathrm{H}, \mathrm{H} 2), 4.22-4.07$ (m, 3H, H6a and H7), 4.00 (dd, J = 12.2, 3.2 Hz, 1H, H6b), $3.62(\mathrm{t}, \mathrm{J}=9.3 \mathrm{~Hz}, 1 \mathrm{H}, \mathrm{H} 4)$, $3.32(\mathrm{~d}, \mathrm{~J}=10.4 \mathrm{~Hz}, 1 \mathrm{H}, \mathrm{H} 1), 2.78(\mathrm{~d}, \mathrm{~J}=9.3 \mathrm{~Hz}, 1 \mathrm{H}, \mathrm{H} 5), 2.42(\mathrm{t}, \mathrm{J}=2.4 \mathrm{~Hz}, 1 \mathrm{H}, \mathrm{H} 9), 1.86\left(\mathrm{~s}, 3 \mathrm{H}, \mathrm{NCOCH}_{3}\right), 1.25\left(\mathrm{~s}, 9 \mathrm{H}, \mathrm{OC}\left(\mathrm{CH}_{3}\right)_{3}\right)$, $1.18\left(\mathrm{~s}, 9 \mathrm{H}, \mathrm{OC}\left(\mathrm{CH}_{3}\right)_{3}\right) .{ }^{13} \mathrm{C} \mathrm{NMR}\left(101 \mathrm{MHz}, \mathrm{CDCl}_{3}\right) \delta_{\mathrm{C}} 179.0\left(\mathrm{COOC}\left(\mathrm{CH}_{3}\right)_{3}\right), 178.2\left(\mathrm{COOC}\left(\mathrm{CH}_{3}\right)_{3}\right), 169.5\left(\mathrm{NCOCH}_{3}\right), 144.6,130.1$, 127.8, 127.0 (ArC), 85.1 (C1), 78.8 (C8/C9), 76.7 (C5), 76.2 (C3), 75.5 (C4), 74.9 (C9/C8), 68.7 (SCPh $), 62.5$ (C6), 59.7 (C7), 52.4 (C2), $39.1\left(\mathrm{OC}\left(\mathrm{CH}_{3}\right)_{3}\right), 39.0\left(\mathrm{OC}\left(\mathrm{CH}_{3}\right)_{3}\right), 27.4\left(\mathrm{OC}\left(\mathrm{CH}_{3}\right)_{3}\right), 27.2\left(\mathrm{OC}\left(\mathrm{CH}_{3}\right)_{3}\right), 23.5\left(\mathrm{NHCOCH}_{3}\right)$. HRMS (ES): Calcd. for $\mathrm{C}_{40} \mathrm{H}_{47} \mathrm{NO}_{7} \mathrm{SNa}$ : 708.2971; found. 708.2967.

\section{2-Acetamido-1-S-acetyl-2-deoxy-3,6-di-O-acetyl-4-O-propargyl- $\beta$-D-1-thio-glucopyranose C2}

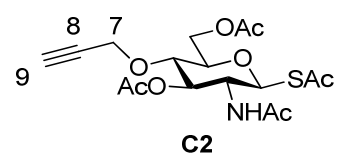

NaOMe (25\% in methanol, $0.51 \mathrm{~mL}, 3$ equiv) was added to a solution of the alkylated compound $\mathbf{S} 27$ ( $0.508 \mathrm{~g} ; 0.741 \mathrm{mmol} ; 1 \mathrm{equiv}$.) dissolved in dry methanol $(7 \mathrm{~mL})$ under a $\mathrm{N}_{2}$ atm. The mixture was then heated to $40{ }^{\circ} \mathrm{C}$ overnight. Once the reaction had run to completion the solvent was evaporated and the residue was re-dissolved in dry dichloromethane (16 $\mathrm{mL}) \mathrm{under}$ a $\mathrm{N}_{2}$ atm. To this solution triethylsilane $(0.14 \mathrm{~mL} ; 1.71 \mathrm{mmol} ; 2.3$ equiv) and TFA $(7 \mathrm{~mL})$ were added. After two hours the reaction had run to completion and the solvents were evaporated. Co-evaporation with toluene was performed three times before the residue was re-dissolved in dry pyridine $(3 \mathrm{~mL})$ and acetic anhydride $(3 \mathrm{~mL})$ under a $\mathrm{N}_{2}$ atm. and left overnight. Once the acetylation had reached completion the reaction carefully quenched by the addition of $\mathrm{H}_{2} \mathrm{O}$. The reaction mixture was left for 20 minutes before it was diluted with ethyl acetate. The organic phase was washed with $1 \mathrm{M} \mathrm{HCl}(\mathrm{aq}), \mathrm{H}_{2} \mathrm{O}$, sat $\mathrm{NaHCO}_{3}(\mathrm{aq})$ and brine, dried over magnesium sulfate, filtered and evaporated. The product was purified by column chromatography (ethyl acetate/pentane 2:1) and isolated as a light yellow, amorphous solid in $63 \%$ yield over three steps $(0.186 \mathrm{~g} ; 0.463 \mathrm{mmol}) . R_{\mathrm{f}} 0.24$ (ethyl acetate/pentane 2:1). $[\alpha]_{\mathrm{D}}{ }^{295 \mathrm{~K}}+14.8\left(\mathrm{c} 1, \mathrm{CHCl}_{3}\right) .{ }^{1} \mathrm{H} \mathrm{NMR}$ $\left(400 \mathrm{MHz}, \mathrm{CDCl}_{3}\right) \delta_{\mathrm{H}} 6.37(\mathrm{~d}, \mathrm{~J}=10.0 \mathrm{~Hz}, 1 \mathrm{H}, \mathrm{NH}), 5.10$ (dd, J = 16.8, $10.2 \mathrm{~Hz}, 2 \mathrm{H}, \mathrm{H} 1$ and H3), $4.38-4.07$ (m, 5H, H2, H6 and H7), $3.72-3.58(\mathrm{~m}, 2 \mathrm{H}, \mathrm{H} 4$ and H5), 2.47 (s, 1H, H9), 2.30 (s, 3H, sCOCH $), 2.06\left(\mathrm{~s}, 3 \mathrm{H}, \mathrm{COCH}_{3}\right), 2.02\left(\mathrm{~s}, 3 \mathrm{H}, \mathrm{COCH}_{3}\right), 1.86(\mathrm{~s}, 3 \mathrm{H}$, $\left.\mathrm{NCOCH}_{3}\right) .{ }^{13} \mathrm{C}$ NMR $\left(101 \mathrm{MHz}, \mathrm{CDCl}_{3}\right) \delta_{\mathrm{C}} 193.6\left(\mathrm{SCOCH}_{3}\right), 171.4,170.7,170.3\left(\mathrm{NCOCH}_{3}\right.$ and $\left.\mathrm{COCH}_{3}\right), 81.2(\mathrm{C} 1), 78.9(\mathrm{C} / \mathrm{C} 9), 76.9$ (C4/C5), 76.5 (C3), 75.4 (C9/C8), 74.9 (C5/C4), 62.8 (C6), 59.7 (C7), $51.9(\mathrm{C} 2), 30.8\left(\mathrm{SCOCH}_{3}\right), 23.1\left(\mathrm{NCOCH}_{3}\right), 21.1\left(\mathrm{COCH}_{3}\right), 20.9$ $\left(\mathrm{COCH}_{3}\right)$. HRMS (ES): Calcd. for $\mathrm{C}_{17} \mathrm{H}_{24} \mathrm{NO}_{8} \mathrm{SH}: 402.1223$; found. 402.1225.

\section{Synthesis of manno alkyne thioacetate C3}

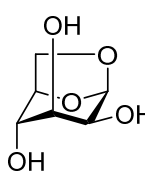

S6

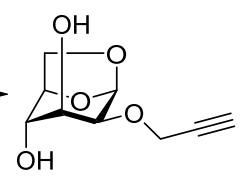

S28

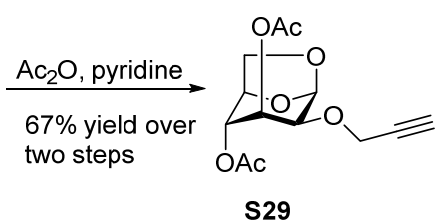

S29

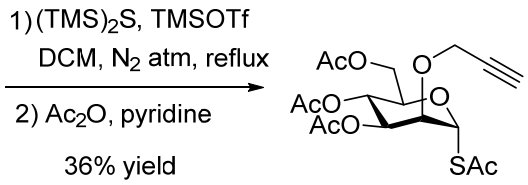

C3 


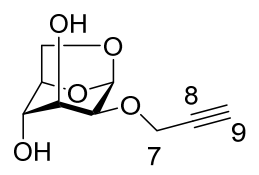

$\mathrm{Bu}_{2} \mathrm{SnO}$ (0.690 g; $2.75 \mathrm{mmol} ; 1.1$ equiv.) was added to a solution of mannosan $\mathbf{S 6}$ (0.406 g; $2.46 \mathrm{mmol} ; 1$ equiv.) dissolved in dry benzene $(10 \mathrm{~mL})$ under a $\mathrm{N}_{2}$ atm. The mixture was heated to reflux for two hours, after which TBAl $(0.925 \mathrm{~g} ; 2.50 \mathrm{mmol} ; 1 \mathrm{equiv}$.) and propargyl bromide ( $80 \%$ in toluene, $0.67 \mathrm{~mL} ; 7.51 \mathrm{mmol} ; 3$ equiv.) was added and the mixture was heated to reflux overnight. When the reaction had reached completion it was cooled to rt, and benzene was evaporated. The crude product $\mathbf{S} 28$ was acetylated without further purification.

\section{1,6-anhydro-3,4-O-acetyl-2-O-propargyl- $\beta$-D-mannopyranose S29}

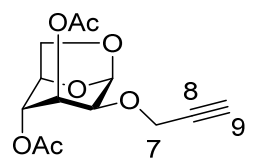

The residue $\mathbf{S} 28$ was dissolved in dry pyridine $(3 \mathrm{~mL})$ and acetic anhydride $(3 \mathrm{~mL})$ under a $\mathrm{N}_{2}$ atm. and left overnight. When the reaction had reached completion it was carefully quenched by the addition of $\mathrm{H}_{2} \mathrm{O}$ and left for 20 minutes before it was diluted with ethyl acetate. The organic phase was washed with $1 \mathrm{M} \mathrm{HCl}(\mathrm{aq}), \mathrm{H}_{2} \mathrm{O}$, sat $\mathrm{NaHCO}_{3}(\mathrm{aq})$ and brine, dried over magnesium sulfate, filtered and evaporated. The product $\mathbf{S} 29$ was purified by column chromatography (dichloromethane/ethyl acetate $50: 1$ ) and isolated as clear oil in $67 \%$ yield over two steps $(0.476 \mathrm{~g} ; 1.67 \mathrm{mmol}) . R_{\mathrm{f}} 0.49$ (pentane ethyl acetate 1:1). $[\alpha]_{\mathrm{D}}{ }^{295 \mathrm{~K}}-42.4\left(c 1, \mathrm{CHCl}_{3}\right) .{ }^{1} \mathrm{H} \mathrm{NMR}(400 \mathrm{MHz}$, $\left.\mathrm{CDCl}_{3}\right) \delta_{\mathrm{H}} 5.50(\mathrm{~s}, 1 \mathrm{H}, \mathrm{H} 1), 5.30$ (ddd, $\left.\mathrm{J}=5.2,3.0,1.5 \mathrm{~Hz}, 1 \mathrm{H}, \mathrm{H} 3\right), 4.79(\mathrm{~s}, 1 \mathrm{H}, \mathrm{H} 4), 4.59$ (d, J = 4.9 Hz, 1H, H5), $4.29-4.10(\mathrm{~m}, 3 \mathrm{H}$, $\mathrm{H} 6 \mathrm{a}$ and $\mathrm{H} 7$ ), $3.88-3.78(\mathrm{~m}, 2 \mathrm{H}, \mathrm{H} 2$ and $\mathrm{H} 6 \mathrm{~b}), 2.46(\mathrm{t}, \mathrm{J}=2.4 \mathrm{~Hz}, 1 \mathrm{H}, \mathrm{H} 9), 2.16\left(\mathrm{~s}, 3 \mathrm{H}, \mathrm{COCH}_{3}\right), 2.15\left(\mathrm{~s}, 3 \mathrm{H}, \mathrm{COCH}_{3}\right) .{ }^{13} \mathrm{C} \mathrm{NMR}(101$ $\left.\mathrm{MHz}, \mathrm{CDCl}_{3}\right) \delta_{\mathrm{C}} 170.0\left(\mathrm{COCH}_{3}\right), 169.9\left(\mathrm{COCH}_{3}\right), 100.6$ (C1), 79.2 (C8/C9), 75.5 (C9/C8), 73.8 (C5), $72.2,72.0$ (C2 and C4), 67.5 (C3), 65.3 (C6), $57.6(\mathrm{C} 7), 21.1\left(\mathrm{COCH}_{3}\right), 21.1\left(\mathrm{COCH}_{3}\right)$. HRMS (ES): Calcd. for $\mathrm{C}_{13} \mathrm{H}_{16} \mathrm{O}_{7} \mathrm{Na}$ : 307.0794; found. 307.0795.

\section{1-S-Acetyl-3,4,6-tri-O-acetyl-2-O-propargy-1-thio- $\alpha$-D-mannopyranose C3}

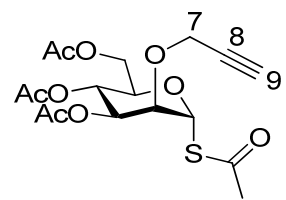

TMSOTf (0.075 mL; $0.411 \mathrm{mmol} ; 1$ equiv.) was added dropwise to a solution of protected alkylated mannosan S29 (0.119 g; 0.411 mmol; 1 equiv.) and (TMS) $)_{2} \mathrm{~S}\left(0.17 \mathrm{~mL} ; 0.822 \mathrm{mmol} ; 2\right.$ equiv.) dissolved in dry dichloromethane $(7 \mathrm{~mL})$ under a $\mathrm{N}_{2}$ atm. The mixture was heated to reflux and left for three days at reflux. More (TMS $)_{2} \mathrm{~S}(0.17 \mathrm{~mL} ; 0.822 \mathrm{mmol} ; 2$ equiv. $)$ was added and the reaction was left for three days at $\mathrm{rt}$. When the reaction had run to completion, dry pyridine $(1 \mathrm{~mL})$ and acetic anhydride $(1 \mathrm{~mL})$ were added under a $\mathrm{N}_{2}$ atm. The acetylation reaction was left overnight. When the reaction had run to completion it was quenched by dropwise addition of $\mathrm{H}_{2} \mathrm{O}$. After 20 min the mixture was diluted with ethyl acetate and the organic phase was washed with $1 \mathrm{M} \mathrm{HCl}(\mathrm{aq}), \mathrm{H}_{2} \mathrm{O}, \mathrm{sat} \mathrm{NaHCO} 3$ and brine sequentially. The organic phase was then dried over magnesium sulfate, filtered and evaporated. The product $\mathbf{C} 3$ was purified by column chromatography (dichloromethane/ethyl acetate 3:0.1) and obtained as light yellow oil in $36 \%$ yield $(0.060 \mathrm{~g} ; 0.148 \mathrm{mmol})$. $R_{\mathrm{f}} 0.46$ (pentane/ethyl acetate 1:1). $[\alpha]_{\mathrm{D}}{ }^{295 \mathrm{~K}}+26.4\left(\mathrm{c} 1, \mathrm{CHCl}_{3}\right) .{ }^{1} \mathrm{H} \mathrm{NMR}\left(400 \mathrm{MHz}, \mathrm{CDCl}_{3}\right) \delta_{\mathrm{H}} 6.07(\mathrm{~d}, \mathrm{~J}=3.2 \mathrm{~Hz}, 1 \mathrm{H}, \mathrm{H} 1), 5.38(\mathrm{t}, \mathrm{J}=$ $10.1 \mathrm{~Hz}, 1 \mathrm{H}, \mathrm{H} 4$ ), 5.02 (dd, J = 10.1, 3.2 Hz, 1H, H3), 4.35 (dd, J = 4.5, $2.4 \mathrm{~Hz}, 2 \mathrm{H}, \mathrm{H} 7$ ), $4.31-4.23$ (m, 1H, H6a), 4.12 (dd, J = 3.2, $2.0 \mathrm{~Hz}, 1 \mathrm{H}, \mathrm{H} 2$ ), 4.04 (dd, J = 12.4, $2.3 \mathrm{~Hz}, 1 \mathrm{H}, \mathrm{H6b}$ ), 3.83 (ddd, J = 10.1, 4.5, $2.3 \mathrm{~Hz}, 1 \mathrm{H}, \mathrm{H} 5), 2.42$ (s, 3H, SCOCH $), 2.16$ (s, 1H, H9), $2.07\left(\mathrm{~s}, 6 \mathrm{H}, 2 \mathrm{COCH}_{3}\right), 2.03\left(\mathrm{~s}, 3 \mathrm{H}, \mathrm{COCH}_{3}\right) .{ }^{13} \mathrm{C} \mathrm{NMR}\left(101 \mathrm{MHz}, \mathrm{CDCl}_{3}\right) \delta_{\mathrm{C}} 191.5\left(\mathrm{SCOCH}_{3}\right), 170.9\left(\mathrm{COCH}_{3}\right), 170.4\left(\mathrm{COCH}_{3}\right), 169.5$ $\left(\mathrm{COCH}_{3}\right), 79.7$ (C1), 78.8 (C8/C9), 76.3 (C2), 75.6 (C9/C8), 73.0 (C5), 71.4 (C3), 65.7 (C4), 62.3 (C6), 57.8 (C7), $31.5(\mathrm{SCOCH}), 21.0$ $\left(\mathrm{COCH}_{3}\right), 20.9\left(\mathrm{COCH}_{3}\right), 20.8\left(\mathrm{COCH}_{3}\right)$. HRMS (ES): Calcd. for $\mathrm{C}_{17} \mathrm{H}_{22} \mathrm{O}_{9} \mathrm{SNH}_{4}: 420.1328$; found. 420.1338. 


\section{Synthesis of Group D building blocks}

\section{Synthesis of galacto alkyne D1}

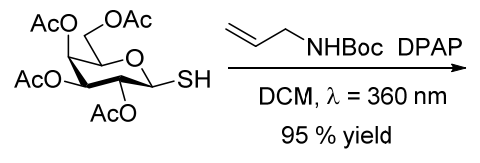

A2

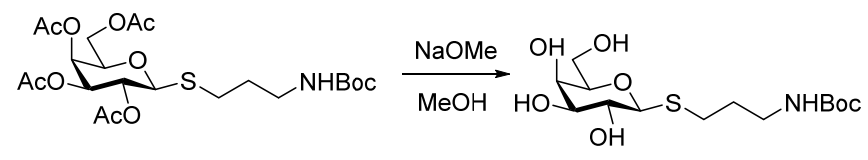

S30

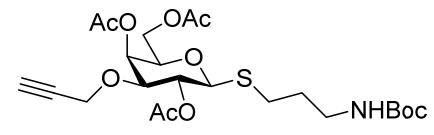

D1

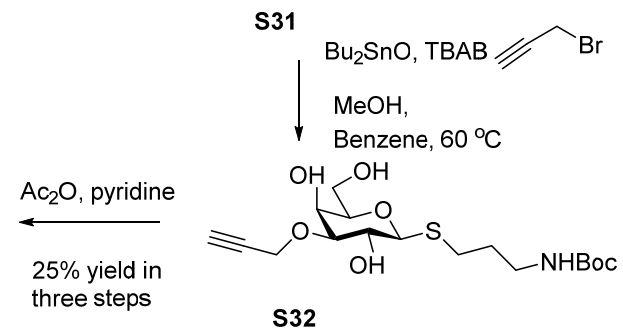

\section{tButyl allylcarbamate}

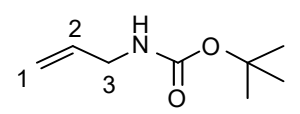

Allyl amine (6.55 mL; $87.6 \mathrm{mmol} ; 1$ equiv.) and triethyl amine (27 mL; $193 \mathrm{mmol} ; 2.2$ equiv.) were dissolved in dry dichloromethane $(100 \mathrm{~mL})$ under a $\mathrm{N}_{2} \mathrm{~atm}$. The solution was then cooled to $0^{\circ} \mathrm{C}$ before $\mathrm{Boc}_{2} \mathrm{O}(21.0 \mathrm{~g} ; 96.3 \mathrm{mmol} ; 1.1$ equiv.) was added in two portions. The reaction was allowed to warm to rt and left overnight. When the reaction had run to completion the solvent was evaporated and the residue was purified by column chromatography (pentane/ethyl acetate 100:2). The product was obtained as a clear solid in $92 \%$ yield (12.7 g; $81.0 \mathrm{mmol}$ ). $R_{\mathrm{f}} 0.31$ (cyclohexane/ethyl acetate $\left.5: 1\right)$. ${ }^{1} \mathrm{H}$ NMR $\left(400 \mathrm{MHz}, \mathrm{CDCl}_{3}\right) \delta_{\mathrm{H}} 5.84(\mathrm{~m}, 1 \mathrm{H}, \mathrm{H} 2), 5.14(\mathrm{ddd}, \mathrm{J}=13.7$, $11.1,1.5 \mathrm{~Hz}, 2 \mathrm{H}, \mathrm{H} 1), 4.66$ (br. s, $\left.1 \mathrm{H}, \mathrm{NH}), 3.75(\mathrm{~s}, 2 \mathrm{H}, \mathrm{H} 3), 1.45\left(\mathrm{~s}, 9 \mathrm{H}, 3 \mathrm{CH}_{3}\right) .{ }^{13} \mathrm{C} \mathrm{NMR}\left(101 \mathrm{MHz}, \mathrm{CDCl}_{3}\right) \delta_{\mathrm{C}} 155.9\left(\mathrm{COOC}_{(\mathrm{CH}}\right)_{3}\right)$, 135.0 (C2), 115.8 (C1), $79.5\left(\mathrm{OC}\left(\mathrm{CH}_{3}\right)_{3}\right), 43.2(\mathrm{C} 3), 28.5\left(\mathrm{CH}_{3}\right)$. HRMS (ES): Calcd. for $\mathrm{C}_{8} \mathrm{H}_{15} \mathrm{NO}_{2} \mathrm{Na}$ : 180.1000; found. 180.0996. NMR data are in accordance with literature-values. ${ }^{20}$

\section{(tButyl(3-mercaptopropyl)carbamate) 2,3,4,6-tetra-O-acetyl- $\beta$-D-galactopyranoside S30}

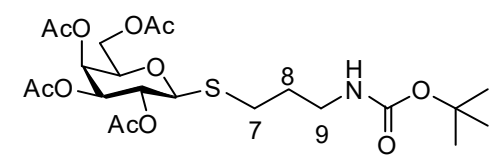

The per-acetylated galactosyl thiol $\mathbf{A} 2$ (0.598 g; $1.64 \mathrm{mmol} ; 1$ equiv.), the Boc protected allyl amine (0.310 g; $1.97 \mathrm{mmol} ; 1.2 \mathrm{equiv}$.) and DPAP (0.042 g; $0.164 \mathrm{mmol} ; 0.1$ equiv.) were dissolved in dichloromethane $(6 \mathrm{~mL})$ and irradiated at $360 \mathrm{~nm}$ for 4 hours. The solvent was then evaporated, and the residue was purified by column chromatography (pentane/ethyl acetate 4:1). The product S30

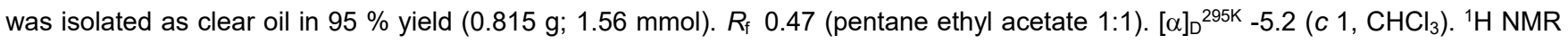
$\left(400 \mathrm{MHz}, \mathrm{CDCl}_{3}\right) \delta_{\mathrm{H}} 5.42$ (dd, J = 3.4, $\left.0.9 \mathrm{~Hz}, 1 \mathrm{H}, \mathrm{H} 4\right), 5.23$ (t, J=10.0 Hz, 1H, H2), 5.03 (dd, J = 10.0, 3.4 Hz, 1H, H3), $4.73(\mathrm{br} . \mathrm{s}$, $1 \mathrm{H}, \mathrm{NH}), 4.46(\mathrm{~d}, \mathrm{~J}=10.0 \mathrm{~Hz}, 1 \mathrm{H}, \mathrm{H} 1), 4.22-4.05$ (m, 2H, H6), $3.93(\mathrm{td}, \mathrm{J}=6.6,0.9 \mathrm{~Hz}, 1 \mathrm{H}, \mathrm{H} 5), 3.22(\mathrm{~d}, \mathrm{~J}=5.8 \mathrm{~Hz}, 2 \mathrm{H}, \mathrm{H} 9), 2.82-$ $2.63(\mathrm{~m}, 2 \mathrm{H}, \mathrm{H} 7), 2.15\left(\mathrm{~s}, 3 \mathrm{H}, \mathrm{COCH}_{3}\right), 2.06\left(\mathrm{~s}, 3 \mathrm{H}, \mathrm{COCH}_{3}\right), 2.04\left(\mathrm{~s}, 3 \mathrm{H}, \mathrm{COCH}_{3}\right), 1.98\left(\mathrm{~s}, 3 \mathrm{H}, \mathrm{COCH}_{3}\right), 1.79$ (quint, J = 6.7 Hz, 2H, $\mathrm{H} 8), 1.43\left(\mathrm{~s}, 9 \mathrm{H}, \mathrm{OC}\left(\mathrm{CH}_{3}\right)_{3}\right) .{ }^{13} \mathrm{C}$ NMR $\left(101 \mathrm{MHz}, \mathrm{CDCl}_{3}\right) \delta_{\mathrm{C}} 170.5\left(\mathrm{COCH}_{3}\right), 170.3\left(\mathrm{COCH}_{3}\right), 170.2\left(\mathrm{COCH}_{3}\right), 169.8\left(\mathrm{COCH}_{3}\right), 156.1$ ( $\mathrm{NHCOOC}), 84.2(\mathrm{C} 1), 79.4\left(\mathrm{OC}\left(\mathrm{CH}_{3}\right)_{3}\right), 74.7$ (C5), $71.9(\mathrm{C} 3), 67.4(\mathrm{C} 4), 67.2(\mathrm{C} 2), 61.6(\mathrm{C} 6), 39.2(\mathrm{C} 9), 29.9(\mathrm{C} 8), 28.5\left(\mathrm{OC}(\mathrm{CH})_{3}\right)$, $27.4(\mathrm{C} 7), 21.0\left(\mathrm{COCH}_{3}\right), 20.8\left(\mathrm{COCH}_{3}\right), 20.8\left(\mathrm{COCH}_{3}\right), 20.7\left(\mathrm{COCH}_{3}\right)$. HRMS (ES): Calcd. for $\mathrm{C}_{22} \mathrm{H}_{35} \mathrm{NO}_{11} \mathrm{SNa}$ : 544.1829; found. 544.1827.

(tButyl(3-mercaptopropyl)carbamate)- $\beta$-D-galactopyranoside S31 


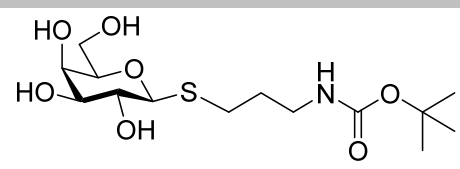

The per-acetylated thioether $\mathbf{S} 30$ (3.35 g; $6.42 \mathrm{mmol}$; 1 equiv.) was dissolved in dry methanol (20 mL) under a $\mathrm{N}_{2}$ atm, before $\mathrm{NaOMe}$ ( $25 \%$ in methanol, $0.3 \mathrm{~mL}$ ) was added. When the reaction had reached completion, the solvent was evaporated and the residue was used without further purification.

\section{(tButyl(3-mercaptopropyl)carbamate)-3-O-propargyl- $\beta$-D-galactopyranoside S32}

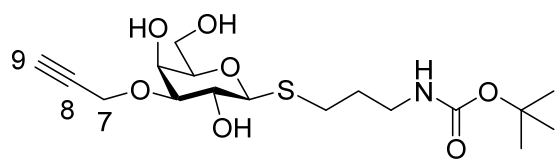

$\mathrm{Bu}_{2} \mathrm{SnO}$ (0.607 g; $2.42 \mathrm{mmol} ; 1.1$ equiv.) was added to a solution of deprotected thioether $\mathbf{S} 31$ ( $0.776 \mathrm{~g} ; 2.20 \mathrm{mmol} ; 1$ equiv.) dissolved in dry methanol $(7 \mathrm{~mL})$ under a $\mathrm{N}_{2}$ atm. The mixture was heated to reflux for three hours, after which the methanol was evaporated, and the residue was re-dissolved in dry benzene $(10 \mathrm{~mL})$ under a $\mathrm{N}_{2}$ atm. TBAB (0.708 g; $2.20 \mathrm{mmol} ; 1$ equiv.) and propargyl bromide ( $80 \%$ in toluene, $0.59 \mathrm{~mL} ; 6.59 \mathrm{mmol} ; 3$ equiv.) was added and the mixture was heated to $60^{\circ} \mathrm{C}$ overnight. When the reaction had run to completion it was cooled to $\mathrm{rt}$, and benzene was evaporated. The residue was purified by column chromatography (dichloromethane/methanol 100:1 to $100: 2.5$ ) and isolated as yellow oil. Even though it was necessary to perform column chromatography at this point to remove an impurity that after acetylation was co-polar with the desired product, the alkylated product S31 was not pure, and therefore the product was not fully characterized at this stage. This was instead done after acetylation to obtain the acetylated product D1.

\section{(tButyl(3-mercaptopropyl)carbamate)-2,4,6-tri-O-acetyl-3-O-propargyl- $\beta$-D-galactopyranoside D1}

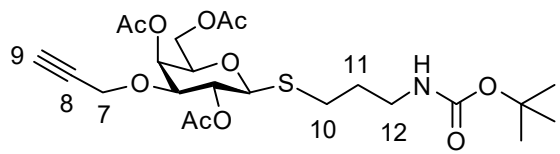

The product $\mathbf{S} 32$ ( $0.332 \mathrm{~g} ; 0.849 \mathrm{mmol} ; 1$ equiv.) was dissolved in dry pyridine (2 mL) and acetic acid (2 mL) under a $\mathrm{N}_{2}$ atm. and left overnight. When the reaction had reached completion it was carefully quenched by the addition of $\mathrm{H}_{2} \mathrm{O}$. The reaction mixture was left for 20 minutes before it was diluted with ethyl acetate. The organic phase was washed with $1 \mathrm{M} \mathrm{HCl}(\mathrm{aq}), \mathrm{H}_{2} \mathrm{O}, \mathrm{sat} \mathrm{NaHCO}_{3}(\mathrm{aq})$ and brine, dried over magnesium sulfate, filtered and evaporated. The product D1 was isolated as clear oil in $25 \%$ yield in three steps (0.425 g; $0.874 \mathrm{mmol}$ ). $R_{\mathrm{f}} 0.59$ (pentane ethyl acetate 1:1). $[\alpha]_{\mathrm{D}}{ }^{295 \mathrm{~K}}+8.2\left(\mathrm{c} 1, \mathrm{CHCl}_{3}\right) .{ }^{1} \mathrm{H} \mathrm{NMR}\left(400 \mathrm{MHz}, \mathrm{CDCl}_{3}\right) \delta_{\mathrm{H}} 5.41(\mathrm{~d}, \mathrm{~J}=3.1 \mathrm{~Hz}, 1 \mathrm{H}$, $\mathrm{H} 4), 5.06$ (t, J = 9.8 Hz, 1H, H2), 4.77 (br. s, 1H, NH), 4.42 (d, J = 9.8 Hz, 1H, H1), 4.16 (d, J = 2.4 Hz, 2H, H7), 4.11 (d, J=6.7 Hz, 2H, H6), $3.84(\mathrm{t}, \mathrm{J}=6.7 \mathrm{~Hz}, 1 \mathrm{H}, \mathrm{H} 5), 3.80(\mathrm{dd}, \mathrm{J}=9.8,3.1 \mathrm{~Hz}, 1 \mathrm{H}, \mathrm{H} 3), 3.19(\mathrm{br} \mathrm{s}, 2 \mathrm{H}, \mathrm{H} 12), 2.82-2.58(\mathrm{~m}, 2 \mathrm{H}, \mathrm{H} 10), 2.43(\mathrm{t}, \mathrm{J}=2.3 \mathrm{~Hz}$, $1 \mathrm{H}, \mathrm{H} 9), 2.11\left(\mathrm{~s}, 3 \mathrm{H}, \mathrm{COCH}_{3}\right), 2.08\left(\mathrm{~s}, 3 \mathrm{H}, \mathrm{COCH}_{3}\right), 2.04\left(\mathrm{~s}, 3 \mathrm{H}, \mathrm{COCH}_{3}\right), 1.77$ (quint, J = 6.8 Hz, 2H, H11), $1.40\left(\mathrm{~s}, 9 \mathrm{H}, \mathrm{C}\left(\mathrm{CH}_{3}\right)_{3}\right) .{ }^{13} \mathrm{C}$ $\operatorname{NMR}\left(101 \mathrm{MHz}, \mathrm{CDCl}_{3}\right) \delta_{\mathrm{C}} 170.6\left(\mathrm{COCH}_{3}\right), 170.5\left(\mathrm{COCH}_{3}\right), 169.9\left(\mathrm{COCH}_{3}\right), 156.0(\mathrm{NHCOOC}), 83.9(\mathrm{C} 1), 79.3\left(\mathrm{C}\left(\mathrm{CH}_{3}\right)_{3}\right), 79.1(\mathrm{C} 8 / \mathrm{C} 9)$, 76.7 (C3), 75.2 (C5), 74.8 (C9/C8), 68.3 (C2), 65.9 (C4), 62.1 (C6), 56.6 (C7), 39.1 (C12), 29.8 (C11), $28.5\left(C\left(\mathrm{CH}_{3}\right)_{3}\right), 27.1$ (C10), 21.1 $\left(\mathrm{COCH}_{3}\right), 20.9\left(\mathrm{COCH}_{3}\right), 20.8\left(\mathrm{COCH}_{3}\right)$. HRMS (ES): Calcd. for $\mathrm{C}_{23} \mathrm{H}_{35} \mathrm{NO}_{10} \mathrm{SH}$ : 518.2060; found. 518.2059.

\section{Synthesis of galacto alkene D2}
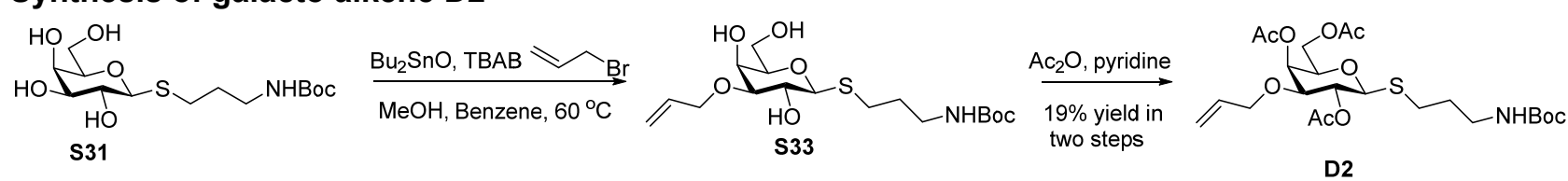

(tButyl(3mercaptopropyl)carbamate)-3-O-allyl- $\beta$-D-galactopyranoside S33 


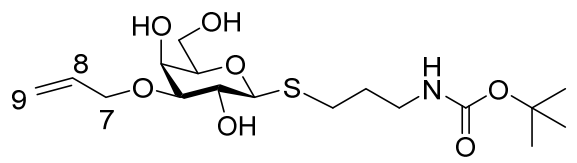

$\mathrm{Bu}_{2} \mathrm{SnO}$ (0.886 g; $3.56 \mathrm{mmol} ; 1.1$ equiv.) was added to a solution of de-protected thioether S31(1.14 g; 3.24 mmol; 1 equiv.) dissolved in dry methanol $(10 \mathrm{~mL})$ under a $\mathrm{N}_{2}$ atm. The mixture was heated to reflux for three hours, after which the methanol was evaporated, and the residue was re-dissolved in dry benzene ( $14 \mathrm{~mL})$ under a $\mathrm{N}_{2}$ atm. TBAB (0.1.05 g; $3.24 \mathrm{mmol} ; 1$ equiv.) and allyl bromide (0.82 $\mathrm{mL} ; 9.71 \mathrm{mmol} ; 3$ equiv.) was added and the mixture was heated to $60^{\circ} \mathrm{C}$ overnight. When the reaction had reached completion it was cooled to rt, and benzene was evaporated. The residue was purified by column chromatography (dichloromethane/methanol $100: 1$ to 100:2.5) and isolated as yellow oil. Even though it was necessary to perform column chromatography at this point to remove an impurity that after acetylation was co-polar with the desired product, the allylated product $\mathbf{S} 3 \mathbf{3}$ was not pure, and therefore the product was not fully characterized at this stage. This was instead done after acetylation to obtain the acetylated product D2.

\section{1-(tButyl(3-mercaptopropyl)carbamate)-2,4,6-tri-O-acetyl-3-O-allyl- $\beta$-D-galactopyranoside D2}

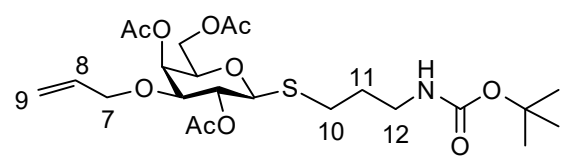

The product S33 (0.331 g; $0.840 \mathrm{mmol} ; 1$ equiv.) was dissolved in dry pyridine ( $2 \mathrm{~mL})$ and acetic acid (2 mL) under a $\mathrm{N}_{2}$ atm. and left overnight. When the reaction had reached completion it was carefully quenched by the addition of $\mathrm{H}_{2} \mathrm{O}$. The reaction mixture was left for 20 minutes before it was diluted with ethyl acetate. The organic phase was washed with $1 \mathrm{M} \mathrm{HCl}(\mathrm{aq}), \mathrm{H}_{2} \mathrm{O}, \mathrm{sat} \mathrm{NaHCO}_{3}(\mathrm{aq})$ and brine, dried over magnesium sulfate, filtered and evaporated. The product $\mathbf{D} 2$ was isolated as clear oil in $19 \%$ yield in two steps $(0.322$ g; $0.619 \mathrm{mmol}$ ). $R_{\mathrm{f}} 0.65$ (pentane ethyl acetate 1:1). $[\alpha]_{\mathrm{D}}{ }^{295 \mathrm{~K}}+10.0\left(\mathrm{c} 1, \mathrm{CHCl}_{3}\right) .{ }^{1} \mathrm{H} \mathrm{NMR}\left(400 \mathrm{MHz}, \mathrm{CDCl}_{3}\right) \delta_{\mathrm{H}} 5.81-5.63(\mathrm{~m}, 1 \mathrm{H}, \mathrm{H} 8)$, $5.40(\mathrm{~d}, \mathrm{~J}=3.1 \mathrm{~Hz}, 1 \mathrm{H}, \mathrm{H} 4), 5.14$ (ddd, $\mathrm{J}=13.8,11.8,1.5 \mathrm{~Hz}, 2 \mathrm{H}, \mathrm{H} 9), 5.05$ (t, J = 9.9 Hz, 1H, H2), 4.81 (br. s, $1 \mathrm{H}, \mathrm{NH}), 4.35(\mathrm{~d}, \mathrm{~J}=$ $9.9 \mathrm{~Hz}, 1 \mathrm{H}, \mathrm{H} 1), 4.14-4.01(\mathrm{~m}, 3 \mathrm{H}, \mathrm{H} 6 \mathrm{a}$ and H7), 3.86 (dd, J = 13.1, 6.3 Hz, 1H, H6b), $3.79(\mathrm{t}, \mathrm{J}=6.3 \mathrm{~Hz}, 1 \mathrm{H}, \mathrm{H} 5), 3.49$ (dd, J = 9.9, $3.1 \mathrm{~Hz}, 1 \mathrm{H}, \mathrm{H} 3$ ), 3.15 (br. s, 2H, H12), $2.82-2.50$ (m, 2H, H10), 2.09 (s, 3H, COCH $), 2.03\left(\mathrm{~s}, 3 \mathrm{H}, \mathrm{COCH}_{3}\right), 2.00\left(\mathrm{~s}, 3 \mathrm{H}, \mathrm{COCH}_{3}\right), 1.73$ (quint, $J=6.7 \mathrm{~Hz}, 2 \mathrm{H}, \mathrm{H} 11), 1.37\left(\mathrm{~s}, 9 \mathrm{H}, \mathrm{C}\left(\mathrm{CH}_{3}\right)_{3}\right) .{ }^{13} \mathrm{C} \mathrm{NMR}\left(101 \mathrm{MHz}, \mathrm{CDCl}_{3}\right) \delta_{\mathrm{C}} 170.5\left(\mathrm{COCH}_{3}\right), 170.2\left(\mathrm{COCH}_{3}\right), 169.7\left(\mathrm{COCH}_{3}\right)$, 156.0 (NHCOOC), 134.0 (C8), 117.4 (C9), 83.9 (C1), 79.2, 77.5 (C3), 74.8 (C5), 70.5 (C6), 68.7 (C2), 66.4 (C4), 62.1 (C7), 39.0 (C12),

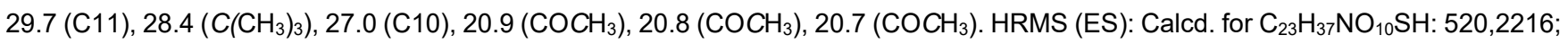
found. 520.2210 .

\section{Synthesis of oligosaccharide mimics from protected building blocks}

1-(tButyl(3-mercaptopropyl)carbamate) 2,4,6-tri-O-acetyl-3-O-[(2',4',6'-tri-O-acetyl-3'-O-[(2",3",4",6"-tetra-O-acetyl-1"thio- $\beta$-D-galactopyranosyl)propyl]- $\beta$-D-galactopyranosyl)-1H-[1,2,3]-triazol-4-yl-methyl]- $\beta$-D-galactopyranoside S34

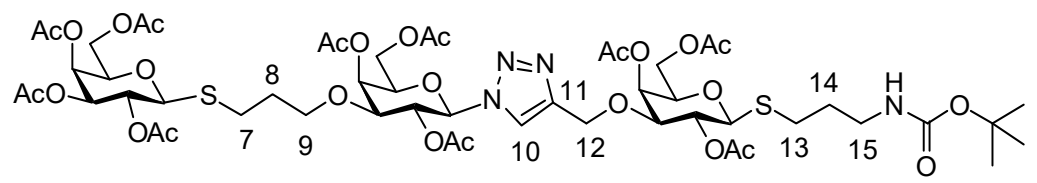

$\operatorname{DPAP}(3.1 \mathrm{mg} ; 12.6 \mu \mathrm{mol} ; 0.1$ equiv.) was added to a solution of thiol A2 (0.0506 g; $0.139 \mathrm{mmol} ; 1.1$ equiv.) and azide alkene B1 ( $0.0469 \mathrm{~g} ; 0.126 \mathrm{mmol} ; 1$ equiv.) dissolved in a $1: 1$ mixture of degassed $t \mathrm{BuOH} / \mathrm{H}_{2} \mathrm{O}(1.3 \mathrm{~mL})$ under a $\mathrm{N}_{2}$ atm. The mixture was irradiated at $360 \mathrm{~nm}$ overnight. When the reaction had run to completion the thioether alkyne D1 (0.0719 g; $0.139 \mathrm{mmol} ; 1.1 \mathrm{equiv}$.), $1 \mathrm{M}$ sodium ascorbate (aq) $\left(11.3 \mu \mathrm{L} ; 12.6 \mu \mathrm{mol} ; 0.1\right.$ equiv.) and $1 \mathrm{M} \mathrm{CuSO}_{4}(\mathrm{aq})(5.5 \mu \mathrm{L} ; 6.31 \mu \mathrm{mol} ; 0.05$ equiv.) were added and the mixture was heated to $70{ }^{\circ} \mathrm{C}$ overnight. When the reaction had run to completion the solvents were evaporated and the residue was purified by column chromatography (ethyl acetate/pentane 2:1). The product $\mathbf{S} 34$ was obtained as white foam in $57 \%$ yield $(0.090 \mathrm{~g} ; 0.0720 \mathrm{mmol})$. $R_{\mathrm{f}} 0.25$ (ethyl acetate/pentane 3:1). $[\alpha]_{\mathrm{D}}^{295 \mathrm{~K}}+7.2\left(c 1, \mathrm{CHCl}_{3}\right) .{ }^{1} \mathrm{H} \mathrm{NMR}\left(400 \mathrm{MHz}, \mathrm{CDCl}_{3}\right) \delta_{\mathrm{H}} 7.73(\mathrm{~s}, 1 \mathrm{H}, \mathrm{H} 10), 5.72(\mathrm{~d}, \mathrm{~J}=9.5 \mathrm{~Hz}, 1 \mathrm{H}$, 
H1'), 5.55 (s, 1H, H4/H4'), 5.45 (s, 1H, H4/H4'), 5.39 (d, J = 2.4 Hz, 1H, H4"), 5.33 (t, J = 9.5 Hz, 1H, H2'), 5.17 (t, J = 10.0 Hz, $1 \mathrm{H}$, H2"), 5.10 (t, J = 9.9 Hz, 1H, H2), $5.05-4.96(\mathrm{~m}, 1 \mathrm{H}, \mathrm{H} 3 "), 4.76(\mathrm{br} \mathrm{s}, 1 \mathrm{H}, \mathrm{NH}), 4.72(\mathrm{~d}, \mathrm{~J}=12.8 \mathrm{~Hz}, 1 \mathrm{H}, \mathrm{H} 12 \mathrm{a}), 4.62(\mathrm{~d}, \mathrm{~J}=12.8 \mathrm{~Hz}$, $1 \mathrm{H}, \mathrm{H} 12 \mathrm{~b}), 4.43(\mathrm{~d}, \mathrm{~J}=10.0 \mathrm{~Hz}, 1 \mathrm{H}, \mathrm{H} 1 "), 4.38(\mathrm{~d}, \mathrm{~J}=9.9 \mathrm{~Hz}, 1 \mathrm{H}, \mathrm{H} 1) .4 .22-3.99(\mathrm{~m}, 8 \mathrm{H}), 3.89(\mathrm{t}, \mathrm{J}=6.6 \mathrm{~Hz}, 1 \mathrm{H}), 3.82(\mathrm{t}, \mathrm{J}=6.4 \mathrm{~Hz}$, $1 \mathrm{H}$ ), $3.73(\mathrm{dd}, \mathrm{J}=8.2,5.6 \mathrm{~Hz}, 1 \mathrm{H}$ ), $3.66(\mathrm{~d}, \mathrm{~J}=9.5 \mathrm{~Hz}, 2 \mathrm{H}, \mathrm{H} 3$ and H3'), 3.48 (dd, $\mathrm{J}=13.9,6.6 \mathrm{~Hz}, 1 \mathrm{H}$ ), 3.17 (br s, $2 \mathrm{H}$ ), $2.80-2.51$ (m, $4 \mathrm{H}, \mathrm{H} 7$ and $\mathrm{H} 13), 2.17$ (s, 3H, $\left.\mathrm{COCH}_{3}\right), 2.13\left(\mathrm{~s}, 3 \mathrm{H}, \mathrm{COCH}_{3}\right), 2.12\left(\mathrm{~s}, 3 \mathrm{H}, \mathrm{COCH}_{3}\right), 2.06\left(\mathrm{~s}, 3 \mathrm{H}, \mathrm{COCH}_{3}\right), 2.03\left(\mathrm{~s}, 3 \mathrm{H}, \mathrm{COCH}_{3}\right)$, $2.03\left(\mathrm{~s}, 3 \mathrm{H}, \mathrm{COCH}_{3}\right), 2.02\left(\mathrm{~s}, 3 \mathrm{H}, \mathrm{COCH}_{3}\right), 2.01\left(\mathrm{~s}, 3 \mathrm{H}, \mathrm{COCH}_{3}\right) 1.95\left(\mathrm{~s}, 3 \mathrm{H}, \mathrm{COCH}_{3}\right), 1.89(\mathrm{~s}, 3 \mathrm{H}, \mathrm{COCH}), 1.83-1.68(\mathrm{~m}, 4 \mathrm{H}, \mathrm{H} 4$ and $\mathrm{H} 14), 1.40\left(\mathrm{~s}, 9 \mathrm{H}, \mathrm{OC}\left(\mathrm{CH}_{3}\right)_{3}\right) .{ }^{13} \mathrm{C} \mathrm{NMR}\left(101 \mathrm{MHz}, \mathrm{CDCl}_{3}\right) \delta_{\mathrm{C}} 170.6,170.5,170.4,170.2,170.1,170.0,169.8,169.6,169.1\left(\mathrm{COCH}_{3}\right)$, 156.0 (NHCOOC), 145.2 (C11), 121.2 (C10), 86.3 (C1'), 84.2 (C1/C1"), 83.8 (C1/C1"), 78.2 (C3/C3'), 77.8 (C3/C3'), 77.4, 74.8, 74.4, 74.3, 71.9 (C3"), 69.4 (C2'), 68.7 (C2), 68.6, 67.2 (C2"), 67.2 (C4/C4'/C4"), 66.4 (C4/C4'/C4"), 66.0 (C4/C4'/C4"), 63.1, 62.1, 61.8, 61.3, 39.1 (C9/C15), 29.8 (C8 and C14), $28.5\left(\mathrm{OC}\left(\mathrm{CH}_{3}\right)_{3}\right), 27.0$ (C7/C13), $26.6(\mathrm{C} 7 / \mathrm{C} 13), 21.0\left(\mathrm{COCH}_{3}\right), 20.9\left(\mathrm{COCH}_{3}\right), 20.8\left(\mathrm{COCH}_{3}\right), 20.8$ $\left(\mathrm{COCH}_{3}\right), 20.7\left(\mathrm{COCH}_{3}\right), 20.6\left(\mathrm{COCH}_{3}\right), 20.5\left(\mathrm{COCH}_{3}\right)$. HRMS (ES): Calcd. for $\mathrm{C}_{52} \mathrm{H}_{76} \mathrm{~N}_{4} \mathrm{O}_{27} \mathrm{~S}{ }_{2} \mathrm{H}$ : 1253.4217; found. 1253.4220.

1-(tButyl(3-mercaptopropyl)carbamate) 2,4,6-tri-O-acetyl-3-O-[(2',4',6'-tri-O-acetyl-3'-O-[(2",3",4",6"-tetra-O-acetyl- $\beta$-Dgalactopyranosyl)-1H-[1,2,3]-triazol-4-yl-methyl]-1'-thio- $\beta$-D-galactopyranosyl)propyl]- $\beta$-D-galactopyranoside $S 35$

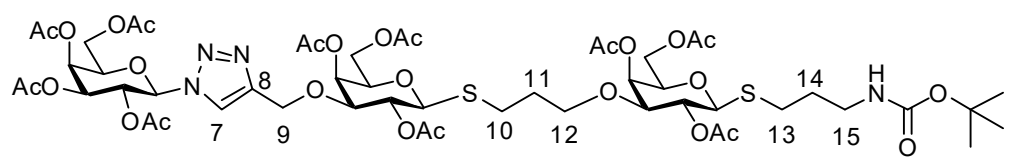

$1 \mathrm{M}$ Sodium ascorbate (aq) $\left(5.2 \mu \mathrm{L} ; 6.70 \mu \mathrm{mol} ; 0.1\right.$ equiv.) and $1 \mathrm{M} \mathrm{CuSO}_{4}$ (aq) $(2.6 \mu \mathrm{L} ; 3.35 \mu \mathrm{mol} ; 0.05$ equiv.) were added to a solution of azide A1 (0.0250 g; 0.067 mmol; 1 equiv.) and thioacetate $\mathbf{C} 1$ (0.0268 g; 0.0670 mmol; 1 equiv.) dissolved in a $1: 1$ mixture of degassed $t \mathrm{BuOH} / \mathrm{H}_{2} \mathrm{O}(0.8 \mathrm{~mL})$ under a $\mathrm{N}_{2}$ atm. The reaction mixture was heated to $70{ }^{\circ} \mathrm{C}$ overnight. When the reaction had reached completion it was allowed to cool to rt before DMAPA $(10.1 \mu \mathrm{L} ; 0.0804 \mathrm{mmol} ; 1.2$ equiv.) was added to remove the S-acetyl protecting group. The reaction was left at rt for one hour before it was diluted with dichloromethane and washed twice with $1 \mathrm{M} \mathrm{HCl}(\mathrm{aq})$ and brine, dried over magnesium sulfate, filtered and evaporated. The residue was re-dissolved in a 1:1 mixture of degassed $t \mathrm{BuOH} / \mathrm{H}_{2} \mathrm{O}(1 \mathrm{~mL})$ under a $\mathrm{N}_{2}$ atm. before the thioether alkene D2 (0.0358 g; $0.0688 \mathrm{mmol} ; 1$ equiv.) and DPAP (1.8 mg; $6.88 \mu \mathrm{mol} ; 0.1$ equiv.) were added. The mixture was irradiated at $360 \mathrm{~nm}$ overnight. When the reaction had reached completion the solvents were evaporated and the residue was purified by column chromatography (ethyl acetate/pentane 2:1). The product $\mathbf{S} 35$ was obtained as clear oil in $47 \%$ yield $\left(0.0406 \mathrm{~g} ; 0.0324 \mathrm{mmol}\right.$ ). $R_{\mathrm{f}} 0.42$ (ethyl acetate/pentane 4:1). $[\alpha]_{\mathrm{D}}{ }^{295 \mathrm{~K}}+6.6\left(c \mathrm{1}, \mathrm{CHCl}_{3}\right) .{ }^{1} \mathrm{H} \mathrm{NMR}\left(400 \mathrm{MHz}, \mathrm{CDCl}_{3}\right) \delta_{\mathrm{H}} 7.75(\mathrm{~s}$, $1 \mathrm{H}, \mathrm{H} 7$ ), 5.80 (d, J = 9.9 Hz, 1H, H1"), 5.54 (dd, $\mathrm{J}=9.9,8.0 \mathrm{~Hz}, 2 \mathrm{H}, \mathrm{H} 2 "$ and H4"), 5.46 (dd, J = 13.0, 2.8 Hz, 2H, H4 and H4'), 5.23 (dd, $J=9.9,3.2 \mathrm{~Hz}, 1 \mathrm{H}, \mathrm{H} 3 "), 5.12-5.03(\mathrm{~m}, 2 \mathrm{H}, \mathrm{H} 2$ and H2'), 4.75 (d, J = $12.6 \mathrm{~Hz}, 2 \mathrm{H}, \mathrm{NH}, \mathrm{H} 9 \mathrm{a}), 4.64$ (d, J = 12.6 Hz, 1H, H9b), 4.39 $\left(\mathrm{d}, \mathrm{J}=10.0 \mathrm{~Hz}, 1 \mathrm{H}, \mathrm{H} 1 / \mathrm{H} 1^{\prime}\right), 4.38\left(\mathrm{~d}, \mathrm{~J}=10.0 \mathrm{~Hz}, 1 \mathrm{H}, \mathrm{H} 1 / \mathrm{H} 1^{\prime}\right), 4.28-4.02(\mathrm{~m}, 7 \mathrm{H}), 3.83(\mathrm{t}, \mathrm{J}=6.4 \mathrm{~Hz}, 2 \mathrm{H}), 3.76-3.63\left(\mathrm{~m}, 2 \mathrm{H}, \mathrm{H} 3 / \mathrm{H} 3{ }^{\prime}\right)$, $3.48-3.35$ (m, 2H, H3/H3'), 3.20 (br s, 2H), $2.79-2.54\left(\mathrm{~m}, 4 \mathrm{H}, \mathrm{H} 10\right.$ and H13), $2.22\left(\mathrm{~s}, 3 \mathrm{H}, \mathrm{COCH}_{3}\right), 2.15\left(\mathrm{~s}, 3 \mathrm{H}, \mathrm{COCH}_{3}\right), 2.13(\mathrm{~s}$, $\left.3 \mathrm{H}, \mathrm{COCH}_{3}\right), 2.08\left(\mathrm{~s}, 3 \mathrm{H}, \mathrm{COCH}_{3}\right), 2.08\left(\mathrm{~s}, 3 \mathrm{H}, \mathrm{COCH}_{3}\right), 2.05$ (s, 3H, COCH $), 2.05\left(\mathrm{~s}, 3 \mathrm{H}, \mathrm{COCH}_{3}\right), 2.04(\mathrm{~s}, 3 \mathrm{H}, \mathrm{COCH}), 2.01(\mathrm{~s}, 3 \mathrm{H}$, $\left.\mathrm{COCH}_{3}\right), 1.88\left(\mathrm{~s}, 3 \mathrm{H}, \mathrm{COCH}_{3}\right), 1.85-1.69\left(\mathrm{~m}, 4 \mathrm{H}, \mathrm{H} 11\right.$ and H14), $1.42\left(\mathrm{~s}, 9 \mathrm{H}, \mathrm{OC}\left(\mathrm{CH}_{3}\right)_{3}\right) .{ }^{13} \mathrm{C} \mathrm{NMR}\left(101 \mathrm{MHz}, \mathrm{CDCl}_{3}\right) \delta_{\mathrm{C}} 170.6,170.6$, 170.5, 170.4, 170.3, 170.1, 169.9, 169.8, 169.7, 169.1 ( $\left.\mathrm{COCH}_{3}\right), 156.1$ (NHCOOC), 145.4 (C8), 121.4 (C7), 86.3 (C1"), 84.2 (C1/C1'), 84.0 (C1/C1'), 79.3 (C3/C3'), 78.2 (C3/C3'), 77.4, 74.9, 74.6, 74.1, 70.9 (C3"), 68.9 (C4/C4'), 68.8 (C4/C4'), $68.3,67.9$ (C2"/C3"), 67.0 (C2"/C3"), 66.4 (C4/C4'), 66.4 (C4/C4'), 63.3 (C9), 62.2, 62.0, 61.3, 39.2 (C12/C15), 29.9 (C11 and C14), $28.5\left(\mathrm{OC}\left(\mathrm{CH}_{3}\right)_{3}\right), 27.2$ (C10/C13), $26.8(\mathrm{C} 10 / \mathrm{C} 13), 21.1\left(\mathrm{COCH}_{3}\right), 21.0\left(\mathrm{COCH}_{3}\right), 20.9\left(\mathrm{COCH}_{3}\right), 20.9\left(\mathrm{COCH}_{3}\right), 20.9\left(\mathrm{COCH}_{3}\right), 20.8\left(\mathrm{COCH}_{3}\right), 20.6\left(\mathrm{COCH}_{3}\right)$, $20.4\left(\mathrm{COCH}_{3}\right)$. HRMS (ES): Calcd. for $\mathrm{C}_{52} \mathrm{H}_{76} \mathrm{~N}_{4} \mathrm{O}_{27} \mathrm{~S}_{2} \mathrm{H}$ : 1253.4217 found. 1253.4217.

1-(tButyl(3-mercaptopropyl)carbamate) 2,4,6-tri-O-acetyl-3-O-[(2'-acetamido-2'-deoxy-3',6'-O-acetyl-4'-O-[(2",3",4",6"-tetra-O-acetyl-1"-thio- $\beta$-D-galactopyranosyl)propyl]- $\beta$-D-glucopyranosyl)-1H-[1,2,3]-triazol-4-yl-methyl]- $\beta$-Dgalactopyranoside $\mathrm{S} 36$

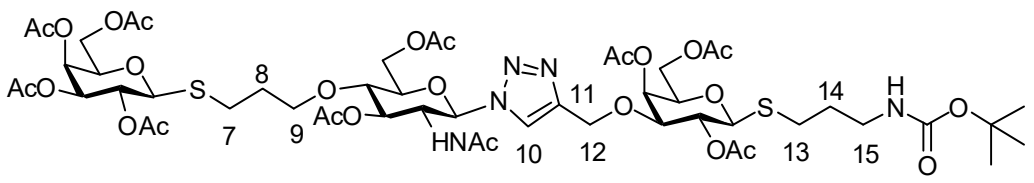


DPAP (3.3 mg; $13.0 \mu \mathrm{mol} ; 0.1$ equiv.) was added to a solution of thiol $\mathbf{A} 1$ ( $0.0475 \mathrm{~g} ; 0.130 \mathrm{mmol} ; 1$ equiv.) and GlcNAc azide-alkene B2 (0.0483 g; 0.130 mmol; 1 equiv.) dissolved in a $1: 1$ mixture of degassed $t \mathrm{BuOH} / \mathrm{H}_{2} \mathrm{O}(1.3 \mathrm{~mL})$ under a $\mathrm{N}_{2}$ atm. The mixture was irradiated at $360 \mathrm{~nm}$ overnight. When the reaction had reached completion the thioether alkyne D1 (0.0675 g; $0.130 \mathrm{mmol} ; 1 \mathrm{equiv}$ ), 1 M sodium ascorbate (aq) $\left(11.7 \mu \mathrm{L} ; 13.0 \mu \mathrm{mol} ; 0.1\right.$ equiv.) and $1 \mathrm{M} \mathrm{CuSO}_{4}$ (aq) $(5.7 \mu \mathrm{L} ; 6.52 \mu \mathrm{mol} ; 0.05$ equiv.) were added and the mixture was heated to $70^{\circ} \mathrm{C}$ overnight. When the reaction had reached completion, the solvents were evaporated and the residue was purified by column chromatography (ethyl acetate). The product $\mathbf{S} 36$ was obtained as a clear oil in $64 \%$ yield $(0.105 \mathrm{~g} ; 0.0834 \mathrm{mmol})$. $\mathrm{R}_{\mathrm{f}} 0.42$ (ethyl acetate/methanol 3:0.1). $[\alpha]_{\mathrm{D}}{ }^{295 \mathrm{~K}}-9.6\left(\mathrm{c} 1, \mathrm{CHCl}_{3}\right) .{ }^{1} \mathrm{H}$ NMR $\left(400 \mathrm{MHz}, \mathrm{CDCl}_{3}\right) \delta_{\mathrm{H}} 7.73(\mathrm{br} \mathrm{s}, 1 \mathrm{H}, \mathrm{H} 10), 6.23(\mathrm{br} \mathrm{d}, \mathrm{J}=7.7$ $\mathrm{Hz}, 1 \mathrm{H}, \mathrm{NH}), 5.86\left(\mathrm{~d}, \mathrm{~J}=9.8 \mathrm{~Hz}, 1 \mathrm{H}, \mathrm{H} 1^{\prime}\right), 5.47-5.34(\mathrm{~m}, 2 \mathrm{H}), 5.28(\mathrm{dd}, \mathrm{J}=13.0,6.5 \mathrm{~Hz}, 1 \mathrm{H}), 5.18(\mathrm{dd}, J=19.2,9.2 \mathrm{~Hz}, 1 \mathrm{H}), 5.08(\mathrm{t}$, $J=9.7 \mathrm{~Hz}, 1 \mathrm{H}), 5.02(\mathrm{dd}, \mathrm{J}=10.0,3.3 \mathrm{~Hz}, 1 \mathrm{H}), 4.84-4.55(\mathrm{~m}, 3 \mathrm{H}, \mathrm{NH}$ and H12), $4.51-4.30(\mathrm{~m}, 5 \mathrm{H}, \mathrm{H} 1, \mathrm{H} 1 "$ and H2'), $4.27-4.12$ $(\mathrm{m}, 2 \mathrm{H}), 4.09(\mathrm{~d}, \mathrm{~J}=6.3 \mathrm{~Hz}, 2 \mathrm{H}), 4.07-3.98(\mathrm{~m}, 1 \mathrm{H}), 3.93(\mathrm{t}, \mathrm{J}=6.6 \mathrm{~Hz}, 1 \mathrm{H}), 3.88-3.77(\mathrm{~m}, 2 \mathrm{H}), 3.77-3.64(\mathrm{~m}, 2 \mathrm{H}), 3.66-3.53$ $(\mathrm{m}, 2 \mathrm{H}), 3.17(\mathrm{~d}, \mathrm{~J}=5.1 \mathrm{~Hz}, 2 \mathrm{H}, \mathrm{H} 9 / \mathrm{H} 15), 2.82-2.55(\mathrm{~m}, 4 \mathrm{H}, \mathrm{H} 7$ and H13), 2.12, 2.12, 2.11, 2.06, 2.04, 2.03, 2.02, 2.01, 1.95 (9s, $\left.27 \mathrm{H}, 9 \mathrm{COCH}_{3}\right), 1.87-1.72(\mathrm{~m}, 4 \mathrm{H}, \mathrm{H} 8$ and $\mathrm{H} 14), 1.71\left(\mathrm{~s}, 3 \mathrm{H}, \mathrm{NHCOCH}_{3}\right), 1.39\left(\mathrm{~s}, 9 \mathrm{H}, \mathrm{OC}\left(\mathrm{CH}_{3}\right)_{3}\right) .{ }^{13} \mathrm{C} \mathrm{NMR}(101 \mathrm{MHz}, \mathrm{CDCl})_{3} \delta_{\mathrm{C}}$ $170.9\left(\mathrm{COCH}_{3}\right), 170.6\left(\mathrm{COCH}_{3}\right), 170.5\left(\mathrm{COCH}_{3}\right), 170.5\left(\mathrm{COCH}_{3}\right), 170.5\left(\mathrm{COCH}_{3}\right), 170.4\left(\mathrm{COCH}_{3}\right), 170.3\left(\mathrm{COCH}_{3}\right), 170.2\left(\mathrm{COCH}_{3}\right)$, $169.9\left(\mathrm{COCH}_{3}\right), 169.6\left(\mathrm{COCH}_{3}\right), 156.1(\mathrm{NHCOOC}), 144.8(\mathrm{C} 11), 121.9(\mathrm{C} 10), 86.2\left(\mathrm{C} 1{ }^{\prime}\right), 84.3(\mathrm{C} 1 / \mathrm{C} 1 "), 83.8(\mathrm{C} 1 / \mathrm{C} 1 "), 79.2,77.8$, 77.4, 76.2, 76.1, 74.8, 74.7, 74.4, 71.9, 71.1, 68.8, 67.3, 67.3, 66.6, 63.1 (C12), 62.5, 62.3, 61.4, 53.6, 39.1 (C9/C15), 30.4 (C8/C14), 29.8 (C8/C14), $28.5\left(\mathrm{OC}\left(\mathrm{CH}_{3}\right)_{3}\right), 27.0$ (C7/C13), 26.8 (C7/C13), $22.9\left(\mathrm{NHCOCH}_{3}\right), 21.1,21.0,20.9,20.8,20.8,20.7,20.6\left(\mathrm{COCH}_{3}\right)$. HRMS (ES): Calcd. for $\mathrm{C}_{52} \mathrm{H}_{77} \mathrm{~N}_{5} \mathrm{O}_{26} \mathrm{~S}_{2} \mathrm{H}$ : 1252.4376 ; found: 1252.4384 .

1-(tButyl(3-mercaptopropyl)carbamate) 2,4,6-tri-O-acetyl-3-O-[(2',4',6'-tri-O-acetyl-3'-O-[(2",3",4",6"-tetra-O-acetyl- $\alpha$-Dmannopyranosyl)-1H-[1,2,3]-triazol-4-yl-methyl]-1'-thio- $\beta$-D-galactopyranosyl)propyl]- $\beta$-D-galactopyranoside S37

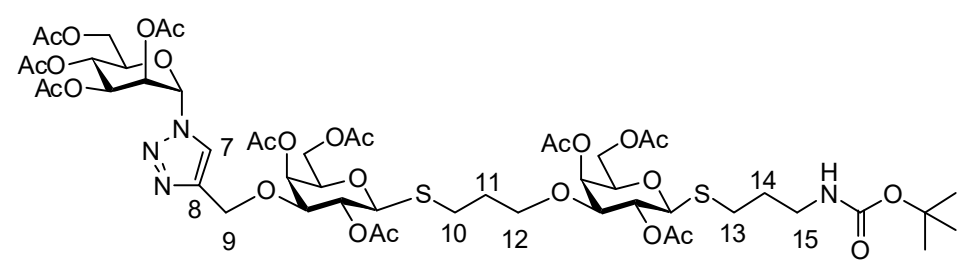

$1 \mathrm{M}$ Sodium ascorbate (aq) $\left(10.0 \mu \mathrm{L} ; 11.1 \mu \mathrm{mol} ; 0.1\right.$ equiv.) and $1 \mathrm{M} \mathrm{CuSO}_{4}$ (aq) $(4.9 \mu \mathrm{L} ; 5.56 \mu \mathrm{mol} ; 0.05$ equiv.) were added to a solution of azide $\mathbf{A} \mathbf{3}$ (0.0415 g; $0.111 \mathrm{mmol} ; 1$ equiv.) and thioacetate $\mathbf{C} 1$ (0.0447 g; $0.111 \mathrm{mmol} ; 1$ equiv.) dissolved in a $1: 1$ mixture of degassed $t \mathrm{BuOH} / \mathrm{H}_{2} \mathrm{O}(1.1 \mathrm{~mL})$ under a $\mathrm{N}_{2}$ atm. The reaction mixture was heated to $70^{\circ} \mathrm{C}$ overnight. When the reaction had reached completion it was allowed to cool to rt before DMAPA (16.8 $\mu \mathrm{L} ; 0.133 \mathrm{mmol} ; 1.2$ equiv.) was added to remove the S-acetyl protecting group. The reaction was left at rt for one hour before it was diluted with dichloromethane and washed twice with $1 \mathrm{M} \mathrm{HCl}(\mathrm{aq})$ and brine, dried over magnesium sulfate, filtered and evaporated. The residue was re-dissolved in a 1:1 mixture of degassed $t \mathrm{BuOH} / \mathrm{H}_{2} \mathrm{O}(1.1$ $\mathrm{mL}$ ) under a $\mathrm{N}_{2}$ atm. before the thioether alkene D2 (0.0578 g; $0.111 \mathrm{mmol} ; 1$ equiv.) and DPAP (2.8 mg; $11.1 \mu \mathrm{mol} ; 0.1$ equiv.) were added. The mixture was irradiated at $360 \mathrm{~nm}$ overnight. When the reaction had reached completion the solvents were evaporated and the residue was purified by column chromatography (ethyl acetate/pentane 2:1). The product $\mathbf{S} 3 \mathbf{7}$ was obtained as clear oil in $35 \%$ yield $\left(0.0494 \mathrm{~g} ; 0.0394 \mathrm{mmol}\right.$ ). $R_{\mathrm{f}} 0.43$ (ethyl acetate/pentane 4:1). $[\alpha]_{\mathrm{D}}^{295 \mathrm{~K}}+18.4\left(c 1, \mathrm{CHCl}_{3}\right) .{ }^{1} \mathrm{H} \mathrm{NMR}\left(400 \mathrm{MHz}, \mathrm{CDCl}_{3}\right) \delta_{\mathrm{H}} 7.67(\mathrm{~s}$, $1 \mathrm{H}, \mathrm{H} 7), 5.97$ (d, J = 2.6 Hz, 1H, H1"), $5.95-5.89(\mathrm{~m}, 1 \mathrm{H}), 5.86(\mathrm{dd}, \mathrm{J}=8.9,3.7 \mathrm{~Hz}, 1 \mathrm{H}), 5.44$ (dd, J = 6.6, 3.1 Hz, 2H), 5.35 (t, J = 8.9 $\mathrm{Hz}, 1 \mathrm{H}), 5.04(\mathrm{td}, \mathrm{J}=9.8,3.1 \mathrm{~Hz}, 2 \mathrm{H}), 4.74(\mathrm{~d}, \mathrm{~J}=12.7 \mathrm{~Hz}, 2 \mathrm{H}, \mathrm{NH}$ and $\mathrm{H} 9 \mathrm{a}), 4.66(\mathrm{~d}, \mathrm{~J}=12.7 \mathrm{~Hz}, 1 \mathrm{H}, \mathrm{H} 9 \mathrm{~b}), 4.41-4.29(\mathrm{~m}, 3 \mathrm{H}), 4.16$ - $3.99\left(\mathrm{~m}, 7 \mathrm{H}, \mathrm{H} 1\right.$ and $\left.\mathrm{H} 1^{\prime}\right), 3.95-3.86(\mathrm{~m}, 1 \mathrm{H}), 3.83(\mathrm{q}, \mathrm{J}=6.4 \mathrm{~Hz}, 2 \mathrm{H}), 3.75-3.63(\mathrm{~m}, 2 \mathrm{H}), 3.45-3.36(\mathrm{~m}, 2 \mathrm{H}), 3.19(\mathrm{~d}, \mathrm{~J}=4.7 \mathrm{~Hz}$, $2 \mathrm{H}, \mathrm{H} 12 / \mathrm{H} 15), 2.78-2.53\left(\mathrm{~m}, 4 \mathrm{H}, \mathrm{H} 10\right.$ and H13), $2.16\left(\mathrm{~s}, 3 \mathrm{H}, \mathrm{COCH}_{3}\right), 2.12\left(\mathrm{~s}, 3 \mathrm{H}, \mathrm{COCH}_{3}\right), 2.11\left(\mathrm{~s}, 3 \mathrm{H}, \mathrm{COCH}_{3}\right), 2.07\left(\mathrm{~s}, 3 \mathrm{H}, \mathrm{COCH}_{3}\right)$, $2.06\left(\mathrm{~s}, 3 \mathrm{H}, \mathrm{COCH}_{3}\right), 2.04\left(\mathrm{~s}, 3 \mathrm{H}, \mathrm{COCH}_{3}\right), 2.03\left(\mathrm{~s}, 3 \mathrm{H}, \mathrm{COCH}_{3}\right), 2.02\left(\mathrm{~s}, 3 \mathrm{H}, \mathrm{COCH}_{3}\right), 2.00\left(\mathrm{~s}, 3 \mathrm{H}, \mathrm{COCH}_{3}\right), 1.81-1.68(\mathrm{~m}, 4 \mathrm{H}, \mathrm{H} 11$ and $\mathrm{H} 14), 1.41$ (s, $\left.9 \mathrm{H}, \mathrm{OC}\left(\mathrm{CH}_{3}\right)_{3}\right) .{ }^{13} \mathrm{C} \mathrm{NMR}\left(101 \mathrm{MHz}, \mathrm{CDCl}_{3}\right) \delta_{\mathrm{C}} 170.6,170.6,170.4,170.3,169.8,169.7,169.4\left(\mathrm{COCH}_{3}\right), 156.0$ (NHCOOC), 145.5 (C8), 123.2 (C7), 84.2 (C1/C1'), 84.0 (C1/C1'), 83.6 (C1"), 79.3, 79.2, 78.5, 77.4, 74.8, 74.6, 72.2, 68.8, 68.8, 68.7, 68.3, 68.2, 66.5, 66.4, 66.0, 63.3 (C9), 62.2, 61.9, 61.5, 60.5, 39.1 (C12/C15), 29.8 (C11 and C14), $\left.28.5\left(\mathrm{OC}_{(\mathrm{CH}}\right)_{3}\right), 27.1(\mathrm{C} 10 / \mathrm{C} 13)$, $26.8(\mathrm{C} 10 / \mathrm{C} 13), 21.2\left(\mathrm{COCH}_{3}\right), 21.1\left(\mathrm{COCH}_{3}\right), 21.0\left(\mathrm{COCH}_{3}\right), 21.0\left(\mathrm{COCH}_{3}\right), 20.9\left(\mathrm{COCH}_{3}\right), 20.8\left(\mathrm{COCH}_{3}\right), 20.8\left(\mathrm{COCH}_{3}\right), 20.7$ $\left(\mathrm{COCH}_{3}\right)$. HRMS (ES): Calcd. for $\mathrm{C}_{52} \mathrm{H}_{76} \mathrm{~N}_{4} \mathrm{O}_{27} \mathrm{~S}_{2} \mathrm{H}$ : 1253.4217 ; found. 1253.4214. 
1-(tButyl(3-mercaptopropyl)carbamate) 2,4,6-tri-O-acetyl-3-O-[(2',4',6'-O-acetyl-3'-O-[(2"-acetamido-2"-deoxy-3",4",6"-O-acetyl -1"-thio- $\beta$-D-gluopyranosyl)propyl]- $\beta$-D-galactopyranosyl)-1H-[1,2,3]-triazol-4-yl-methyl]- $\beta$-Dgalactopyranoside S38

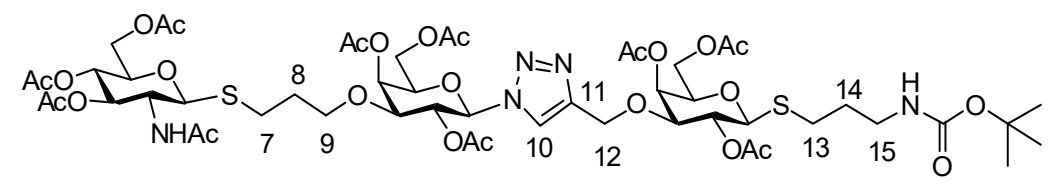

DPAP (2.1 mg; $8.28 \mu \mathrm{mol} ; 0.1$ equiv.) was added to a solution of thiol $\mathbf{A 6}(0.030 \mathrm{~g} ; 0.0828 \mathrm{mmol} ; 1$ equiv.) and azide-alkene B1 (0.0307 g; $0.0828 \mathrm{mmol} ; 1$ equiv.) dissolved in a $1: 1$ mixture of degassed $t \mathrm{BuOH} / \mathrm{H}_{2} \mathrm{O}(0.8 \mathrm{~mL})$ under a $\mathrm{N}_{2}$ atm. The mixture was irradiated at $360 \mathrm{~nm}$ overnight. When the reaction had run to completion the thioether alkyne D1 (0.0429 g; $0.0828 \mathrm{mmol} ; 1$ equiv.), $1 \mathrm{M}$ sodium ascorbate (aq) $\left(7.5 \mu \mathrm{L} ; 8.28 \mu \mathrm{mol} ; 0.1\right.$ equiv.) and $1 \mathrm{M} \mathrm{CuSO}_{4}$ (aq) $(3.6 \mu \mathrm{L} ; 4.14 \mu \mathrm{mol} ; 0.05$ equiv.) were added and the mixture was heated to $70{ }^{\circ} \mathrm{C}$ overnight. When the reaction had run to completion the solvents were evaporated and the residue was purified by column chromatography (ethyl acetate). The product $\mathbf{S} 38$ was obtained as a clear oil in $29 \%$ yield $(0.030 \mathrm{~g} ; 0.0240 \mathrm{mmol}) . R_{\mathrm{f}} 0.58(\mathrm{ethyl}$ acetate/methanol 3:0.3). $[\alpha]_{\mathrm{D}}{ }^{295 \mathrm{~K}}+3.6\left(\mathrm{c} 1, \mathrm{CHCl}_{3}\right) .{ }^{1} \mathrm{H}$ NMR $\left(400 \mathrm{MHz}, \mathrm{CDCl}_{3}\right) \delta_{\mathrm{H}} 7.74(\mathrm{~s}, 1 \mathrm{H}, \mathrm{H} 10), 5.90(\mathrm{~d}, \mathrm{~J}=9.5 \mathrm{~Hz}, 1 \mathrm{H}, \mathrm{NH}), 5.74$ $\left(\mathrm{d}, \mathrm{J}=9.5 \mathrm{~Hz}, 1 \mathrm{H}, \mathrm{H} 1^{\prime}\right), 5.57$ (d, J = 3.1 Hz, 1H, H4/H4'), 5.47 (d, J = 2.9 Hz, 1H, H4/H4'), 5.36 (t, J = 9.5 Hz, 1H, H2'), 5.19-5.02 (m, $3 \mathrm{H}), 4.77$ (s, 1H, NH), 4.74 (d, J = 12.8 Hz, 1H, H12a), 4.64 (d, J = 12.8 Hz, 1H, H12b), 4.55 (d, J = 10.4 Hz, 1H, H1/H1"), 4.40 (d, J = $10.0 \mathrm{~Hz}, 1 \mathrm{H}, \mathrm{H} 1 / \mathrm{H} 1 "), 4.27-4.00(\mathrm{~m}, 8 \mathrm{H}), 3.90-3.74(\mathrm{~m}, 2 \mathrm{H}), 3.74-3.60(\mathrm{~m}, 3 \mathrm{H}), 3.47(\mathrm{dd}, \mathrm{J}=15.1,6.3 \mathrm{~Hz}, 1 \mathrm{H}), 3.20(\mathrm{~d}, \mathrm{~J}=5.6$ $\mathrm{Hz}, 2 \mathrm{H}, \mathrm{H} 9 / \mathrm{H} 15), 2.82-2.52(\mathrm{~m}, 4 \mathrm{H}, \mathrm{H} 7$ and $\mathrm{H} 13), 2.22,2.15,2.08,2.06,2.05,2.04,2.01,2.01,1.94,1.91\left(10 \mathrm{~s}, 30 \mathrm{H}, \mathrm{NHCOCH}_{3}\right.$ and $\left.9 \mathrm{COCH}_{3}\right), 1.88-1.70\left(\mathrm{~m}, 4 \mathrm{H}, \mathrm{H} 8\right.$ and H14), $1.41\left(\mathrm{~s}, 9 \mathrm{H}, \mathrm{OC}\left(\mathrm{CH}_{3}\right)_{3}\right) .{ }^{13} \mathrm{C} \mathrm{NMR}\left(101 \mathrm{MHz}, \mathrm{CDCl}_{3}\right) \delta_{\mathrm{C}} 171.1,170.8,170.6,170.6,170.5$ 170.4, 170.2, 169.8, 169.4, $169.2\left(\mathrm{NHCOCH}_{3}\right.$ and $\left.\mathrm{COCH}_{3}\right), 156.1(\mathrm{NHCOOC}), 145.3(\mathrm{C} 11), 121.3(\mathrm{C} 10), 86.3$ (C1'), $84.3(\mathrm{C} 1 / \mathrm{C} 1 ")$, 83.9 (C1/C1"), 79.3, 78.3, 77.8, 77.4, 76.0, 74.8, 74.3, 73.9, 69.5 (C2'), 68.8, 68.7, 68.4, 66.4 (C4/C4"), 66.3 (C4/C4"), 63.2 (C12), 62.3, 62.2, 61.9, 53.2, 39.1, 29.8 (C8/C14), 29.1 (C8/C14), 28.5 (OC $\left.\left(\mathrm{CH}_{3}\right)_{3}\right), 27.1$ (C7/C13), 26.5 (C7/C13), 23.4, 21.0, 21.0, 20.9, 20.9, 20.8, 20.8, 20.7, $20.6\left(\mathrm{NHCOCH}_{3}\right.$ and $\left.\mathrm{COCH}_{3}\right)$. HRMS (ES): Calcd. for $\mathrm{C}_{52} \mathrm{H}_{77} \mathrm{~N}_{5} \mathrm{O}_{26} \mathrm{~S}_{2} \mathrm{H}$ : 1252.4376; found.1252.4387.

1-(tButyl(3-mercaptopropyl)carbamate) 2,4,6-tri-O-acetyl-3-O-[(2',4',6'-O-acetyl-3'-O-[(2",3",4",6"-tetra-O-acetyl-1"-thio$\alpha$-D-mannopyranosyl)propyl]- $\beta$-D-galactopyranosyl)-1H-[1,2,3]-triazol-4-yl-methyl]- $\beta$-D-galactopyranoside S39

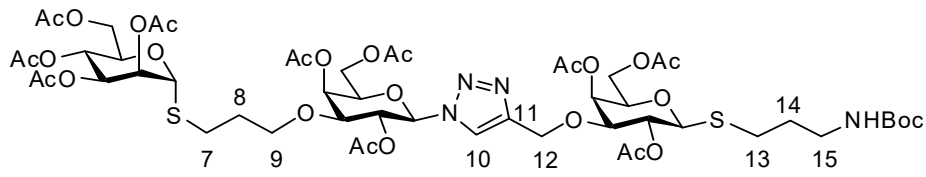

$\operatorname{DPAP}(2.0 \mathrm{mg} ; 7.08 \mu \mathrm{mol} ; 0.1$ equiv.) was added to a solution of thiol $\mathbf{A} 4$ ( $0.0258 \mathrm{~g} ; 0.0708 \mathrm{mmol} ; 1$ equiv.) and azide-alkene B1 (0.0263 g; 0.0708 mmol; 1 equiv.) dissolved in a $1: 1$ mixture of degassed $t \mathrm{BuOH} / \mathrm{H}_{2} \mathrm{O}(0.7 \mathrm{~mL})$ under a $\mathrm{N}_{2}$ atm. The mixture was irradiated at $360 \mathrm{~nm}$ overnight. When the reaction had reached completion the thioether alkyne D1 (0.0367 g; $0.0708 \mathrm{mmol} ; 1 \mathrm{equiv}$.), $1 \mathrm{M}$ sodium ascorbate (aq) $\left(6.4 \mu \mathrm{L} ; 7.08 \mu \mathrm{mol} ; 0.1\right.$ equiv.) and $1 \mathrm{M} \mathrm{CuSO}_{4}$ (aq) $(3.1 \mu \mathrm{L} ; 3.54 \mu \mathrm{mol} ; 0.05$ equiv.) were added and the mixture was heated to $70{ }^{\circ} \mathrm{C}$ overnight. When the reaction had reached completion the solvents were evaporated and the residue was purified by column chromatography (pentane/ethyl acetate 3:1). The product $\mathbf{S} 39$ was obtained as a clear oil in $33 \%$ yield (0.0296 $\mathrm{g}$; $0.0236 \mathrm{mmol}) R_{\mathrm{f}} 0.58$ (ethyl acetate). $[\alpha]_{\mathrm{D}}^{295 \mathrm{~K}}+26.4\left(\mathrm{c} 1, \mathrm{CHCl}_{3}\right) .{ }^{1} \mathrm{H} \mathrm{NMR}\left(400 \mathrm{MHz}, \mathrm{CDCl}_{3}\right) \delta_{\mathrm{H}} 7.74(\mathrm{~s}, 1 \mathrm{H}, \mathrm{H} 10), 5.76(\mathrm{~d}, \mathrm{~J}=9.4 \mathrm{~Hz}$, $\left.1 \mathrm{H}, \mathrm{H} 1^{\prime}\right), 5.59$ (d, J = $\left.2.5 \mathrm{~Hz}, 1 \mathrm{H}, \mathrm{H} 4^{\prime}\right), 5.47(\mathrm{~d}, \mathrm{~J}=2.2 \mathrm{~Hz}, 1 \mathrm{H}, \mathrm{H} 4), 5.41-5.26\left(\mathrm{~m}, 3 \mathrm{H}, \mathrm{H} 2^{\prime}\right), 5.22\left(\mathrm{~s}, 2 \mathrm{H}, \mathrm{H} 1{ }^{\prime \prime}\right), 5.12(\mathrm{t}, \mathrm{J}=9.9 \mathrm{~Hz}, 1 \mathrm{H}$, $\mathrm{H} 2), 4.74(\mathrm{~d}, \mathrm{~J}=12.9 \mathrm{~Hz}, 2 \mathrm{H}, \mathrm{NH}$ and H12a), 4.65 (d, J = 12.9 Hz, 1H, H12b), 4.40 (d, J = 9.9 Hz, 1H, H1), $4.37-4.24(\mathrm{~m}, 2 \mathrm{H}), 4.24$ $-3.99(\mathrm{~m}, 6 \mathrm{H}), 3.90-3.77(\mathrm{~m}, 2 \mathrm{H}), 3.73\left(\mathrm{dd}, \mathrm{J}=9.9,2.5 \mathrm{~Hz}, 1 \mathrm{H}, \mathrm{H}^{\prime}\right), 3.68(\mathrm{dd}, \mathrm{J}=9.9,2.2 \mathrm{~Hz}, 1 \mathrm{H}, \mathrm{H} 3), 3.52(\mathrm{dd}, \mathrm{J}=12.8,8.3 \mathrm{~Hz}$, $1 \mathrm{H}), 3.20(\mathrm{~d}, \mathrm{~J}=5.4 \mathrm{~Hz}, 2 \mathrm{H}, \mathrm{H} 9 / \mathrm{H} 15), 2.80-2.56(\mathrm{~m}, 4 \mathrm{H}, \mathrm{H} 7$ and $\mathrm{H} 13), 2.19\left(1 \mathrm{C}, \mathrm{COCH}_{3}\right), 2.18\left(1 \mathrm{C}, \mathrm{COCH}_{3}\right), 2.15\left(1 \mathrm{C}, \mathrm{COCH}_{3}\right)$, $2.09\left(1 \mathrm{C}, \mathrm{COCH}_{3}\right), 2.07\left(1 \mathrm{C}, \mathrm{COCH}_{3}\right), 2.06\left(1 \mathrm{C}, \mathrm{COCH}_{3}\right), 2.05\left(1 \mathrm{C}, \mathrm{COCH}_{3}\right), 2.04\left(1 \mathrm{C}, \mathrm{COCH}_{3}\right), 1.99\left(1 \mathrm{C}, \mathrm{COCH}_{3}\right), 1.91\left(1 \mathrm{C}, \mathrm{COCH}_{3}\right)$, $1.87-1.67(\mathrm{~m}, 4 \mathrm{H}, \mathrm{H} 8$ and $\mathrm{H} 14), 1.42\left(\mathrm{~s}, 9 \mathrm{H}, \mathrm{OC}\left(\mathrm{CH}_{3}\right)_{3}\right) .{ }^{13} \mathrm{C} \mathrm{NMR}\left(101 \mathrm{MHz}, \mathrm{CDCl}_{3}\right) \delta_{\mathrm{C}} 170.7\left(\mathrm{COCH}_{3}\right), 170.6\left(\mathrm{COCH}_{3}\right), 170.6$ $\left(\mathrm{COCH}_{3}\right), 170.5\left(\mathrm{COCH}_{3}\right), 170.3\left(\mathrm{COCH}_{3}\right), 170.0\left(\mathrm{COCH}_{3}\right), 170.0\left(\mathrm{COCH}_{3}\right), 169.8\left(\mathrm{COCH}_{3}\right), 169.8\left(\mathrm{COCH}_{3}\right), 169.2\left(\mathrm{COCH}_{3}\right), 156.1$ ( $\mathrm{NHCOOC)}, 145.3$ (C11), 121.2 (C10), 86.4 (C1'), 83.9 (C1), 83.4 (C1"), 79.3, 78.2 (C3/C3'), 77.8 (C3/C3'), 77.4, 74.8, 74.4, 71.4, 69.5, 69.4, 69.2, 68.7 (C2), 68.0, 66.4 (C4), 66.0 (C4'), 63.2 (C12), 62.4, 62.2, 61.8, 39.2 (C9/C15), 29.8 (C8/C14), 29.5 (C8/C14), 28.5 
$\left(\mathrm{OC}\left(\mathrm{CH}_{3}\right)_{3}\right), 28.3$ (C7/C13), 27.0 (C7/C13), 21.1, 21.1, 20.9, 20.9, 20.8, 20.8, $20.5\left(\mathrm{COCH}_{3}\right)$. HRMS (ES): Calcd. for $\mathrm{C}_{52} \mathrm{H}_{76} \mathrm{~N}_{4} \mathrm{O}_{27} \mathrm{~S}_{2} \mathrm{H}$ : 1253.4217; found. 1253.4232 .

1-(tButyl(3-mercaptopropyl)carbamate) 2,4,6-tri-O-acetyl-3-O-[(2'-acetamido-2'-deoxy-3',6'-O-acetyl-4'-O-[(2",3",4",6"-tetra-O-acetyl-1"-thio- $\alpha$-D-mannopyranosyl)propyl]- $\beta$-D-glucopyranosyl)-1 H-[1,2,3]-triazol-4-yl-methyl]- $\beta$-Dgalactopyranoside $\mathbf{S 4 0}$

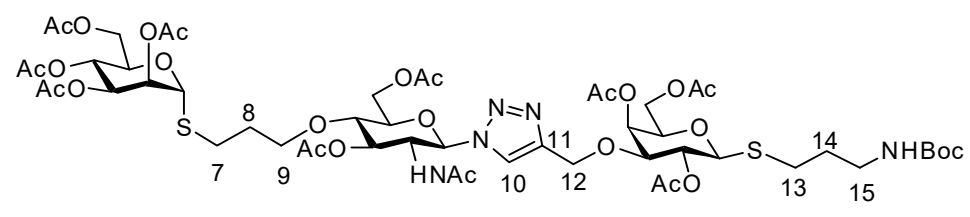

DPAP (1.7 mg; $6.53 \mu \mathrm{mol} ; 0.1$ equiv.) was added to a solution of thiol A4 (0.0238 g; 0.0653 mmol; 1 equiv.) and GlcNAc azide-alkene B1 ( $0.0243 \mathrm{~g} ; 0.0653 \mathrm{mmol} ; 1$ equiv.) dissolved in a $1: 1$ mixture of degassed $t \mathrm{BuOH} / \mathrm{H}_{2} \mathrm{O}(0.7 \mathrm{~mL})$ under a $\mathrm{N}_{2}$ atm. The mixture was irradiated at $360 \mathrm{~nm}$ overnight. When the reaction had reached completion the thioether alkyne D1 (0.0338 g; $0.0653 \mathrm{mmol} ; 1$ equiv.), $1 \mathrm{M}$ sodium ascorbate (aq) $\left(6.0 \mu \mathrm{L} ; 6.53 \mu \mathrm{mol} ; 0.1\right.$ equiv.) and $1 \mathrm{M} \mathrm{CuSO}_{4}$ (aq) $(2.9 \mu \mathrm{L} ; 3.27 \mu \mathrm{mol} ; 0.05$ equiv.) were added and the mixture was heated to $70^{\circ} \mathrm{C}$ overnight. When the reaction had reached completion the solvents were evaporated and the residue was purified by column chromatography (ethyl acetate). The product $\mathbf{S 4 0}$ was obtained as a clear oil in $45 \%$ yield $(0.0296 \mathrm{~g} ; 0.0236 \mathrm{mmol})$. $R_{\mathrm{f}} 0.31$ (ethyl acetate/methanol 3:0.1). [ $\left.\alpha\right]_{\mathrm{D}}{ }^{295 \mathrm{~K}}+15.6\left(\mathrm{c} 1, \mathrm{CHCl}_{3}\right) .{ }^{1} \mathrm{H} \mathrm{NMR}\left(400 \mathrm{MHz}, \mathrm{CDCl}_{3}\right) \delta_{\mathrm{H}} 7.73(\mathrm{~s}, 1 \mathrm{H}, \mathrm{H} 10), 6.02(\mathrm{~d}, \mathrm{~J}=9.4 \mathrm{~Hz}$, $1 \mathrm{H}, \mathrm{NH}), 5.84\left(\mathrm{t}, \mathrm{J}=9.1 \mathrm{~Hz}, 1 \mathrm{H}, \mathrm{H} 1^{\prime}\right), 5.44(\mathrm{~d}, \mathrm{~J}=2.8 \mathrm{~Hz}, 1 \mathrm{H}, \mathrm{H} 4), 5.36-5.25(\mathrm{~m}, 3 \mathrm{H}), 5.22(\mathrm{dd}, \mathrm{J}=9.7,3.6 \mathrm{~Hz}, 2 \mathrm{H}, \mathrm{H} 1 / \mathrm{H} 1 "), 5.10(\mathrm{t}$, $J=9.8 \mathrm{~Hz}, 1 \mathrm{H}), 4.77(\mathrm{br} \mathrm{s}, 1 \mathrm{H}, \mathrm{NH}), 4.72(\mathrm{~d}, \mathrm{~J}=12.8 \mathrm{~Hz}, 1 \mathrm{H}, \mathrm{H} 12 \mathrm{a}), 4.63(\mathrm{~d}, \mathrm{~J}=12.8 \mathrm{~Hz}, 1 \mathrm{H}, \mathrm{H} 12 \mathrm{~b}), 4.50-4.18(\mathrm{~m}, 6 \mathrm{H}, \mathrm{H} 1 / \mathrm{H} 1 "$ and $\left.\mathrm{H}^{\prime}\right), 4.11(\mathrm{~d}, \mathrm{~J}=6.7 \mathrm{~Hz}, 3 \mathrm{H}), 3.89-3.51(\mathrm{~m}, 6 \mathrm{H}, \mathrm{H} 3), 3.19(\mathrm{~d}, \mathrm{~J}=5.7 \mathrm{~Hz}, 2 \mathrm{H}), 2.80-2.51(\mathrm{~m}, 4 \mathrm{H}, \mathrm{H} 7$ and H13), $2.15(\mathrm{~s}, 3 \mathrm{H}), 2.14(\mathrm{~s}$, $\left.6 \mathrm{H}, 2 \mathrm{COCH}_{3}\right), 2.08\left(\mathrm{~s}, 3 \mathrm{H}, \mathrm{COCH}_{3}\right), 2.08\left(\mathrm{~s}, 3 \mathrm{H}, \mathrm{COCH}_{3}\right), 2.06\left(\mathrm{~s}, 3 \mathrm{H}, \mathrm{COCH}_{3}\right), 2.04\left(\mathrm{~s}, 6 \mathrm{H}, 2 \mathrm{COCH}_{3}\right), 1.98(\mathrm{~s}, 3 \mathrm{H}, \mathrm{COCH}), 1.89-$ $1.74(\mathrm{~m}, 4 \mathrm{H}, \mathrm{H} 8$ and $\mathrm{H} 14), 1.73\left(\mathrm{~s}, 3 \mathrm{H}, \mathrm{NHCOCH}_{3}\right), 1.41\left(\mathrm{~s}, 9 \mathrm{H}, \mathrm{OC}\left(\mathrm{CH}_{3}\right)_{3}\right) .{ }^{13} \mathrm{C} \mathrm{NMR}\left(101 \mathrm{MHz}, \mathrm{CDCl}_{3}\right) \delta_{\mathrm{C}} 171.0,170.7,170.6,170.6$, 170.5, 170.4, 170.2, 170.0, 169.9, $169.8\left(\mathrm{NHCOCH}_{3}\right.$ and $\left.\mathrm{COCH}_{3}\right), 156.1\left(\mathrm{NHCOOC}^{2}, 133.8(\mathrm{C} 11), 121.9(\mathrm{C} 10), 86.3(\mathrm{C} 1)^{\prime}\right), 83.9$ (C1/C1"), 82.1 (C1/C1"), 79.3, 77.7, 77.4, 76.3, 75.9, 74.9, 74.6, 71.1, 70.8, 69.5, 69.2, 68.8, 66.6 (C4), 66.3, 63.1 (C12), 62.5, 62.3, 53.7, 39.1, 29.8 (C8/C14), 29.5 (C8/C14), $28.5\left(\mathrm{OC}\left(\mathrm{CH}_{3}\right)_{3}\right), 27.5$ (C7/C13), 27.1 (C7/C13), $23.0\left(\mathrm{NHCOCH}_{3}\right), 21.1,21.1,21.0,20.9$, 20.9, 20.9, 20.8, $20.7\left(\mathrm{COCH}_{3}\right)$. HRMS (ES): Calcd. for $\mathrm{C}_{52} \mathrm{H}_{77} \mathrm{~N}_{5} \mathrm{O}_{26} \mathrm{~S}_{2} \mathrm{H}$ : 1252.4376; found.1252.4383.

1-(tButyl(3-mercaptopropyl)carbamate) 2,4,6-tri-O-acetyl-3-O-[(2'-acetamido-2'-deoxy-3',6'-O-acetyl-4'-O-[(2"'acetamido-2"-deoxy-3",4",6"-O-acetyl-1"-thio- $\beta$-D-glucopyranosyl)propyl]- $\beta$-D-glucopyranosyl)-1H-[1,2,3]-triazol-4-ylmethyl]- $\beta$-D-galactopyranoside S41

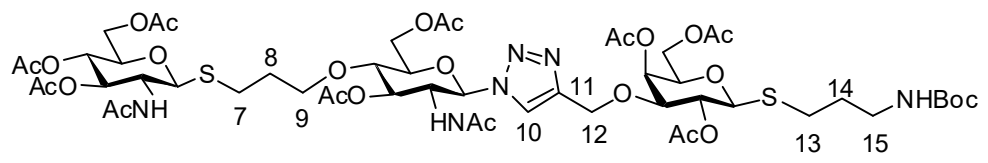

DPAP (1.9 mg; $7.34 \mu \mathrm{mol} ; 0.1$ equiv.) was added to a solution of thiol $\mathbf{A} 6$ (0.0267 g; $0.0734 \mathrm{mmol} ; 1$ equiv.) and GlcNAc azide-alkene B2 $\left(0.0272 \mathrm{~g} ; 0.0734 \mathrm{mmol} ; 1\right.$ equiv.) dissolved in a $1: 1$ mixture of degassed $t \mathrm{BuOH} / \mathrm{H}_{2} \mathrm{O}(0.8 \mathrm{~mL})$ under a $\mathrm{N}_{2}$ atm. The mixture was irradiated at $360 \mathrm{~nm}$ overnight. When the reaction had reached completion the thioether alkyne D1 (0.0380 g; $0.0734 \mathrm{mmol} ; 1$ equiv.), $1 \mathrm{M}$ sodium ascorbate (aq) $\left(6.9 \mu \mathrm{L} ; 7.34 \mu \mathrm{mol} ; 0.1\right.$ equiv.) and $1 \mathrm{M} \mathrm{CuSO}_{4}$ (aq) $(3.4 \mu \mathrm{L} ; 3.67 \mu \mathrm{mol} ; 0.05$ equiv.) were added and the mixture was heated to $70{ }^{\circ} \mathrm{C}$ overnight. When the reaction had reached completion the solvents were evaporated and the residue was purified by column chromatography (ethyl acetate/methanol 3:0.15). The product $\mathbf{S 4 1}$ was obtained as a white solid in $65 \%$ yield (0.0598 $\mathrm{g} ; 0.0478 \mathrm{mmol}) . R_{\mathrm{f}} 0.72$ (3:0.3 ethyl acetate/methanol). Decomposition point (uncorr) $208,1-215.0^{\circ} \mathrm{C}$. $[\alpha]_{\mathrm{D}}^{295 \mathrm{~K}}-26.8$ (c 0.5, methanol). ${ }^{1} \mathrm{H}$ NMR $\left(950 \mathrm{MHz}, d_{3}-\mathrm{MeOD}\right) \delta_{\mathrm{H}} 8.03(\mathrm{~s}, 1 \mathrm{H}, \mathrm{H} 10), 5.97(\mathrm{~d}, \mathrm{~J}=9.6 \mathrm{~Hz}, 1 \mathrm{H}, \mathrm{H} 1$ '), 5.53 (dd, J = 3.4, $0.9 \mathrm{~Hz}, 1 \mathrm{H}, \mathrm{H} 4), 5.34(\mathrm{t}, \mathrm{J}=9.6 \mathrm{~Hz}$, $\left.1 \mathrm{H}, \mathrm{H} 3^{\prime}\right), 5.19(\mathrm{t}, \mathrm{J}=9.7 \mathrm{~Hz}, 1 \mathrm{H}), 5.01-4.96(\mathrm{~m}, 2 \mathrm{H}), 4.70(\mathrm{~d}, \mathrm{~J}=4.4 \mathrm{~Hz}, 1 \mathrm{H}, \mathrm{H} 1 / \mathrm{H} 1 " / \mathrm{H} 12 \mathrm{a}), 4.69(\mathrm{~d}, J=1.7 \mathrm{~Hz}, 1 \mathrm{H}, \mathrm{H} 1 / \mathrm{H} 1 " / \mathrm{H} 12 \mathrm{a})$, $4.58(\mathrm{~d}, \mathrm{~J}=2.8 \mathrm{~Hz}, 1 \mathrm{H}, \mathrm{H} 1 / \mathrm{H} 1 " / \mathrm{H} 12 \mathrm{~b}), 4.57$ (s, 1H, H1/H1"/H12b), 4.47 (d, J = 10.4 Hz, 1H), 4.45 (dd, J = 12.3, $1.9 \mathrm{~Hz}, 2 \mathrm{H}), 4.28$ (dd, $J=12.4,5.2 \mathrm{~Hz}, 1 \mathrm{H}), 4.25(\mathrm{dd}, \mathrm{J}=12.3,5.1 \mathrm{~Hz}, 1 \mathrm{H}), 4.17-4.12(\mathrm{~m}, 2 \mathrm{H}), 4.10(\mathrm{dd}, \mathrm{J}=11.3,7.1 \mathrm{~Hz}, 1 \mathrm{H}), 4.01$ (ddd, J = 7.0, 5.9, 1.0 $\mathrm{Hz}, 1 \mathrm{H}$ ), $3.96(\mathrm{t}, \mathrm{J}=10.1 \mathrm{~Hz}, 1 \mathrm{H}$ ), 3.93 (ddd, $\mathrm{J}=9.9,5.2,2.0 \mathrm{~Hz}, 1 \mathrm{H}), 3.83(\mathrm{dd}, \mathrm{J}=9.6,3.4 \mathrm{~Hz}, 1 \mathrm{H}, \mathrm{H} 3$ ), $3.81-3.77(\mathrm{~m}, 2 \mathrm{H}), 3.71-$ 
$3.68(\mathrm{~m}, 1 \mathrm{H}), 3.67$ (dd, J = 15.5, $6.2 \mathrm{~Hz}, 1 \mathrm{H}), 3.16$ - 3.10 (m, 2H, H, H9/H15), 2.77 (dt, J = 14.2, 7.2 Hz, 1H, H7/H13), $2.75-2.68$ (m, $2 \mathrm{H}, \mathrm{H} 7 / \mathrm{H} 13), 2.66-2.61(\mathrm{~m}, 1 \mathrm{H}, \mathrm{H} 7 / \mathrm{H} 13), 2.14\left(\mathrm{~s}, 3 \mathrm{H}, \mathrm{COCH}_{3}\right), 2.11\left(\mathrm{~s}, 3 \mathrm{H}, \mathrm{COCH}_{3}\right), 2.09\left(\mathrm{~s}, 3 \mathrm{H}, \mathrm{COCH}_{3}\right), 2.07\left(\mathrm{~s}, 3 \mathrm{H}, \mathrm{COCH}_{3}\right), 2.05$ $\left(\mathrm{s}, 3 \mathrm{H}, \mathrm{COCH}_{3}\right), 2.01\left(\mathrm{~s}, 3 \mathrm{H}, \mathrm{COCH}_{3}\right), 1.98\left(\mathrm{~s}, 3 \mathrm{H}, \mathrm{COCH}_{3}\right), 1.98\left(\mathrm{~s}, 3 \mathrm{H}, \mathrm{COCH}_{3}\right), 1.90\left(\mathrm{~s}, 3 \mathrm{H}, \mathrm{NHCOCH}_{3}\right), 1.89-1.81(\mathrm{~m}, 3 \mathrm{H}, \mathrm{H} 8 / \mathrm{H} 14)$, $1.79-1.74(\mathrm{~m}, 2 \mathrm{H}, \mathrm{H} 8 / \mathrm{H} 14), 1.71\left(\mathrm{~s}, 3 \mathrm{H}, \mathrm{NHCOCH}_{3}\right), 1.43\left(\mathrm{~s}, 9 \mathrm{H}, \mathrm{OC}\left(\mathrm{CH}_{3}\right)_{3}\right) .{ }^{13} \mathrm{C} \mathrm{NMR}\left(239 \mathrm{MHz}, d_{3}-\mathrm{MeOD}\right) \delta_{\mathrm{C}} 173.2\left(\mathrm{NHCOCH}_{3}\right)$, $173.2\left(\mathrm{NHCOCH}_{3}\right), 172.4\left(\mathrm{COCH}_{3}\right), 172.3\left(\mathrm{COCH}_{3}\right), 172.2\left(\mathrm{COCH}_{3}\right), 172.1\left(\mathrm{COCH}_{3}\right), 171.9\left(\mathrm{COCH}_{3}\right), 171.9\left(\mathrm{COCH}_{3}\right), 171.6\left(\mathrm{COCH}_{3}\right)$, $171.3\left(\mathrm{COCH}_{3}\right), 158.5(\mathrm{NHCOOC}), 145.9(\mathrm{C} 11), 123.8(\mathrm{C} 10), 87.2\left(\mathrm{C}^{\prime}\right), 85.6,85.1,80.0,79.2,77.4,76.9,76.0,75.8,75.3,72.1,70.3$, 70.2, 68.4, 63.9, 63.6, 63.4 (C4), 54.9, 54.4, 40.2, 31.6 (C8/C14), 31.3 (C8/C14), $28.8\left(\mathrm{OC}\left(\mathrm{CH}_{3}\right)_{3}\right), 28.5$ (C7/C13), 28.0 (C7/C13), 22.7 $\left(\mathrm{NHCOCH}_{3}\right), 22.4\left(\mathrm{NHCOCH}_{3}\right), 21.1\left(\mathrm{COCH}_{3}\right), 21.0\left(\mathrm{COCH}_{3}\right), 20.8\left(\mathrm{COCH}_{3}\right), 20.7\left(\mathrm{COCH}_{3}\right), 20.7\left(\mathrm{COCH}_{3}\right), 20.7\left(\mathrm{COCH}_{3}\right), 20.6$ $\left(\mathrm{COCH}_{3}\right), 20.5\left(\mathrm{COCH}_{3}\right)$. HRMS (ES): Calcd. for $\mathrm{C}_{52} \mathrm{H}_{78} \mathrm{~N}_{6} \mathrm{O}_{25} \mathrm{~S}_{2} \mathrm{H}$ : 1251.4536; found.1251.4546.

1-(tButyl(3-mercaptopropyl)carbamate) 2,4,6-tri-O-acetyl-3-O-[(3',4',6'-O-acetyl-2'-O-[(2",3",4",6"--tetra-O-acetyl-1"thio$\alpha$-D-mannopyranosyl)propyl]- $\alpha$-D-mannopyranosyl)-1H-[1,2,3]-triazol-4-yl-methyl]- $\beta$-D-galactopyranoside $\$ 42$

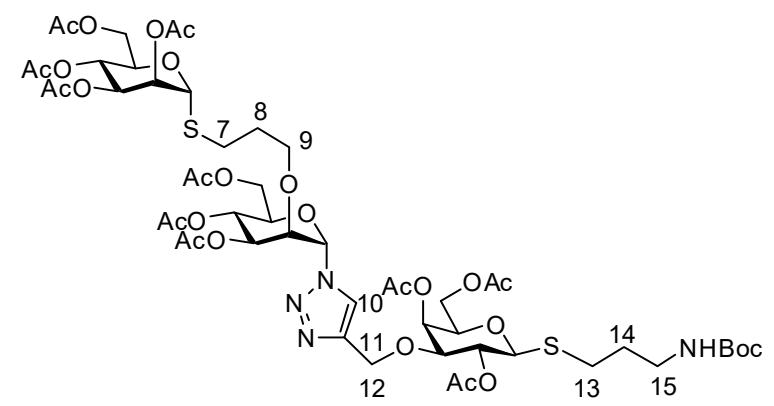

DPAP (1.2 mg; $4.77 \mu \mathrm{mol} ; 0.1$ equiv.) was added to a solution of thiol A4 (0.0174 g; $0.0477 \mathrm{mmol} ; 1$ equiv.) and mannosyl azide-alkene B3 ( $0.0177 \mathrm{~g}$; $0.0477 \mathrm{mmol} ; 1$ equiv.) dissolved in a $1: 1$ mixture of degassed $t \mathrm{BuOH} / \mathrm{H}_{2} \mathrm{O}(0.5 \mathrm{~mL})$ under a $\mathrm{N}_{2}$ atm. The mixture was irradiated at $360 \mathrm{~nm}$ overnight. When the reaction had reached completion the thioether alkyne D1 (0.0247 g; $0.0477 \mathrm{mmol} ; 1$ equiv.), $1 \mathrm{M}$ sodium ascorbate (aq) (4.3 $\square \mathrm{L} ; 4.77 \square \mathrm{mol} ; 0.1$ equiv.) and $1 \mathrm{M} \mathrm{CuSO}_{4}$ (aq) (2.1 $\mu \mathrm{L} ; 2.39 \mu \mathrm{mol} ; 0.05$ equiv.) were added and the mixture was heated to $70{ }^{\circ} \mathrm{C}$ overnight. When the reaction had reached completion the solvents were evaporated and the residue was purified by column chromatography (ethyl acetate/pentane 3:1). The product $\mathbf{S} 42$ was obtained as clear oil in $31 \%$ yield $(0.0187 \mathrm{~g}$; $0.0149 \mathrm{mmol}) . R_{\mathrm{f}} 0.65$ (ethyl acetate). $[\alpha]_{\mathrm{D}}{ }^{295 \mathrm{~K}}+46.2\left(\mathrm{c} 1, \mathrm{CHCl}_{3}\right) .{ }^{1} \mathrm{H} \mathrm{NMR}\left(400 \mathrm{MHz}, \mathrm{CDCl}_{3}\right) \delta_{\mathrm{H}} 7.73(\mathrm{~s}, 1 \mathrm{H}, \mathrm{H} 10), 5.95(\mathrm{~d}, \mathrm{~J}=3.8 \mathrm{~Hz}$, $\left.1 \mathrm{H}, \mathrm{H} 1^{\prime}\right), 5.61\left(\mathrm{dd}, \mathrm{J}=7.3,3.8 \mathrm{~Hz}, 1 \mathrm{H}, \mathrm{H} 3^{\prime}\right), 5.46(\mathrm{~d}, \mathrm{~J}=3.0 \mathrm{~Hz}, 1 \mathrm{H}, \mathrm{H} 4), 5.36-5.17\left(\mathrm{~m}, 4 \mathrm{H}, \mathrm{H} 1^{\prime}\right.$ "and H4'), $5.08(\mathrm{t}, \mathrm{J}=9.8 \mathrm{~Hz}, 1 \mathrm{H}, \mathrm{H} 2)$, $4.77\left(\mathrm{~d}, \mathrm{~J}=12.5 \mathrm{~Hz}, 2 \mathrm{H}, \mathrm{NH}\right.$ and H12a), $\left.4.67(\mathrm{~d}, \mathrm{~J}=12.5 \mathrm{~Hz}, 1 \mathrm{H}, \mathrm{H} 12 \mathrm{~b}), 4.60(\mathrm{t}, \mathrm{J}=3.8 \mathrm{~Hz}, 1 \mathrm{H}, \mathrm{H} 2)^{\prime}\right), 4.48-4.37(\mathrm{~m}, 2 \mathrm{H}, \mathrm{H} 1), 4.37-$ $4.24(\mathrm{~m}, 2 \mathrm{H}), 4.17-3.98(\mathrm{~m}, 4 \mathrm{H}), 3.96-3.82(\mathrm{~m}, 2 \mathrm{H}), 3.78-3.65(\mathrm{~m}, 2 \mathrm{H}, \mathrm{H} 3), 3.65-3.55(\mathrm{~m}, 1 \mathrm{H}), 3.20(\mathrm{~d}, \mathrm{~J}=4.5 \mathrm{~Hz}, 2 \mathrm{H}, \mathrm{H} 9 / \mathrm{H} 15)$, $2.80-2.54\left(\mathrm{~m}, 4 \mathrm{H}, \mathrm{H} 7\right.$ and H13), $2.15\left(\mathrm{~s}, 3 \mathrm{H}, \mathrm{COCH}_{3}\right), 2.14\left(\mathrm{~s}, 3 \mathrm{H}, \mathrm{COCH}_{3}\right), 2.12\left(\mathrm{~s}, 3 \mathrm{H}, \mathrm{COCH}_{3}\right), 2.08\left(\mathrm{~s}, 3 \mathrm{H}, \mathrm{COCH}_{3}\right), 2.07(\mathrm{~s}, 3 \mathrm{H}$, $\left.\mathrm{COCH}_{3}\right), 2.07\left(\mathrm{~s}, 3 \mathrm{H}, \mathrm{COCH}_{3}\right), 2.06\left(\mathrm{~s}, 3 \mathrm{H}, \mathrm{COCH}_{3}\right), 2.04\left(\mathrm{~s}, 3 \mathrm{H}, \mathrm{COCH}_{3}\right), 2.03\left(\mathrm{~s}, 3 \mathrm{H}, \mathrm{COCH}_{3}\right), 1.98\left(\mathrm{~s}, 3 \mathrm{H}, \mathrm{COCH}_{3}\right), 1.92-1.67(\mathrm{~m}$, $5 \mathrm{H}, \mathrm{H} 8$ and $\mathrm{H} 14), 1.42\left(\mathrm{~s}, 9 \mathrm{H}, \mathrm{OC}\left(\mathrm{CH}_{3}\right)_{3}\right) .{ }^{13} \mathrm{C} \mathrm{NMR}\left(101 \mathrm{MHz}, \mathrm{CDCl}_{3}\right) \delta_{\mathrm{C}} 170.8,170.7,170.6,170.4,170.2,169.9,169.9,169.8,169.6$ $\left(\mathrm{COCH}_{3}\right), 156.1$ (NHCOOC), 145.3 (C11), 123.5 (C10), 84.0 (C1), 83.6 (C1'), 82.9 (C1"), 79.3, 78.5 (C3), 77.4, 74.8, 74.7, 72.8, 72.5, 71.2, 69.6 (C3'), 69.5, 69.3 (C9/C15), 69.2, 68.8 (C2), 67.1, 66.8 (C4), 66.3, 63.5 (C12), 62.5, 62.2, 61.5, 39.1 (C9/C15), 29.8 (C8/C14), 29.3 (C8/C14), $28.5\left(\mathrm{OC}\left(\mathrm{CH}_{3}\right)_{3}\right), 27.9$ (C7/C13), 27.2 (C7/C13) 21.0, 21.0, 21.0, 21.0, 20.9, 20.9, 20.8, 20.8, 20.8 (COCH $) . \mathrm{HRMS}$ (ES): Calcd. for $\mathrm{C}_{52} \mathrm{H}_{76} \mathrm{~N}_{4} \mathrm{O}_{27} \mathrm{~S}_{2} \mathrm{H}$ : 1253.4217; found.1253.4220.

1-(tButyl(3-mercaptopropyl)carbamate) 2,4,6-tri-O-acetyl-3-O-[(2',4',6'-O-acetyl-3'-O-[(2",4",6"-tri-O-acetyl-3"-O[(2"',3"',4"',6"'-tetra-O-acetyl-1'"-thio- $\beta$-D-galactopyranosyl)propyl]- $\beta$-D-galactopyranosyl)-1H-[1,2,3]-triazol-4-ylmethyl]-1'-thio- $\beta$-D-galactopyranosyl)propyl]- $\beta$-D-galactopyranoside S43

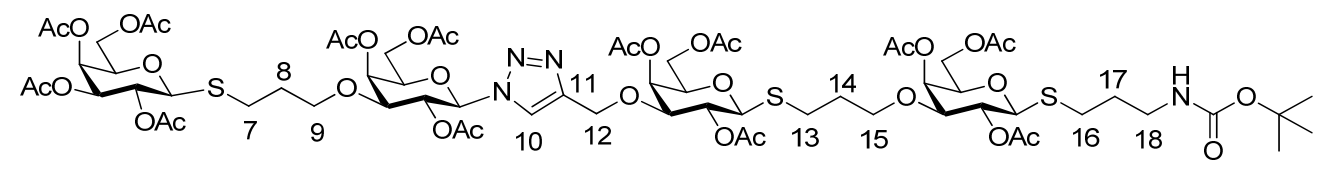

DPAP (3.1 mg; $11.9 \mu \mathrm{mol} ; 0.1$ equiv.) was added to a solution of thiol A2 $(0.0435 \mathrm{~g} ; 0.119 \mathrm{mmol} ; 1$ equiv.) and azide-alkene B1 (0.0443 g; 0.119 mmol; 1 equiv.) dissolved in a $1: 1$ mixture of degassed $t \mathrm{BuOH} / \mathrm{H}_{2} \mathrm{O}(1 \mathrm{~mL})$ under a $\mathrm{N}_{2}$ atm. The mixture was irradiated at 360 $\mathrm{nm}$ overnight. When the reaction had reached completion the thioacetate $\mathbf{C} 1$ ( $0.0480 \mathrm{~g} ; 0.119 \mathrm{mmol} ; 1$ equiv. $), 1 \mathrm{M}$ sodium ascorbate 
(aq) $\left(10.7 \mu \mathrm{L} ; 11.9 \mu \mathrm{mol} ; 0.1\right.$ equiv.) and $1 \mathrm{M} \mathrm{CuSO}_{4}$ (aq) $(5.2 \mu \mathrm{L} ; 5.95 \mu \mathrm{mol} ; 0.05$ equiv.) were added and the mixture was heated to $70^{\circ} \mathrm{C}$ overnight. When the reaction had reached completion it was allowed to cool to rt before DMAPA (18.0 $\mu \mathrm{L} ; 0.143 \mathrm{mmol} ; 1.2 \mathrm{equiv}$.) was added to remove the S-acetyl protecting group. After one hour the reaction was diluted with dichloromethane and washed twice with $1 \mathrm{M} \mathrm{HCl}(\mathrm{aq})$ and brine, dried over magnesium sulfate, filtered and evaporated. The residue was re-dissolved in a 1:1 mixture of degassed $t \mathrm{BuOH} / \mathrm{H}_{2} \mathrm{O}(1 \mathrm{~mL})$ under a $\mathrm{N}_{2}$ atm. before the thioether alkene D2 (0.0619 g; $0.119 \mathrm{mmol} ; 1$ equiv.) and DPAP (3.1 mg; 11.9 $\square$ mol; 0.1 equiv.) were added. The mixture was irradiated at $360 \mathrm{~nm}$ overnight. More DPAP (3.1 mg; $11.9 \square$ mol; 0.1 equiv.) was added and the reaction was irradiated overnight again. When the reaction had reached completion the solvents were evaporated and the residue was purified by column chromatography (ethyl acetate/pentane 1:1 to 3:1). The product $\mathbf{S} 4 \mathbf{3}$ was obtained as clear oil in $34 \%$ yield $(0.0651 \mathrm{~g} ; 0.0403 \mathrm{mmol}) . R_{\mathrm{f}} 0.66$ (ethyl acetate). $[\alpha]_{\mathrm{D}}{ }^{295 \mathrm{~K}}+13.6\left(c 1, \mathrm{CHCl}_{3}\right) .{ }^{1} \mathrm{H} \mathrm{NMR}\left(400 \mathrm{MHz}, \mathrm{CDCl}_{3}\right) \delta_{\mathrm{H}} 7.73(\mathrm{~s}, 1 \mathrm{H}, \mathrm{H} 10)$, $5.73(\mathrm{~d}, \mathrm{~J}=9.4 \mathrm{~Hz}, 1 \mathrm{H}, \mathrm{H} 1 "), 5.56(\mathrm{~d}, \mathrm{~J}=3.1 \mathrm{~Hz}, 1 \mathrm{H}), 5.49-5.26(\mathrm{~m}, 4 \mathrm{H}, \mathrm{H} 2 "), 5.19(\mathrm{t}, \mathrm{J}=10.0 \mathrm{~Hz}, 1 \mathrm{H}), 5.13-4.98(\mathrm{~m}, 3 \mathrm{H}), 4.73(\mathrm{~d}$, $J=12.8 \mathrm{~Hz}, 2 \mathrm{H}, \mathrm{H} 12 \mathrm{a}), 4.62$ (d, J = $12.8 \mathrm{~Hz}, 1 \mathrm{H}, \mathrm{H} 12 \mathrm{~b}), 4.44$ (d, J = 9.9 Hz, 1H, H1/H1'/H1'"), 4.38 (d, J = 10.0 Hz, 1H, H1/H1'/H1'"), $4.37\left(\mathrm{~d}, \mathrm{~J}=10.0 \mathrm{~Hz}, 1 \mathrm{H}, \mathrm{d}, \mathrm{J}=9.9 \mathrm{~Hz}, 1 \mathrm{H}, \mathrm{H} 1 / \mathrm{H}^{\prime} / \mathrm{H} 1^{\prime \prime}\right), 4.24-4.00(\mathrm{~m}, 8 \mathrm{H}), 3.90(\mathrm{t}, \mathrm{J}=6.7 \mathrm{~Hz}, 1 \mathrm{H}), 3.82(\mathrm{t}, \mathrm{J}=6.1 \mathrm{~Hz}, 2 \mathrm{H}), 3.78-$ $3.71(\mathrm{~m}, 1 \mathrm{H}), 3.71-3.62(\mathrm{~m}, 3 \mathrm{H}), 3.53-3.46(\mathrm{~m}, 1 \mathrm{H}), 3.46-3.35(\mathrm{~m}, 1 \mathrm{H}), 3.19(\mathrm{~d}, \mathrm{~J}=5.3 \mathrm{~Hz}, 3 \mathrm{H}, \mathrm{H} 9 / \mathrm{H} 15 / \mathrm{H} 18), 2.82-2.54(\mathrm{~m}, 6 \mathrm{H}$, $\mathrm{H} 7, \mathrm{H} 13$ and H16), $2.43\left(\mathrm{~s}, 1 \mathrm{H}, \mathrm{COCH}_{3}\right), 2.19\left(\mathrm{~s}, 1 \mathrm{H}, \mathrm{COCH}_{3}\right), 2.13\left(\mathrm{~s}, 1 \mathrm{H}, \mathrm{COCH}_{3}\right), 2.12\left(\mathrm{~s}, 1 \mathrm{H}, \mathrm{COCH}_{3}\right), 2.11\left(\mathrm{~s}, 1 \mathrm{H}, \mathrm{COCH}_{3}\right), 2.07$ $\left(\mathrm{s}, 1 \mathrm{H}, \mathrm{COCH}_{3}\right), 2.06\left(\mathrm{~s}, 1 \mathrm{H}, \mathrm{COCH}_{3}\right), 2.04\left(\mathrm{~s}, 1 \mathrm{H}, \mathrm{COCH}_{3}\right), 2.03\left(\mathrm{~s}, 1 \mathrm{H}, \mathrm{COCH}_{3}\right), 2.02\left(\mathrm{~s}, 1 \mathrm{H}, \mathrm{COCH}_{3}\right), 2.01\left(\mathrm{~s}, 1 \mathrm{H}, \mathrm{COCH}_{3}\right), 1.97(\mathrm{~s}$, $\left.1 \mathrm{H}, \mathrm{COCH}_{3}\right), 1.96\left(\mathrm{~s}, 1 \mathrm{H}, \mathrm{COCH}_{3}\right), 1.90\left(\mathrm{~s}, 1 \mathrm{H}, \mathrm{COCH}_{3}\right), 1.85-1.66\left(\mathrm{~m}, 6 \mathrm{H}, \mathrm{H} 8, \mathrm{H} 14\right.$ and H17), $1.41\left(\mathrm{~s}, 9 \mathrm{H}, \mathrm{OC}(\mathrm{CH})_{3}\right) .{ }^{13} \mathrm{C} \mathrm{NMR}(101$ $\left.\mathrm{MHz}, \mathrm{CDCl}_{3}\right) \delta_{\mathrm{C}} 170.6,170.5,170.4,170.3,170.3,170.1,170.0,169.8,169.7,169.6,169.1\left(\mathrm{COCH}_{3}\right), 156.0(\mathrm{NHCOOC}), 145.2(\mathrm{C} 11)$, 129.9 , 128.2, 121.2 (1C, C10), 86.3 (C1"), 84.2 (C1/C1'/C1'"), 84.2 (C1/C1'/C1'"), 84.0 (C1/C1'/C1'"'), 79.2, 78.3, 78.1, 77.4, 74.8, 74.6, 74.5, 71.9, 70.5, 69.4, 68.7, 68.6, 68.3, 67.9, 67.2, 67.2, 66.4, 66.0, 63.2 (C12), 62.2, 62.0, 61.8, 61.3, 39.1, 29.8 (C8, C14 and C17), $28.5\left(\mathrm{OC}\left(\mathrm{CH}_{3}\right)_{3}\right), 27.1$ (C7/C13/C16), 26.7 (C7/C13/C16), 26.7 (C7/C13/C16), 21.1, 21.0, 20.9, 20.9, 20.8, 20.8, 20.7, 20.5 (COCH $)$. HRMS (ES): Calcd. for $\mathrm{C}_{67} \mathrm{H}_{98} \mathrm{~N}_{4} \mathrm{O}_{35} \mathrm{~S}_{3} \mathrm{H}$ : 1615.5252; found: 1615.5285 .

1-(tButyl(3-mercaptopropyl)carbamate) 2,4,6-tri-O-acetyl-3-O-[(2',4',6'-O-acetyl-3'-O-[(2",4",6"-tri-O-acetyl-3"-O[(2"',3'",4"',6"'-tetra-O-acetyl- $\beta$-D-galactopyranosyl)-1H-[1,2,3]-triazol-4-yl-methyl]-1"-thio- $\beta$-Dgalactopyranosyl)propyl]- $\beta$-D-galactopyranosyl)-1H-[1,2,3]-triazol-4-yl-methyl]- $\beta$-D-galactopyranoside S44

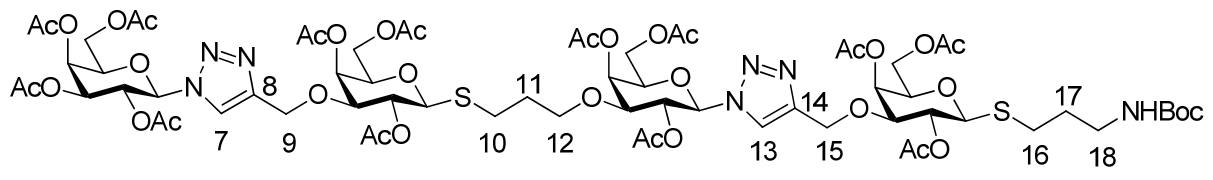

$1 \mathrm{M}$ Sodium ascorbate (aq) $\left(11.1 \mu \mathrm{L} ; 12.3 \mu \mathrm{mol} ; 0.1\right.$ equiv.) and $1 \mathrm{M} \mathrm{CuSO}_{4}$ (aq) $(5.4 \mu \mathrm{L} ; 6.15 \mu \mathrm{mol} ; 0.05$ equiv.) were added to a solution of azide $\mathbf{A} 1$ ( $0.0458 \mathrm{~g} ; 0.123 \mathrm{mmol} ; 1$ equiv.) and thioacetate $\mathbf{C} 1$ (0.0494 g; $0.123 \mathrm{mmol} ; 1$ equiv.) dissolved in a $1: 1 \mathrm{mixture}$ of degassed $t \mathrm{BuOH} / \mathrm{H}_{2} \mathrm{O}(1 \mathrm{~mL})$ under a $\mathrm{N}_{2}$ atm. The reaction mixture was heated to $70{ }^{\circ} \mathrm{C}$ overnight. When the reaction had reached completion it was allowed to cool to rt before DMAPA (18.6 $\mu \mathrm{L} ; 0.147 \mathrm{mmol} ; 1.2$ equiv.) was added to remove the S-acetyl protecting group. The reaction was left at rt for one hour before it was diluted with dichloromethane and washed twice with $1 \mathrm{M} \mathrm{HCl}(\mathrm{aq})$ and brine, dried over magnesium sulfate, filtered and evaporated.

In a separate flask $1 \mathrm{M}$ sodium ascorbate (aq) (11.1 $\mu \mathrm{L} ; 12.3 \mu \mathrm{mol} ; 0.1$ equiv.) and $1 \mathrm{M} \mathrm{CuSO}_{4}$ (aq) $(5.4 \mu \mathrm{L} ; 6.15 \mu \mathrm{mol} ; 0.05$ equiv.) were added to a solution of azide-alkene B1 (0.0456 g; $0.123 \mathrm{mmol} ; 1$ equiv.) and thioether alkyne D1 (0.0644 g; $0.123 \mathrm{mmol} ; 1 \mathrm{equiv}$.) dissolved in a 1:1 mixture of degassed $t \mathrm{BuOH} / \mathrm{H}_{2} \mathrm{O}(1 \mathrm{~mL})$ under a $\mathrm{N}_{2}$ atm. The reaction mixture was heated to $70^{\circ} \mathrm{C}$ overnight. When the reaction had reached completion the reaction the solvents were evaporated.

The two residues were mixed and re-dissolved in a 1:1 mixture of degassed $t \mathrm{BuOH} / \mathrm{H}_{2} \mathrm{O}(1 \mathrm{~mL})$ under a $\mathrm{N}_{2}$ atm. DPAP $(3.1 \mathrm{mg} ; 12.3$ $\mu \mathrm{mol} ; 0.1$ equiv.) was added and the reaction was irradiated at $360 \mathrm{~nm}$ overnight. More DPAP (3.1 mg; $12.3 \mu \mathrm{mol} ; 0.1$ equiv.) was added and the reaction was irradiated at $360 \mathrm{~nm}$ overnight again. When the reaction had reached completion the solvents were evaporated and the residue was purified by column chromatography (ethyl acetate/pentane to ethyl acetate 6:1). The product $\mathbf{S 4 4}$ was obtained as clear oil in $25 \%$ yield $\left(0.0505 \mathrm{~g} ; 0.0311 \mathrm{mmol}\right.$ ). $R_{\mathrm{f}} 0.70$ (ethyl acetate/methanol 3:0.1). $[\alpha]_{\mathrm{D}}{ }^{295 \mathrm{~K}}+19.2\left(c 1, \mathrm{CHCl}_{3}\right) .{ }^{1} \mathrm{H} \mathrm{NMR}$ (400 MHz, $\mathrm{CDCl}_{3}$ ) $\delta_{\mathrm{H}} 7.74$ (s, 2H, $\mathrm{H} 7$ and H13), 5.80 (d, J = 9.3 Hz, 1H, H1'/H1'"), 5.73 (dd, J = 9.3, $\left.4.3 \mathrm{~Hz}, 1 \mathrm{H}, \mathrm{H} 1^{\prime} / \mathrm{H} 1^{\prime \prime \prime}\right), 5.60-5.51$ $(\mathrm{m}, 3 \mathrm{H}), 5.47(\mathrm{~d}, \mathrm{~J}=3.1 \mathrm{~Hz}, 2 \mathrm{H}), 5.34(\mathrm{t}, \mathrm{J}=9.6 \mathrm{~Hz}, 1 \mathrm{H}), 5.23(\mathrm{dd}, \mathrm{J}=10.3,3.3 \mathrm{~Hz}, 1 \mathrm{H}), 5.10(\mathrm{q}, \mathrm{J}=9.5 \mathrm{~Hz}, 1 \mathrm{H}), 4.73(\mathrm{dd}, \mathrm{J}=12.8$, $2.9 \mathrm{~Hz}, 3 \mathrm{H}, \mathrm{NH}, \mathrm{H} 9 \mathrm{a}$ and $\mathrm{H} 15 \mathrm{a}), 4.68-4.56(\mathrm{~m}, 2 \mathrm{H}, \mathrm{H} 9 \mathrm{~b}$ and $\mathrm{H} 15 \mathrm{~b}), 4.43-4.33(\mathrm{~m}, 2 \mathrm{H}, \mathrm{H} 1 \mathrm{and} \mathrm{H} 1 "), 4.27-4.03(\mathrm{~m}, 12 \mathrm{H}), 3.82(\mathrm{dd}$, 
$\mathrm{J}=10.5,6.0 \mathrm{~Hz}, 2 \mathrm{H}), 3.78-3.62(\mathrm{~m}, 3 \mathrm{H}), 3.49(\mathrm{dd}, \mathrm{J}=15.2,6.5 \mathrm{~Hz}, 1 \mathrm{H}), 3.19(\mathrm{~d}, \mathrm{~J}=5.5 \mathrm{~Hz}, 2 \mathrm{H}, \mathrm{H} 12 / \mathrm{H} 18), 2.82-2.54(\mathrm{~m}, 4 \mathrm{H}, \mathrm{H} 10$ and $\mathrm{H} 16), 2.20\left(\mathrm{~s}, 3 \mathrm{H}, \mathrm{COCH}_{3}\right), 2.18\left(\mathrm{~s}, 3 \mathrm{H}, \mathrm{COCH}_{3}\right), 2.14\left(\mathrm{~s}, 3 \mathrm{H}, \mathrm{COCH}_{3}\right), 2.13\left(\mathrm{~s}, 3 \mathrm{H}, \mathrm{COCH}_{3}\right), 2.13\left(\mathrm{~s}, 3 \mathrm{H}, \mathrm{COCH}_{3}\right), 2.07(\mathrm{~s}, 3 \mathrm{H}$, $\left.\left.\mathrm{COCH}_{3}\right), 2.06\left(\mathrm{~s}, 3 \mathrm{H}, \mathrm{COCH}_{3}\right), 2.05\left(\mathrm{~s}, 3 \mathrm{H}, \mathrm{COCH}_{3}\right), 2.03\left(\mathrm{~s}, 3 \mathrm{H}, \mathrm{COCH}_{3}\right), 2.02\left(\mathrm{~s}, 3 \mathrm{H}, \mathrm{COCH}_{3}\right), 1.99 \mathrm{~s}, 3 \mathrm{H}, \mathrm{COCH}_{3}\right), 1.89(\mathrm{~s}, 3 \mathrm{H}$, $\left.\mathrm{COCH}_{3}\right), 1.87\left(\mathrm{~s}, 3 \mathrm{H}, \mathrm{COCH}_{3}\right), 1.76(\mathrm{dt}, \mathrm{J}=13.0,6.4 \mathrm{~Hz}, 4 \mathrm{H}, \mathrm{H} 11$ and $\mathrm{H} 17), 1.41\left(\mathrm{~s}, 9 \mathrm{H}, \mathrm{OC}\left(\mathrm{CH}_{3}\right)_{3}\right) .{ }^{13} \mathrm{C} \mathrm{NMR}\left(101 \mathrm{MHz}, \mathrm{CDCl}_{3}\right) \delta_{\mathrm{C}}$ 170.6, 170.5, 170.5, 170.4, 170.0, 170.0, 169.9, 169.8, 169.7, 169.1, $169.0\left(\mathrm{COCH}_{3}\right), 156.0\left(\mathrm{NHCOOC}^{2}, 145.3\right.$ (C8/C14), 145.2 (C8/C14), 121.4 (C7/C13), 121.2 (C7/C13), 86.3 (C1'/C1"'). 86.3 (C1'/C1'"), 84.0 (C1/C1"), 83.9 (C1/C1"), 78.2, 78.1, 77.8, 77.4, 75.0, 74.8, 74.7, 74.4, 74.1, 70.9, 69.5, 68.7, 67.8, 66.9, 66.4, 66.3, 66.0, 63.2 (C9/C15), 63.1 (C9/C15), 62.2, 61.9, 61.8, 61.2, 39.1 (C12/C18), 29.8 (C11 and C17), $28.5\left(\mathrm{OC}\left(\mathrm{CH}_{3}\right)_{3}\right), 27.1$ (C10/C16), 26.4 (C10/C16), 21.0, 20.9, 20.8, 20.8, 20.7, 20.6, 20.5, 20.4 $\left(\mathrm{COCH}_{3}\right)$. HRMS (ES): Calcd. for $\mathrm{C}_{67} \mathrm{H}_{95} \mathrm{~N}_{7} \mathrm{O}_{35} \mathrm{~S}_{2} \mathrm{H}$ : 1622.5389; found 1622.5425 .

1-(tButyl(3-mercaptopropyl)carbamate) 2,4,6-tri-O-acetyl-3-O-[(2'-acetamido-2'-deoxy-3',6'-O-acetyl-4'-O- [(2",4",6"-triO-acetyl-3"-O-[(2"',3"',4"',6"'-tetra-O-acetyl- $\alpha$-D-mannopyranosyl)-1H-[1,2,3]-triazol-4-yl-methyl]-1"-thio- $\beta$-Dgalactopyranosyl)propyl]- $\beta$-D-glucopyranosyl)-1H-[1,2,3]-triazol-4-yl-methyl]- $\beta$-D-galactopyranoside S45

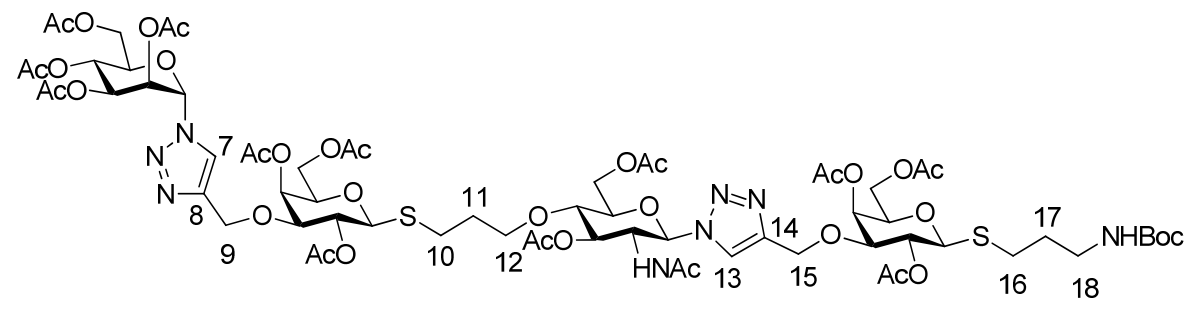

$1 \mathrm{M}$ Sodium ascorbate (aq) $\left(9.7 \mu \mathrm{L} ; 10.7 \mu \mathrm{mol} ; 0.1\right.$ equiv.) and $1 \mathrm{M} \mathrm{CuSO}_{4}$ (aq) (4.8 $\mu \mathrm{L} ; 5.35 \mu \mathrm{mol} ; 0.05$ equiv.) were added to a solution of azide A3 (0.0398 g; $0.107 \mathrm{mmol} ; 1$ equiv.) and thioacetate $\mathbf{C} 1$ ( $0.0429 \mathrm{~g} ; 0.107 \mathrm{mmol} ; 1$ equiv.) dissolved in a $1: 1 \mathrm{mixture}$ of degassed $t \mathrm{BuOH} / \mathrm{H}_{2} \mathrm{O}(1 \mathrm{~mL})$ under a $\mathrm{N}_{2}$ atm. The reaction mixture was heated to $70{ }^{\circ} \mathrm{C}$ overnight. When the reaction had reached completion it was allowed to cool to rt before DMAPA $(16.1 \mu \mathrm{L} ; 0.128 \mathrm{mmol} ; 1.2$ equiv.) was added to remove the S-acetyl protecting group. The reaction was left at rt for one hour before it was diluted with dichloromethane, washed once with brine, dried over magnesium sulfate, filtered and evaporated.

In a separate flask $1 \mathrm{M}$ sodium ascorbate (aq) $\left(9.7 \mu \mathrm{L} ; 10.7 \mu \mathrm{mol} ; 0.1\right.$ equiv.) and $1 \mathrm{M} \mathrm{CuSO}_{4}$ (aq) $(4.8 \mu \mathrm{L} ; 5.35 \mu \mathrm{mol} ; 0.05$ equiv.) were added to a solution of GlcNAc azide-alkene B2 (0.0395 g; $0.107 \mathrm{mmol} ; 1$ equiv.) and thioether alkyne D1 (0.0554 g; $0.107 \mathrm{mmol} ; 1$ equiv.) dissolved in a 1:1 mixture of degassed $t \mathrm{BuOH} / \mathrm{H}_{2} \mathrm{O}(1 \mathrm{~mL})$ under a $\mathrm{N}_{2}$ atm. The reaction mixture was heated to $70{ }^{\circ} \mathrm{C}$ overnight. When the reaction had reached completion the reaction the solvents were evaporated.

The two residues were mixed and re-dissolved in a 1:1 mixture of degassed $t \mathrm{BuOH} / \mathrm{H}_{2} \mathrm{O}(1 \mathrm{~mL})$ under a $\mathrm{N}_{2}$ atm. DPAP $(2.7 \mathrm{mg} ; 10.7$ $\mu \mathrm{mol} ; 0.1$ equiv.) was added and the reaction was irradiated at $360 \mathrm{~nm}$ overnight. More DPAP (2.7 mg; $10.7 \mu \mathrm{mol} ; 0.1$ equiv.) was added and the reaction was irradiated at $360 \mathrm{~nm}$ overnight again. When the reaction had reached completion the solvents were evaporated and the residue was purified by column chromatography (dichloromethane/methanol 3:0.075). The product $\mathbf{S 4 5}$ was obtained as clear oil in $22 \%$ yield $(0.0389 \mathrm{~g} ; 0.0240 \mathrm{mmol}) . R_{\mathrm{f}} 0.40$ (dichloromethane/methanol 3:0.1). $[\alpha]_{\mathrm{D}}{ }^{295 \mathrm{~K}}+9.0(\mathrm{c} 1, \mathrm{MeOH}) .{ }^{1} \mathrm{H}$ NMR (950 MHz, CDCl $) \delta_{\mathrm{H}} 7.74(\mathrm{~s}, 1 \mathrm{H}, \mathrm{H} 7 / \mathrm{H} 13), 7.68$ (s, 1H, H7/H13), 5.99 (d, J = 2.7 Hz, 1H, H1'/H1'"), 5.96 - 5.76 (m, 5H, H1'/H1'"), $5.47(\mathrm{~d}, \mathrm{~J}=2.9 \mathrm{~Hz}, 1 \mathrm{H}), 5.45(\mathrm{~d}, \mathrm{~J}=2.9 \mathrm{~Hz}, 1 \mathrm{H}), 5.37(\mathrm{t}, \mathrm{J}=8.9 \mathrm{~Hz}, 1 \mathrm{H}), 5.33-5.18(\mathrm{~m}, 2 \mathrm{H}), 5.08(\mathrm{dt}, \mathrm{J}=25.6,9.8 \mathrm{~Hz}, 2 \mathrm{H}), 4.81-$ $4.68(\mathrm{~m}, 3 \mathrm{H}), 4.65(\mathrm{t}, \mathrm{J}=9.8 \mathrm{~Hz}, 1 \mathrm{H}), 4.53-4.30(\mathrm{~m}, 5 \mathrm{H}, \mathrm{H} 1$ and $\mathrm{H} 1 "), 4.31-4.02(\mathrm{~m}, 7 \mathrm{H}), 3.96-3.81(\mathrm{~m}, 3 \mathrm{H}), 3.80-3.63(\mathrm{~m}, 3 \mathrm{H})$, $3.63-3.54(\mathrm{~m}, 1 \mathrm{H}), 3.21(\mathrm{~d}, \mathrm{~J}=5.6 \mathrm{~Hz}, 2 \mathrm{H}, \mathrm{H} 12 / \mathrm{H} 18), 2.82-2.60(\mathrm{~m}, 4 \mathrm{H}, \mathrm{H} 10$ and $\mathrm{H} 16), 2.18,2.15,2.14,2.13,2.12,2.09,2.09$, 2.07, 2.07, 2.07, 2.05, $2.02\left(12 \mathrm{~s}, 36 \mathrm{H}, \mathrm{COCH}_{3}\right), 1.87-1.71\left(\mathrm{~m}, 7 \mathrm{H}, \mathrm{H} 11, \mathrm{H} 17\right.$ and $\left.\mathrm{NHCOCH}_{3}\right), 1.42\left(\mathrm{~s}, 9 \mathrm{H}, \mathrm{OC}\left(\mathrm{CH}_{3}\right)_{3}\right) .{ }^{13} \mathrm{C} \mathrm{NMR}(239$ $\left.\mathrm{MHz}, \mathrm{CDCl}_{3}\right) \delta_{\mathrm{C}} 171.1,170.7,170.6,170.6,170.6,170.5,170.4,169.9,169.9,169.8,169.7,169.5\left(\mathrm{NHCOCH}_{3}\right.$ and $\left.\mathrm{COCH}_{3}\right), 156.1$ (NHCOOC), 145.7 (C8/C13), 145.0 (C8/C13), 133.8, 123.4 (C7/C14), 121.8 (C7/C14), 118.3, 86.5 (C1'/C1"'), 86.4 (C1'/C1'"'), 83.9 (C1/C1"), 83.7 (C1/C1"), 78.6, 77.8, 77.4, 76.4, 76.1, 75.3, 75.3, 74.9, 74.8, 74.7, 72.4, 71.2, 68.9, 68.9, 68.4, 66.7, 66.2, 63.4, 63.2, 62.6, 62.3, 62.0, 61.6, 53.9, 53.8, 39.2 (C12/18), 30.5 (C11/C17), 29.8 (C11/C17), $\left.28.6\left(\mathrm{OC}_{(} \mathrm{CH}_{3}\right)_{3}\right), 27.1$ (C10/C16), 26.8 (C10/C16), $23.0\left(\mathrm{NHCOCH}_{3}\right), 21.1,21.1,21.0,21.0,21.0,20.9,20.9,20.9,20.8,20.7\left(\mathrm{COCH}_{3}\right)$. HRMS (ES): Calcd. for $\mathrm{C}_{67} \mathrm{H}_{96} \mathrm{~N}_{8} \mathrm{O}_{34} \mathrm{~S}_{2} \mathrm{H}$ : 1621.5594; found.1621.5590.

\section{Deprotection of glycan mimics}




\section{General procedure A:}

The per-acetylated glycan was dissolved in anhydrous methanol $\left(1 \mathrm{~mL}\right.$ ) under a $\mathrm{N}_{2}$ atm. In a separate flask $0.1 \mathrm{~mL} 25 \% \mathrm{NaOMe}$ in methanol was added to anhydrous methanol $(2 \mathrm{~mL})$ under a $\mathrm{N}_{2}$ atm. $0.1 \mathrm{~mL}$ of the NaOMe solution was then added to the glycan. The reaction was left overnight at rt. When the reaction had reached completion methanol was evaporated.

1-(tButyl(3-mercaptopropyl)carbamate) 3-O-[(3'-O-[(1"-thio- $\beta$-D-galactopyranosyl)propyl]- $\beta$-D-galactopyranosyl)-1H$[1,2,3]$-triazol-4-yl-methyl]- $\beta$-D-galactopyranoside $\$ 46$

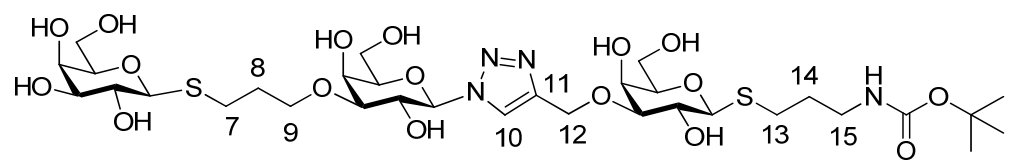

The product S46 was synthesized according to general procedure A and obtained as yellow oil $(0.0574 \mathrm{~g} ; 0.0689 \mathrm{mmol})$ in $96 \%$ yield $\mathrm{R}_{\mathrm{f}} 0.51$ (ethyl acetate/methanol 1:1). $[\alpha]_{\mathrm{D}}{ }^{295 \mathrm{~K}}+1.8\left(c\right.$ 1, MeOH). ${ }^{1} \mathrm{H}$ NMR $\left(400 \mathrm{MHz}, d_{3}-\mathrm{MeOD}\right) \delta_{\mathrm{H}} 8.22(\mathrm{~s}, 1 \mathrm{H}, \mathrm{H} 10), 5.57(\mathrm{~d}, \mathrm{~J}=9.2$ $\left.\mathrm{Hz}, 1 \mathrm{H}, \mathrm{H} 1^{\prime}\right), 4.83$ (d, J = 12.4 Hz, 1H, H12a), 4.76 (d, J = 12.4 Hz, 1H, H12b), 4.32 (s, 1H, H1/H1"), 4.30 (s, 1H, H1/H1"), $4.24-4.14$ $\left(\mathrm{m}, 2 \mathrm{H}, \mathrm{H} 2{ }^{\prime}\right), 4.08(\mathrm{~d}, \mathrm{~J}=2.7 \mathrm{~Hz}, 1 \mathrm{H}), 3.89-3.77(\mathrm{~m}, 3 \mathrm{H}), 3.77-3.60(\mathrm{~m}, 8 \mathrm{H}), 3.60-3.42(\mathrm{~m}, 5 \mathrm{H}), 3.38(\mathrm{dd}, \mathrm{J}=9.1,3.0 \mathrm{~Hz}, 1 \mathrm{H}), 3.27$ $(\mathrm{dt}, \mathrm{J}=3.1,1.6 \mathrm{~Hz}, 1 \mathrm{H}), 3.11(\mathrm{t}, \mathrm{J}=6.7 \mathrm{~Hz}, 2 \mathrm{H}, \mathrm{H} 9 / \mathrm{H} 15), 2.94-2.59(\mathrm{~m}, 4 \mathrm{H}, \mathrm{H} 7$ and H13), $1.98-1.88(\mathrm{~m}, 2 \mathrm{H}, \mathrm{H} 8 / \mathrm{H} 14), 1.81-1.69$ $(\mathrm{m}, 2 \mathrm{H}, \mathrm{H} 8 / \mathrm{H} 14), 1.39\left(\mathrm{~s}, 9 \mathrm{H}, \mathrm{OC}\left(\mathrm{CH}_{3}\right)_{3}\right) .{ }^{13} \mathrm{C} \mathrm{NMR}\left(101 \mathrm{MHz}, d_{3}-\mathrm{MeOD}\right) \delta_{\mathrm{C}} 158.5$ (NHCOOC), 146.4 (C11), 123.8 (C10), $90.2(\mathrm{C} 1 ')$, 87.8 (C1/C1"), 87.5 (C1/C1"), 83.9, 83.2, 80.6, 80.4, 79.9, 79.7, 76.2, 71.3, 70.6, 70.5, 69.1, 67.2, 67.0, 63.5 (C12), 62.8, 62.7, 62.4, 49.8, 40.2 (C9/C15), 31.2 (C8 and C14), $28.8\left(\mathrm{OC}\left(\mathrm{CH}_{3}\right)_{3}\right), 28.1(\mathrm{C} 7 / \mathrm{C} 13), 27.6$ (C7/C13). HRMS (ES): Calcd. for $\mathrm{C}_{32} \mathrm{H}_{56} \mathrm{~N}_{4} \mathrm{O}_{17} \mathrm{~S}_{2} \mathrm{Na}$ 855.2980; found. 855.2985 .

1-(tButyl(3-mercaptopropyl)carbamate) 3-O-[(3'-O-[( $\beta$-D-galactopyranosyl)-1H-[1,2,3]-triazol-4-yl-methyl]-1'-thio- $\beta$-Dgalactopyranosyl)propyl]- $\beta$-D-galactopyranoside S47

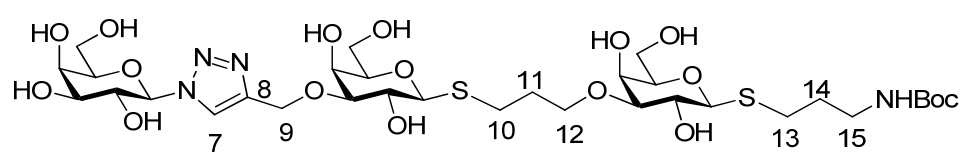

The product S47 was synthesized according to general procedure A and obtained as light yellow oil $(0.0378 \mathrm{~g} ; 0.0463 \mathrm{mmol})$ in quantitative yield. $R_{\mathrm{f}} 0.58$ (ethyl acetate/methanol 1:1). [ $\left.\alpha\right]_{\mathrm{D}}{ }^{295 \mathrm{~K}}-2.8\left(c 1, d_{3}-\mathrm{MeOD}\right) .{ }^{1} \mathrm{H} \mathrm{NMR}\left(400 \mathrm{MHz}, d_{3}-\mathrm{MeOD}\right) \delta_{\mathrm{H}} 8.22(\mathrm{~s}, 1 \mathrm{H}, \mathrm{H} 7)$, $5.54(\mathrm{~d}, \mathrm{~J}=7.7 \mathrm{~Hz}, 1 \mathrm{H}, \mathrm{H} 1$ ), $4.85-4.71$ (m, 2H, H9), 4.31 (dd, J = 14.6, 9.8 Hz, 2H), 4.07 (d, J = 10.2 Hz, 3H), 3.96 (s, 2H), 3.89 $2.99(\mathrm{~m}, 18 \mathrm{H}), 2.94-2.57\left(\mathrm{~m}, 4 \mathrm{H}, \mathrm{H} 10\right.$ and H13), $2.00-1.66(\mathrm{~m}, 4 \mathrm{H}, \mathrm{H} 11$ and $\mathrm{H} 14), 1.39\left(\mathrm{~s}, 9 \mathrm{H}, \mathrm{OC}\left(\mathrm{CH}_{3}\right)_{3}\right) .{ }^{13} \mathrm{C} \mathrm{NMR}\left(101 \mathrm{MHz}, d_{3}-\right.$ $\mathrm{MeOD}) \delta_{\mathrm{C}} 158.6(\mathrm{NHCOOC}), 146.4$ (C8), 123.9 (C7), 90.3 (C1"), 87.6 (C1 and C1'), 84.2, 84.0, 80.5, 79.9, 75.4, 71.5, 70.4, 70.4, 69.0, 67.3, 67.3, 63.5, 62.8, 62.7, 62.4, 49.9, 40.2, 31.2 (C11 and C14), $28.8\left(\mathrm{OC}\left(\mathrm{CH}_{3}\right)_{3}\right), 28.1(\mathrm{C} 11 / \mathrm{C} 14), 27.6(\mathrm{C} 11 / \mathrm{C} 14)$. HRMS (ES): Calcd. for $\mathrm{C}_{32} \mathrm{H}_{56} \mathrm{~N}_{4} \mathrm{O}_{17} \mathrm{~S}_{2} \mathrm{H}$ : 833.3160; found. 833.3161.

1-(tButyl(3-mercaptopropyl)carbamate 3-O-[(2'-acetamido-2'-deoxy-4'-O-[(1"-thio- $\beta$-D-galactopyranosyl)propyl]- $\beta$-Dglucopyranosyl)-1H-[1,2,3]-triazol-4-yl-methyl]- $\beta$-D-galactopyranoside S48

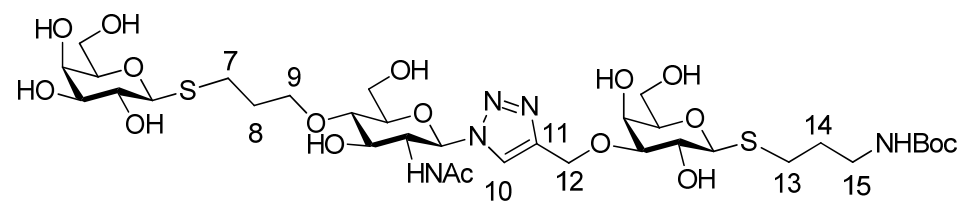

The product S48 was synthesized according to general procedure A and obtained as yellow oil $(0.0539 \mathrm{~g} ; 0.0617 \mathrm{mmol})$ in $93 \%$ yield. $R_{\mathrm{f}} 0.32$ (ethyl acetate/methanol 2:1.5). [ $\left.\alpha\right]_{\mathrm{D}}{ }^{295 \mathrm{~K}}-13.4(c 1, \mathrm{MeOH}) .{ }^{1} \mathrm{H}$ NMR $\left(400 \mathrm{MHz}, d_{3}-\mathrm{MeOD}\right) \delta_{\mathrm{H}} 8.24(\mathrm{~s}, 1 \mathrm{H}, \mathrm{H} 10), 5.75(\mathrm{~d}, \mathrm{~J}=9.8$ 
$\mathrm{Hz}, 1 \mathrm{H}, \mathrm{H} 1$ '), 4.84 (d, J = 12.6 Hz, 1H, H12a), 4.78 (d, J = $12.6 \mathrm{~Hz}, 1 \mathrm{H}, \mathrm{H} 12 \mathrm{~b}), 4.37$ (d, J = 9.5 Hz, 1H, H1/H1"), 4.34 (d, J = 9.5 Hz, $1 \mathrm{H}, \mathrm{H} 1 / \mathrm{H} 1 ") .4 .20\left(\mathrm{t}, \mathrm{J}=9.8 \mathrm{~Hz}, 1 \mathrm{H}, \mathrm{H} 2^{\prime}\right), 4.07(\mathrm{~s}, 1 \mathrm{H}), 4.04-3.96(\mathrm{~m}, 1 \mathrm{H}), 3.88(\mathrm{~d}, \mathrm{~J}=10.9 \mathrm{~Hz}, 2 \mathrm{H}), 3.86-3.64\left(\mathrm{~m}, 8 \mathrm{H}, \mathrm{H} 3^{\prime}\right), 3.64-$ $3.34(\mathrm{~m}, 8 \mathrm{H}), 3.31(\mathrm{~d}, \mathrm{~J}=1.4 \mathrm{~Hz}, 1 \mathrm{H}), 3.15(\mathrm{t}, \mathrm{J}=6.5 \mathrm{~Hz}, 2 \mathrm{H}, \mathrm{H} 9 / \mathrm{H} 15), 2.97-2.62(\mathrm{~m}, 4 \mathrm{H}, \mathrm{H} 7$ and H13), $2.04-1.86(\mathrm{~m}, 2 \mathrm{H}, \mathrm{H} 8 / \mathrm{H} 14)$, $1.79\left(\mathrm{~s}, 5 \mathrm{H}, \mathrm{H} 8 / \mathrm{H} 14\right.$ and $\left.\mathrm{NCOCH}_{3}\right), 1.43\left(\mathrm{~s}, 9 \mathrm{H}, \mathrm{OC}\left(\mathrm{CH}_{3}\right)_{3}\right) \cdot{ }^{13} \mathrm{C} \mathrm{NMR}\left(101 \mathrm{MHz}, d_{3}-\mathrm{MeOD}\right) \delta_{\mathrm{C}} 173.4\left(\mathrm{NHCOCH}_{3}\right), 158.5(\mathrm{NHCOOC})$, 146.3 (C11), 123.8 (C10), 88.2 (C1'), 87.6 (C1/C1"), 87.5 (C1/C1"), 83.6, 80.6, 80.4, 80.3, 79.9, 79.0, 76.2, 75.5, 72.3, 71.4, 70.6, 70.5, 67.4, 63.5 (C12), 62.7, 62.6, 61.9, 57.1, 40.2 (C9/C15), 31.6 (C8/C14), 31.2 (C8/C14), 28.8 (OC(CH $\left.)_{3}\right), 28.1$ (C7/C13), 27.6 (C7/C13), $22.7\left(\mathrm{NHCOCH}_{3}\right)$. HRMS (ES): Calcd. for $\mathrm{C}_{34} \mathrm{H}_{59} \mathrm{~N}_{5} \mathrm{O}_{17} \mathrm{~S}_{2} \mathrm{H}$ : 874.3426; found. 874.3425.

1-(tButyl(3-mercaptopropyl)carbamate) 3-O-[(-3'-O-[(- $\alpha-D-m a n n o p y r a n o s y l)-1 H-[1,2,3]-t r i a z o l-4-y l-m e t h y l]-1 '-t h i o-\beta-D-$ galactopyranosyl)propyl]- $\beta$-D-galactopyranoside S49

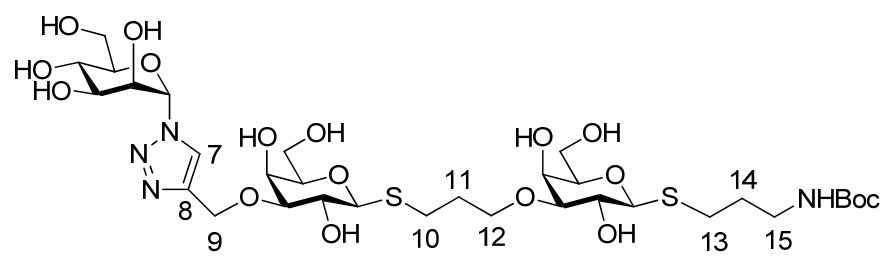

The product $\mathbf{S 4 9}$ was synthesized according to general procedure A and obtained as light yellow oil $(0.0314 \mathrm{~g} ; 0.0376 \mathrm{mmol})$ in $96 \%$ yield. $R_{\mathrm{f}} 0.29$ (ethyl acetate/methanol 2:1). $[\alpha]_{D}{ }^{295 K}+18.2(c 1, \mathrm{MeOH}) .{ }^{1} \mathrm{H}$ NMR $\left(400 \mathrm{MHz}, d_{3}-\mathrm{MeOD}\right) \delta_{\mathrm{H}} 8.17(\mathrm{~s}, 1 \mathrm{H}, \mathrm{H} 7), 6.00(\mathrm{~d}, J=$ $2.1 \mathrm{~Hz}, 1 \mathrm{H}, \mathrm{H} 1 "), 4.84$ (d, J = 12.4 Hz, 1H, H9a), 4.77 (d, J = $12.4 \mathrm{~Hz}, 1 \mathrm{H}, \mathrm{H} 9 \mathrm{~b}), 4.65\left(\mathrm{~s}, 1 \mathrm{H}, \mathrm{H} 2{ }^{\prime}\right), 4.33$ (d, J = 9.7 Hz, 1H, H1/H1'), $4.29\left(\mathrm{~d}, \mathrm{~J}=9.7 \mathrm{~Hz}, 1 \mathrm{H}, \mathrm{H} 1 / \mathrm{H} 1^{\prime}\right), 4.05(\mathrm{~s}, \mathrm{~J}=8.0 \mathrm{~Hz}, 2 \mathrm{H}), 4.01$ (dd, $\left.\mathrm{J}=8.4,3.1 \mathrm{~Hz}, 1 \mathrm{H}, \mathrm{H} 3^{\prime}\right), 3.87-3.54\left(\mathrm{~m}, 12 \mathrm{H}, \mathrm{H} 2, \mathrm{H} 2^{\prime}\right.$ and H5"), $3.48(\mathrm{dd}, \mathrm{J}=13.2,7.0 \mathrm{~Hz}, 2 \mathrm{H}), 3.38(\mathrm{dd}, \mathrm{J}=9.1,2.9 \mathrm{~Hz}, 1 \mathrm{H}), 3.29-3.24(\mathrm{~m}, 3 \mathrm{H}), 3.22(\mathrm{dd}, \mathrm{J}=9.2,3.1 \mathrm{~Hz}, 1 \mathrm{H}, \mathrm{H} 4 "), 3.11(\mathrm{t}, \mathrm{J}=6.7$ $\mathrm{Hz}, 2 \mathrm{H}, \mathrm{H} 12 / \mathrm{H} 15), 2.92-2.58(\mathrm{~m}, 4 \mathrm{H}, \mathrm{H} 10$ and $\mathrm{H} 13), 1.97-1.86(\mathrm{~m}, 2 \mathrm{H}, \mathrm{H} 11 / \mathrm{H} 14), 1.81-1.68(\mathrm{~m}, 2 \mathrm{H}, \mathrm{H} 11 / \mathrm{H} 14), 1.39(\mathrm{~s}, 9 \mathrm{H}$, $\left.\mathrm{OC}\left(\mathrm{CH}_{3}\right)_{3}\right) .{ }^{13} \mathrm{C}$ NMR (101 MHz, $\left.d_{3}-\mathrm{MeOD}\right) \delta_{\mathrm{C}} 158.6(\mathrm{NHCOOC}), 146.5(\mathrm{C} 8), 124.9(\mathrm{C} 7), 88.6(\mathrm{C} 1 "), 87.7$ (C1 and C1'), $84.3(\mathrm{C} 4 ")$, 84.1, 80.4, 79.9, 78.6, 72.7 (C3"), 70.4, 70.4, 70.2, 69.0, 68.8 (C2'), 67.4, 67.3, 63.6, 62.7, 62.6 (C12), 49.8, 40.2 (C12/C15), 31.2 (C11 and $\mathrm{C} 14)$, $28.8\left(\mathrm{OC}\left(\mathrm{CH}_{3}\right)_{3}\right), 28.1$ (C10/C13), 27.6 (C10/C13). HRMS (ES): Calcd. for $\mathrm{C}_{32} \mathrm{H}_{56} \mathrm{~N}_{4} \mathrm{O}_{17} \mathrm{~S}_{2} \mathrm{H}$ : 833.3160; found: 833.3161.

1-(tButyl(3-mercaptopropyl)carbamate 3-O-[(3'-O-[(2"-acetamido-2"-deoxy-1"-thio- $\beta$-D-gluopyranosyl)propyl]- $\beta$-Dgalactopyranosyl)-1H-[1,2,3]-triazol-4-yl-methyl]- $\beta$-D-galactopyranoside S50

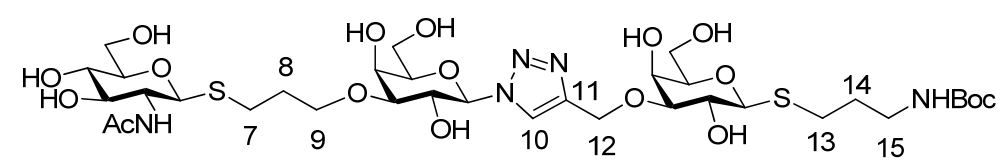

The product S50 was synthesized according to general procedure A and obtained as light brown foam $(0.0211 \mathrm{~g} ; 0.0241 \mathrm{mmol})$ in quantitative yield. $R_{\mathrm{f}} 0.26$ (ethyl acetate/methanol 2:1). [ $\left.\alpha\right]_{\mathrm{D}}{ }^{295 \mathrm{~K}}-10.2\left(c\right.$ 1, MeOH). ${ }^{1} \mathrm{H}$ NMR $\left(400 \mathrm{MHz}, d_{3}-\mathrm{MeOD}\right) \delta_{\mathrm{H}} 5.56(\mathrm{~d}, \mathrm{~J}=9.2$ $\left.\mathrm{Hz}, 1 \mathrm{H}, \mathrm{H} 1^{\prime}\right), 4.83$ (d, J = 12.4 Hz, 1H, H12a), 4.76 (d, J = 12.4 Hz, 1H, H12b), 4.46 (d, J = 10.3 Hz, 1H, H1/H1"), 4.30 (d, J = 9.7 Hz, $1 \mathrm{H}, \mathrm{H} 1 / \mathrm{H} 1 "), 4.22-4.13\left(\mathrm{~m}, 2 \mathrm{H}, \mathrm{H} 2{ }^{\prime}\right), 4.07(\mathrm{~d}, \mathrm{~J}=2.6 \mathrm{~Hz}, 1 \mathrm{H}), 3.88-3.57(\mathrm{~m}, 11 \mathrm{H}, \mathrm{H} 2$ and H2"), $3.52-3.33(\mathrm{~m}, 4 \mathrm{H}), 3.29-3.23(\mathrm{~m}$, $4 \mathrm{H}$ ), $3.11(\mathrm{t}, \mathrm{J}=6.6 \mathrm{~Hz}, 2 \mathrm{H}, \mathrm{H} 9 / \mathrm{H} 15), 2.94-2.57(\mathrm{~m}, 4 \mathrm{H}, \mathrm{H} 7$ and $\mathrm{H} 13), 1.97-1.83\left(\mathrm{~m}, 5 \mathrm{H}, \mathrm{NHCOCH}_{3}\right.$ and $\left.\mathrm{H} 8 / \mathrm{H} 14\right), 1.81-1.67(\mathrm{~m}$, $2 \mathrm{H}, \mathrm{H} 8 / \mathrm{H} 14), 1.39$ (s, 9H, OC( $\left.\left.\mathrm{CH}_{3}\right)_{3}\right) .{ }^{13} \mathrm{C}$ NMR (101 MHz, d $\left.d_{3}-\mathrm{MeOD}\right) \delta_{\mathrm{C}} 173.6\left(\mathrm{NHCOCH}_{3}\right), 158.7(\mathrm{NHCOOC}), 146.4(\mathrm{C} 11), 126.0$ (C10), 90.2 (C1'), 87.6 (C1/C1"), 85.8 (C1/C1"), 84.0, 83.3, 82.2, 80.5, 79.8, 77.4, 72.0, 70.5, 69.2, 67.3 (C12), 67.0, 63.6, 63.1, 62.7, 62.4, 56.2, 40.2 (C9/C15), 31.3 (C8/C14), 30.9 (C8/C14), $28.8\left(\mathrm{OC}\left(\mathrm{CH}_{3}\right)_{3}\right), 28.1(\mathrm{C} 7 / \mathrm{C} 13), 27.5(\mathrm{C} 7 / \mathrm{C} 13), 23.0(\mathrm{NHCOCH}) . \mathrm{HRMS}$ (ES): Calcd. for $\mathrm{C}_{34} \mathrm{H}_{59} \mathrm{~N}_{5} \mathrm{O}_{17} \mathrm{~S}_{2} \mathrm{H}: 874.3426$; found 874.3425

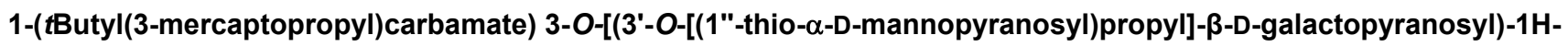
$[1,2,3]$-triazol-4-yl-methyl]- $\beta$-D-galactopyranoside S51 


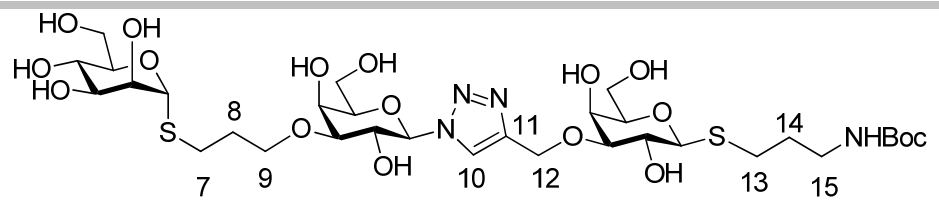

The product $\mathbf{S 5 1}$ was synthesized according to general procedure A and obtained as yellow oil $(0.0194 \mathrm{~g} ; 0.0233 \mathrm{mmol})$ in $99 \%$ yield. $R_{\mathrm{f}} 0.39$ (ethyl acetate/methanol 2:1.5). [ $\left.\alpha\right]_{\mathrm{D}}{ }^{295 \mathrm{~K}}+18.4$ (c 1, MeOH). ${ }^{1} \mathrm{H}$ NMR $\left(400 \mathrm{MHz}, d_{3}-\mathrm{MeOD} \delta_{\mathrm{H}} 8.25(\mathrm{~s}, 1 \mathrm{H}, \mathrm{H} 10), 5.60\right.$ (d, J = 9.1 $\mathrm{Hz}, 1 \mathrm{H}, \mathrm{H} 1$ '), 5.26 (s, 1H, H1"), 4.87 (d, J = 12.3 Hz, 1H, H12a), 4.80 (d, J = 12.3 Hz, 1H, H12b), 4.34 (d, J = 9.6 Hz, 1H, H1), $4.21(\mathrm{~d}$, $\left.\mathrm{J}=9.8 \mathrm{~Hz}, 2 \mathrm{H}, \mathrm{H} 2{ }^{\prime}\right), 4.11(\mathrm{~s}, 1 \mathrm{H}), 3.97-3.65(\mathrm{~m}, 13 \mathrm{H}, \mathrm{H} 2$ and $\mathrm{H} 2 "), 3.60-3.32(\mathrm{~m}, 5 \mathrm{H}), 3.16(\mathrm{~s}, 2 \mathrm{H}, \mathrm{H} 9 / \mathrm{H} 15), 2.92-2.61(\mathrm{~m}, 4 \mathrm{H}, \mathrm{H} 7$ and $\mathrm{H} 13), 2.11-1.71(\mathrm{~m}, 4 \mathrm{H}, \mathrm{H} 8$ and $\mathrm{H} 14), 1.44\left(\mathrm{~s}, 9 \mathrm{H}, \mathrm{OC}\left(\mathrm{CH}_{3}\right)_{3}\right) .{ }^{13} \mathrm{C}$ NMR (101 MHz, $\left.d_{3}-\mathrm{MeOD}\right) \delta_{\mathrm{C}} 158.6$ (NHCOOC), $146.5(\mathrm{C} 11)$, 123.7 (C10), 90.2 (C1'), 87.6 (C1), 86.6 (C1"), 84.0, 83.4, 80.4, 79.9, 79.8, 75.0, 73.8, 73.2, 70.5, 69.4, 68.9, 67.3, 67.1, 63.6 (C12), 62.8, 62.7, 62.4, 40.2 (C9/C15), 31.2 (C8/C14), 31.1 (C8/C14), $28.8\left(\mathrm{OC}\left(\mathrm{CH}_{3}\right)_{3}\right), 28.1$ (C7 and C13). HRMS (ES): Calcd. for $\mathrm{C}_{32} \mathrm{H}_{56} \mathrm{~N}_{4} \mathrm{O}_{17} \mathrm{~S}_{2} \mathrm{H}$ : 833.3160; found. 833.3160.

1-(tButyl(3-mercaptopropyl)carbamate) 3-O-[(2'-acetamido-2'-deoxy-4'-O-[(1'-thio- $\alpha$-D-mannopyranosyl)propyl]- $\beta$-Dglucopyranosyl)-1H-[1,2,3]-triazol-4-yl-methyl]- $\beta$-D-galactopyranoside S52

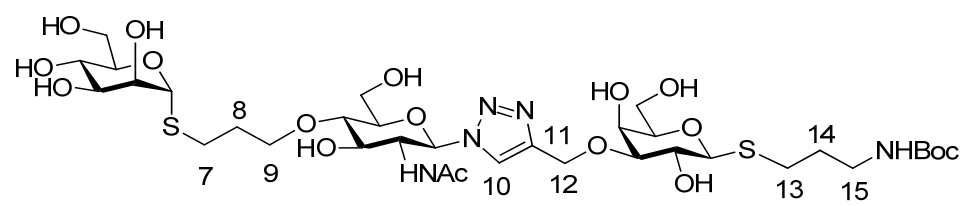

The product S52 was synthesized according to general procedure A and obtained as yellow oil $(0.0229 \mathrm{~g} ; 0.0262 \mathrm{mmol})$ in quantitative yield. $R_{\mathrm{f}} 0.36$ (ethyl acetate/methanol 2:1.5). $[\alpha]_{\mathrm{D}}{ }^{295 \mathrm{~K}}-76.2(c 1, \mathrm{MeOH}) .{ }^{1} \mathrm{H} \mathrm{NMR}\left(400 \mathrm{MHz}, d_{3}-\mathrm{MeOD}\right) \delta_{\mathrm{H}} 8.22(\mathrm{~s}, 1 \mathrm{H}, \mathrm{H} 10), 5.74(\mathrm{~d}, \mathrm{~J}$ $\left.=9.7 \mathrm{~Hz}, 1 \mathrm{H}, \mathrm{H} 1^{\prime}\right), 5.25(\mathrm{~s}, 1 \mathrm{H}, \mathrm{H} 1 "), 4.84(\mathrm{~d}, \mathrm{~J}=12.6 \mathrm{~Hz}, 1 \mathrm{H}, \mathrm{H} 12 \mathrm{a}), 4.78(\mathrm{~d}, \mathrm{~J}=12.6 \mathrm{~Hz}, 1 \mathrm{H}, \mathrm{H} 12 \mathrm{~b}), 4.36(\mathrm{~d}, \mathrm{~J}=9.7 \mathrm{~Hz}, 1 \mathrm{H}, \mathrm{H} 1)$, $4.19\left(\mathrm{t}, \mathrm{J}=10.0 \mathrm{~Hz}, 1 \mathrm{H}, \mathrm{H} 2{ }^{\prime}\right), 4.06-4.01(\mathrm{~m}, 2 \mathrm{H}), 3.96-3.55(\mathrm{~m}, 9 \mathrm{H}, \mathrm{H} 2, \mathrm{H} 2$ " and H3'), $3.57-3.50(\mathrm{~m}, 1 \mathrm{H}), 3.49-3.35(\mathrm{~m}, 2 \mathrm{H}), 3.31$ (s, 3H), 3.15 (t, J = 6.4 Hz, 2H, H9/H15), $2.86-2.61(\mathrm{~m}, 4 \mathrm{H}, \mathrm{H} 7$ and $\mathrm{H} 13), 2.02-1.84(\mathrm{~m}, 2 \mathrm{H}, \mathrm{H} 8 / \mathrm{H} 14), 1.79$ (s, 5H, H8/H14 and $\left.\mathrm{NHCOCH}_{3}\right), 1.43\left(\mathrm{~s}, 9 \mathrm{H}, \mathrm{OC}\left(\mathrm{CH}_{3}\right)_{3}\right) .{ }^{13} \mathrm{C} \mathrm{NMR}\left(101 \mathrm{MHz}, d_{3}-\mathrm{MeOD}\right) \delta_{\mathrm{C}} 173.4\left(\mathrm{NHCOCH}_{3}\right), 158.6(\mathrm{NHCOOC}), 146.4(\mathrm{C} 11), 123.7(\mathrm{C} 10)$, 88.2 (C1'), 87.6 (C1), 86.7 (C1"), 83.7, 80.4, 80.3, 79.9, 79.0, 75.6, 75.0, 73.8, 73.2, 72.4, 70.6, 68.9, 67.4, 63.5 (C12), 62.8, 62.7, 61.9, 57.2 (C2'), 40.2 (C9/C15), 31.5 (C8/C14), 31.2 (C8/C14), $28.9(\mathrm{C} 7 / \mathrm{C} 13) 28.8\left(\mathrm{OC}\left(\mathrm{CH}_{3}\right)_{3}\right), 28.1(\mathrm{C} 7 / \mathrm{C} 13), 22.6(\mathrm{NHCOCH}) . \mathrm{HRMS}$ (ES): Calcd. for $\mathrm{C}_{34} \mathrm{H}_{59} \mathrm{~N}_{5} \mathrm{O}_{17} \mathrm{~S}_{2} \mathrm{H}: 874.3426$ : found. 874.3424.

1-(tButyl(3-mercaptopropyl)carbamate) 3-O-[(2'-acetamido-2'-deoxy -4'-O-[(2"-acetamido-2"-deoxy -1"-thio- $\beta$-Dglucopyranosyl)propyl]- $\beta$-D-glucopyranosyl)-1H-[1,2,3]-triazol-4-yl-methyl]- $\beta$-D-galactopyranoside S53

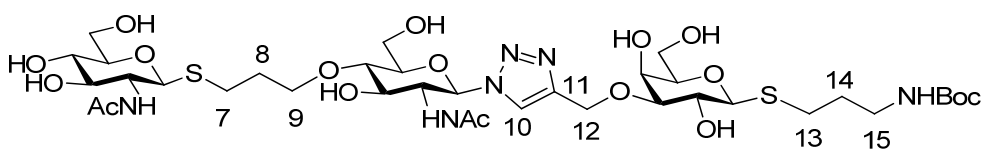

The product S53 was synthesized according to general procedure A and obtained as light yellow oil $(0.0106 \mathrm{~g} ; 0.0116 \mathrm{mmol})$ in $68 \%$ yield. $R_{\mathrm{f}} 0.65$ (ethyl acetate/methanol 1:1). [ $\left.\alpha\right]_{\mathrm{D}}{ }^{295 \mathrm{~K}}-7.0(c \mathrm{1}, \mathrm{MeOH}) .{ }^{1} \mathrm{H}$ NMR $\left(400 \mathrm{MHz}, d_{3}-\mathrm{MeOD}\right) \delta_{\mathrm{H}} 8.22(\mathrm{~s}, 1 \mathrm{H}, \mathrm{H} 10), 5.74(\mathrm{~d}, \mathrm{~J}=$ $9.7 \mathrm{~Hz}, 1 \mathrm{H}, \mathrm{H} 1$ '), 4.84 (d, J = 12.6 Hz, 1H, H12a), 4.78 (d, J = $12.6 \mathrm{~Hz}, 1 \mathrm{H}, \mathrm{H} 12 \mathrm{~b}), 4.50$ (d, J = 10.2 Hz, 1H, H1/H1"), 4.36 (d, J = 9.6 $\mathrm{Hz}, 1 \mathrm{H}, \mathrm{H} 1 / \mathrm{H} 1 "), 4.19(\mathrm{t}, \mathrm{J}=10.0 \mathrm{~Hz}, 1 \mathrm{H}), 4.11-3.95(\mathrm{~m}, 3 \mathrm{H}), 3.88(\mathrm{~d}, \mathrm{~J}=11.6 \mathrm{~Hz}, 3 \mathrm{H}), 3.84-3.49(\mathrm{~m}, 12 \mathrm{H}, \mathrm{H} 2, \mathrm{H} 2 \mathrm{\prime}$ and H2"), 3.49 - $3.34(\mathrm{~m}, 4 \mathrm{H}), 3.15(\mathrm{t}, \mathrm{J}=6.6 \mathrm{~Hz}, 2 \mathrm{H}, \mathrm{H} 9 / \mathrm{H} 15), 2.96-2.58(\mathrm{~m}, 4 \mathrm{H}, \mathrm{H} 7$ and H13), $1.99(\mathrm{~s}, 3 \mathrm{H}, \mathrm{NHCOCH}), 1.95-1.83(\mathrm{~m}, 4 \mathrm{H}, \mathrm{H} 8$ and $\mathrm{H} 14), 1.79\left(, 3 \mathrm{H}, \mathrm{NHCOCH}_{3}\right), 1.43\left(\mathrm{~s}, 9 \mathrm{H}, \mathrm{s}, 9 \mathrm{H}, \mathrm{OC}\left(\mathrm{CH}_{3}\right)_{3}\right) .{ }^{13} \mathrm{C} \mathrm{NMR}\left(101 \mathrm{MHz}, d_{3}-\mathrm{MeOD}\right) \delta_{\mathrm{c}} 173.6\left(\mathrm{NHCOCH}_{3}\right), 173.5$ $\left(\mathrm{NHCOCH}_{3}\right), 158.6$ (NHCOOC), 146.2 (C11), 121.5 (C10), 88.2 (C1'), 87.6 (C1/C1"), 85.7 (C1/C1"), 83.7, 82.2, 80.4, 80.4, 79.9, 79.1, 77.5, 75.6, 72.3, 72.1, 70.6, 67.5, 63.5 (1C, C12), 63.0, 62.7, 61.9, 57.2, 56.3, 40.2 (C9/C15), 31.4 (C8/C14), 31.3 (C8/C14), 28.8 $\left(\mathrm{OC}\left(\mathrm{CH}_{3}\right)_{3}\right), 28.1(\mathrm{C} 7 / \mathrm{C} 13), 27.6(\mathrm{C} 7 / \mathrm{C} 13) .23 .0\left(\mathrm{NHCOCH}_{3}\right), 22.6\left(\mathrm{NHCOCH}_{3}\right)$. HRMS (ES): Calcd. for $\mathrm{C}_{36} \mathrm{H}_{62} \mathrm{~N}_{6} \mathrm{O}_{17} \mathrm{~S}_{2} \mathrm{Na}$ : 937.3511; found 937.3512 . 


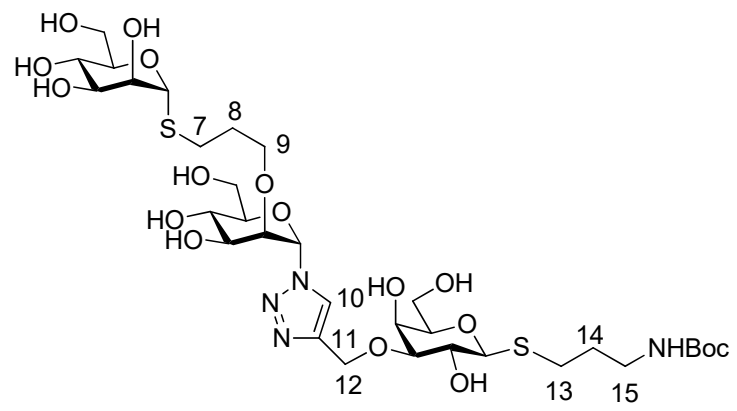

The product S54 was synthesized according to general procedure A and obtained as yellow oil $(0.0135 \mathrm{~g} ; 0.0162 \mathrm{mmol})$ in quantitative yield. $R_{\mathrm{f}} 0.66$ (ethyl acetate/methanol 2:1.5). [ $\left.\alpha\right]_{\mathrm{D}}{ }^{295 \mathrm{~K}}+34.0(c 0.5, \mathrm{MeOH}) .{ }^{1} \mathrm{H}$ NMR $\left(400 \mathrm{MHz}, d_{3}-\mathrm{MeOD}\right) \delta_{\mathrm{H}} 8.21(\mathrm{~s}, 1 \mathrm{H}, \mathrm{H} 10), 6.14(\mathrm{~s}$, $\left.1 \mathrm{H}, \mathrm{H} 1^{\prime}\right), 5.24\left(\mathrm{~s}, 1 \mathrm{H}, \mathrm{H} 1{ }^{\prime}\right), 4.94-4.78(\mathrm{~m}, 2 \mathrm{H}, \mathrm{H} 12), 4.43\left(\mathrm{~d}, \mathrm{~J}=2.8 \mathrm{~Hz}, 1 \mathrm{H}, \mathrm{H} 2^{\prime}\right), 4.34(\mathrm{~d}, \mathrm{~J}=9.7 \mathrm{~Hz}, 1 \mathrm{H}, \mathrm{H} 1), 4.13-4.05(\mathrm{~m}, 4 \mathrm{H}$, $\left.\mathrm{H}^{\prime}\right), 3.94-3.59(\mathrm{~m}, 11 \mathrm{H}, \mathrm{H} 2$ and H2"), $3.52(\mathrm{t}, \mathrm{J}=5.8 \mathrm{~Hz}, 2 \mathrm{H}), 3.44-3.36(\mathrm{~m}, 2 \mathrm{H}), 3.15(\mathrm{t}, \mathrm{J}=6.6 \mathrm{~Hz}, 3 \mathrm{H}, \mathrm{H9} / \mathrm{H} 15), 2.84-2.63(\mathrm{~m}$, $4 \mathrm{H}, \mathrm{H} 9$ and $\mathrm{H} 13), 2.02-1.85$ (m, 2H, H8/H14), $1.82-1.74(\mathrm{~m}, 2 \mathrm{H}, \mathrm{H} 8 / \mathrm{H} 14), 1.43\left(\mathrm{~s}, 9 \mathrm{H}, \mathrm{OC}\left(\mathrm{CH}_{3}\right)_{3}\right) .{ }^{13} \mathrm{C} \mathrm{NMR}\left(101 \mathrm{MHz}, d_{3}-\mathrm{MeOD}\right)$ $\delta_{\mathrm{C}} 158.6$ (NHCOOC), 146.7 (C11), 125.1 (C10), 87.6 (C1), 86.8 (C1"), 85.8 (C1'), 84.1, 80.4, 79.9, 78.8, 78.5 (C2'), 75.1, 73.8, 73.2, 72.1, 70.7, 70.5, 68.9, 67.3, 63.6 (C12), 62.8, 62.6, 62.5, 40.2 (C9/C15), 31.3 (C8/C14), 30.8 (C8/C14), $28.8\left(\mathrm{OC}(\mathrm{CH})_{3}\right), 28.1(\mathrm{C} 7$ and C13). HRMS (ES): Calcd. for $\mathrm{C}_{32} \mathrm{H}_{56} \mathrm{~N}_{4} \mathrm{O}_{17} \mathrm{~S}_{2} \mathrm{H}$ : 833.3160; found. 833.3159.

1-(tButyl(3-mercaptopropyl)carbamate) 3-O-[(3'-O-[(3"-O-[(1"'-thio- $\beta$-D-galactopyranosyl)propyl]- $\beta$-Dgalactopyranosyl)-1H-[1,2,3]-triazol-4-yl-methyl]-1'-thio- $\beta$-D-galactopyranosyl)propyl]- $\beta$-D-galactopyranoside S55

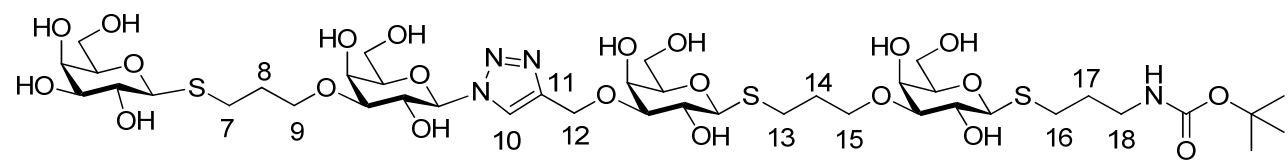

The product S55 was synthesized according to general procedure A and obtained as light orange foam (0.0224 g; $0.0209 \mathrm{mmol})$ in $96 \%$ yield. $R_{\mathrm{f}} 0.46$ (ethyl acetate/methanol 2:1.5). $[\alpha]_{\mathrm{D}}{ }^{295 \mathrm{~K}}+18.2(c 1, \mathrm{MeOH}) .{ }^{1} \mathrm{H} \mathrm{NMR}\left(400 \mathrm{MHz}, d_{3}-\mathrm{MeOD}\right) \delta_{\mathrm{H}} 5.57(\mathrm{~d}, J=9.2 \mathrm{~Hz}, 1 \mathrm{H}$, H1"), 4.83 (d, J = 12.5 Hz, 1H, H12a), 4.76 (d, J = 12.5 Hz, 1H, H12b), $4.37-4.26$ (m, 3H, H1, H1' and H1'"), $4.23-4.13$ (m, 2H, H2"), $4.06(\mathrm{dd}, \mathrm{J}=7.8,2.8 \mathrm{~Hz}, 2 \mathrm{H}), 3.89-3.76(\mathrm{~m}, 4 \mathrm{H}), 3.75-3.58(\mathrm{~m}, 8 \mathrm{H}), 3.57-3.40(\mathrm{~m}, 6 \mathrm{H}), 3.38(\mathrm{dd}, \mathrm{J}=9.2,3.1 \mathrm{~Hz}, 1 \mathrm{H}), 3.27(\mathrm{dt}, \mathrm{J}$ $=3.3,1.6 \mathrm{~Hz}, 5 \mathrm{H}), 3.21(\mathrm{dd}, \mathrm{J}=9.2,3.1 \mathrm{~Hz}, 1 \mathrm{H}), 3.11(\mathrm{t}, \mathrm{J}=6.7 \mathrm{~Hz}, 2 \mathrm{H}, \mathrm{H} 9 / \mathrm{H} 15 / \mathrm{H} 18), 2.96-2.57(\mathrm{~m}, 6 \mathrm{H}, \mathrm{H} 7, \mathrm{H} 13$ and H16), $2.03-$ $1.86(\mathrm{~m}, 4 \mathrm{H}, \mathrm{H} 8 / \mathrm{H} 14 / \mathrm{H} 17), 1.82-1.67(\mathrm{~m}, 2 \mathrm{H}, \mathrm{H} 8 / \mathrm{H} 14 / \mathrm{H} 17), 1.39\left(\mathrm{~s}, 9 \mathrm{H}, \mathrm{OC}\left(\mathrm{CH}_{3}\right)_{3}\right) .{ }^{13} \mathrm{C} \mathrm{NMR}\left(101 \mathrm{MHz}, d_{3}-\mathrm{MeOD}\right) \delta_{\mathrm{C}} 158.6$ (NHCOOC), 146.4 (C11), 90.2 (C1"), 87.8, 87.7 (C1, C1' and C1"'), 84.3, 80.7, 80.5, 79.8, 76.3, 71.4, 70.6, 70.5, 69.1, 67.3, 67.0, 63.5 (C12), 62.8, 62.4, 40.2 (C9/C15/C18), 31.3 (C8/C14/C17), $28.8\left(\mathrm{OC}\left(\mathrm{CH}_{3}\right)_{3}\right), 28.1$ (C7/C13/C16), 27.7 (C7/C13/C16), 27.6 (C7/C13/C16). HRMS (ES): Calcd. for $\mathrm{C}_{41} \mathrm{H}_{72} \mathrm{~N}_{4} \mathrm{O}_{22} \mathrm{~S}_{3} \mathrm{H}$ : 1069.3879 ; found. 1069.3888.

1-(tButyl(3-mercaptopropyl)carbamate) 3-O-[(-3'-O-[(-3"-O-[(- $\beta-D-g a l a c t o p y r a n o s y l)-1 \mathrm{H}-[1,2,3]-$ triazol-4-yl-methyl]-1"thio- $\beta$-D-galactopyranosyl)propyl]- $\beta$-D-galactopyranosyl)-1H-[1,2,3]-triazol-4-yl-methyl]- $\beta$-D-galactopyranoside S56

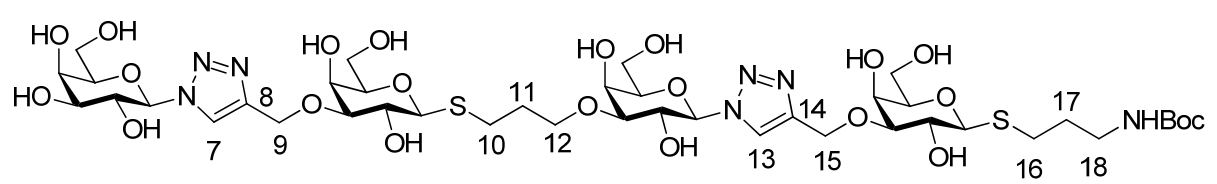

The product S56 was synthesized according to general procedure A and obtained as light brown oil $(0.243 \mathrm{~g} ; 0.0226 \mathrm{mmol})$ in quantitative yield. $R_{\mathrm{f}} 0.26$ (ethyl acetate/methanol 1:1). [ $\left.\alpha\right]_{\mathrm{D}}{ }^{295 \mathrm{~K}}-47.4(c 1, \mathrm{MeOH}) .{ }^{1} \mathrm{H}$ NMR $\left(400 \mathrm{MHz}, d_{3}-\mathrm{MeOD}\right) \delta_{\mathrm{H}} 8.25(\mathrm{~s}, 2 \mathrm{H}, \mathrm{H} 7$ and $\mathrm{H} 13$ ), 5.57 (d, J = 9.2 Hz, 2H, H1' and H1'"), 4.87 (d, $\mathrm{J}=12.3 \mathrm{~Hz}, 2 \mathrm{H}, \mathrm{H} 9 \mathrm{a}$ and H15a), 4.81 (d, J = 12.3 Hz, 2H, H9b and H15b), 4.34 (d, $\mathrm{J}=9.7 \mathrm{~Hz}, 2 \mathrm{H}, \mathrm{H} 1$ and $\mathrm{H} 1 "), 4.28-4.06(\mathrm{~m}, 4 \mathrm{H}, \mathrm{H} 2$ ' and H2"'), 3.99 (d, J = 2.5 Hz, 2H), $3.88-3.62(\mathrm{~m}, 15 \mathrm{H}, \mathrm{H} 2 \mathrm{and} \mathrm{H} 2 ")$, $3.60-3.47(\mathrm{~m}, 5 \mathrm{H}), 3.47-3.37(\mathrm{~m}, 1 \mathrm{H}), 3.16(\mathrm{t}, \mathrm{J}=6.7 \mathrm{~Hz}, 2 \mathrm{H}, \mathrm{H} 12 / \mathrm{H} 18), 2.99-2.55(\mathrm{~m}, 4 \mathrm{H}, \mathrm{H} 10$ and H16), $2.06-1.92(\mathrm{~m}, 2 \mathrm{H}$, 
$\mathrm{H} 11 / \mathrm{H} 17), 1.87$ - 1.71 (m, 2H, H11/H17), 1.44 (s, 9H, OC( $\left.\left.\mathrm{CH}_{3}\right)_{3}\right) .{ }^{13} \mathrm{C} \mathrm{NMR}\left(101 \mathrm{MHz}, d_{3}-\mathrm{MeOD}\right) \delta_{\mathrm{c}} 158.6$ (NHCOOC), 146.6 (C8/C14), 146.5 (C8/C14), 123.8 (C7/C13), 123.8 (C7/C13), 90.3 (C1' and C1"), 87.6 (C1 and C1"), 84.0, 80.5, 79.9, 75.3, 71.5, 70.5, 70.3, 67.3, 63.6 (C19 and C15), 62.7, 62.4, 40.2 (C2/C8), 31.3 (C10 and $\mathrm{C} 16), 28.8\left(\mathrm{OC}\left(\mathrm{CH}_{3}\right)_{3}\right), 28.1$ (C11 and C17). HRMS (ES): Calcd. for $\mathrm{C}_{41} \mathrm{H}_{69} \mathrm{~N}_{7} \mathrm{O}_{22} \mathrm{~S}_{2} \mathrm{H}: 1076.4015$; found. 1076.4019 .

1-(tButyl(3-mercaptopropyl)carbamate) 3-O-[(2'-acetamido-2'-deoxy-4'-O- [(3"-O-[(- $\alpha$-D-mannopyranosyl)-1H-[1,2,3]-triazol4-yl-methyl]-1"-thio- $\beta$-D-galactopyranosyl)propyl]- $\beta$-D-glucopyranosyl)-1H-[1,2,3]-triazol-4-yl-methyl]- $\beta$-Dgalactopyranoside S57

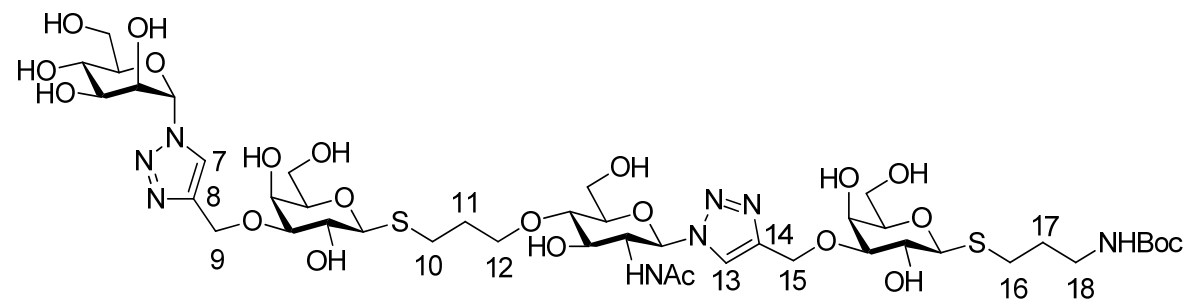

The product S57 was synthesized according to general procedure A and obtained as light brown foam $(0.0156 \mathrm{~g} ; 0.0140 \mathrm{mmol})$ in $58 \%$ yield. $R_{\mathrm{f}} 0.68$ (ethyl acetate/methanol 2:1). [ $\left.\alpha\right]_{\mathrm{D}}{ }^{295 \mathrm{~K}}-6.2\left(c\right.$ 1, MeOH). ${ }^{1} \mathrm{H}$ NMR $\left(400 \mathrm{MHz}, d_{3}-\mathrm{MeOD}\right) \delta_{\mathrm{H}} 8.22(\mathrm{~s}, 1 \mathrm{H}, \mathrm{H} 7 / \mathrm{H} 13), 8.20(\mathrm{~s}$, $1 \mathrm{H}, \mathrm{H} 7 / \mathrm{H} 13), 6.04$ (d, J = 2.7 Hz, 1H, H1'/H1'"), 5.74 (d, J = 9.8 Hz, 1H, H1'/H1'"), 5.29 (d, J = 17.2 Hz, 1H), 5.15 (d, J = $10.3 \mathrm{~Hz}, 1 \mathrm{H}$ ), $4.84(\mathrm{~d}, \mathrm{~J}=12.7 \mathrm{~Hz}, 2 \mathrm{H}, \mathrm{H} 9 \mathrm{a}$ and $\mathrm{H} 15 \mathrm{a}), 4.78(\mathrm{~d}, \mathrm{~J}=12.7 \mathrm{~Hz}, 2 \mathrm{H}, \mathrm{H} 9 \mathrm{~b}$ and H15b), $4.69(\mathrm{t}, \mathrm{J}=6.1 \mathrm{~Hz}, 1 \mathrm{H}), 4.51-4.42(\mathrm{~m}, 1 \mathrm{H}), 4.36$ (dd, $J=9.7,2.7 \mathrm{~Hz}, 1 \mathrm{H}, \mathrm{H} 1$ and H1"), $4.29-4.12(\mathrm{~m}, 2 \mathrm{H}), 4.15-3.95(\mathrm{~m}, 3 \mathrm{H}), 3.93-3.35(\mathrm{~m}, 21 \mathrm{H}), 3.15(\mathrm{t}, \mathrm{J}=6.7 \mathrm{~Hz}, 2 \mathrm{H}, \mathrm{H} 12 / \mathrm{H} 18)$, $2.97-2.61(\mathrm{~m}, 4 \mathrm{H}, \mathrm{H} 10$ and $\mathrm{H} 16), 2.05-1.83(\mathrm{~m}, 4 \mathrm{H}, \mathrm{H} 11$ and $\mathrm{H} 17), 1.79(\mathrm{~s}, 3 \mathrm{H}, \mathrm{NHCOCH}), 1.43\left(\mathrm{~s}, 9 \mathrm{H}, \mathrm{s}, 9 \mathrm{H}, \mathrm{OC}(\mathrm{CH})_{3}\right) .{ }^{13} \mathrm{C} \mathrm{NMR}$ (101 MHz, d $\left.d_{3}-\mathrm{MeOD}\right) \delta_{\mathrm{C}} 173.5\left(\mathrm{NHCOCH}_{3}\right), 158.6$ (NHCOOC), 146.2 (C8/C14), 136.4 (C8/C14), 130.3 (C7/C13), 128.7 (C7/C13), 116.9, 88.7 (C1'/C1"'), 88.3 (C1'/C1'"), 87.6 (C1 and C1"), 83.7, 80.4, 80.3, 79.9, 78.8, 78.6, 75.7, 74.9, 72.8, 70.6, 70.3, 68.9, 67.5, 63.5, 62.7, 61.9, 57.2, 40.2 (C12/C18), 31.7 (C11/C17), 31.3 (C11/C17),), $\left.28.8\left(\mathrm{OC}_{(\mathrm{CH}}\right)_{3}\right), 28.1$ (C10/C16), 27.5 (C10/C16), 22.6 $\left(\mathrm{NHCOCH}_{3}\right)$. HRMS (ES): Calcd. for $\mathrm{C}_{43} \mathrm{H}_{72} \mathrm{~N}_{8} \mathrm{O}_{22} \mathrm{~S}_{2} \mathrm{Na}$ : 1139.4100 ; found 1139.4115 .

\section{Synthesis of oligosaccharide mimic S34 from deprotected building blocks}

1-(tButyl(3-mercaptopropyl)carbamate) 2,4,6-tri-O-acetyl-3-O-[(2',4',6'-tri-O-acetyl-3'-O-[(2",3",4",6"-tetra-O-acetyl-1"thio- $\beta$-D-galactopyranosyl)propyl]- $\beta$-D-galactopyranosyl)-1H-[1,2,3]-triazol-4-yl-methyl]- $\beta$-D-galactopyranoside S34

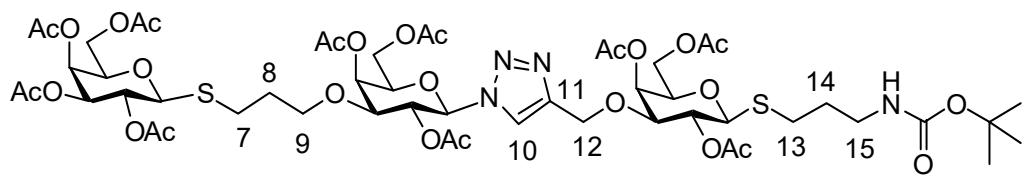

Vazo44 (5.1 mg; $15.7 \mu \mathrm{mol} ; 0.1$ equiv.) was added to a solution of deprotected thiolate dA2 (0.0223 g; $0.102 \mathrm{mmol} ; 1.3$ equiv.) and deprotected azide alkene dB1 (0.0192 g; $0.0783 \mathrm{mmol} ; 1$ equiv.) dissolved degassed acetate buffer (0.9 mL; $0.05 \mathrm{~mol} / \mathrm{L} ; \mathrm{pH} 4) \mathrm{under}$ a $\mathrm{N}_{2}$ atm. The mixture was irradiated at $360 \mathrm{~nm}$ overnight. When the reaction had run to completion $\mathrm{pH}$ was adjusted to $8 \mathrm{using}$ degassed $2 \mathrm{M} \mathrm{NaOH}$ (aq) before the deprotected thioether alkyne dD1 (0.0490 g; $0.125 \mathrm{mmol} ; 1.6$ equiv.), degassed $1 \mathrm{M}$ sodium ascorbate (aq) $\left(7.8 \mu \mathrm{L} ; 15.5 \mu \mathrm{mol} ; 0.1\right.$ equiv.) and $1 \mathrm{M} \mathrm{CuSO}_{4}$ (aq) $(3.8 \mu \mathrm{L} ; 6.24 \mu \mathrm{mol} ; 0.05$ equiv.) were added and the mixture was heated to $70{ }^{\circ} \mathrm{C}$ overnight. When the reaction had run to completion the solvents were evaporated and the residue was redissolved in acetic acid $(2 \mathrm{~mL})$ and dry pyridine $(2 \mathrm{~mL})$ under a $\mathrm{N}_{2}$ atm, and left overnight. The solvents were evaporated followed by co-evaporation with toluene three times, before the residue was purified by column chromatography (ethyl acetate/pentane 2:1 to ethyl acetate) and obtained as clear oil in $43 \%$ yield $(42.4 \mathrm{mg} ; 0.0338 \mathrm{mmol})$ in three steps. 


\section{NMR spectra}

\section{Group A building blocks}

2,3,4,6-tetra-O-acetyl-a-D-galactopyranosyl bromide $\mathbf{S 2}$ 


$$
\text { (OcO) }
$$

S2
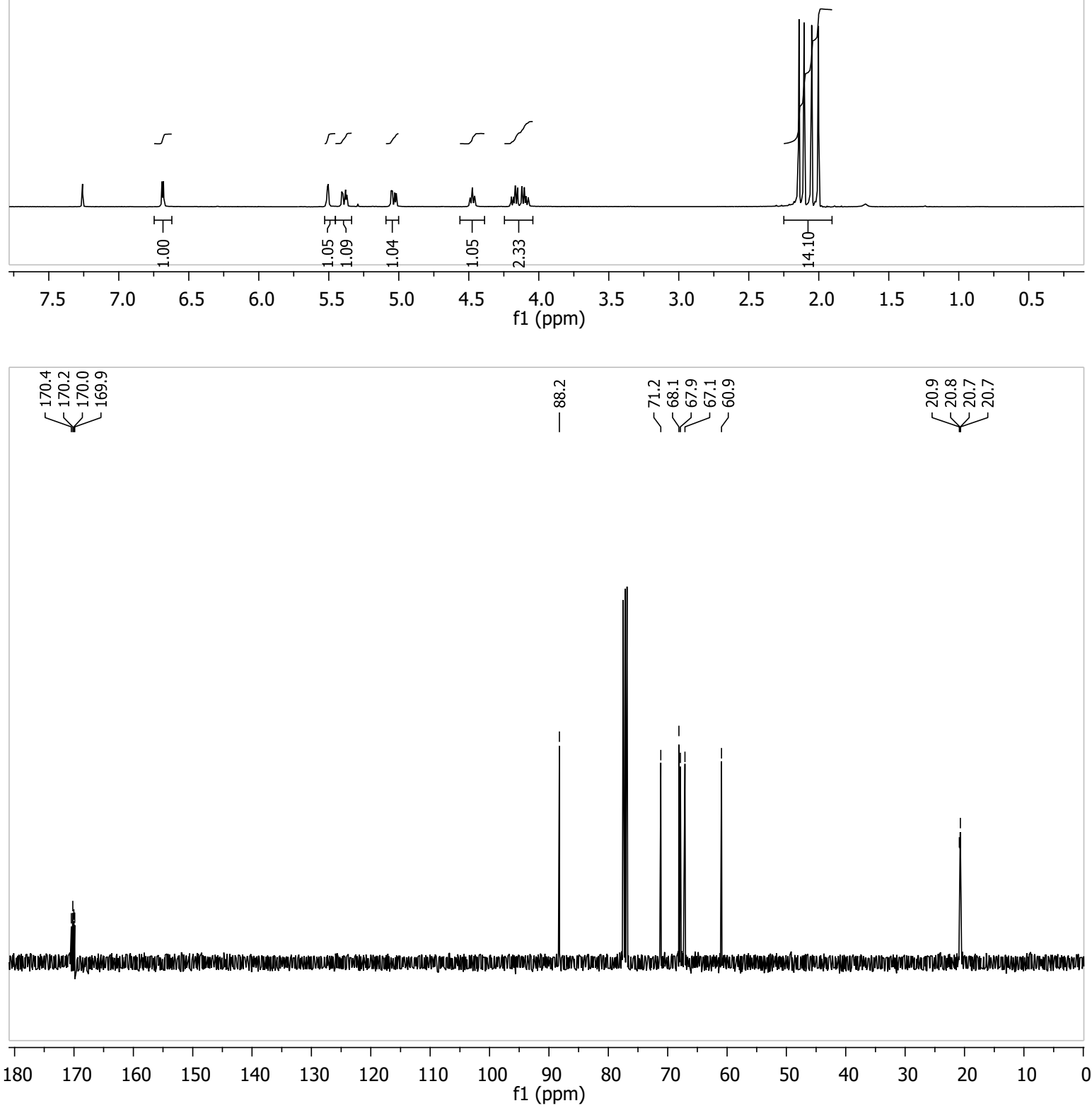

2,3,4,6-tetra-O-acetyl- $\beta$-D-galactopyranosyl azide A1 

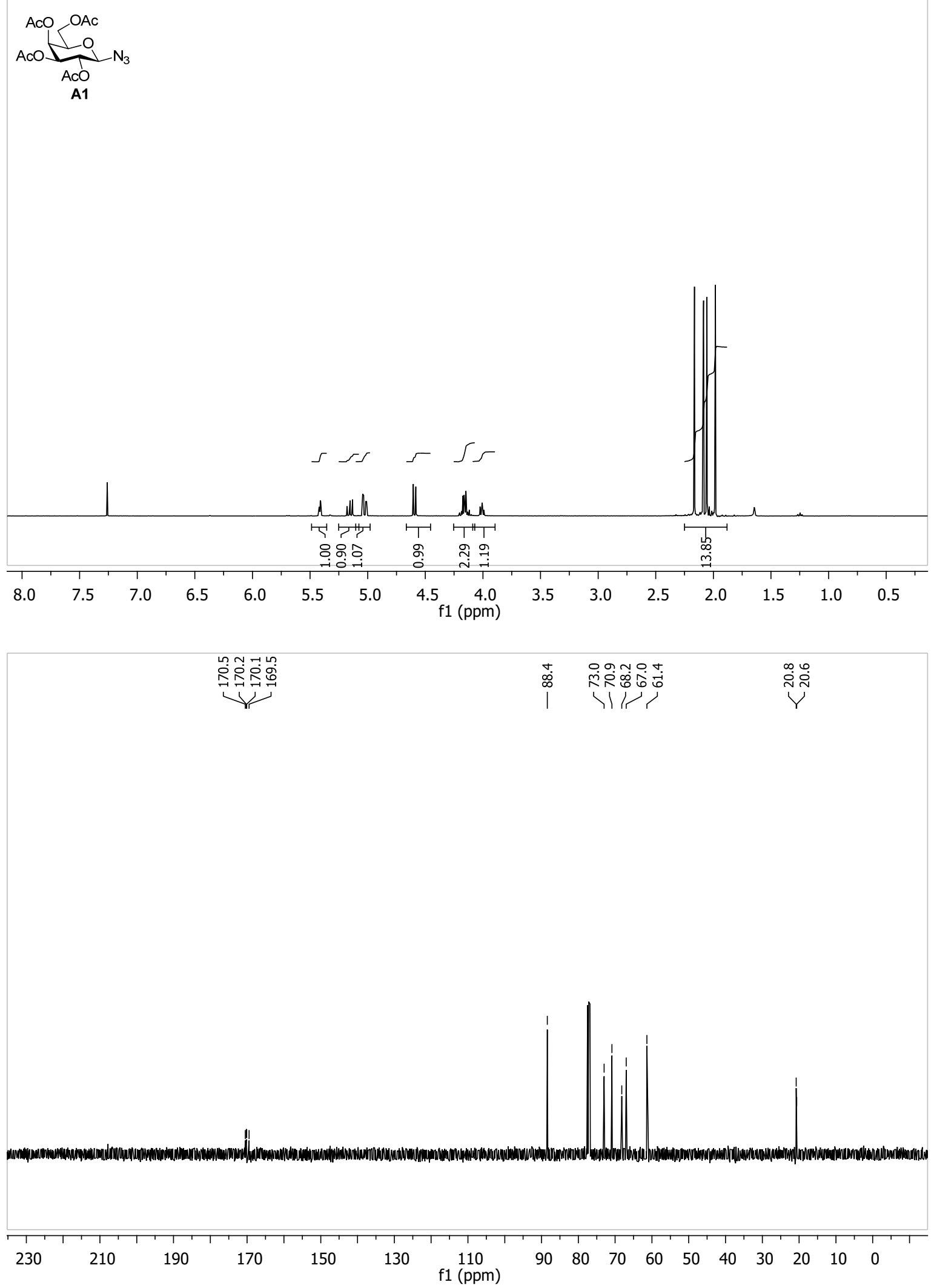

2,3,4,6-Tetra-O-acetyl- $\beta$-D-galactopyranosyl-1-isothiouronium bromide S3 

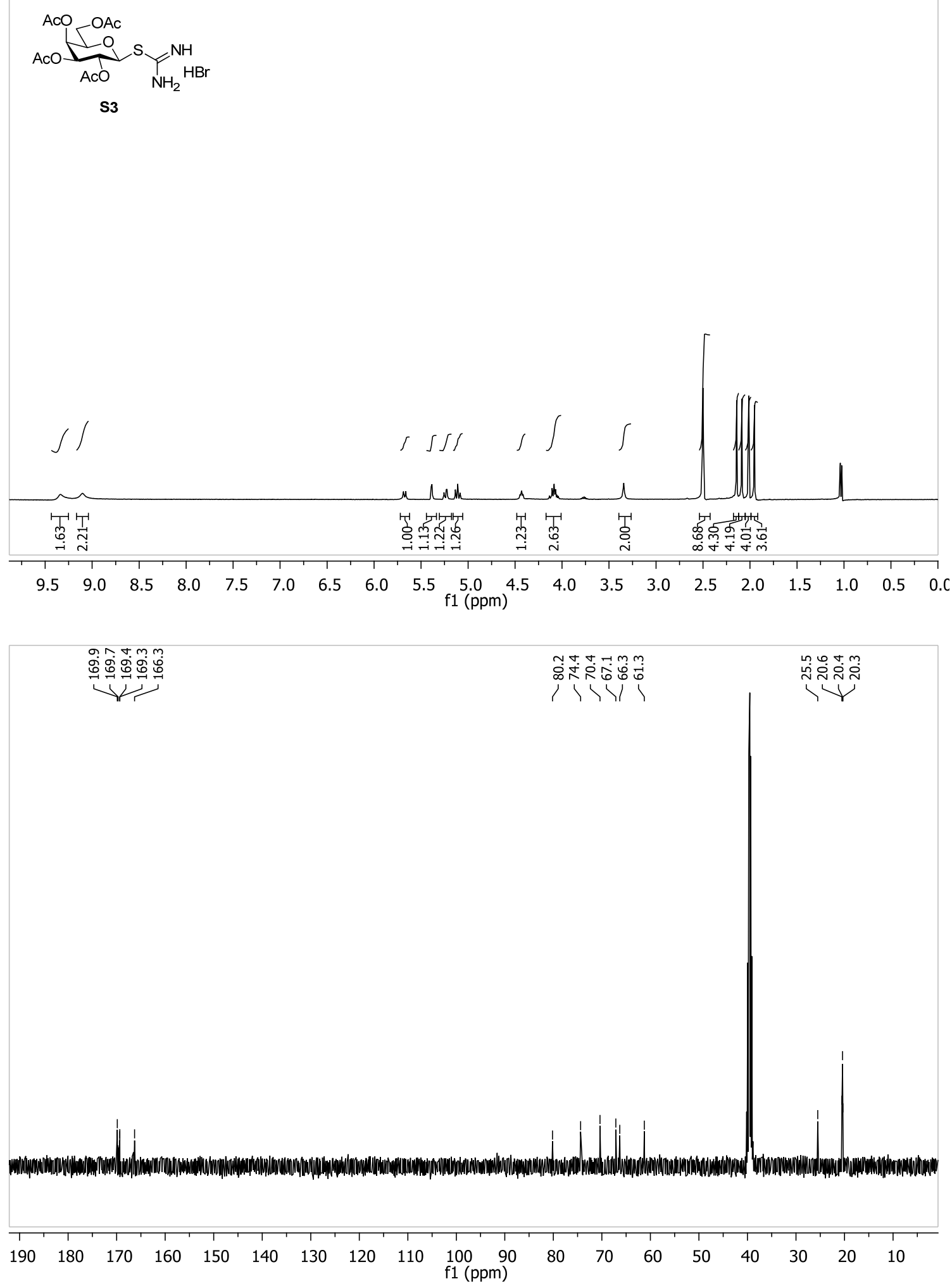

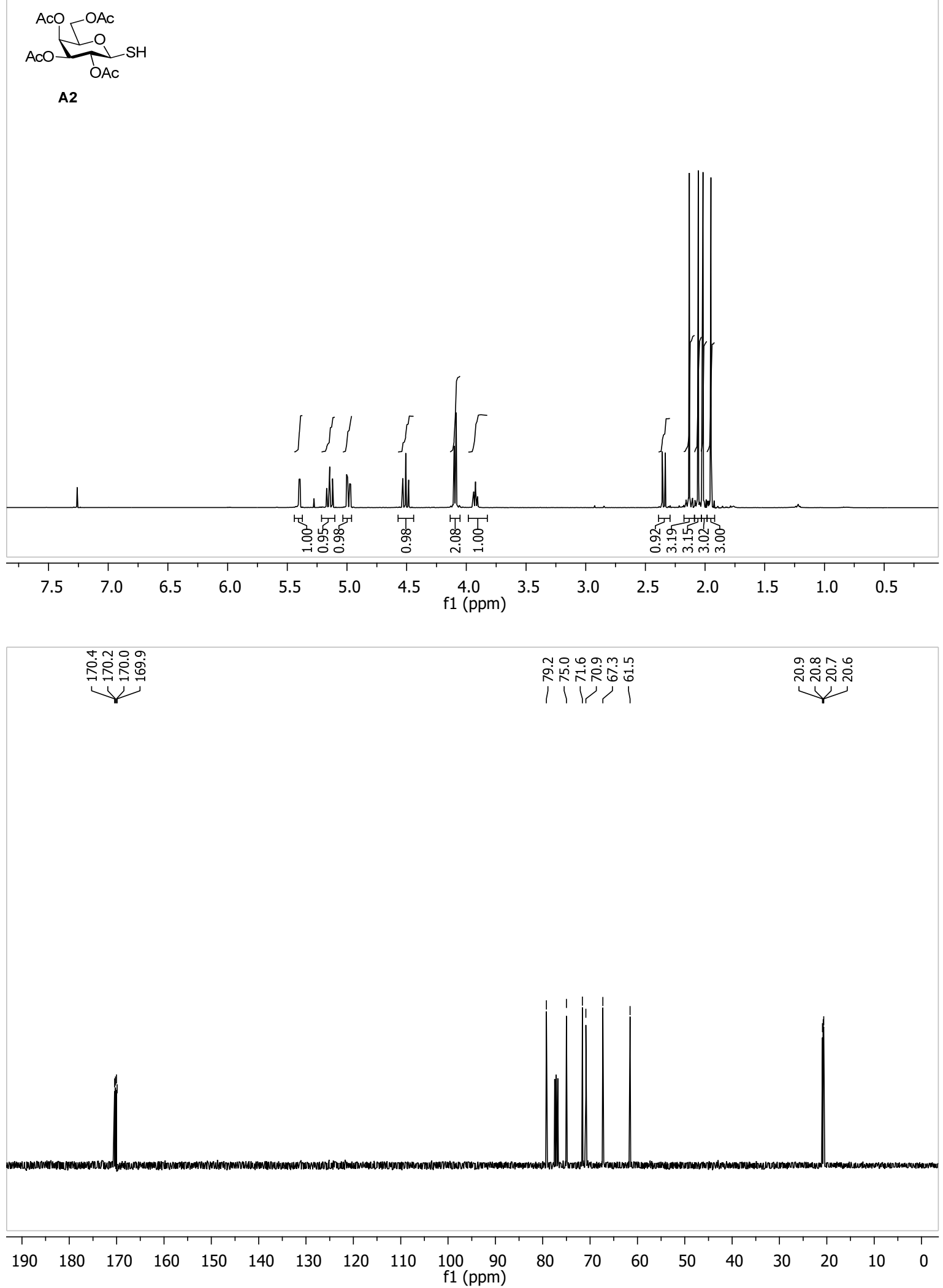


\section{1,2,3,4,6-Penta-O-acetyl- $\alpha$-D-mannopyranose S5}
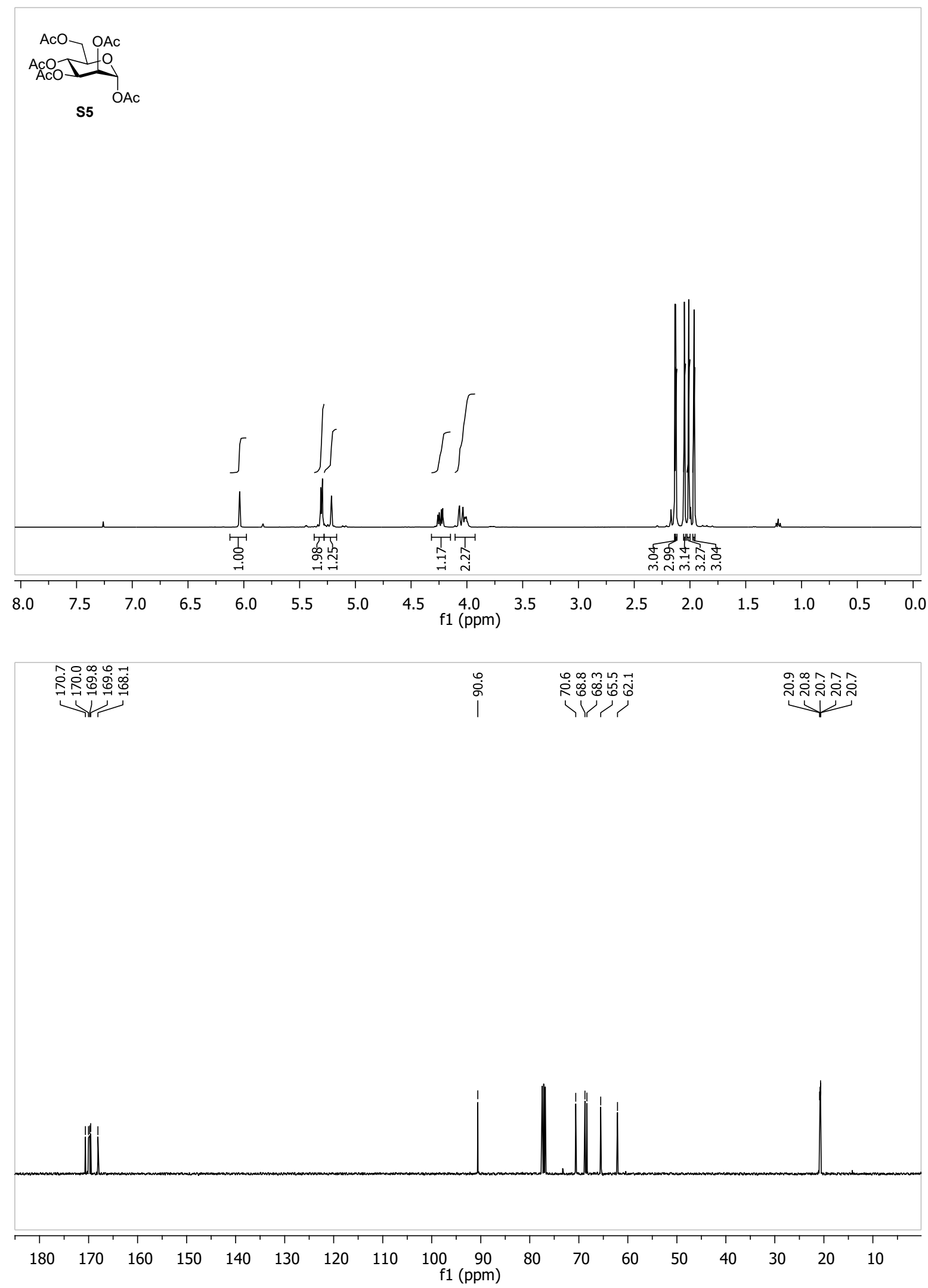


\section{2,3,4,6-Tetra-O-acetyl- $\alpha$-D-mannopyranosyl azide A3}
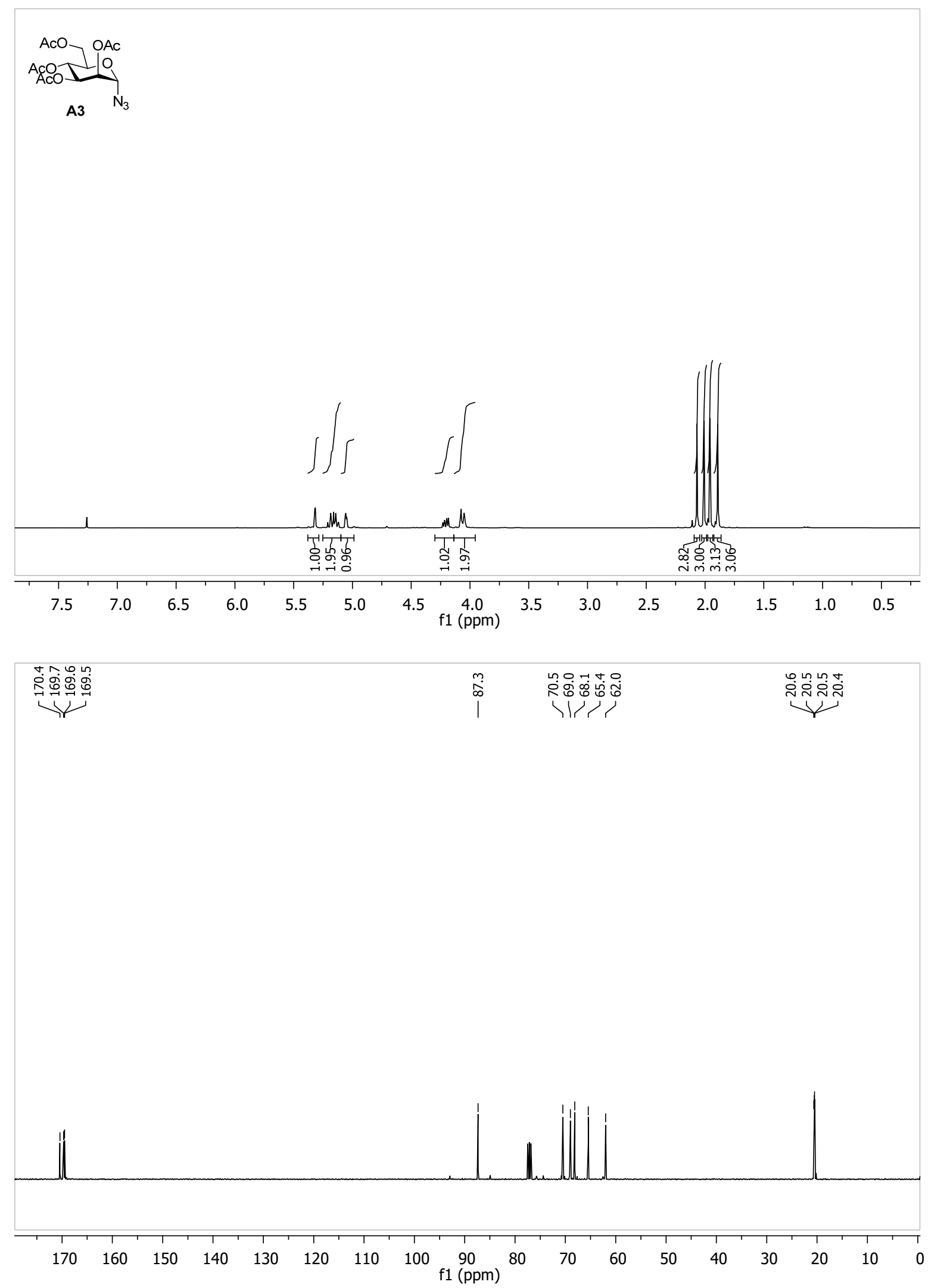

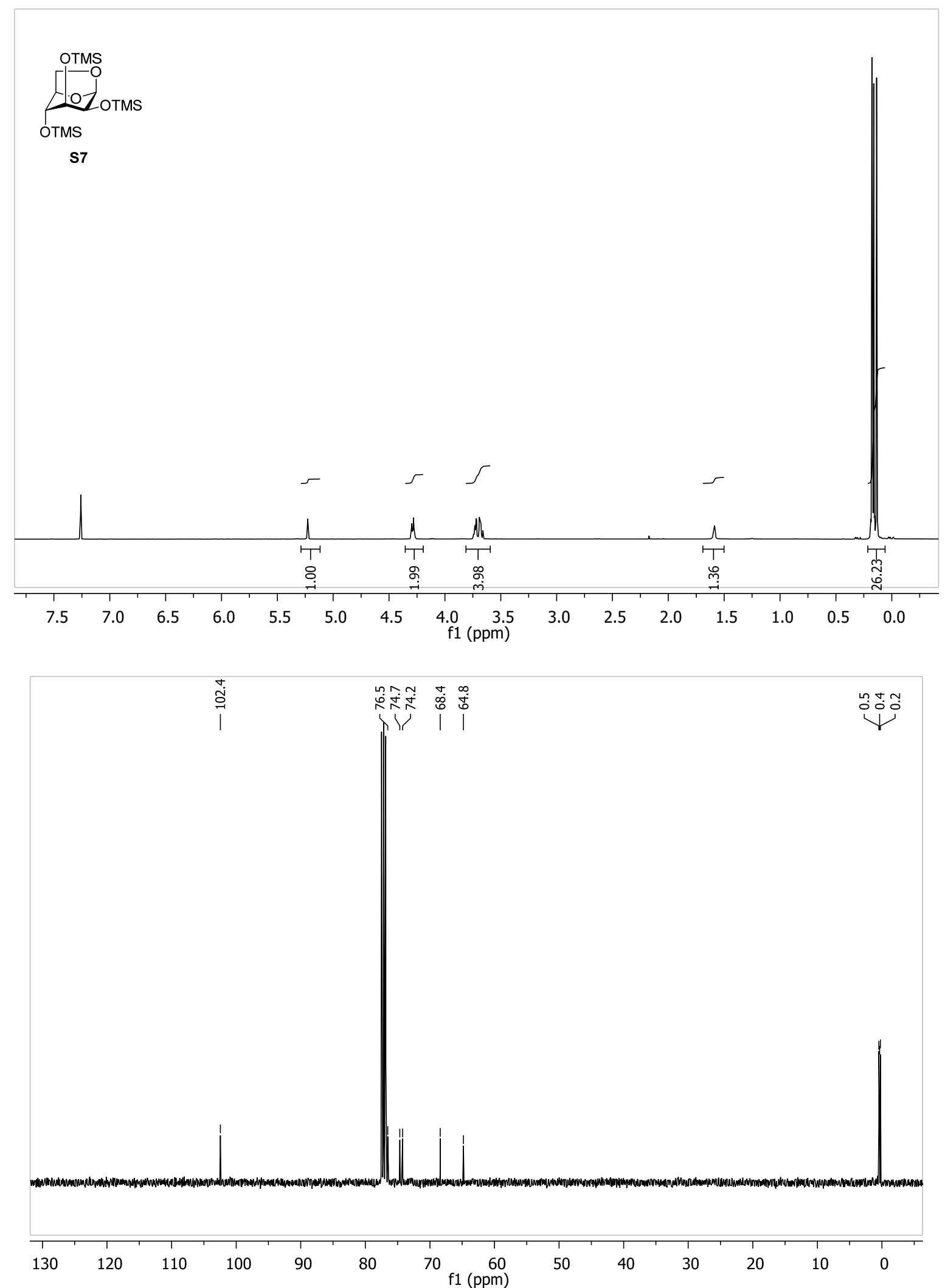

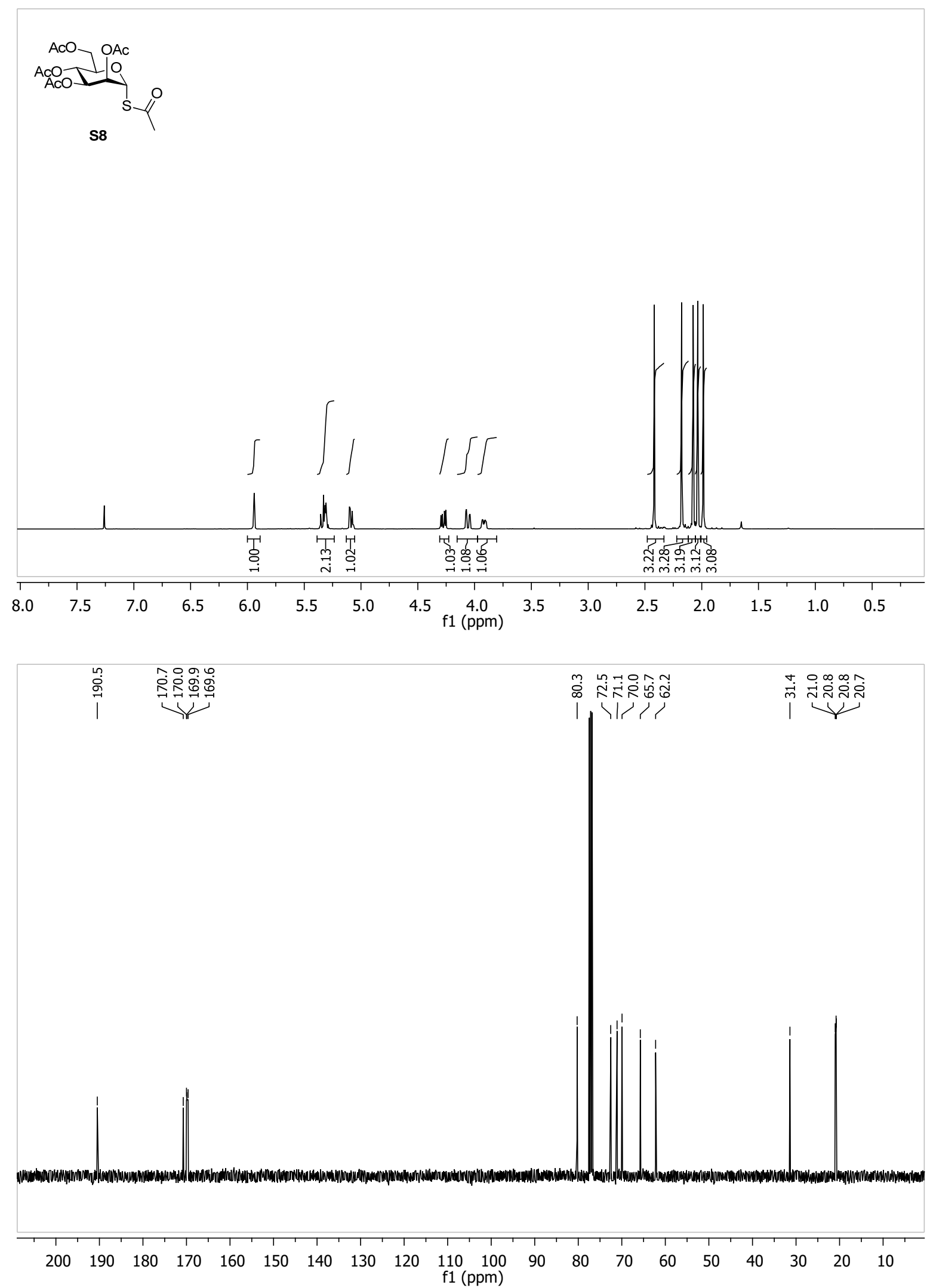


\section{2,3,4,6-Tetra-O-acetyl- $\alpha$-D-mannopyranosyl thiol A4}
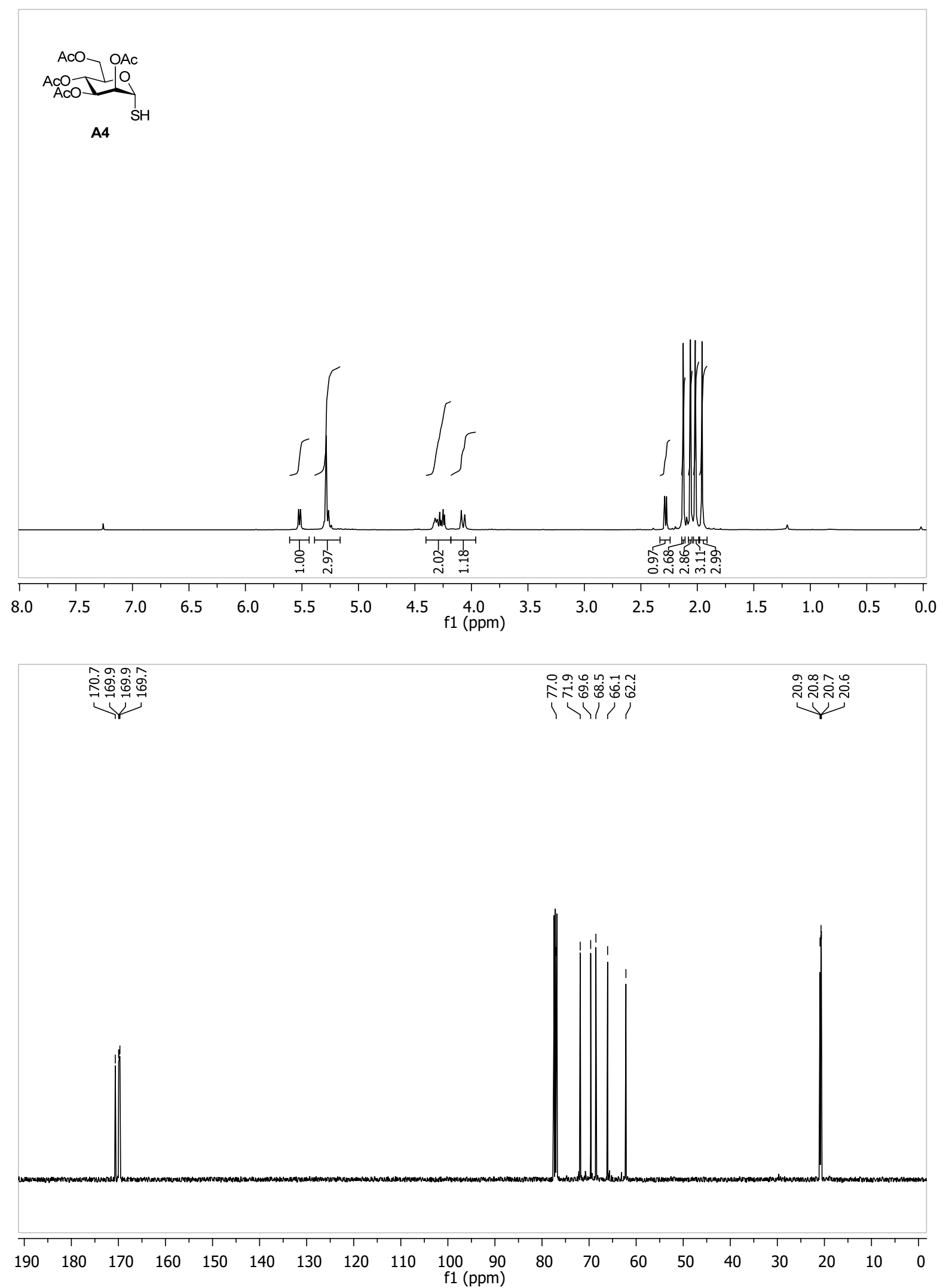

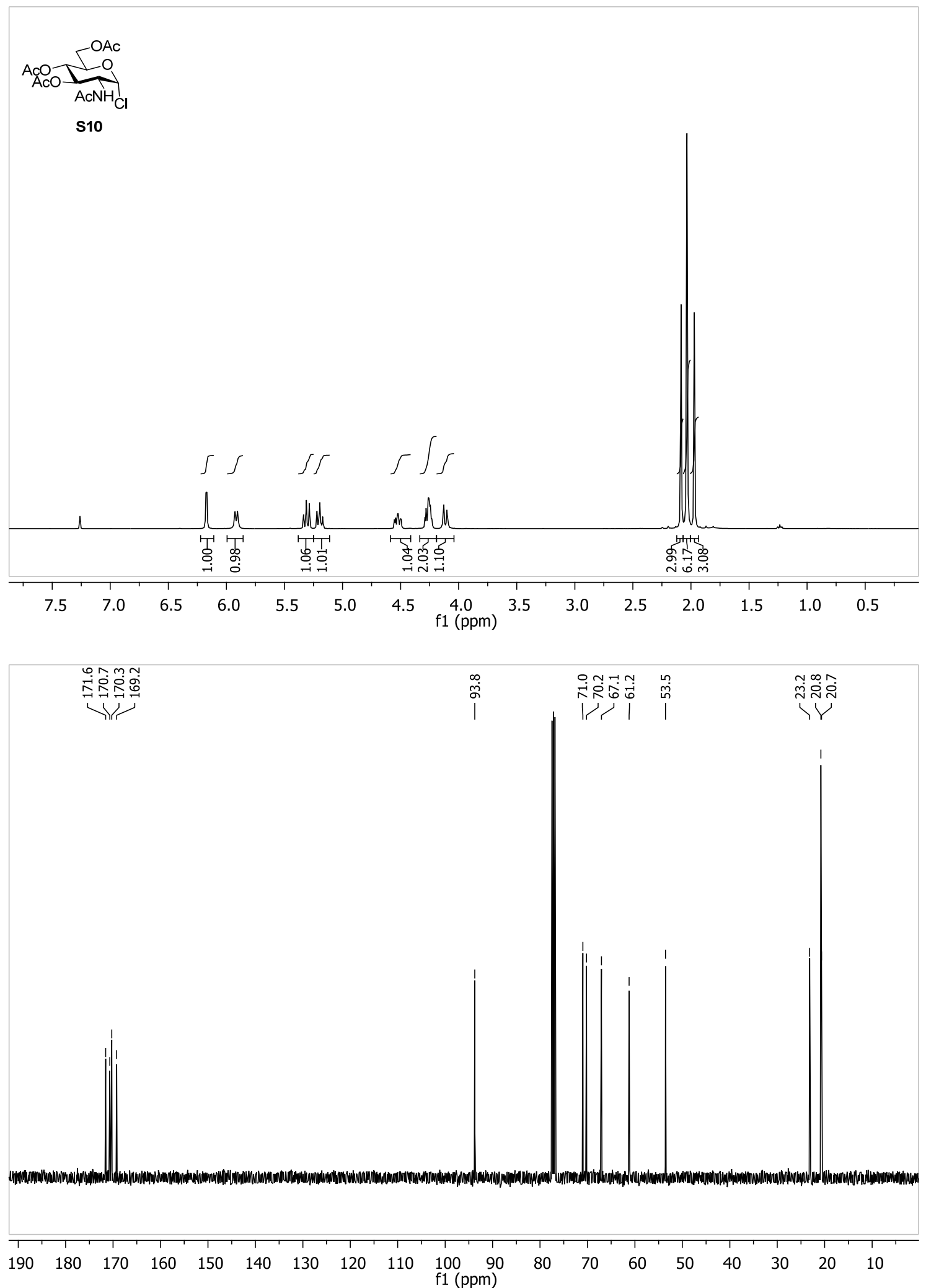

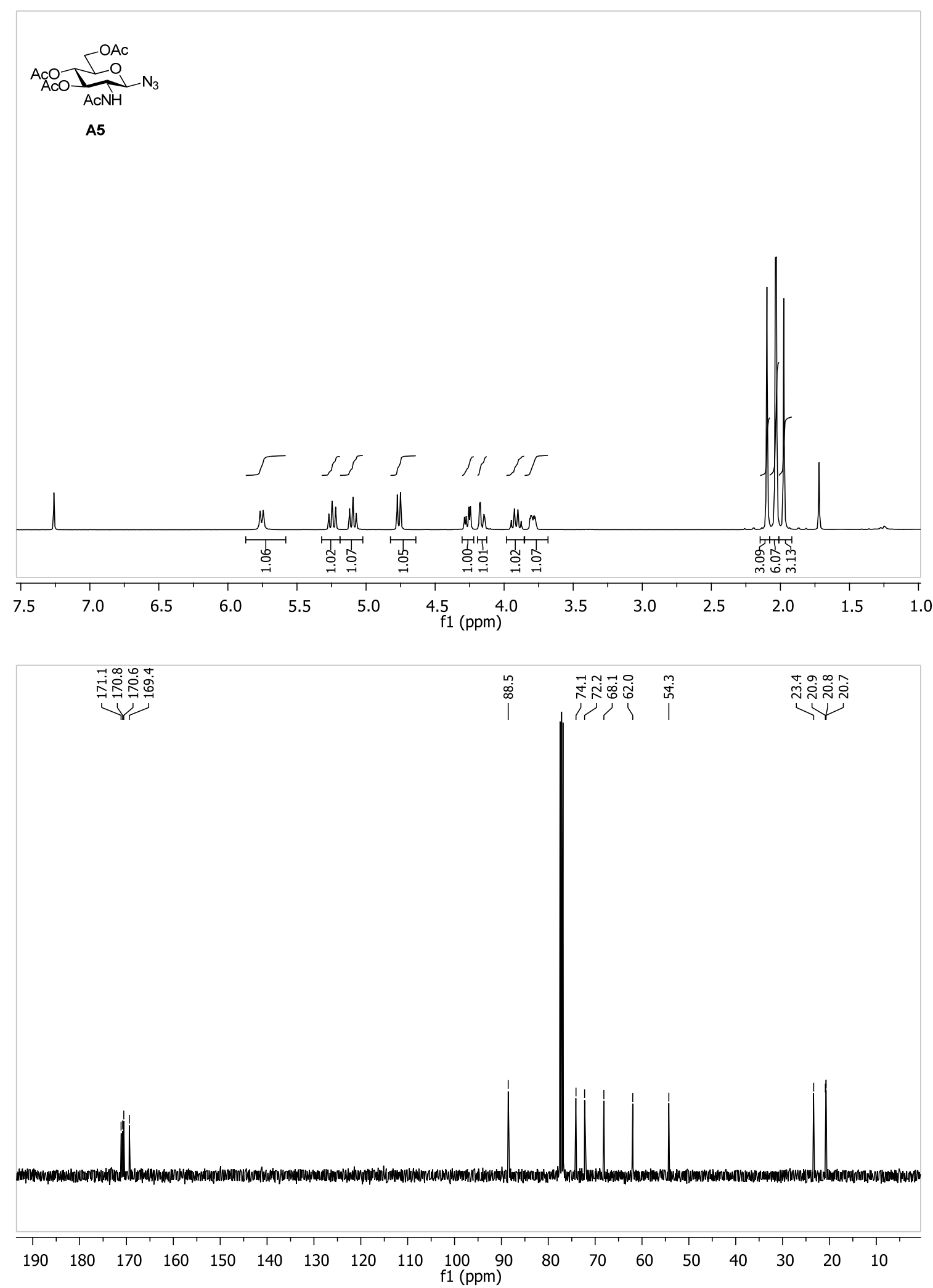

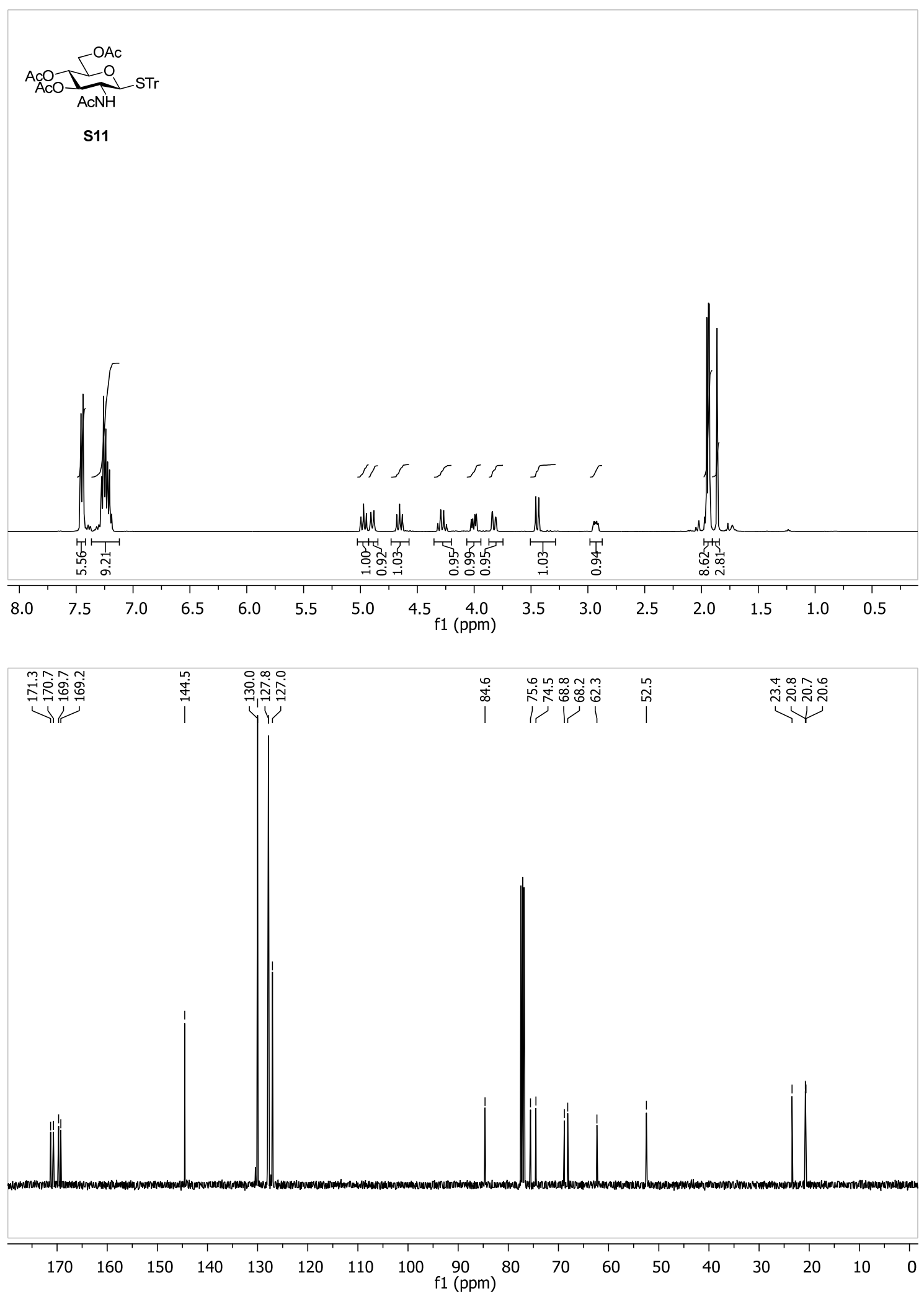

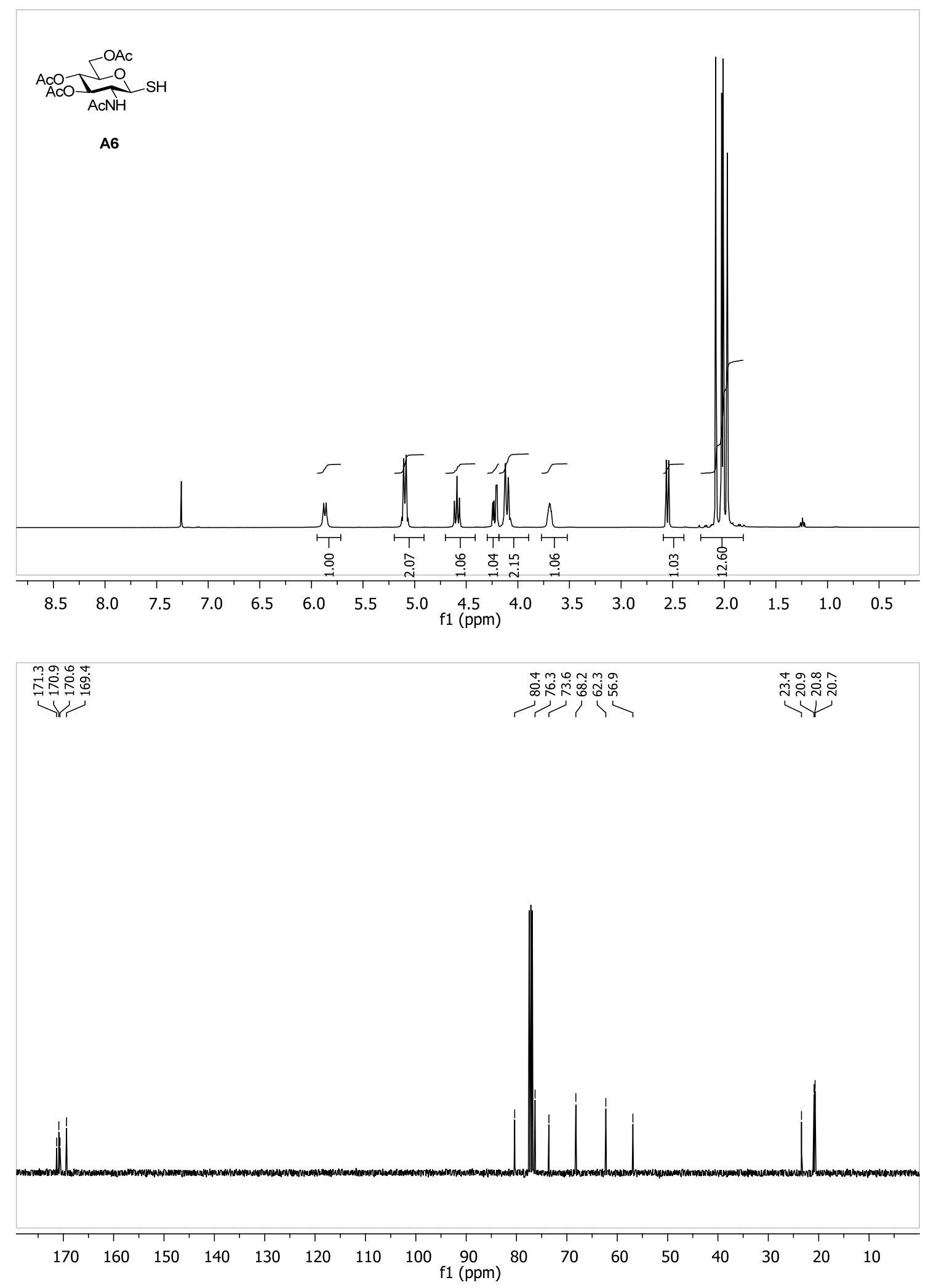


\section{Gropu B building blocks}

$\beta$-D-Galactopyranosyl azide S12
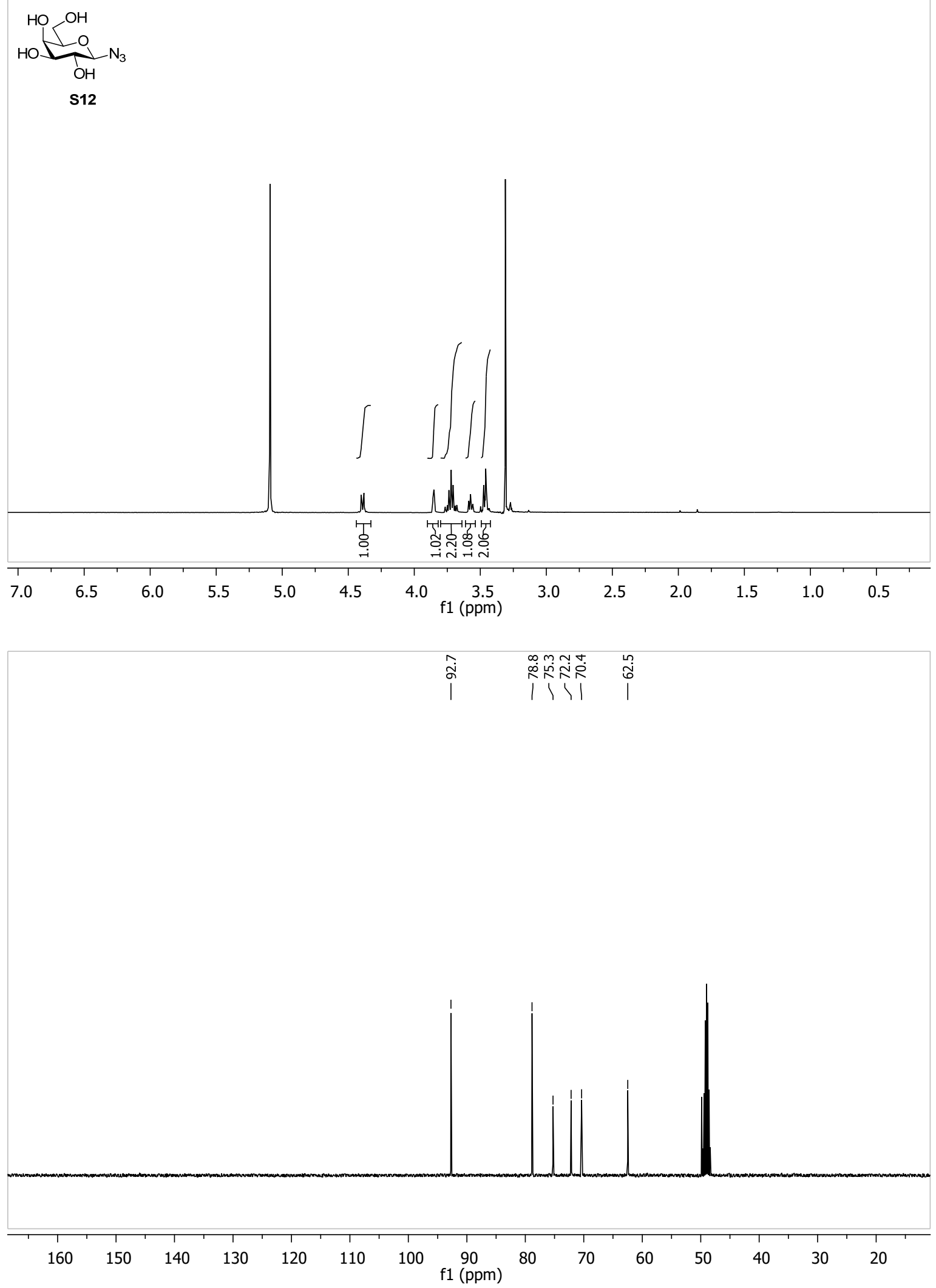


\section{2,4,6-Tri-O-acetyl-3-O-allyl- $\beta$-D-galactopyranosyl azide B1}
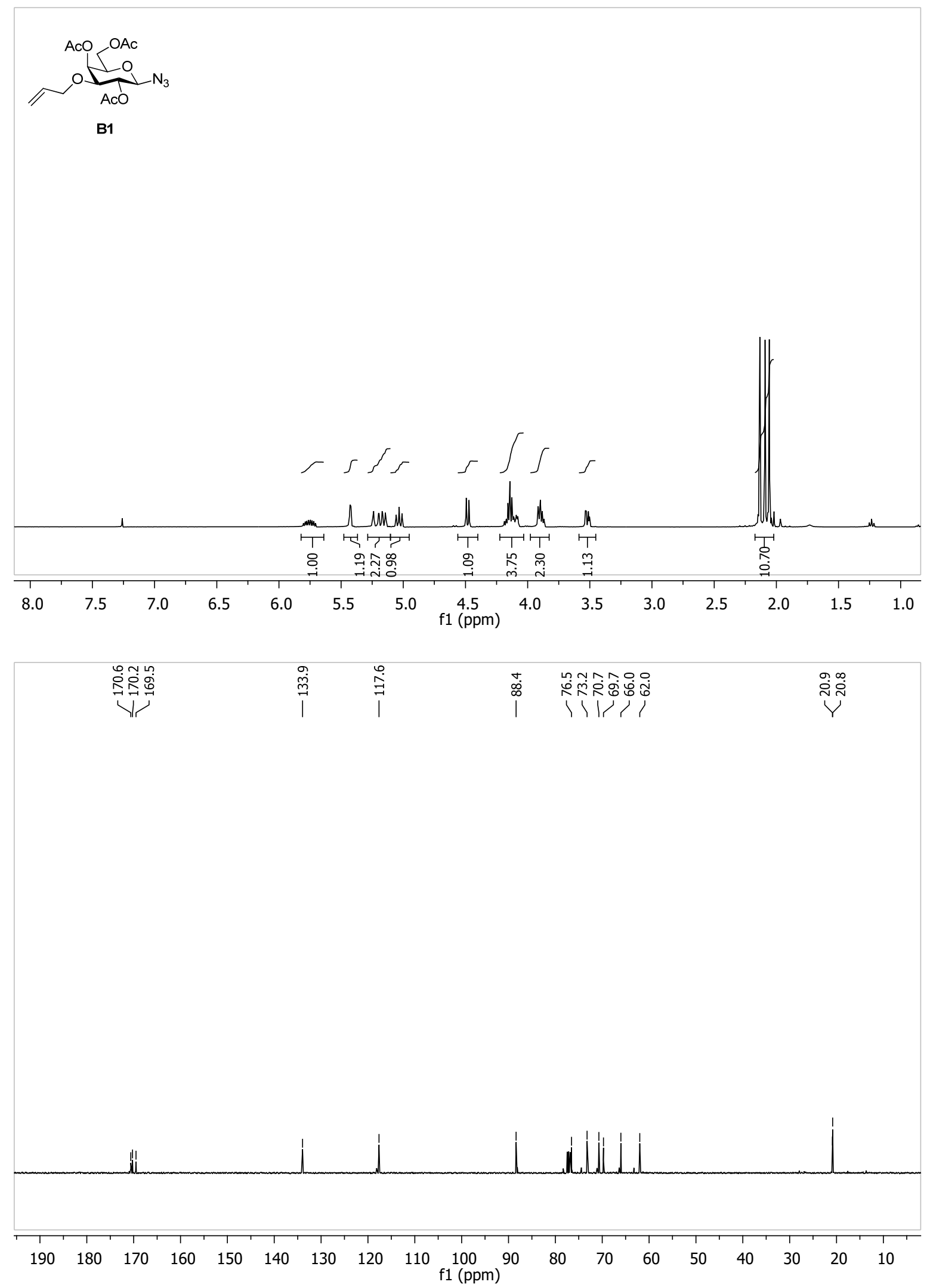

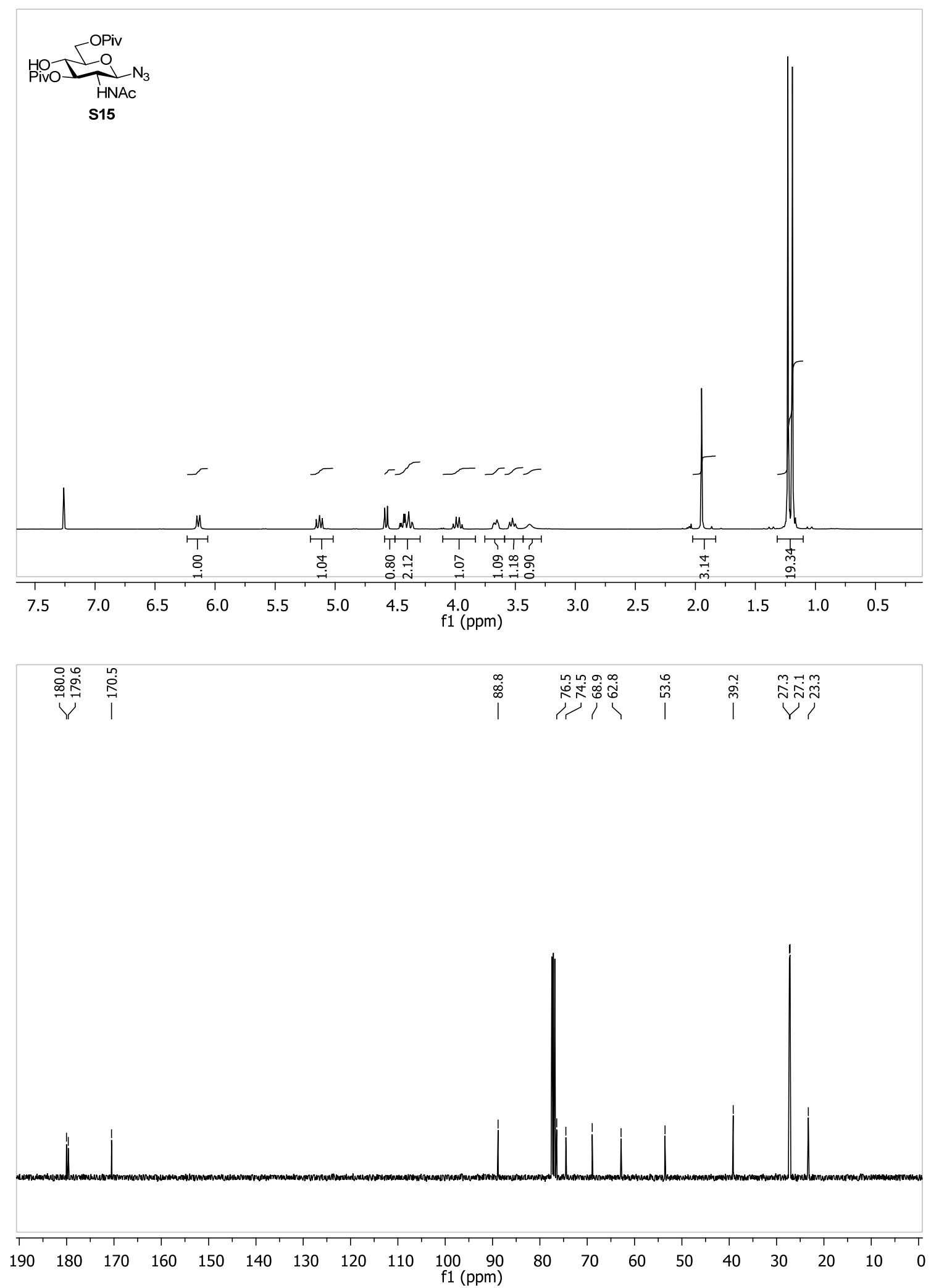


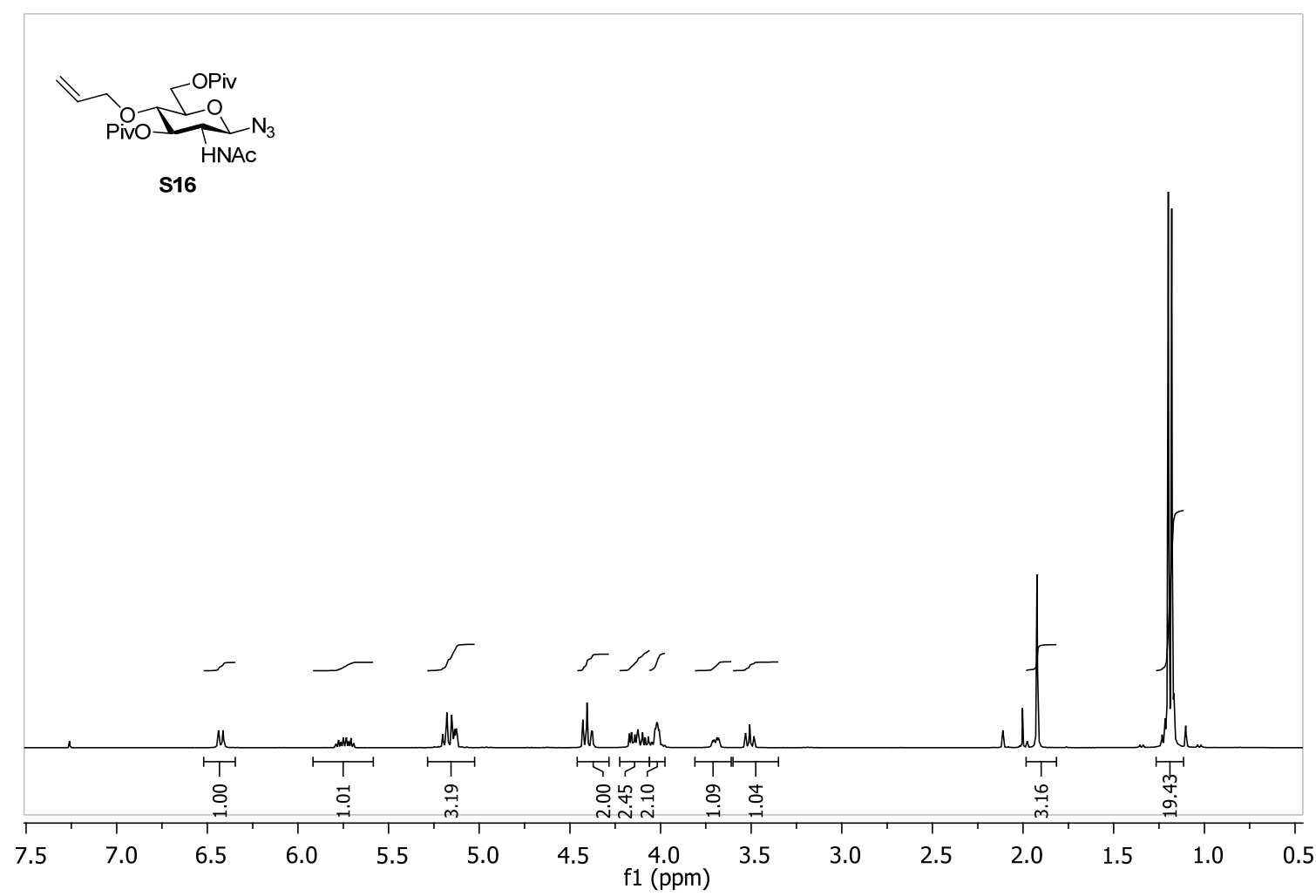

至

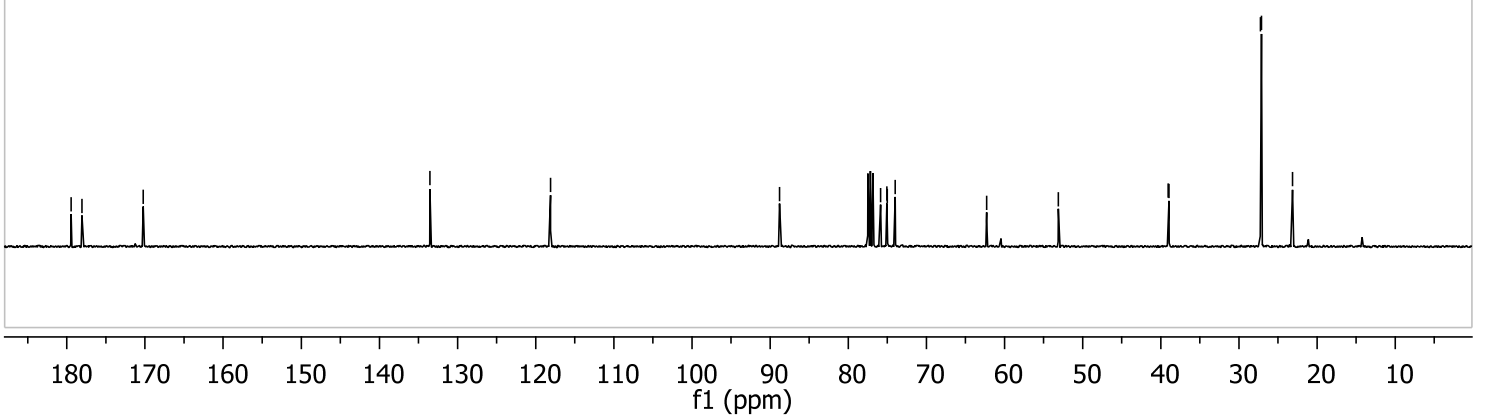



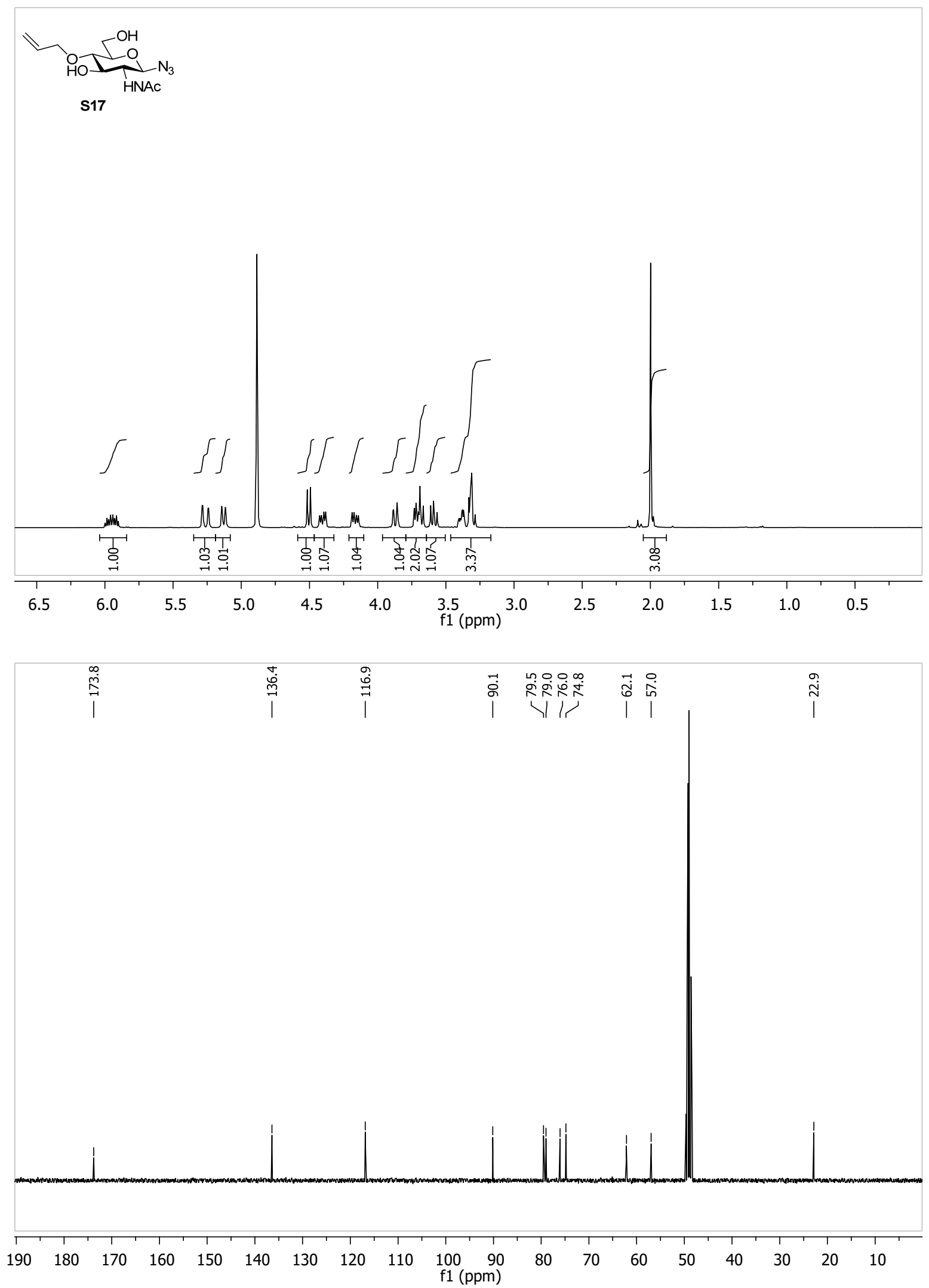

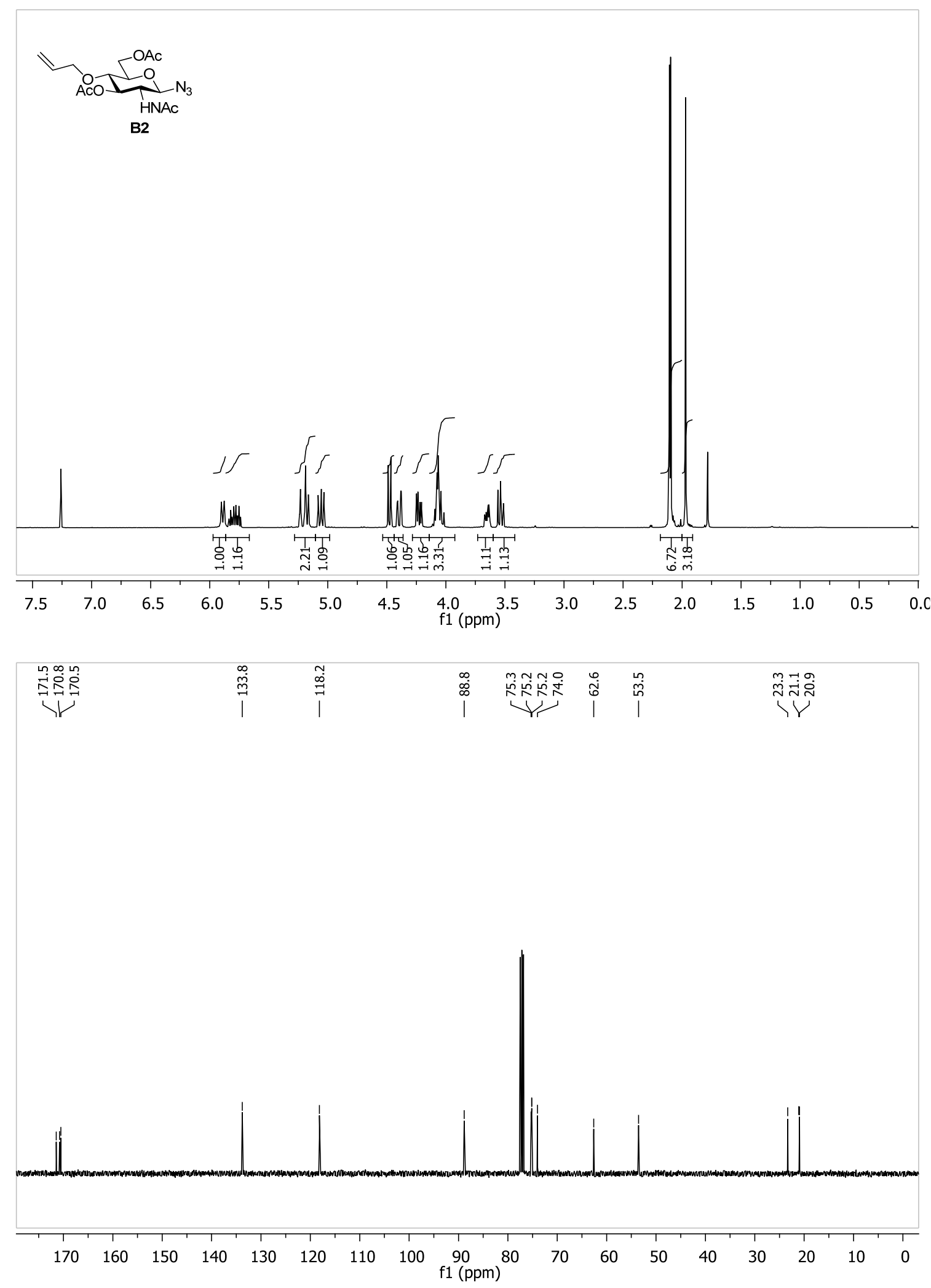

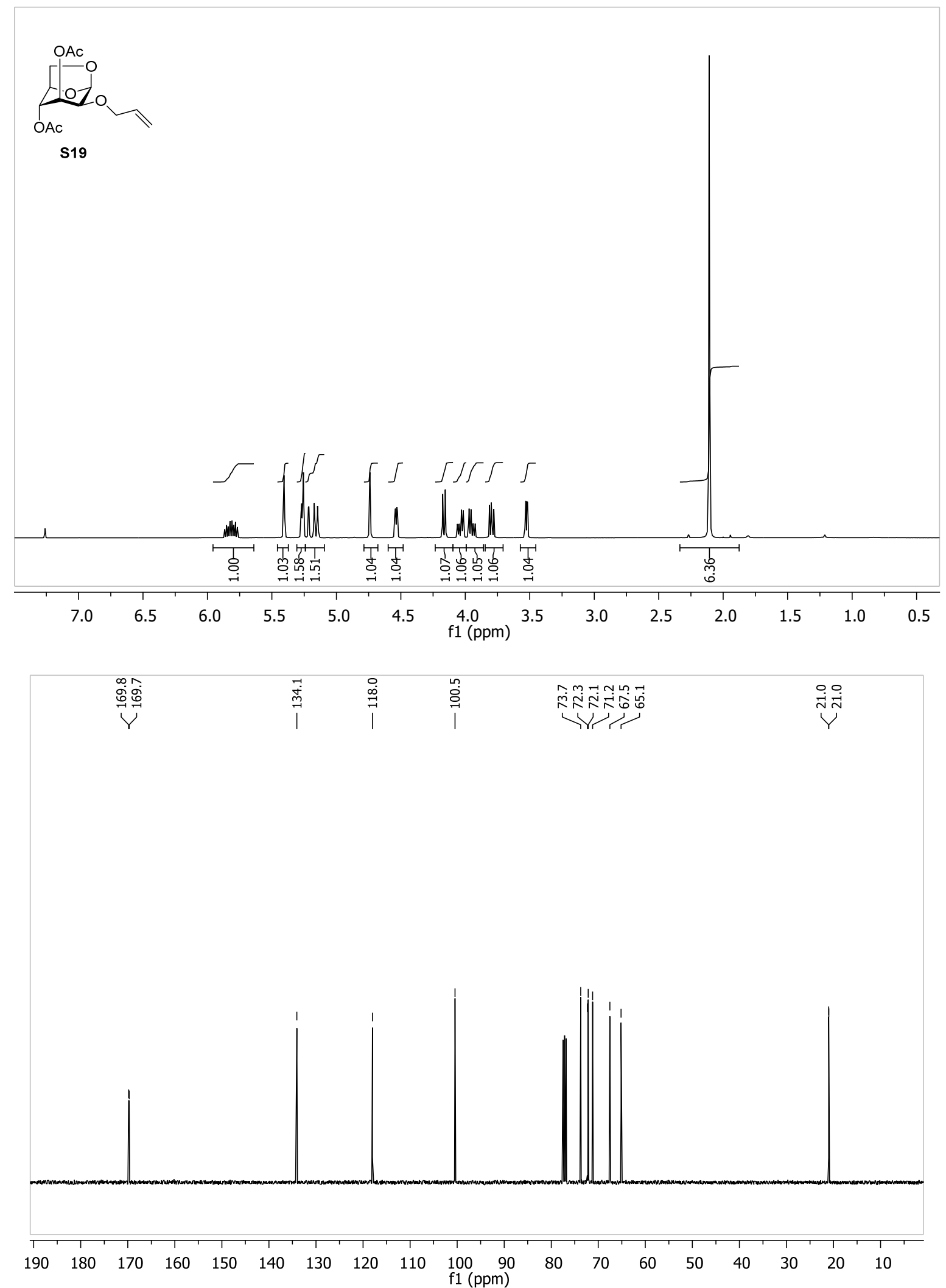

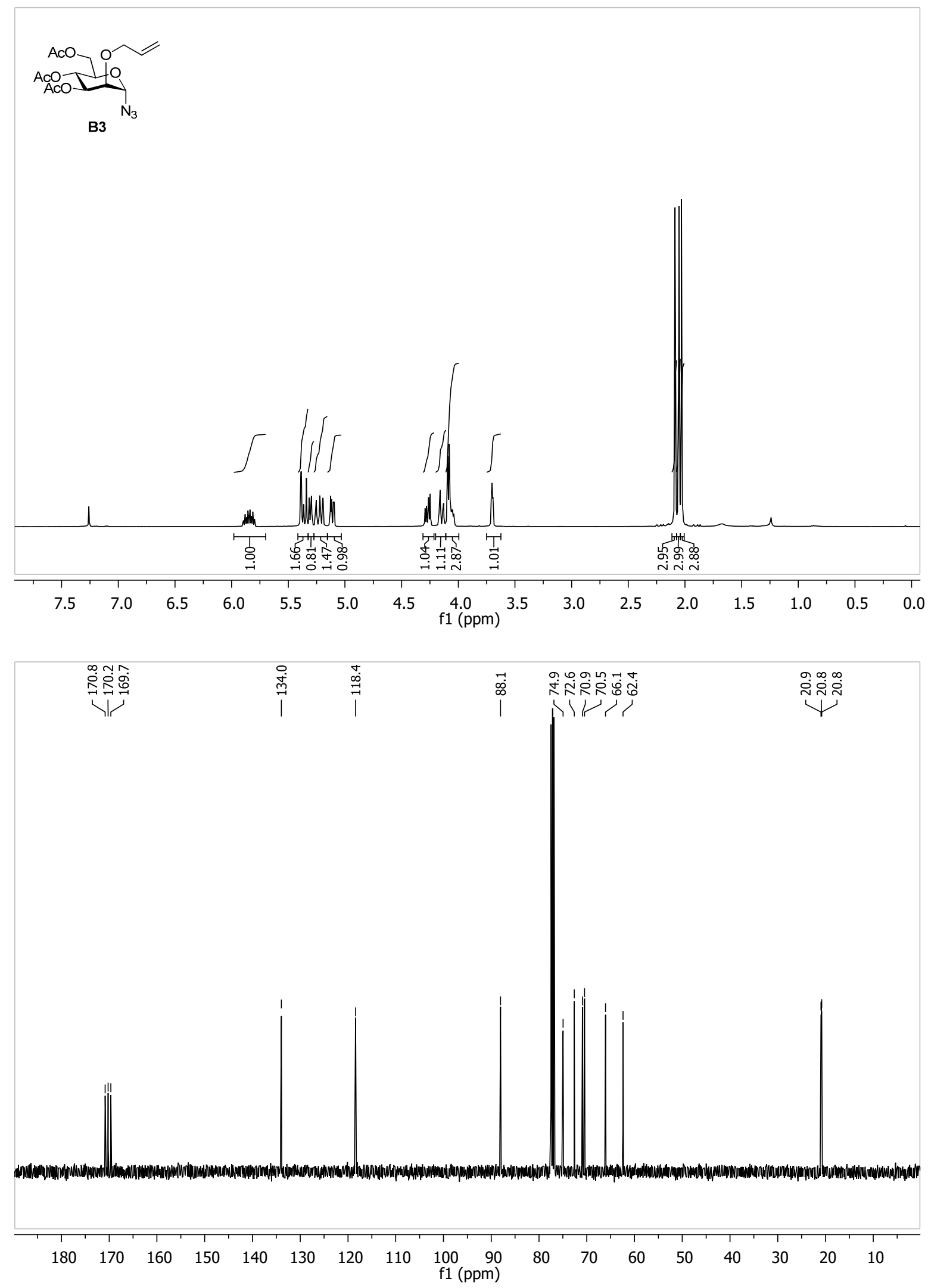


\section{Group C building blocks}

Triphenylmethyl 1-thio-2,3,4,6-tetra-O-acetyl- $\beta$-D-galactopyranoside S20
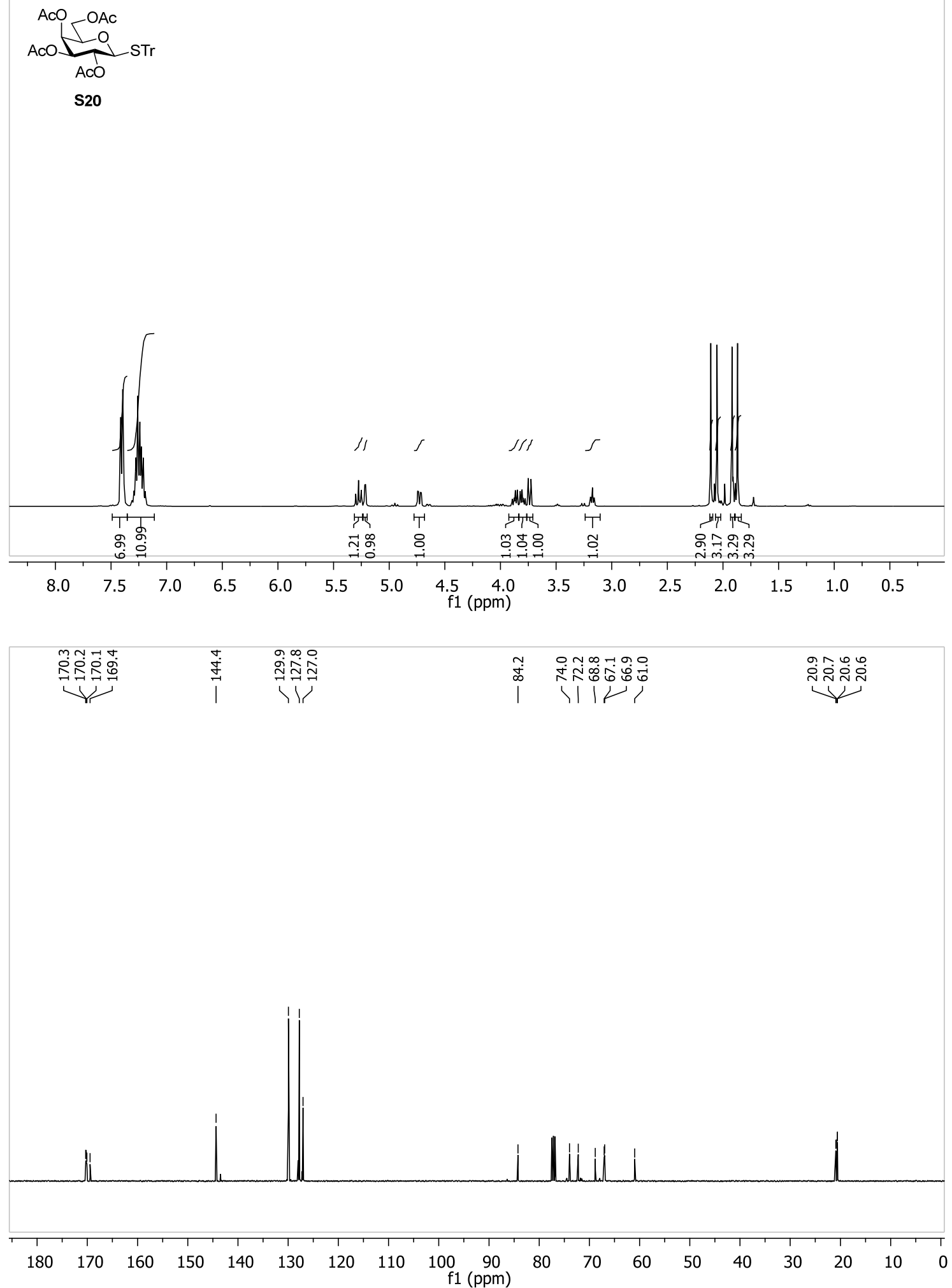

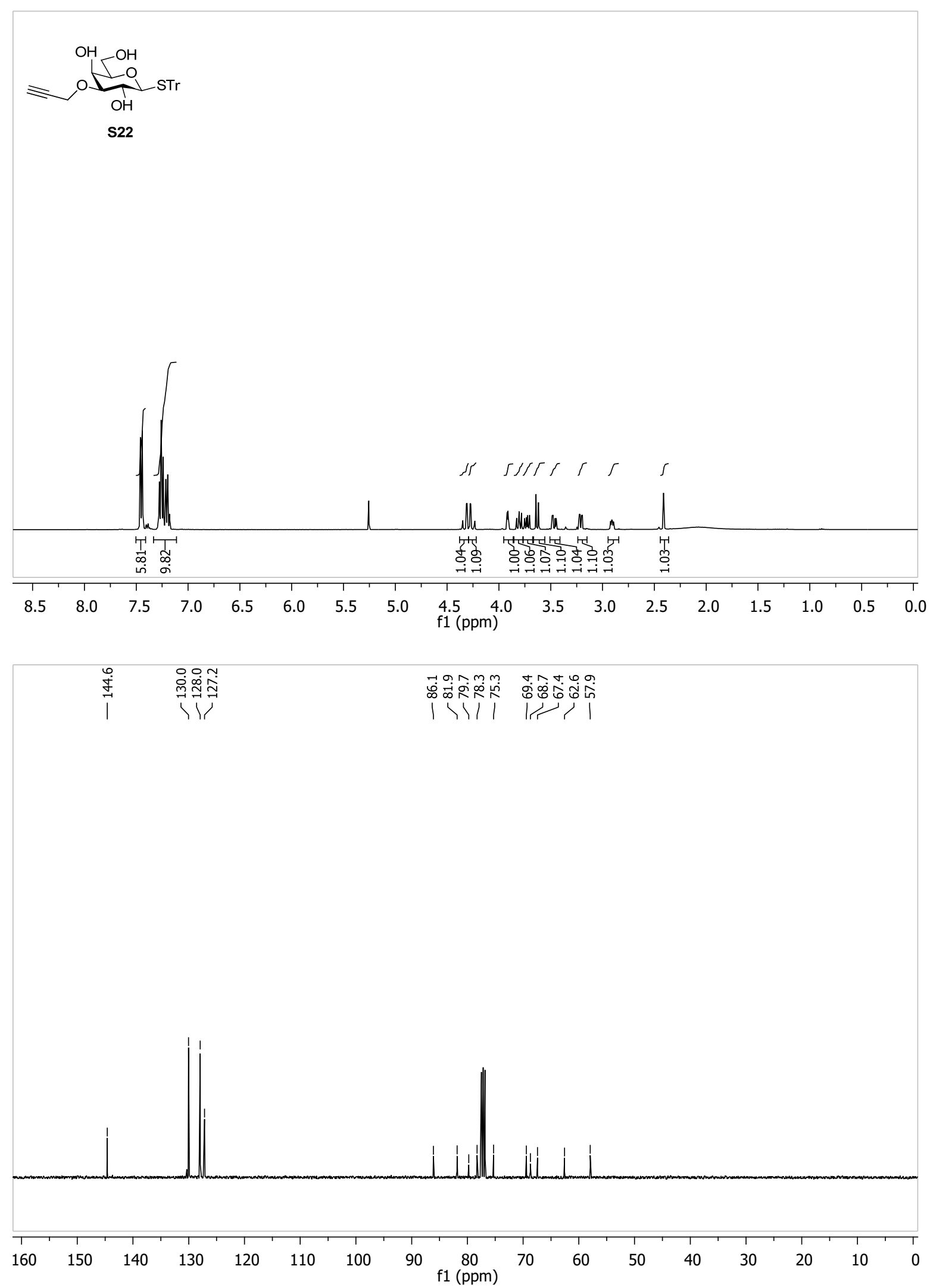
Triphenylmethyl 1-thio-2,4,6-tri-O-acetyl-3-propargyl- $\beta$-D-galactopyranoside S23
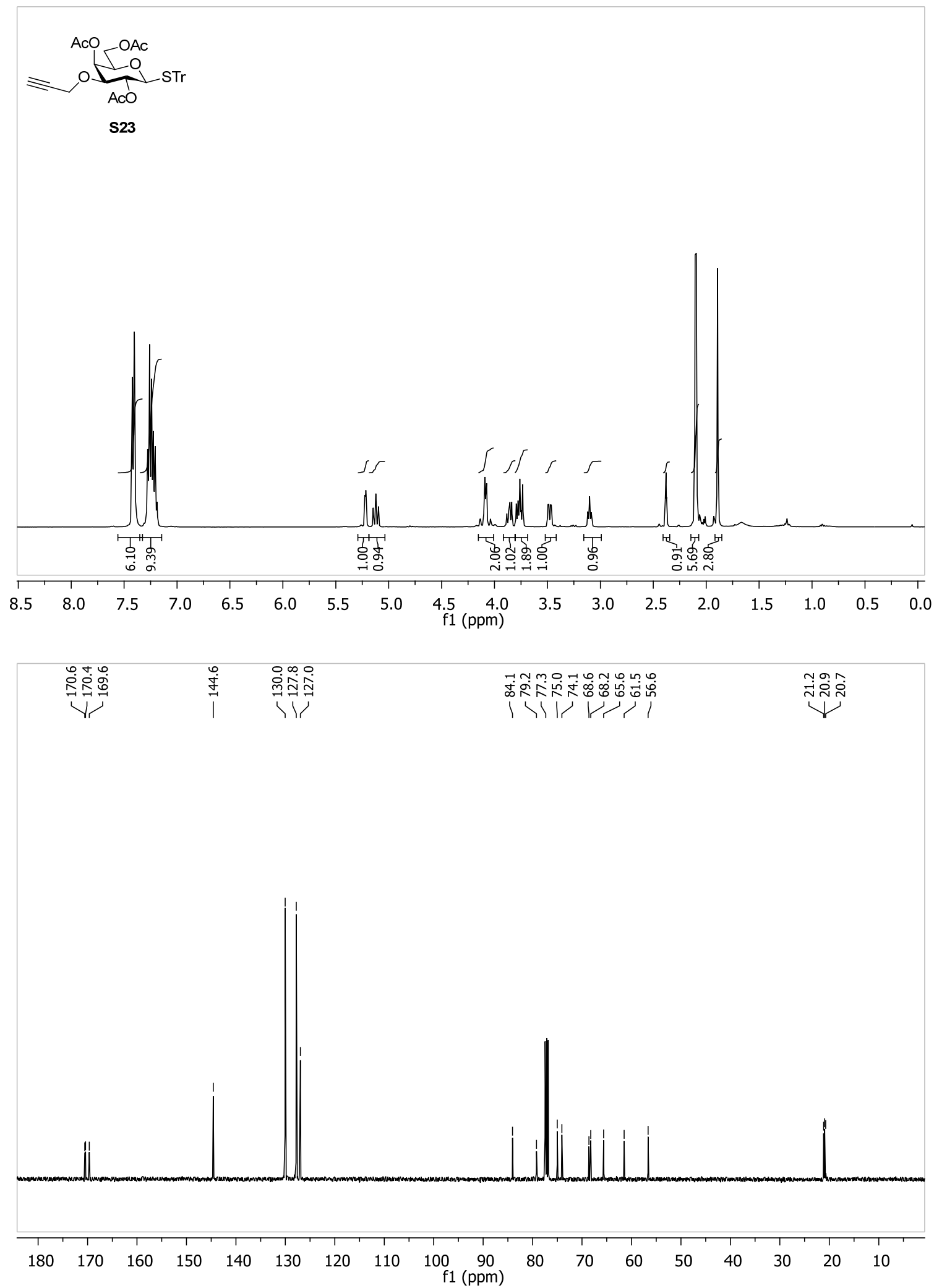
2,4,6-Tri-O-acetyl-3-propargyl- $\beta$-D-galactopyranosyl thiol S24
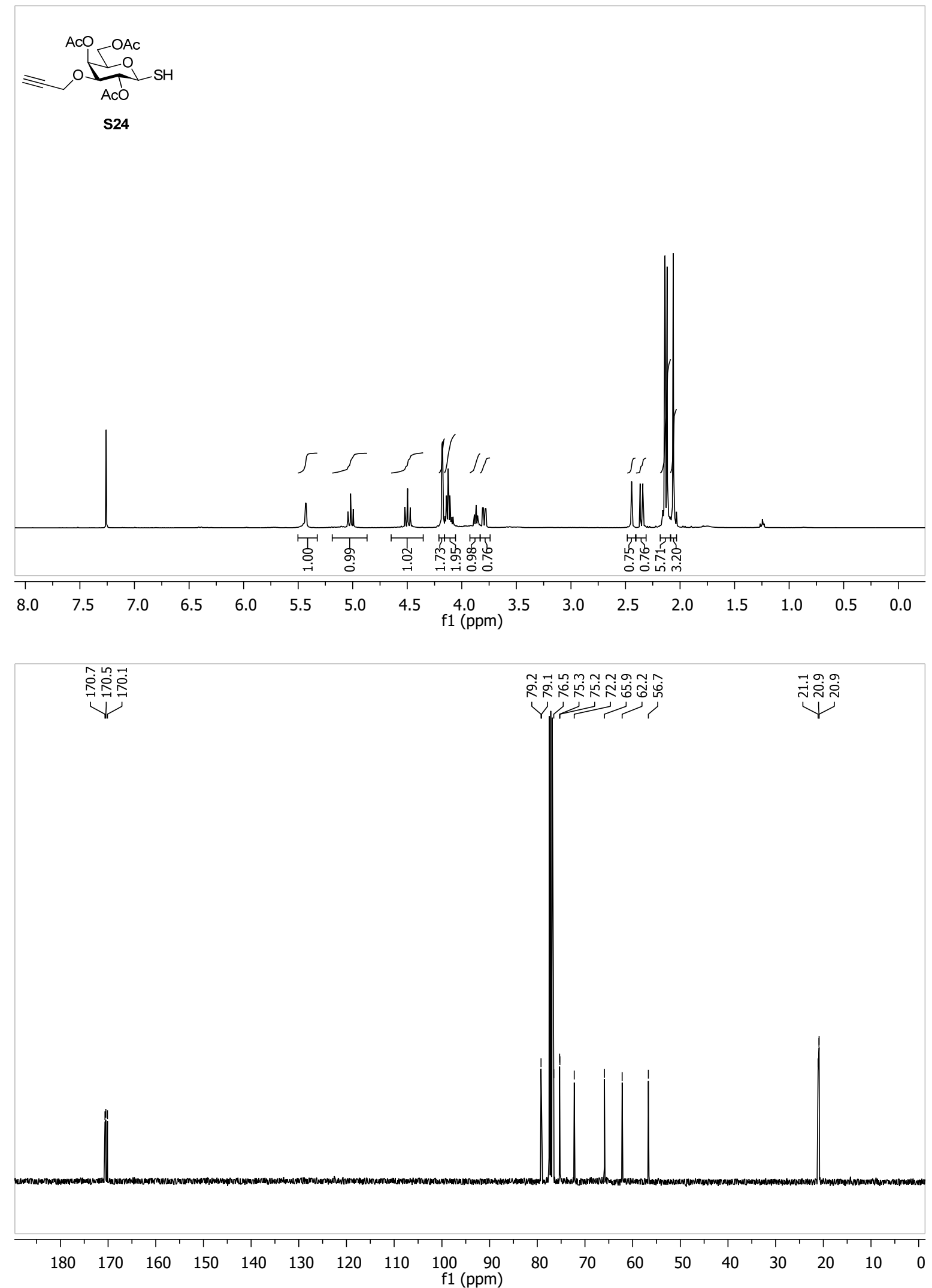
1-S-acetyl-2,4,6-tri-O-acetyl-3-propargyl-1-thio- $\beta$-D-galactopyranoside C1
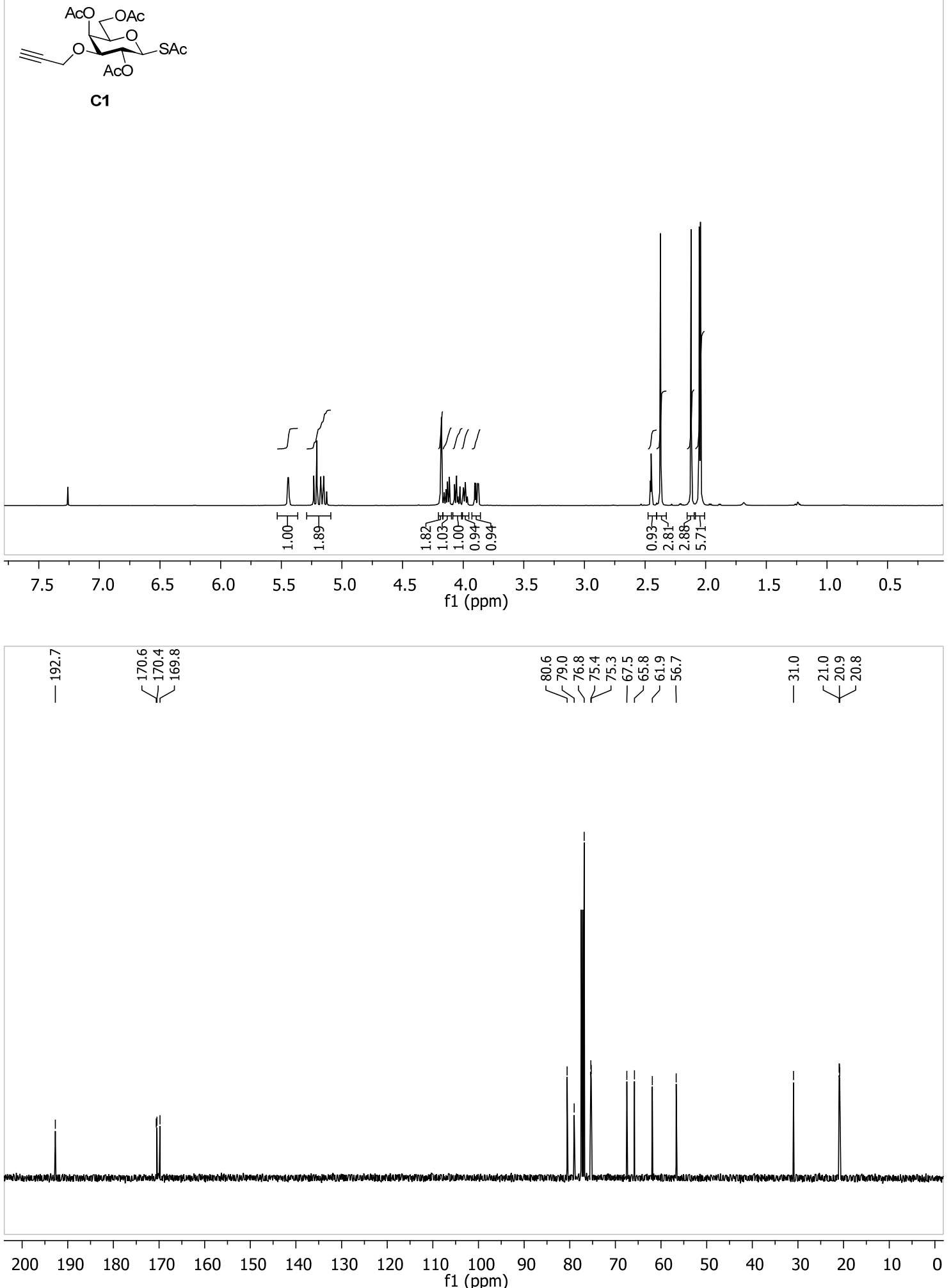
Triphenylmethyl 1-thio-2-acetamido-2-deoxy-3,6-tri-O-pivaloyl- $\beta$-D-glucopyranoside S26
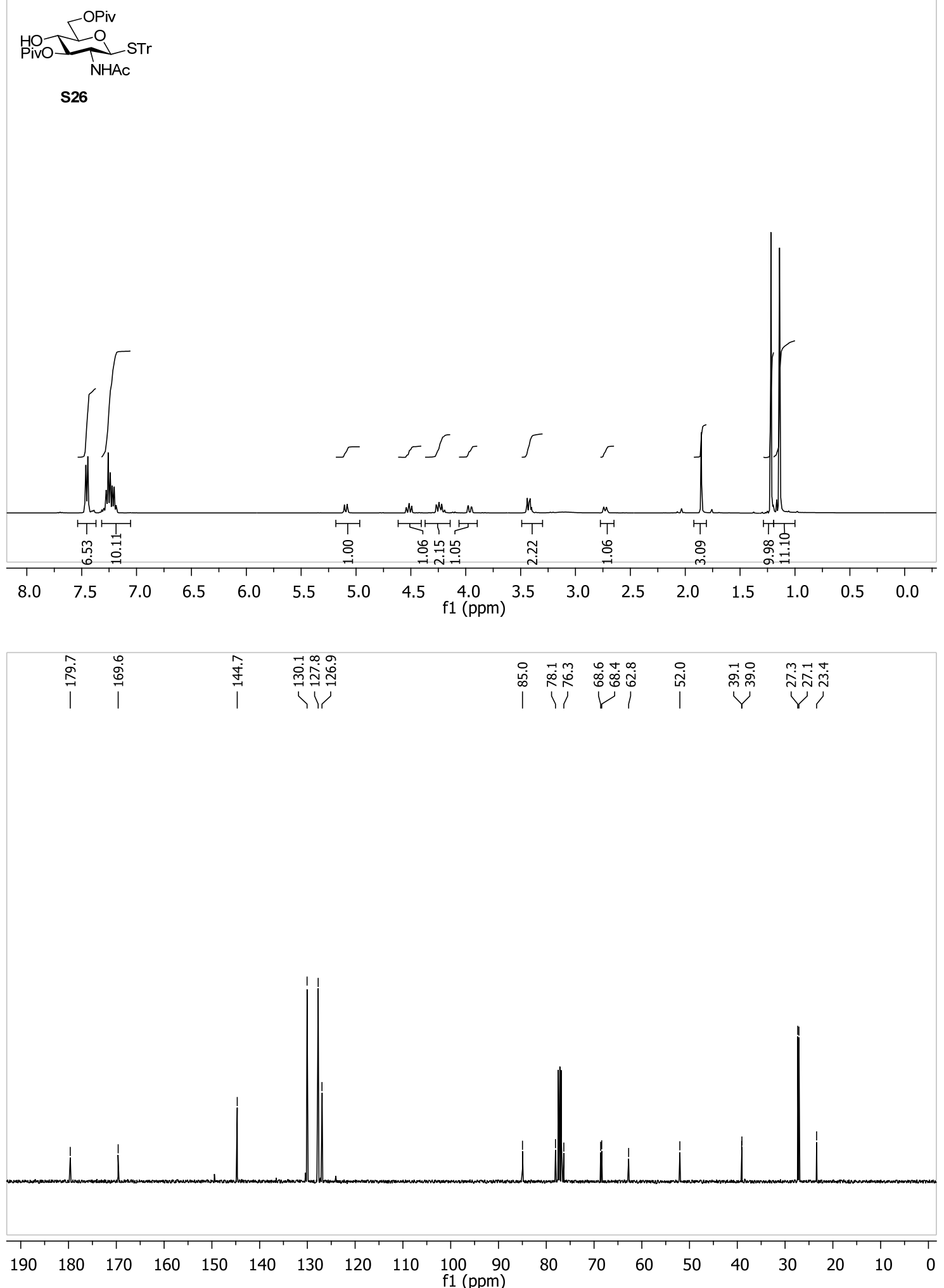
Triphenylmethyl 1-thio-2-acetamido-2-deoxy-3,6-tri-O-pivaloyl-4-O-propargyl- $\beta$-D-glucopyranoside S27
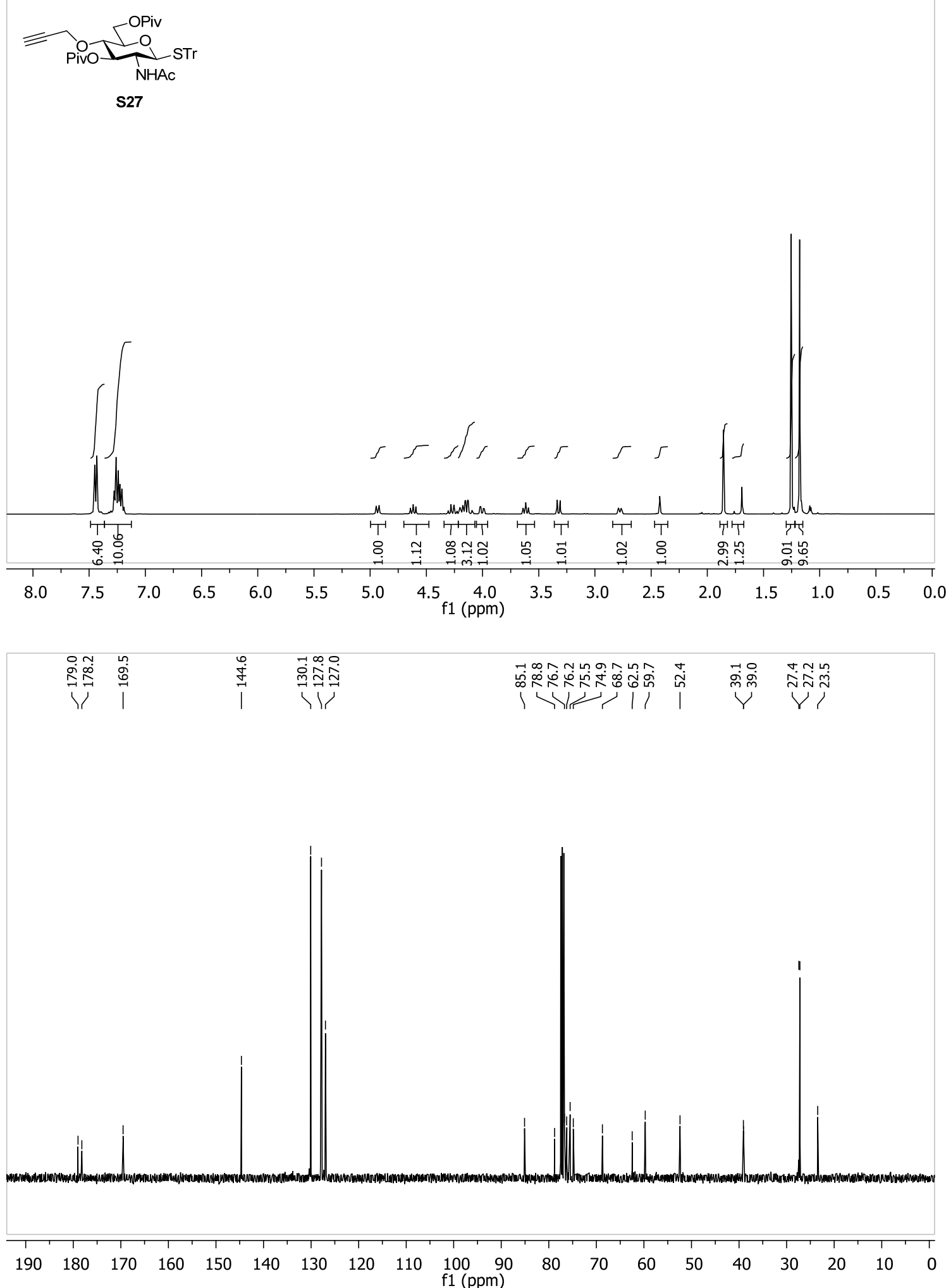
2-Acetamido-1-S-acetyl-2-deoxy-3,6-di-O-acetyl-4-O-propargyl- $\beta$-D-1-thio-glucopyranose C2
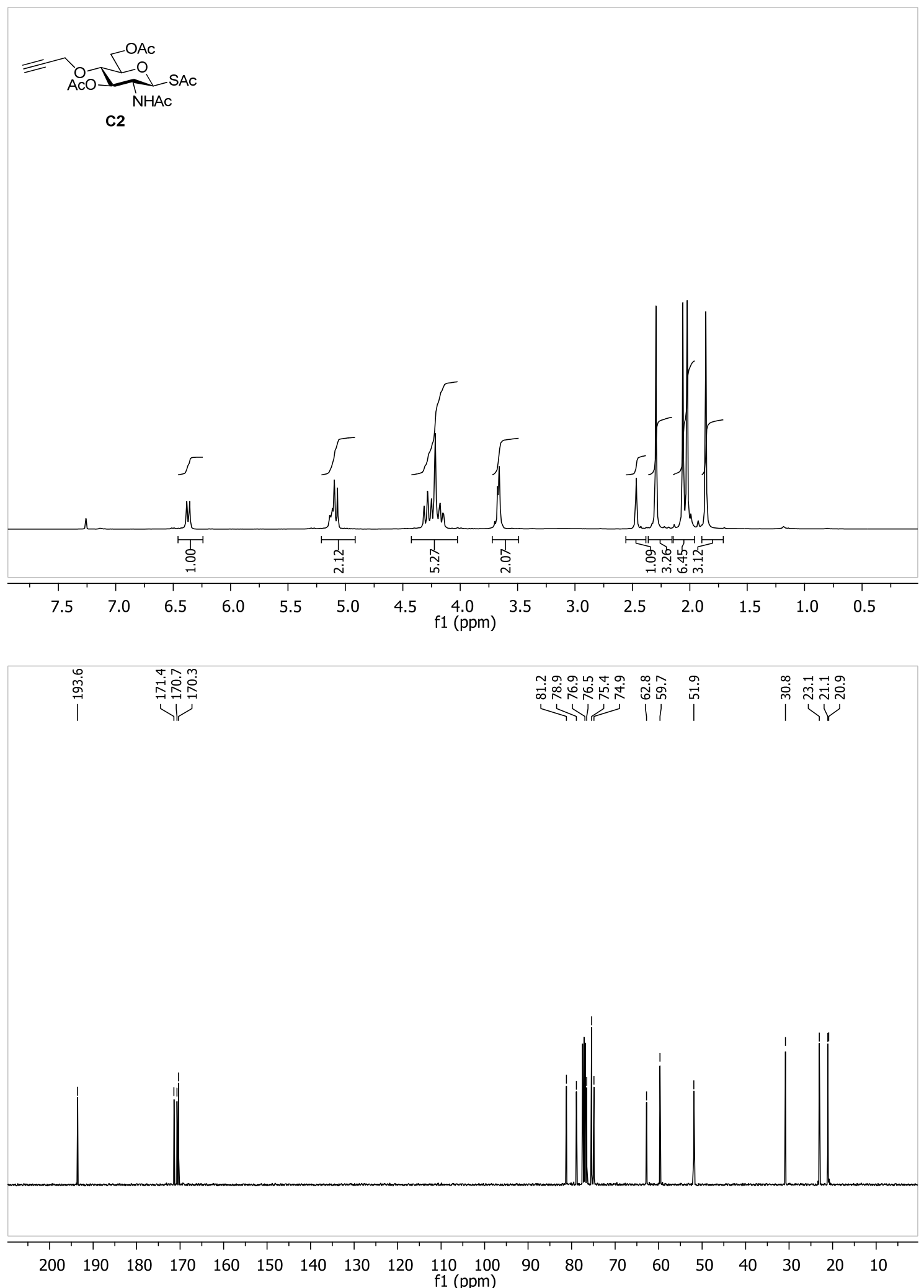
1,6-anhydro-3,4-O-acetyl-2-O-propargyl- $\beta$-D-mannopyranose S29
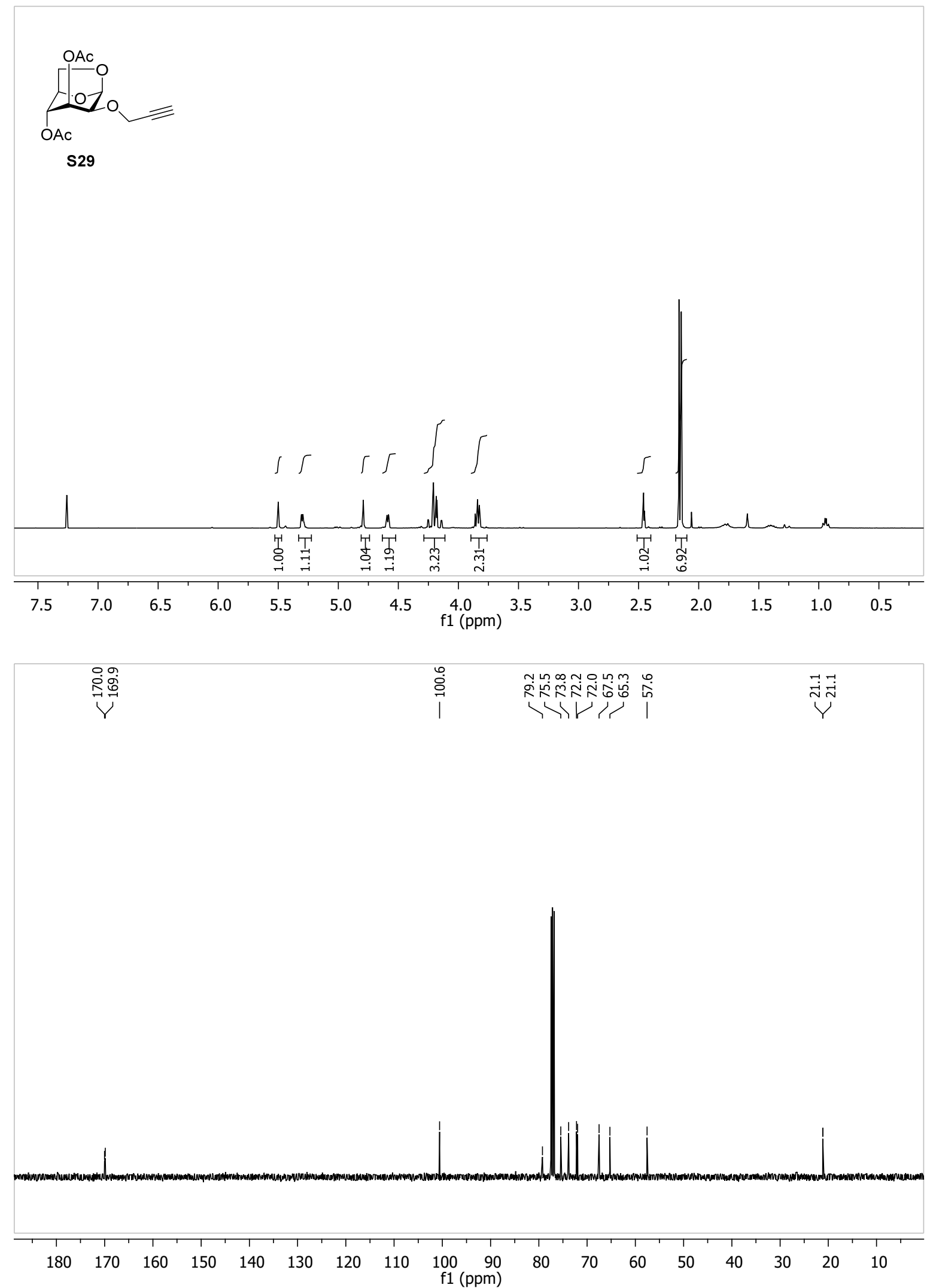


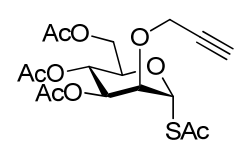

C3
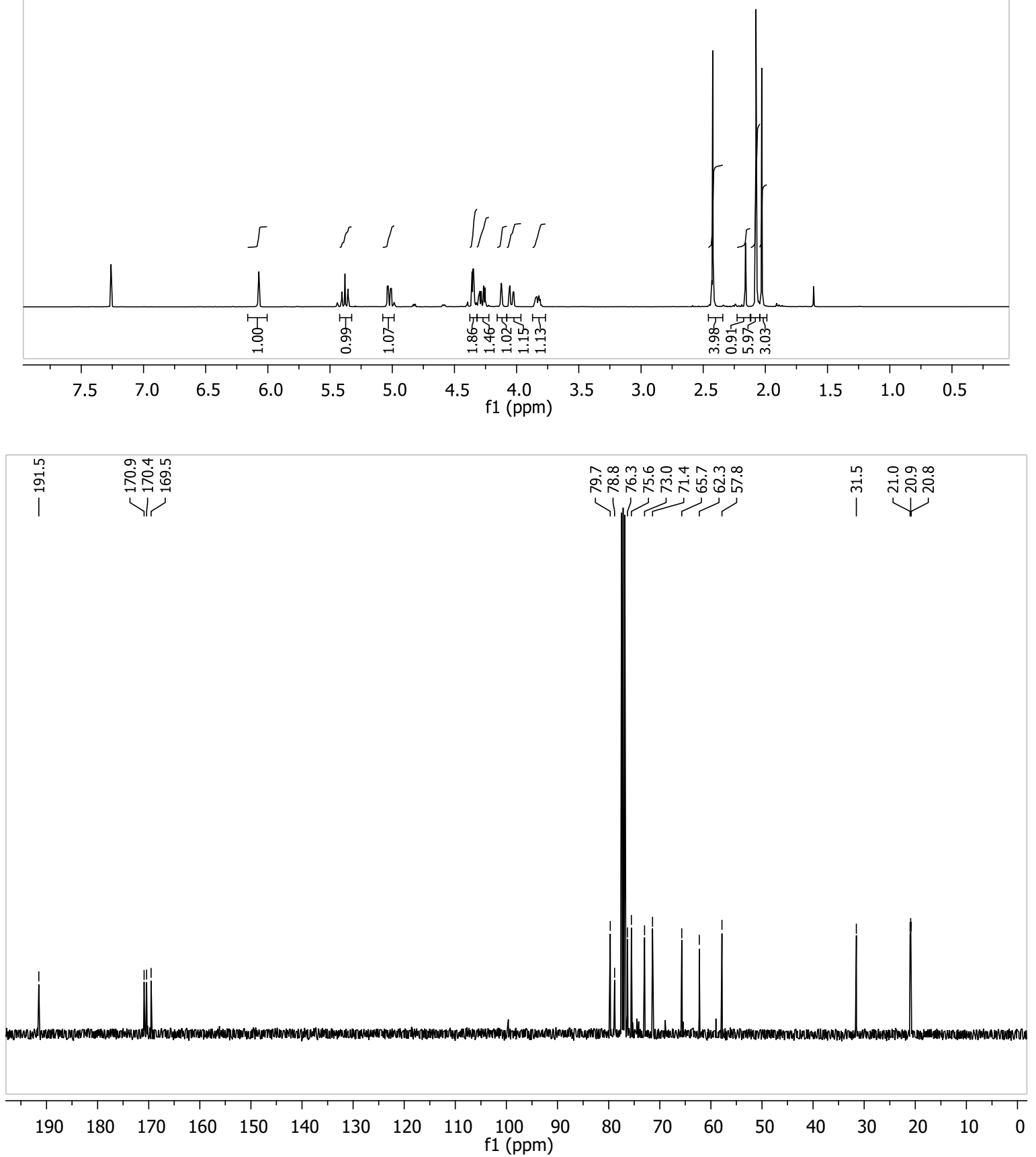
Group C building blocks

tButyl allylcarbamate
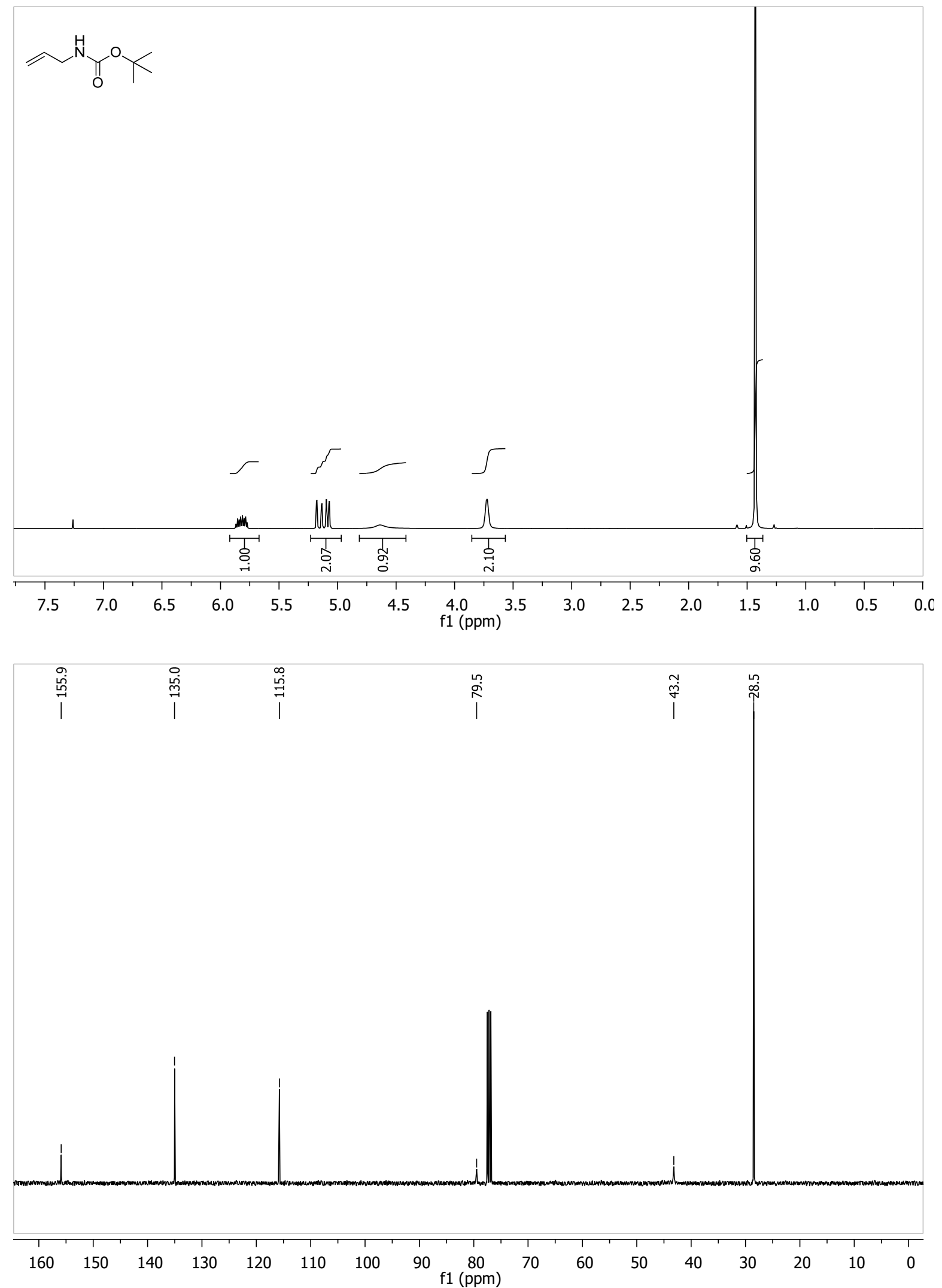
1-(tButyl(3-mercaptopropyl)carbamate) 2,3,4,6-tetra-O-acetyl- $\beta$-D-galactopyranoside S30
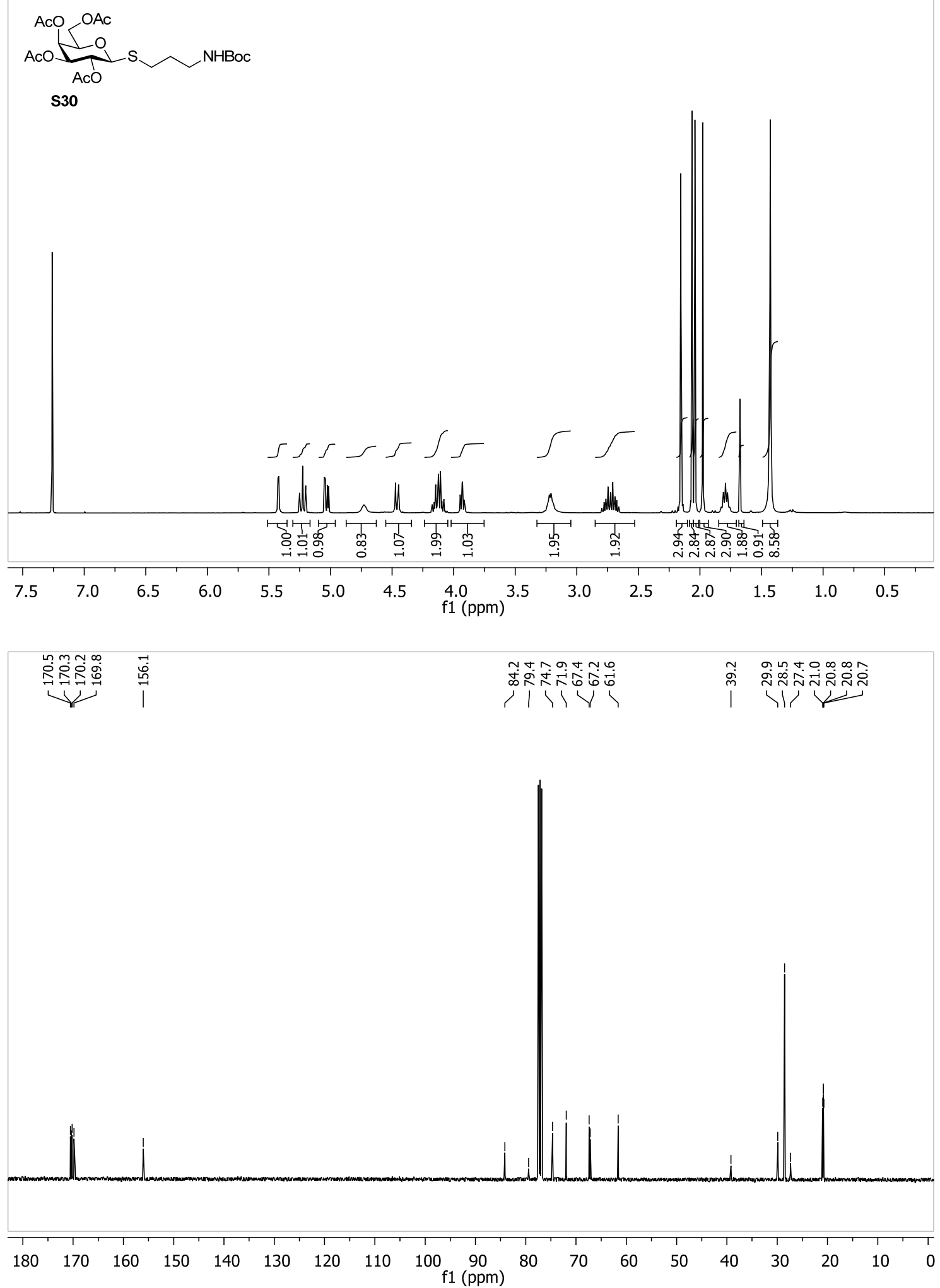
1-(tButyl(3-mercaptopropyl)carbamate) 2,4,6-tri-O-acetyl-3-O-propargyl- $\beta$-D-galactopyranoside D1
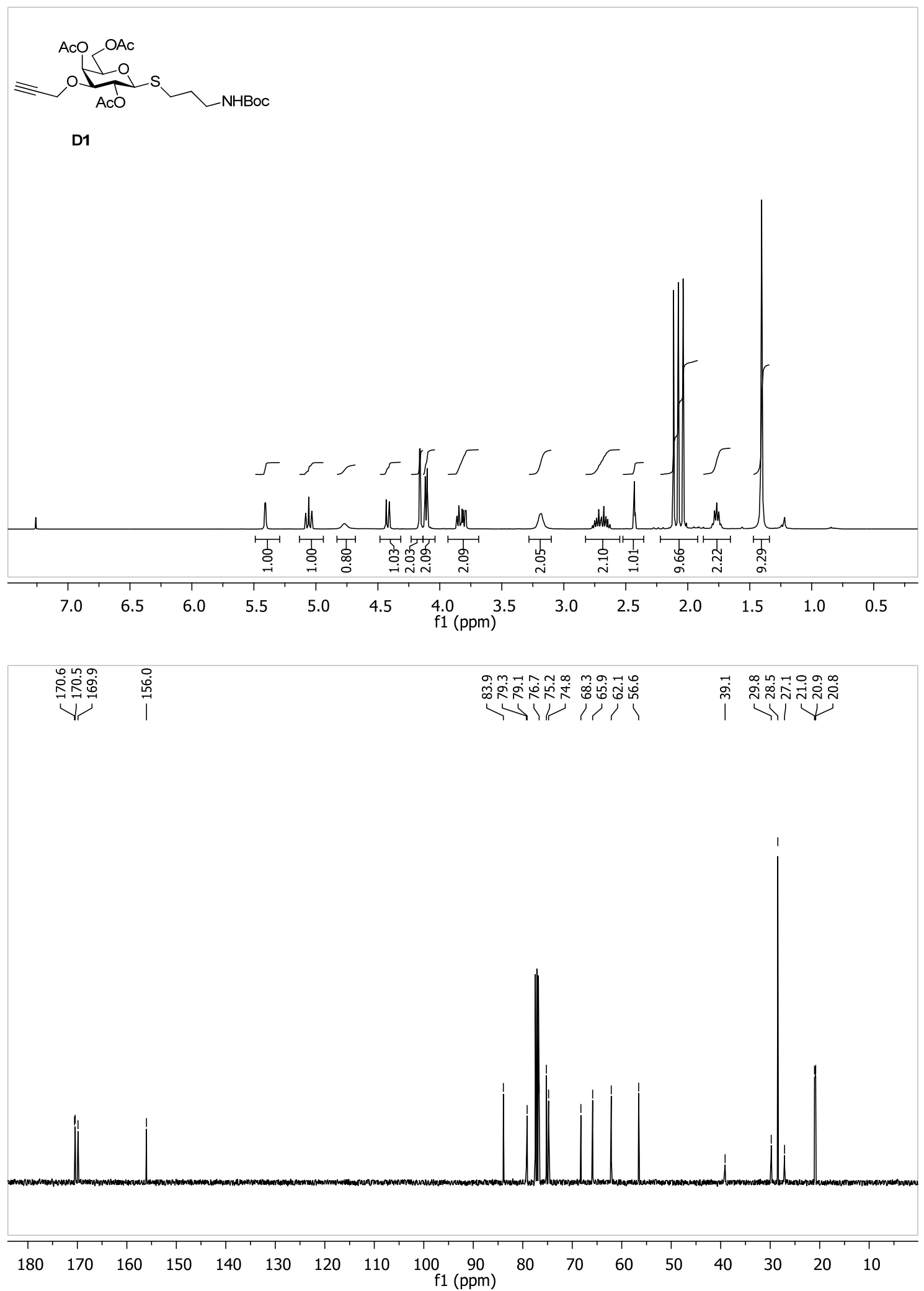
1-(tButyl(3-mercaptopropyl)carbamate) 2,4,6-tri-O-acetyl-3-O-allyl- $\beta$-D-galactopyranoside D2
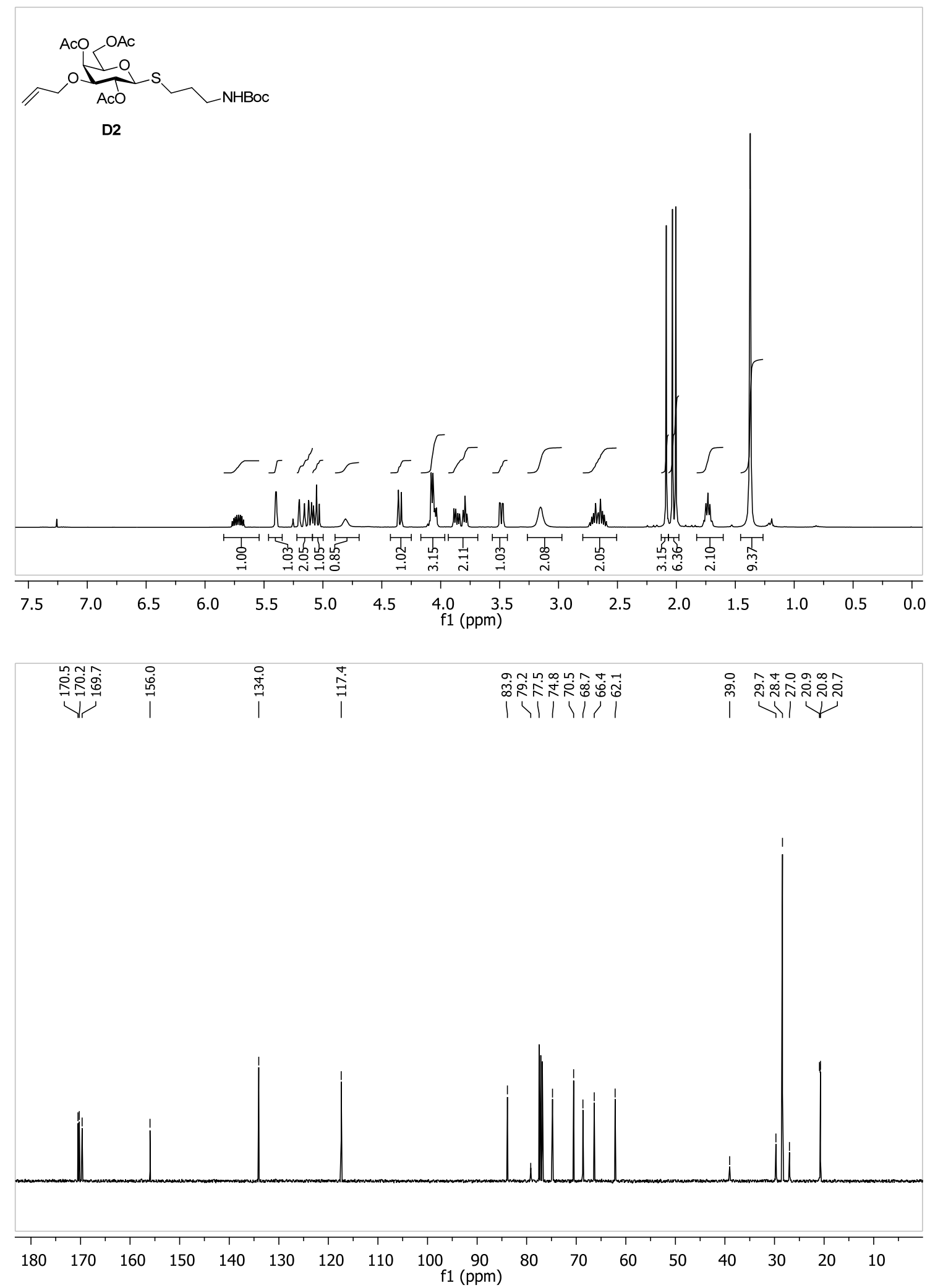


\section{Protected glycan mimics}

1-(tButyl(3-mercaptopropyl)carbamate)

2,4,6-tri-O-acetyl-3-O-[(2',4',6'-tri-O-acetyl-3'-O-[(2",3",4",6"-tetra-Oacetyl-1"-thio- $\beta$-D-galactopyranosyl)propyl]-- $\beta$-D-galactopyranosyl)-1H-[1,2,3]-triazol-4-yl-methyl]- $\beta$-Dgalactopyranoside S34
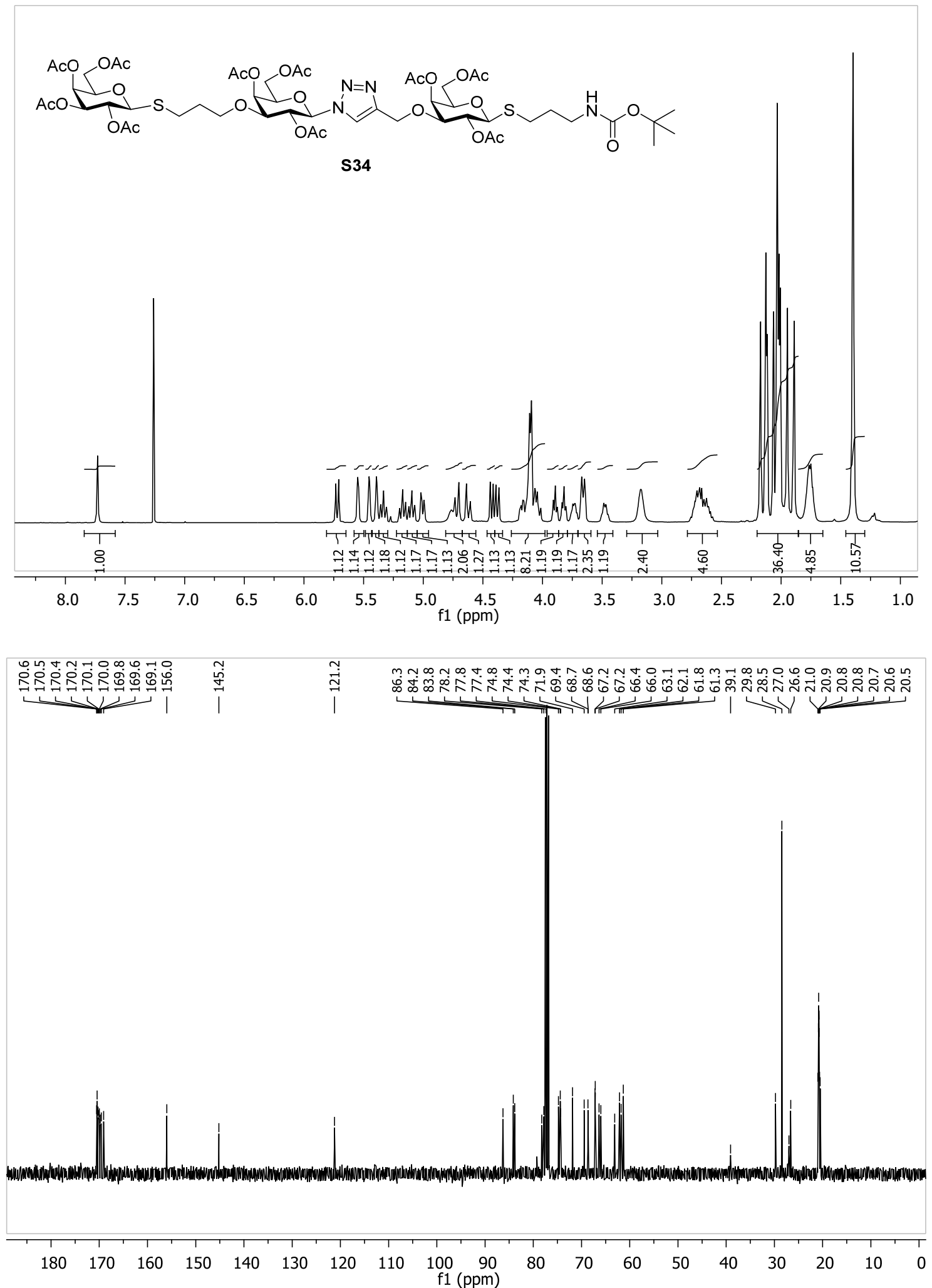
1-(tButyl(3-mercaptopropyl)carbamate)

2,4,6-tri-O-acetyl-3-O-[(2',4',6'-tri-O-acetyl-3'-O-[(2",3",4",,6"-tetra-Oacetyl- $\beta$-D-galactopyranosyl)-1H-[1,2,3]-triazol-4-yl-methyl]-1'-thio- $\beta$-D-galactopyranosyl)propyl]- $\beta$-Dgalactopyranoside S35
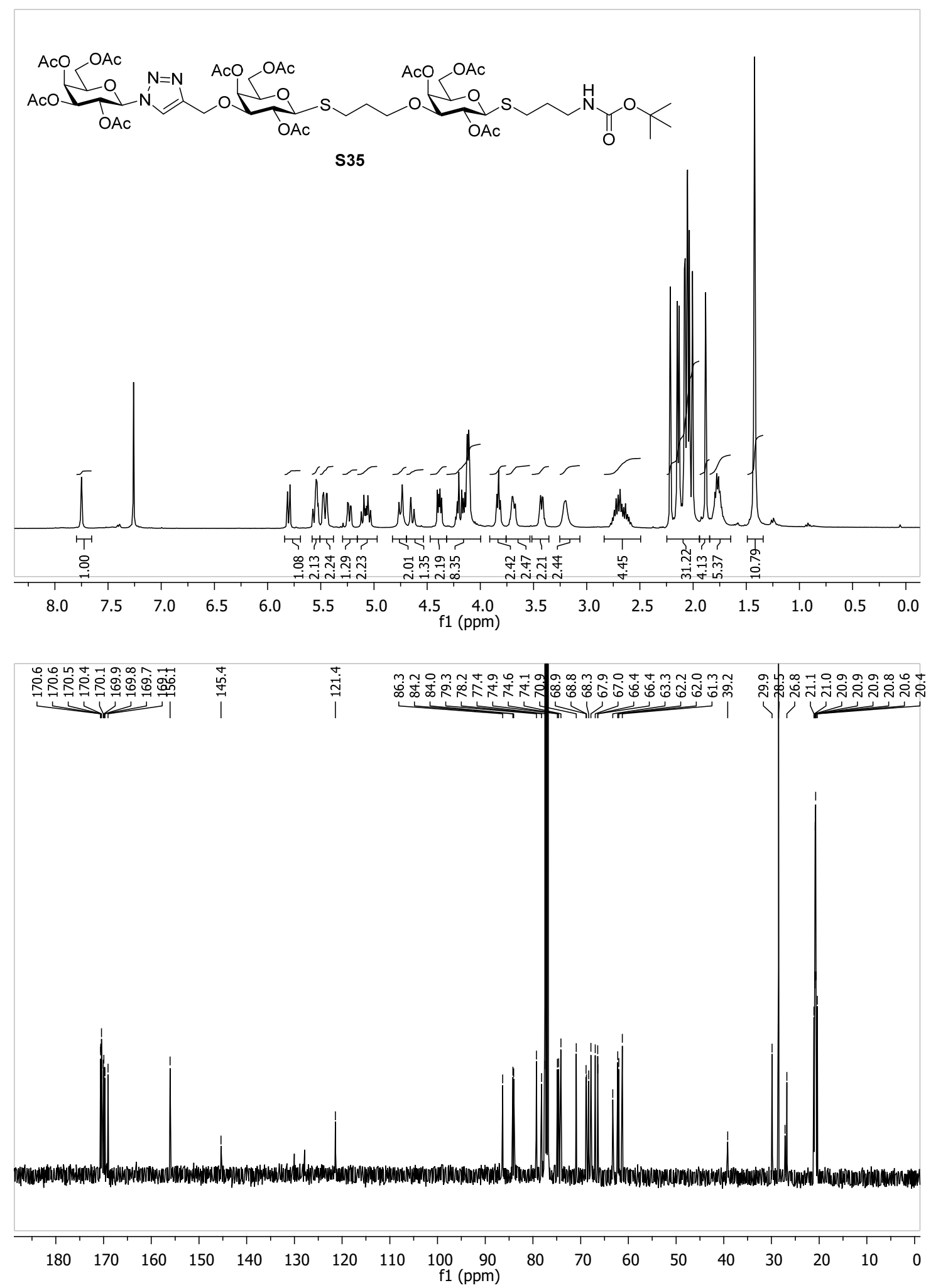

[(2",3",4",6"-tetra-O-acetyl-1"-thio- $\beta$-D-galactopyranosyl)propyl]- $\beta$-D-glucopyranosyl)-1H-[1,2,3]-triazol-4-ylmethyl]- $\beta$-D-galactopyranoside S36
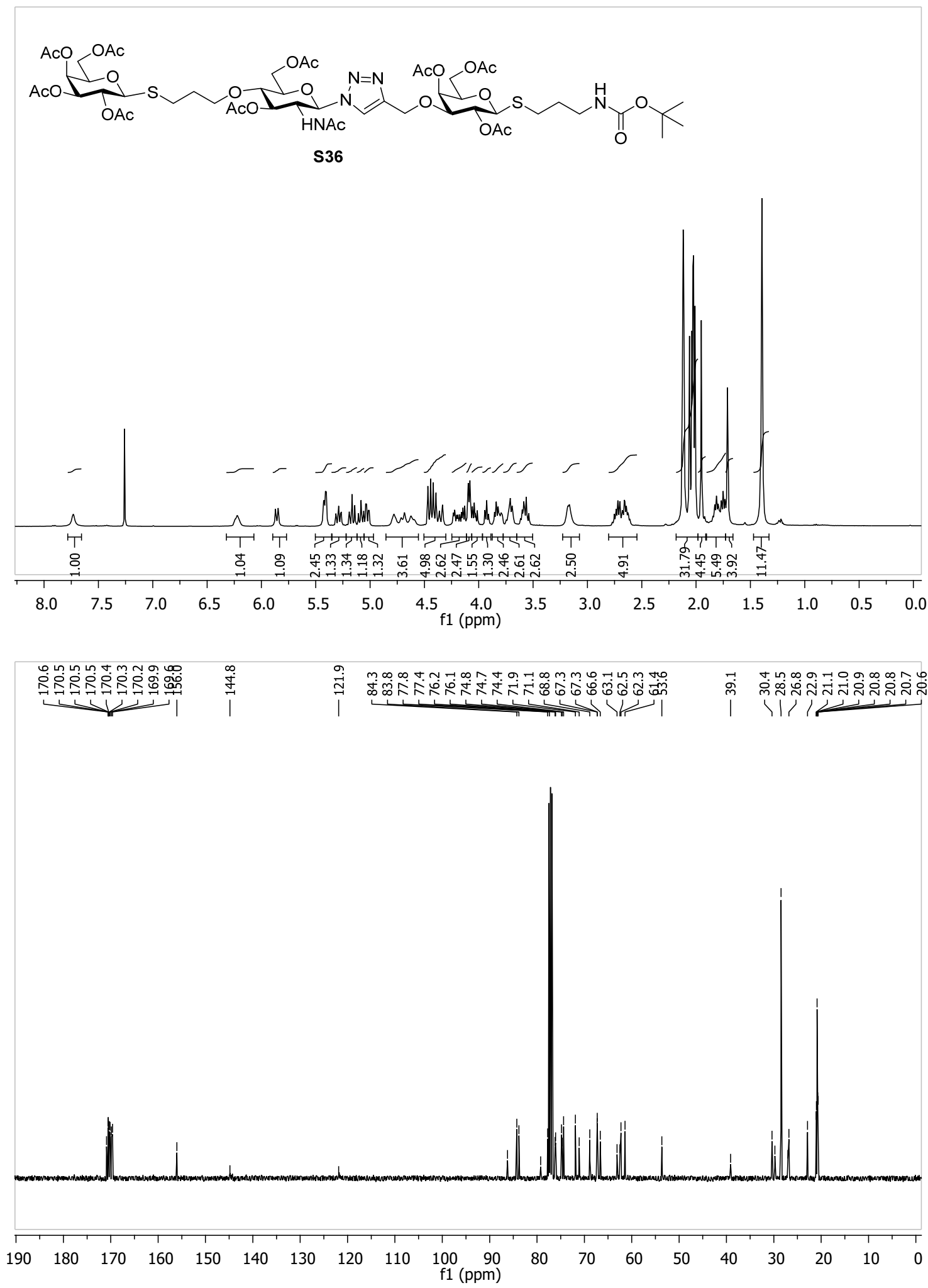
1-(tButyl(3-mercaptopropyl)carbamate)

2,4,6-tri-O-acetyl-3-O-[(2',4',6'-tri-O-acetyl-3'-O-[(2",3",4",6"'-tetra-Oacetyl- $\alpha$-D-mannopyranosyl)-1H-[1,2,3]-triazol-4-yl-methyl]-1'-thio- $\beta$-D-galactopyranosyl)propyl]- $\beta$-D-

galactopyranoside $\mathbf{S 3 7}$
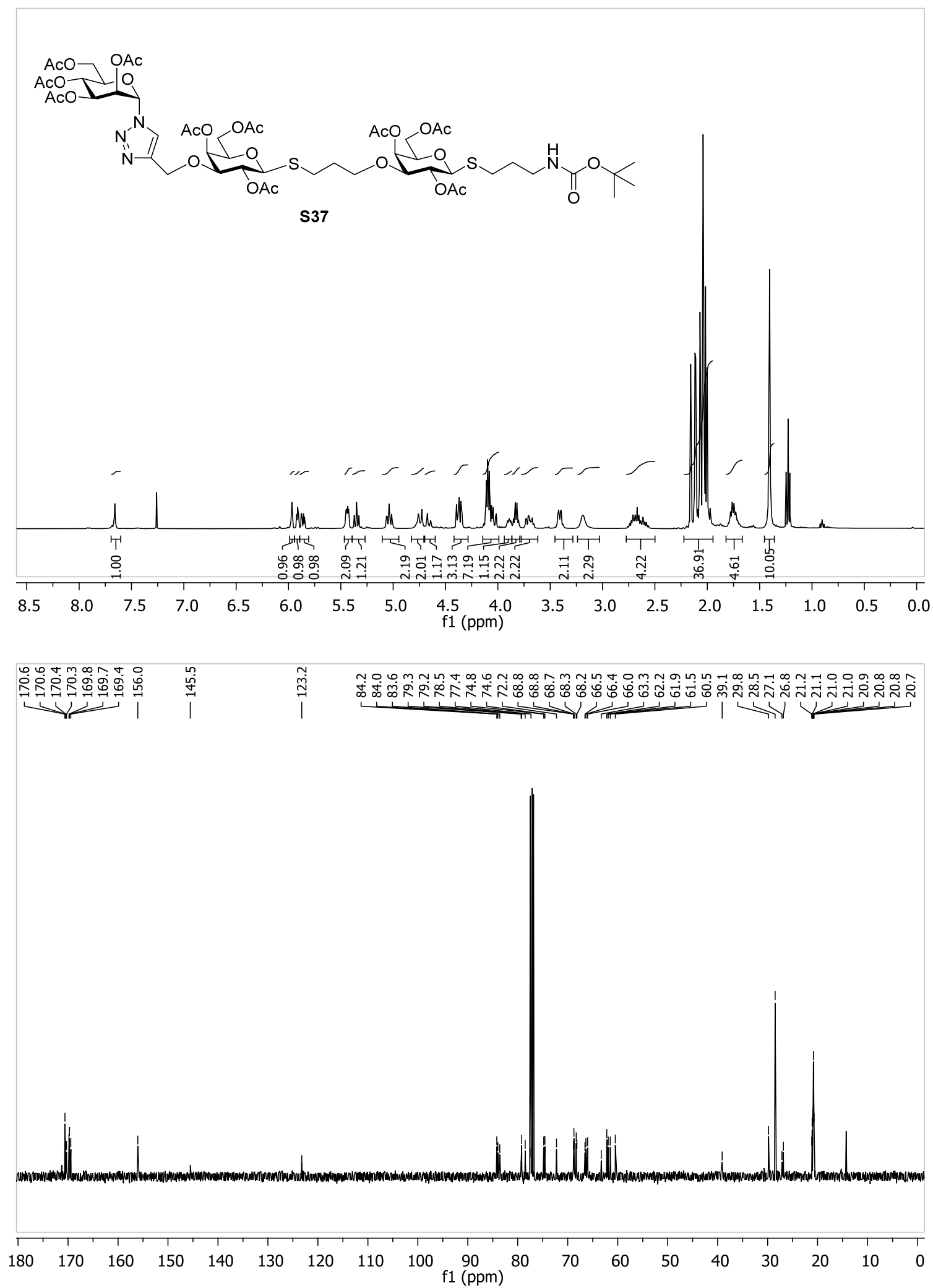
1-(tButyl(3-mercaptopropyl)carbamate) ～2,4,6-tri-O-acetyl-3-O-[(2',4',6'-O-acetyl-3'-O-[(2"-acetamido-2"-deoxy-

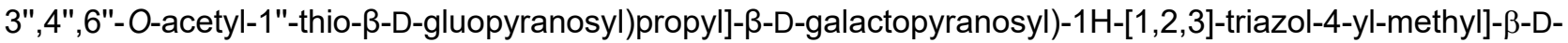
galactopyranoside $\mathbf{S} 38$
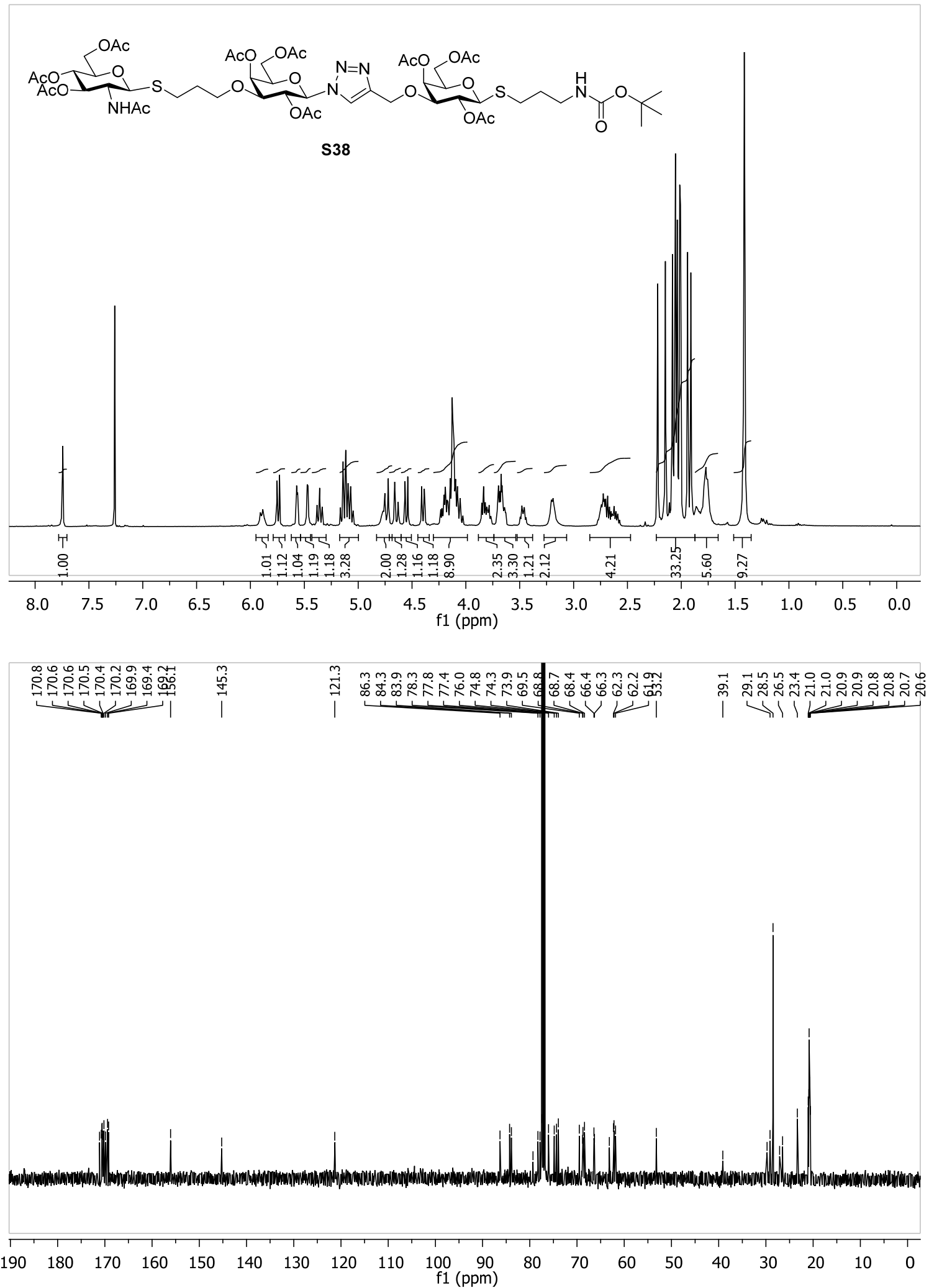
1-(tButyl(3-mercaptopropyl)carbamate) ～2,4,6-tri-O-acetyl-3-O-[(2',4',6'-O-acetyl-3'-O-[(2",3",4",6"-tetra-O-acetyl1 "-thio- $\alpha$-D-mannopyranosyl)propyl]- $\beta$-D-galactopyranosyl)-1H-[1,2,3]-triazol-4-yl-methyl]- $\beta$-D-galactopyranoside S39
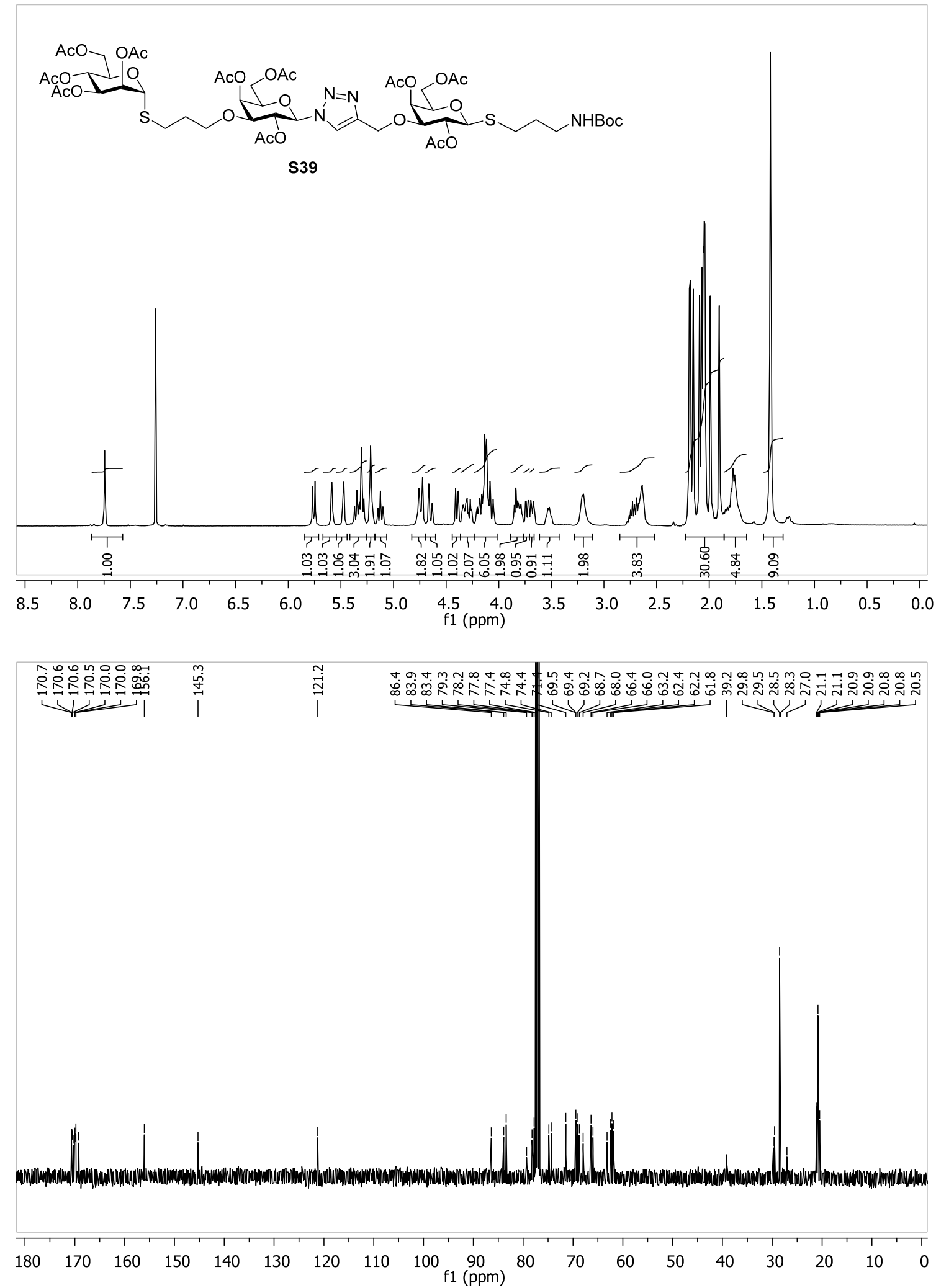
1-(tButyl(3-mercaptopropyl)carbamate)

2,4,6-tri-O-acetyl-3-O-[(2'-acetamido-2'-deoxy-3',6'-O-acetyl-4'-O[(2",3",4",6"-tetra-O-acetyl-1"-thio- $\alpha$-D-mannopyranosyl)propyl]- $\beta$-D-glucopyranosyl)-1H-[1,2,3]-triazol-4-ylmethyl]- $\beta$-D-galactopyranoside S40
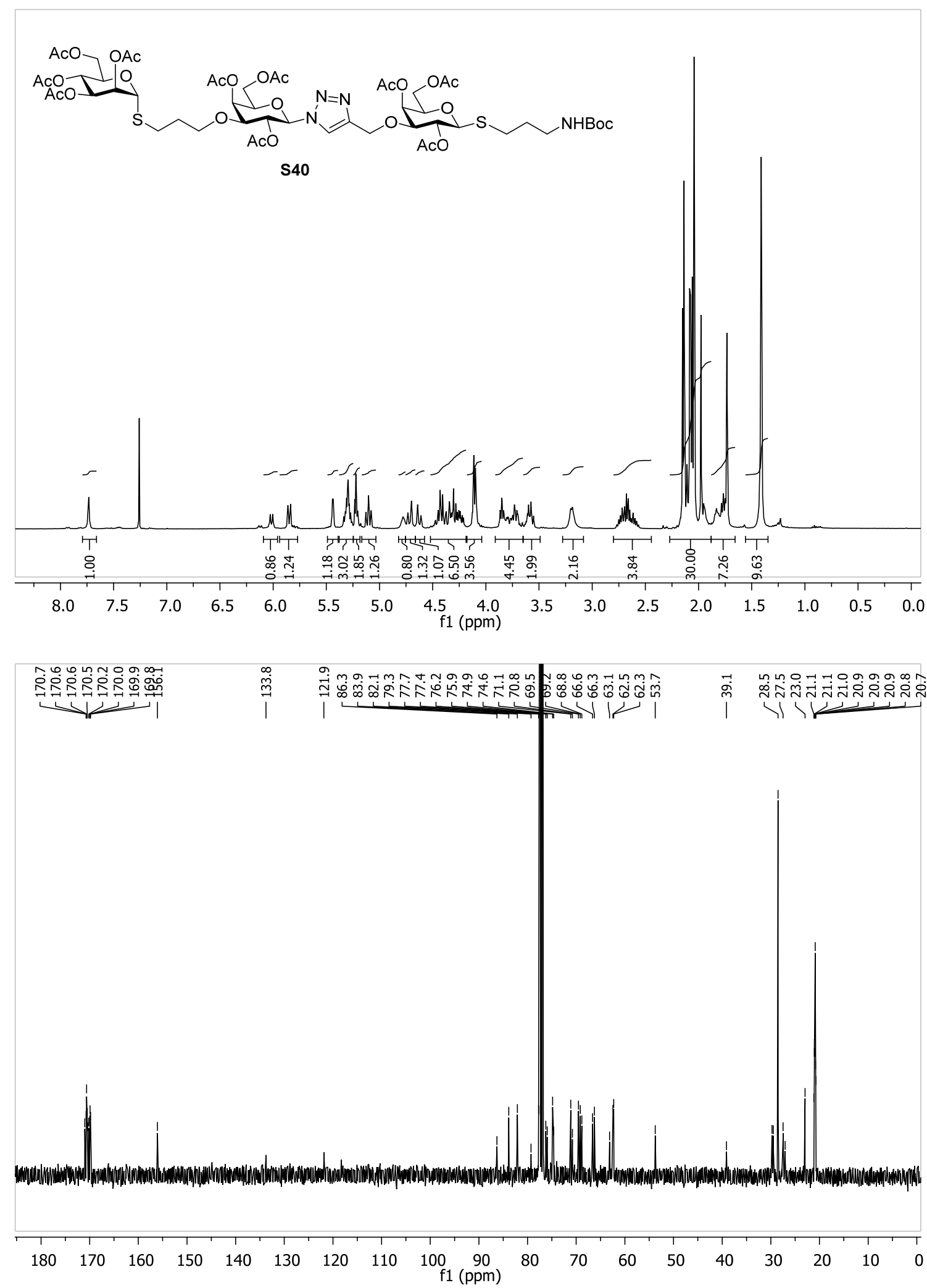
1-(tButyl(3-mercaptopropyl)carbamate) 2,4,6-tri-O-acetyl-3-O-[(2'-acetamido-2'-deoxy-3',6'-O-acetyl-4'-O-[(2"acetamido-2"-deoxy-3",4",6"-O-acetyl-1"-thio- $\beta$-D-glucopyranosyl)propyl]- $\beta$-D-glucopyranosyl)-1H-[1,2,3]-triazol4-yl-methyl]- $\beta$-D-galactopyranoside S41
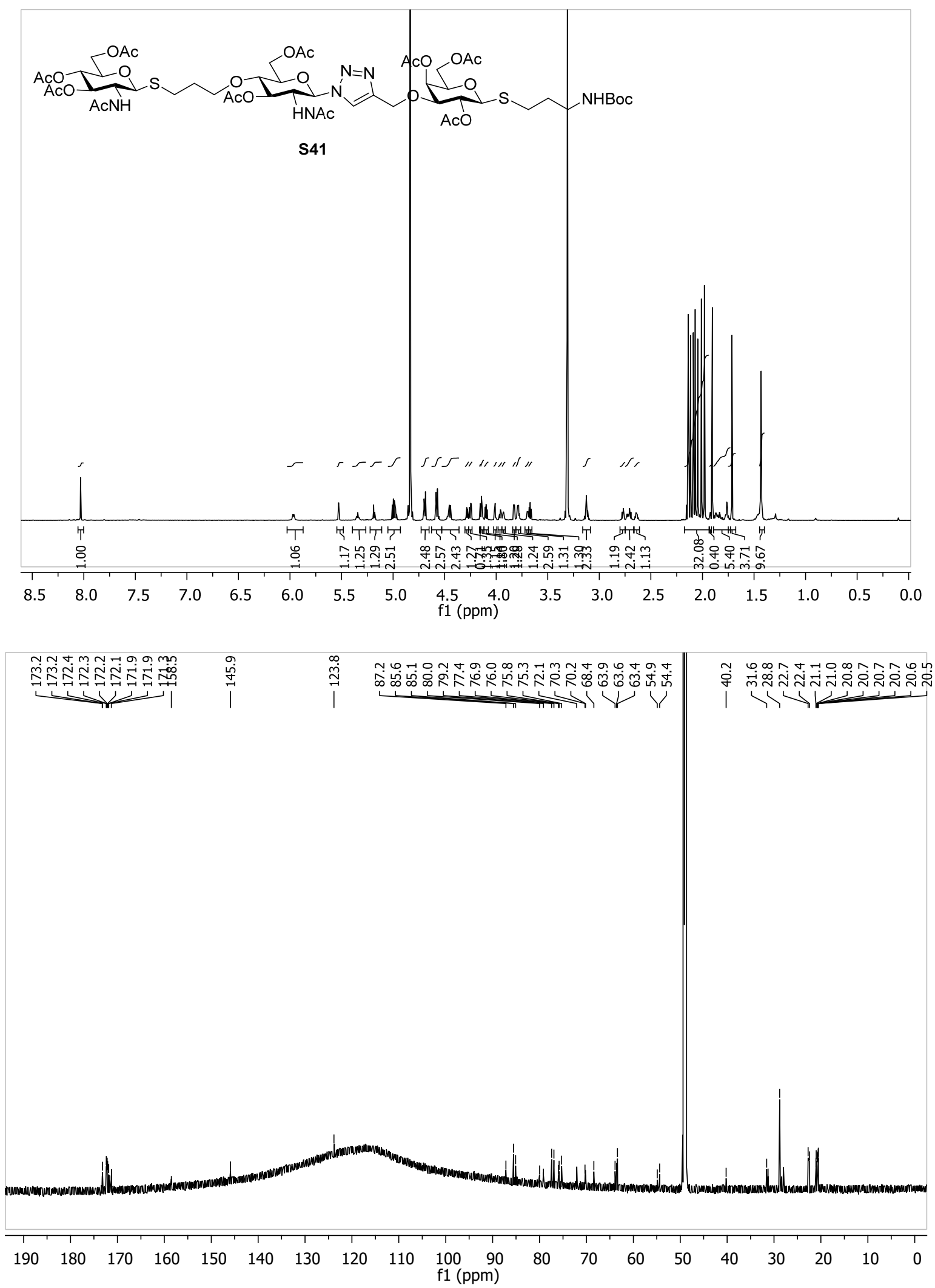
1-(tButyl(3-mercaptopropyl)carbamate) 2,4,6-tri-O-acetyl-3-O-[(3',4',6'-O-acetyl-2'-O-[(2",3",4",6"-tetra-O-acetyl1 "thio- $\alpha$-D-mannopyranosyl)propyl]- $\alpha$-D-mannopyranosyl)-1H-[1,2,3]-triazol-4-yl-methyl]- $\beta$-D-galactopyranoside

\section{S42}
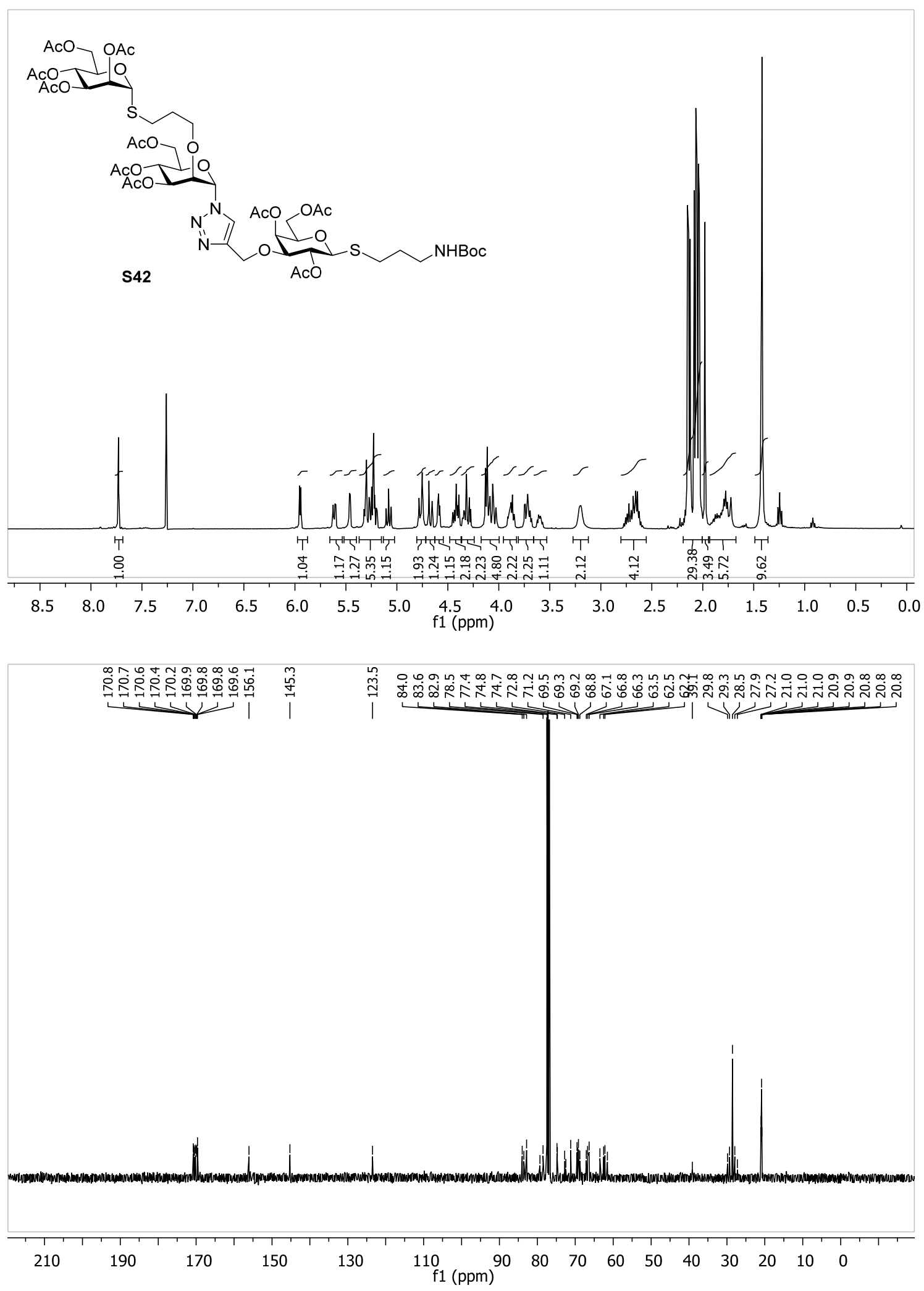
1-(tButyl(3-mercaptopropyl)carbamate) 2,4,6-tri-O-acetyl-3-O-[(2',4',6'-O-acetyl-3'-O-[(2",4",6"-tri-O-acetyl-3"-O[(2"',3"',4"',6"'-tetra-O-acetyl-1"'-thio- $\beta$-D-galactopyranosyl)propyl]- $\beta$-D-galactopyranosyl)-1H-[1,2,3]-triazol-4-ylmethyl]-1'-thio- $\beta$-D-galactopyranosyl)propyl]- $\beta$-D-galactopyranoside $\mathbf{S 4 3}$
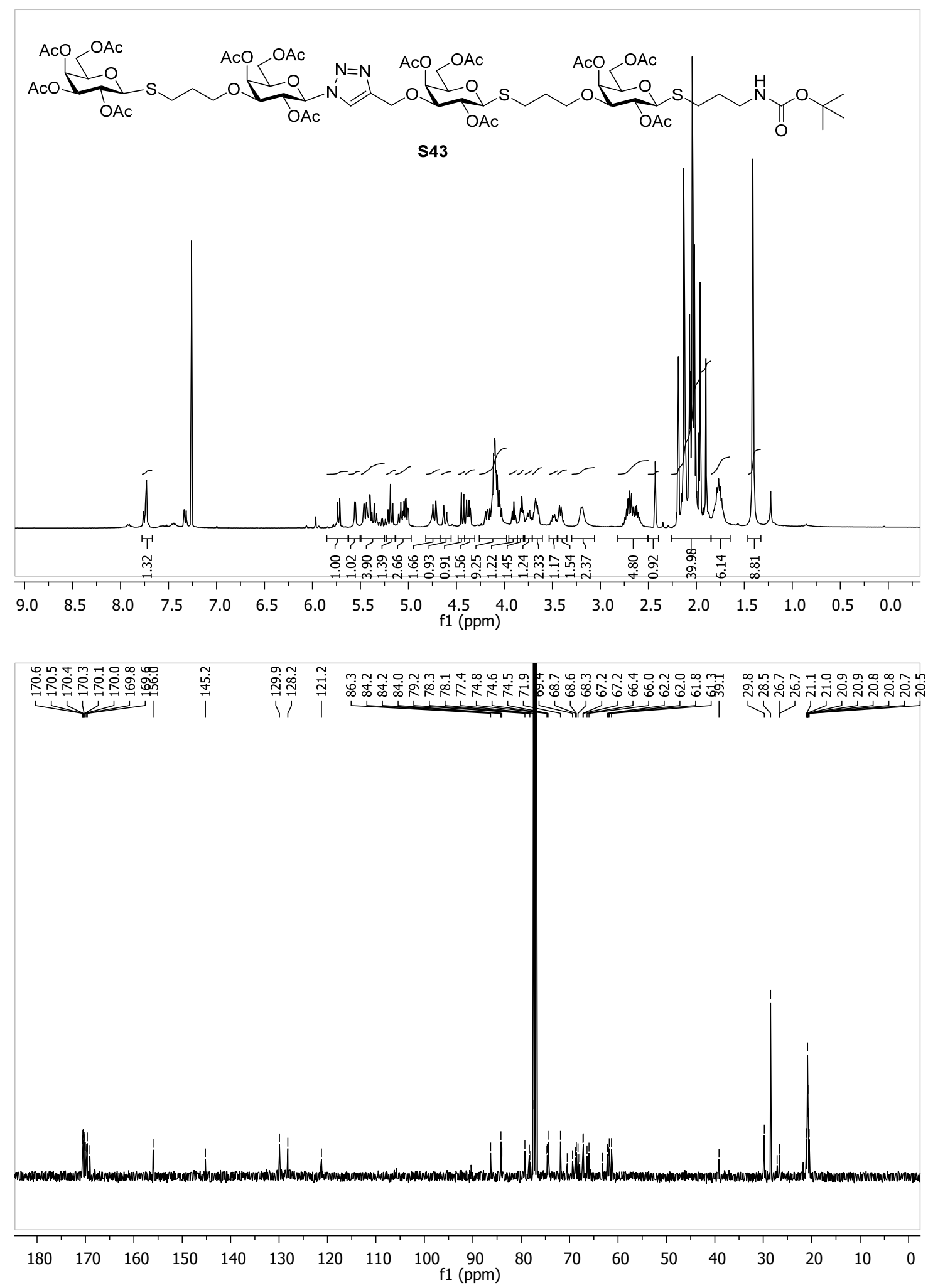
1-(tButyl(3-mercaptopropyl)carbamate) 2,4,6-tri-O-acetyl-3-O-[(2',4',6'-O-acetyl-3'-O-[(2",4",6"-tri-O-acetyl-3"-O[(2"',3'",4"',6"'-tetra-O-acetyl- $\beta$-D-galactopyranosyl)-1H-[1,2,3]-triazol-4-yl-methyl]-1"-thio- $\beta$-D-

galactopyranosyl)propyl]- $\beta$-D-galactopyranosyl)-1H-[1,2,3]-triazol-4-yl-methyl]- $\beta$-D-galactopyranoside S44

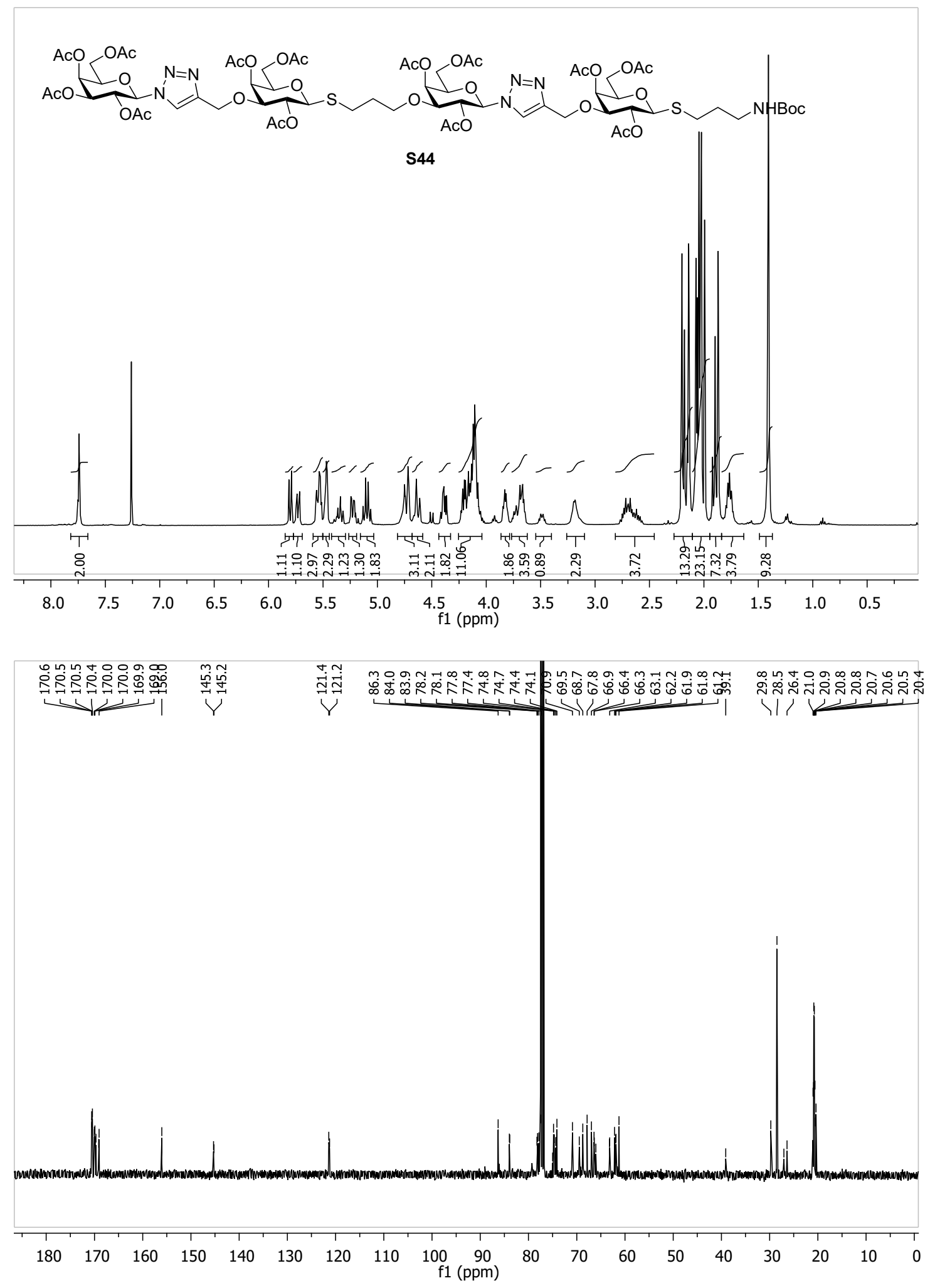



[(2",4",6"-tri-O-acetyl-3"-O-[(2"',3"',4"',6"'-tetra-O-acetyl- $\alpha-D-m a n n o p y r a n o s y l)-1 \mathrm{H}-[1,2,3]-t r i a z o l-4-y l-m e t h y l]-1 "-$ thio- $\beta$-D-galactopyranosyl)propyl]- $\beta$-D-glucopyranosyl)-1H-[1,2,3]-triazol-4-yl-methyl]- $\beta$-D-galactopyranoside $\mathbf{S 4 5}$
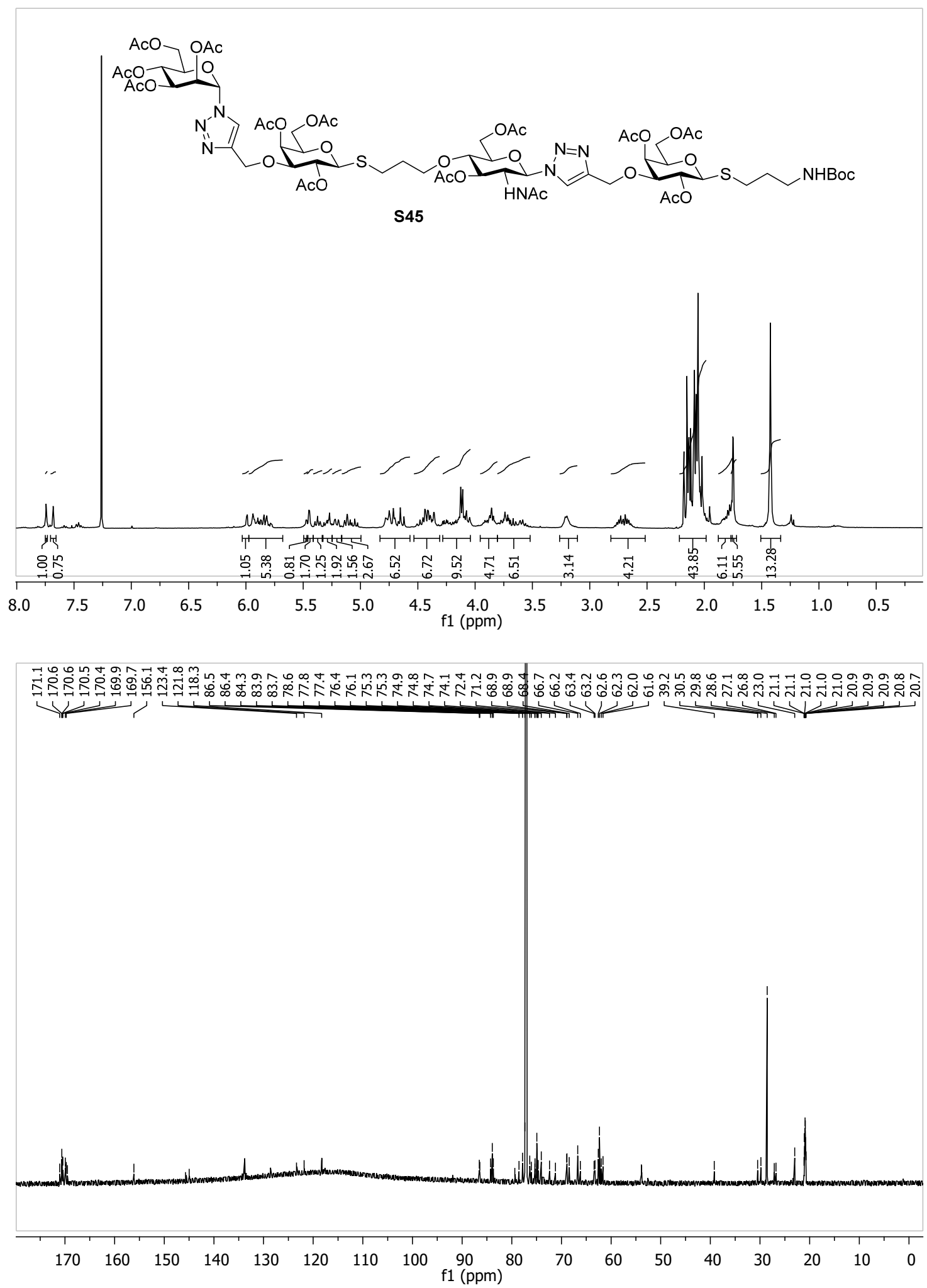


\section{Deprotected glycan mimics}

1-(tButyl(3-mercaptopropyl)carbamate) 3-O-[(3'-O-[(1"-thio- $\beta$-D-galactopyranosyl)propyl]- $\beta$-D-galactopyranosyl)$1 \mathrm{H}$-[1,2,3]-triazol-4-yl-methyl]- $\beta$-D-galactopyranoside $\mathbf{S 4 6}$
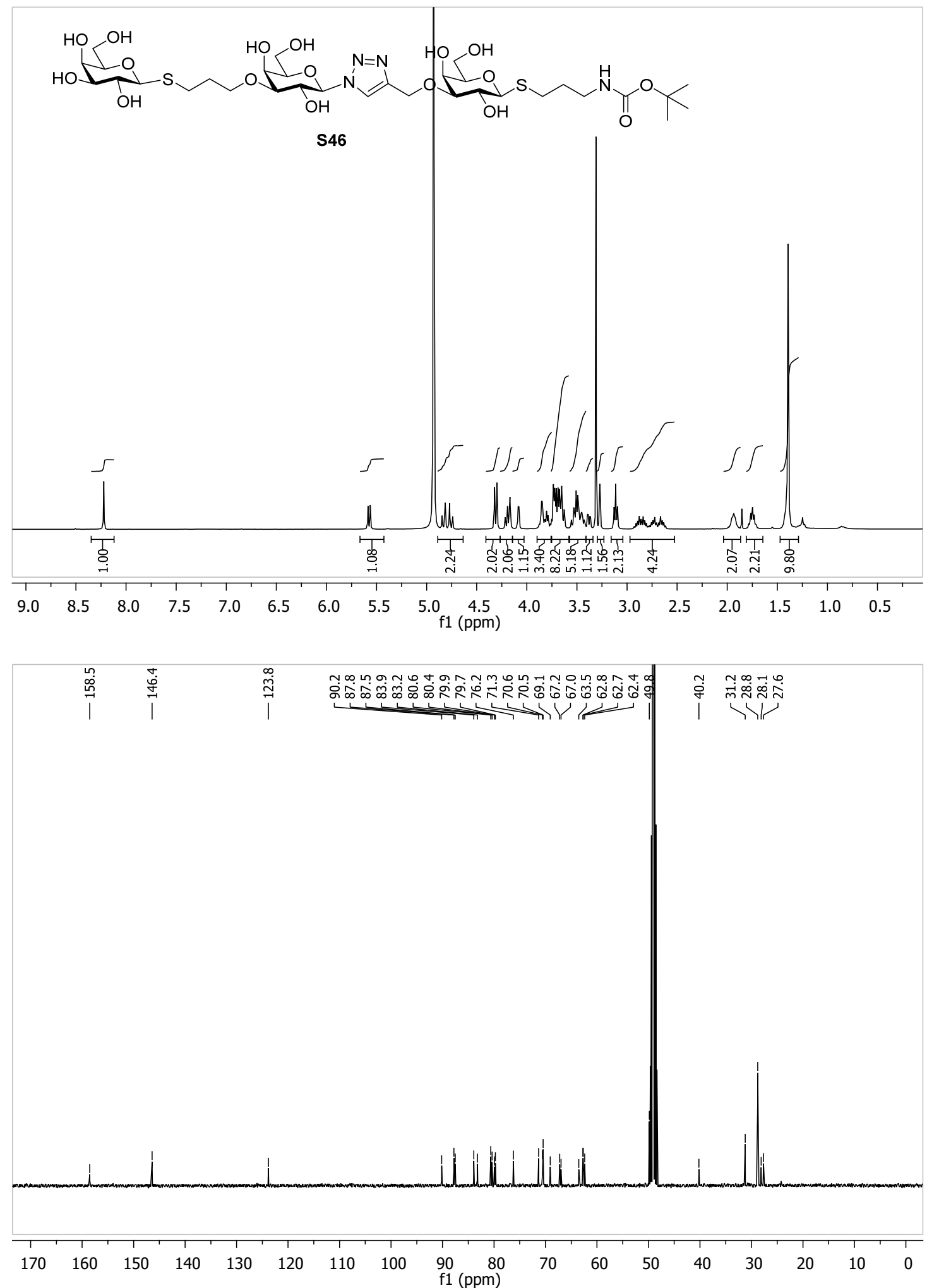
1-(tButyl(3-mercaptopropyl)carbamate) 3-O-[(3'-O-[( $\beta$-D-galactopyranosyl)-1H-[1,2,3]-triazol-4-yl-methyl]-1'-thio- $\beta$ D-galactopyranosyl)propyl]- $\beta$-D-galactopyranoside S47
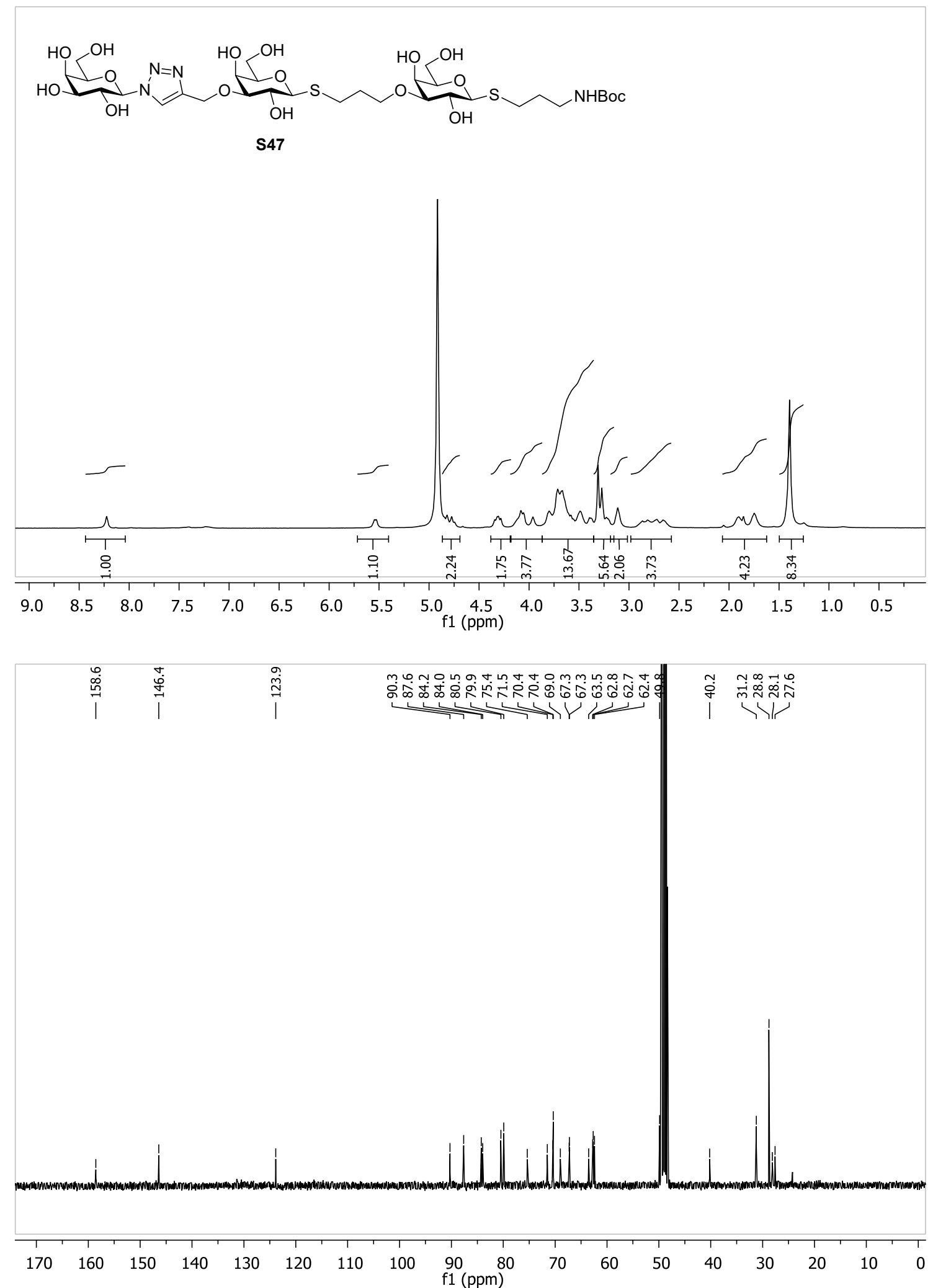
1-(tButyl(3-mercaptopropyl)carbamate 3-O-[(2'-acetamido-2'-deoxy -4'-O-[(1"-thio- $\beta$-D-galactopyranosyl)propyl]- $\beta$ D-glucopyranosyl)-1H-[1,2,3]-triazol-4-yl-methyl]- $\beta$-D-galactopyranoside S48
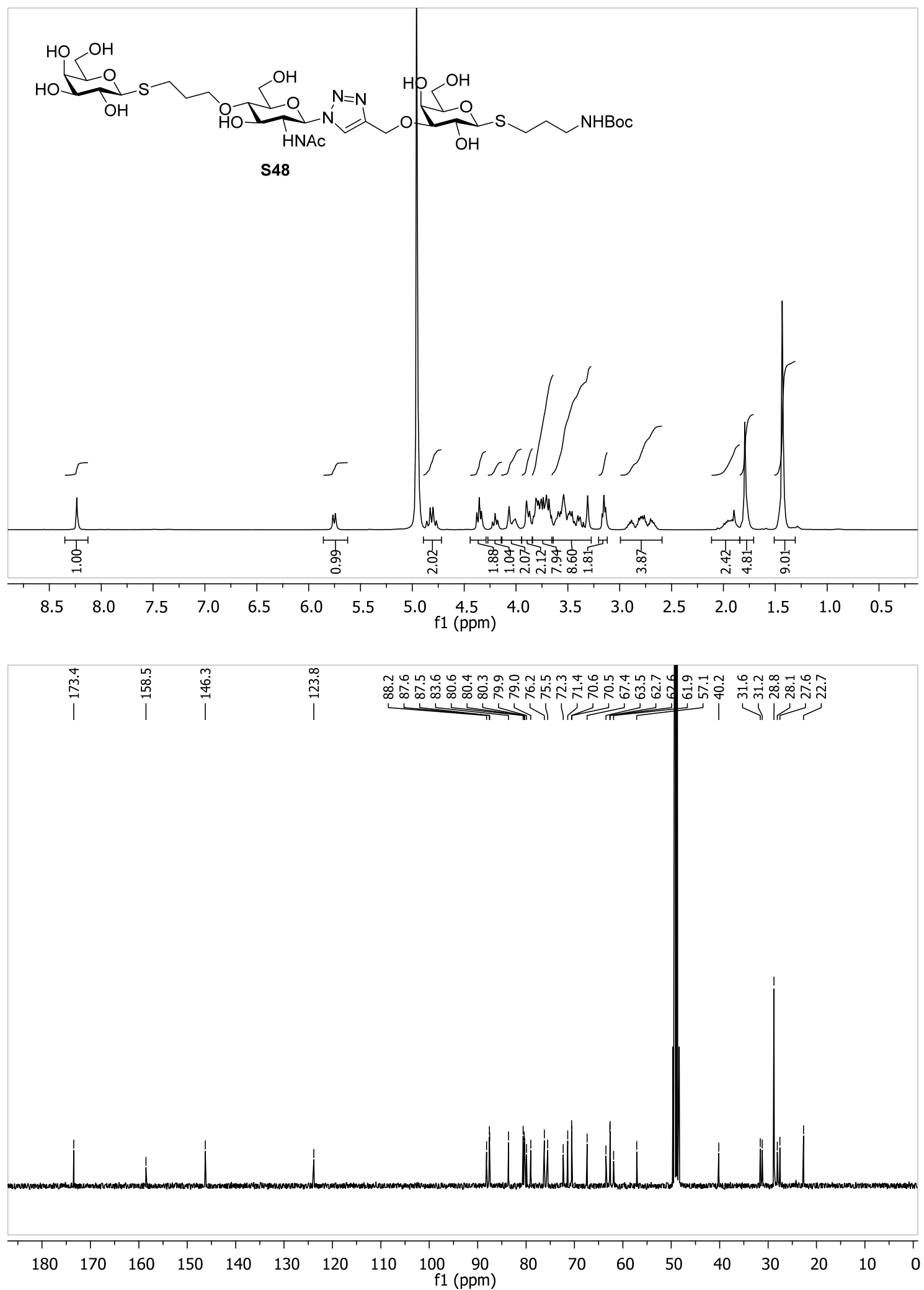
1-(tButyl(3-mercaptopropyl)carbamate) 3-O-[(-3'-O-[(- $\alpha$-D-mannopyranosyl)-1H-[1,2,3]-triazol-4-yl-methyl]-1'-thio$\beta$-D-galactopyranosyl)propyl]- $\beta$-D-galactopyranoside S49
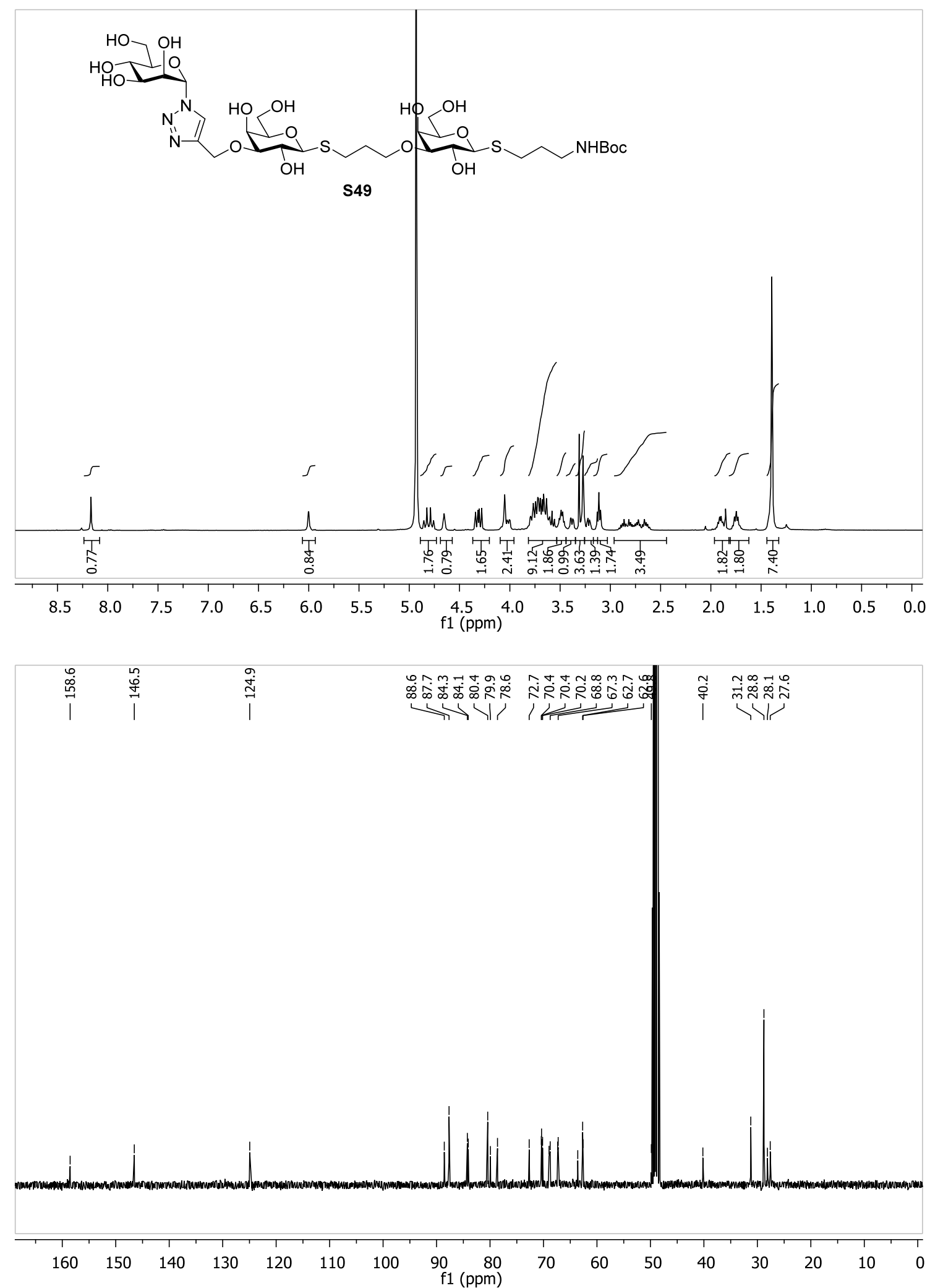
1-(tButyl(3-mercaptopropyl)carbamate 3-O-[(3'-O-[(2"-acetamido-2"-deoxy -1"-thio- $\beta$-D-gluopyranosyl)propyl]- $\beta$ D-galactopyranosyl)-1H-[1,2,3]-triazol-4-yl-methyl]- $\beta$-D-galactopyranoside S50
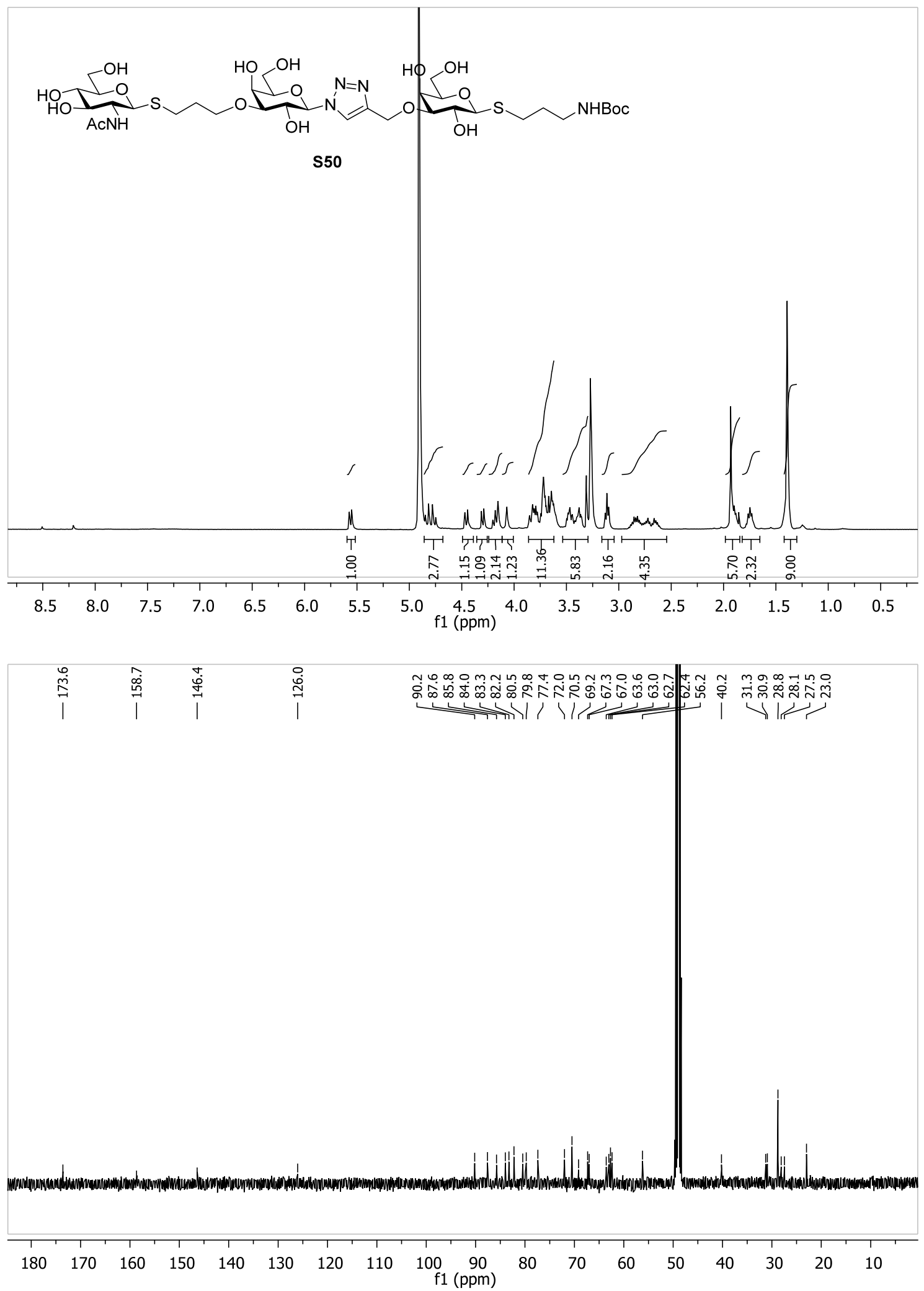
1-(tButyl(3-mercaptopropyl)carbamate) 3-O-[(3'-O-[(1"-thio- $\alpha$-D-mannopyranosyl)propyl]- $\beta$-D-galactopyranosyl)$1 \mathrm{H}-[1,2,3]-$ triazol-4-yl-methyl]- $\beta$-D-galactopyranoside $\mathbf{S 5 1}$
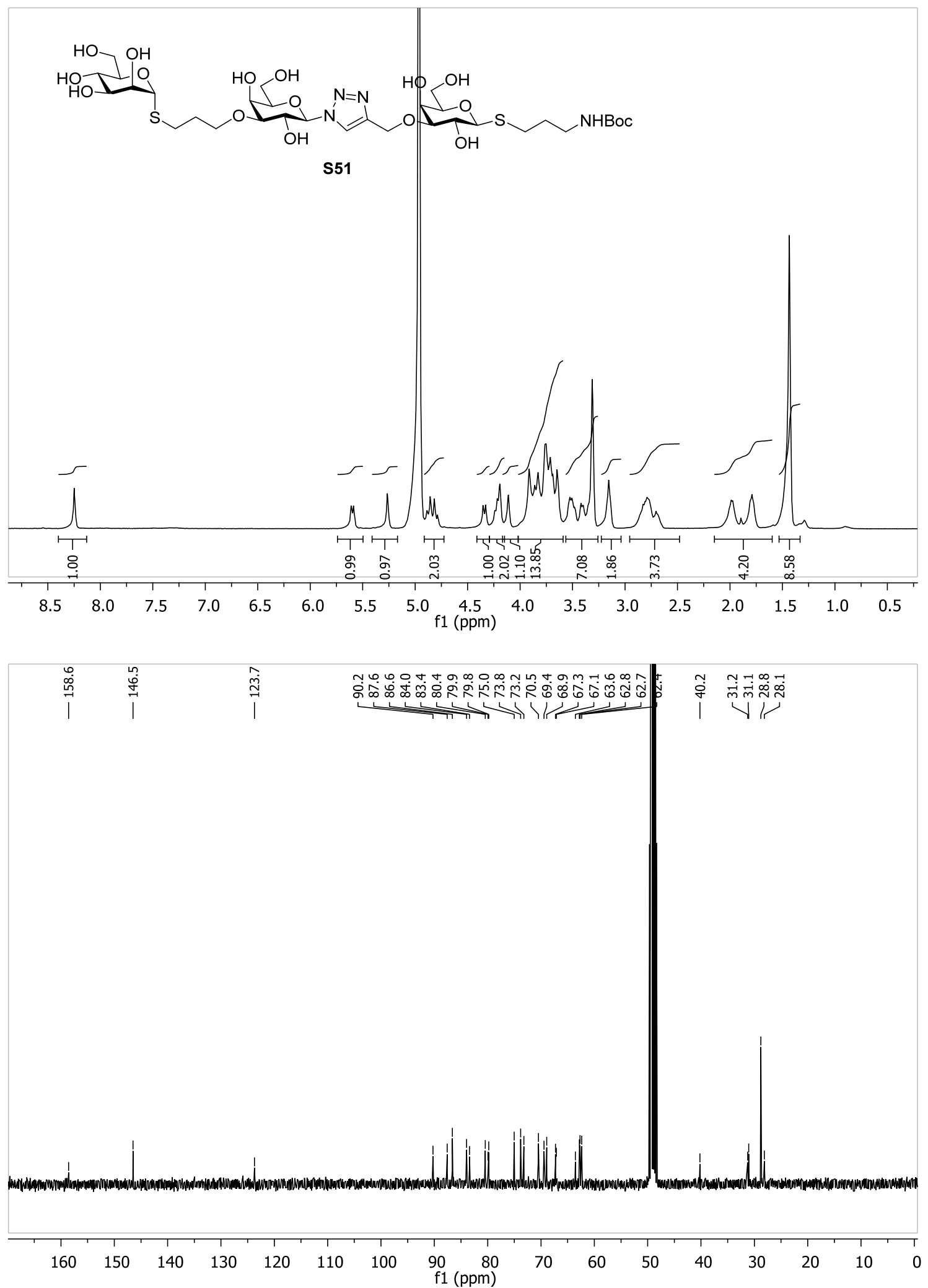
1-(tButyl(3-mercaptopropyl)carbamate) 3-O-[(2'-acetamido-2'-deoxy-4'-O-[(1"-thio- $\alpha$-D-mannopyranosyl)propyl]- $\beta$ D-glucopyranosyl)-1H-[1,2,3]-triazol-4-yl-methyl]- $\beta$-D-galactopyranoside S52
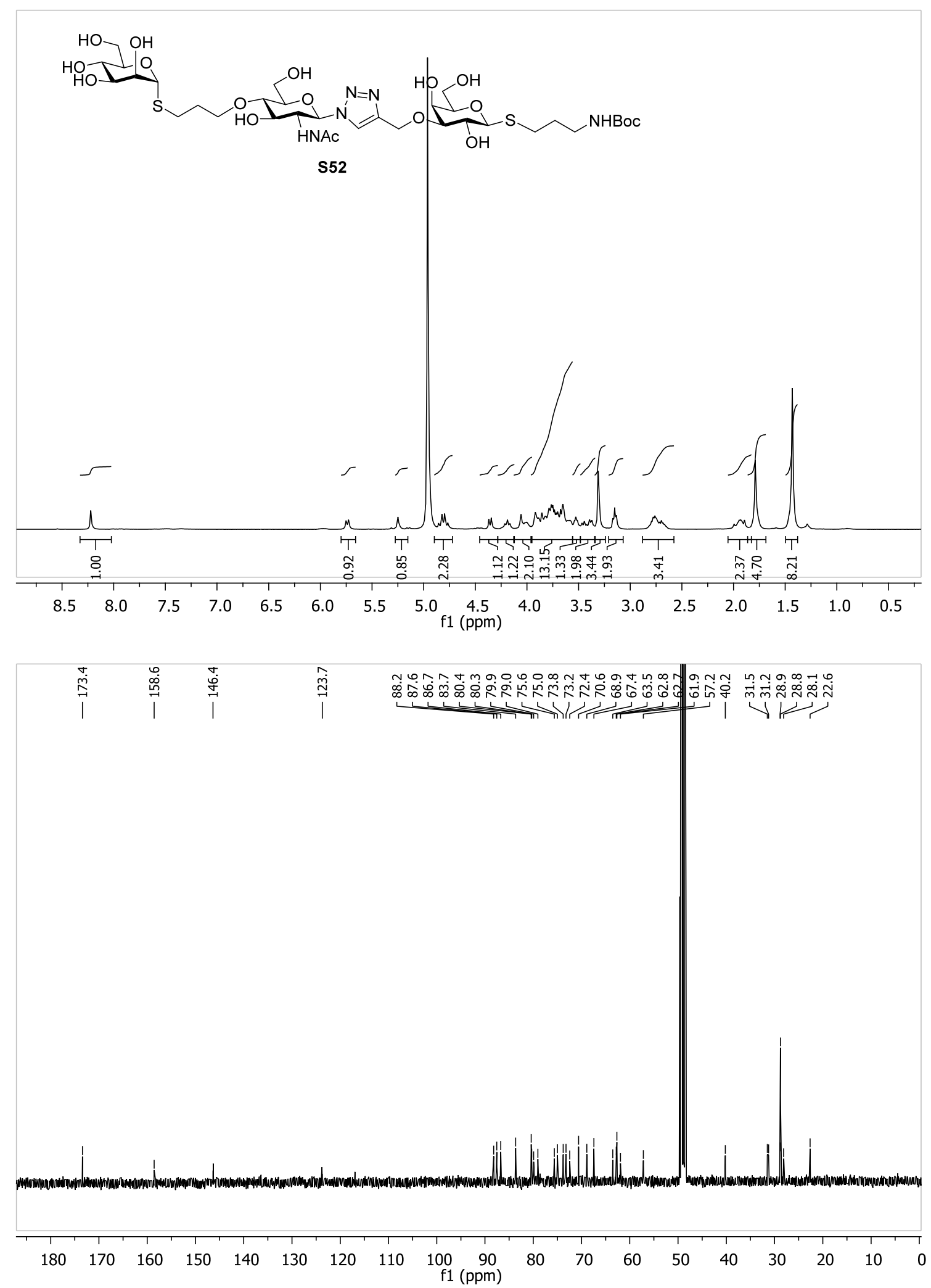
1-(tButyl(3-mercaptopropyl)carbamate) 3-O-[(2'-acetamido-2'-deoxy -4'-O-[(2"-acetamido-2"-deoxy -1"-thio-ß-Dglucopyranosyl)propyl]- $\beta$-D-glucopyranosyl)-1H-[1,2,3]-triazol-4-yl-methyl]- $\beta$-D-galactopyranoside S53
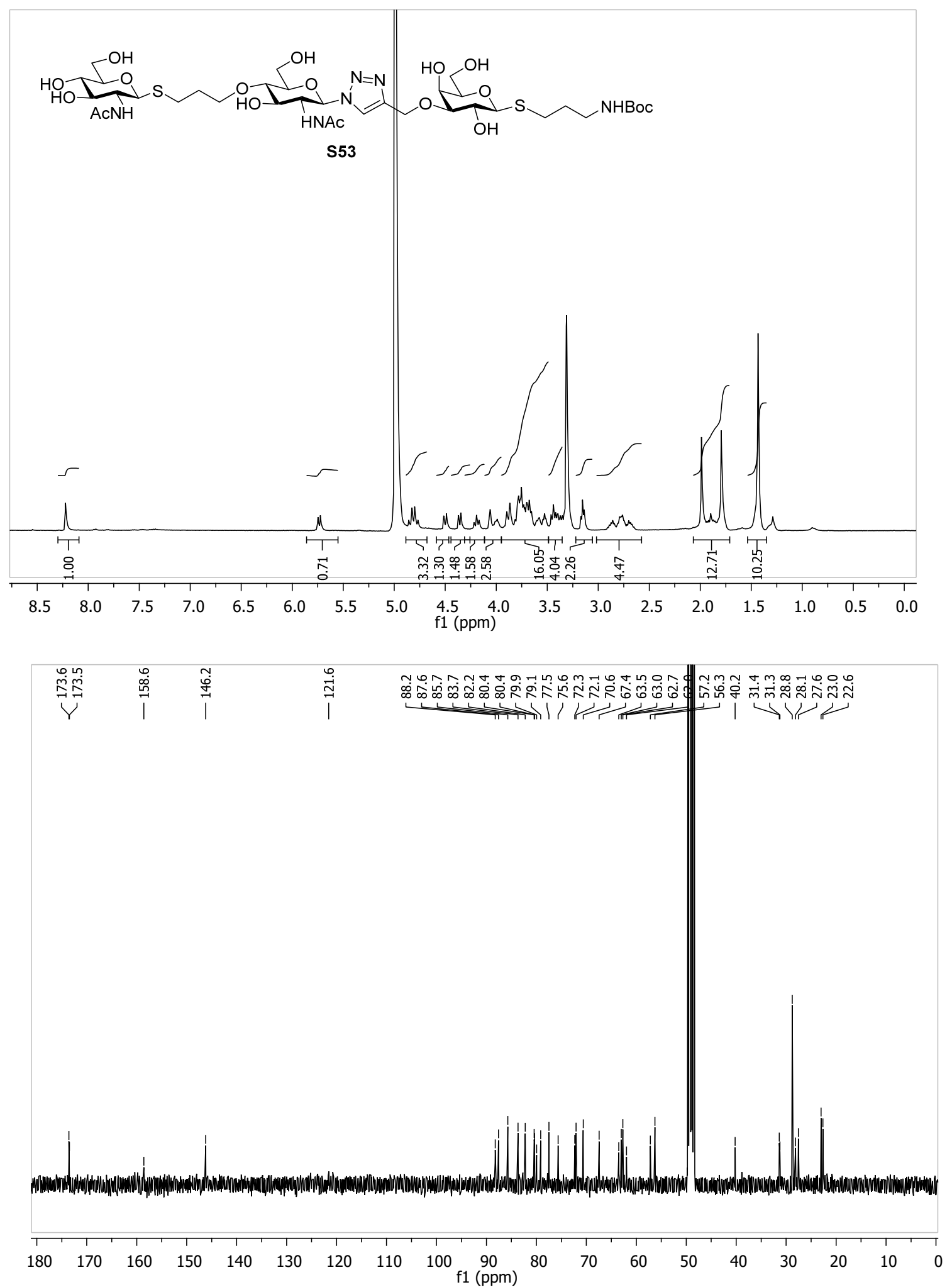
1-(tButyl(3-mercaptopropyl)carbamate) 3-O-[(2'-O-[(1"thio- $\alpha$-D-mannopyranosyl)propyl]- $\alpha$-D-mannopyranosyl)$1 \mathrm{H}-[1,2,3]$-triazol-4-yl-methyl]- $\beta$-D-galactopyranoside S54
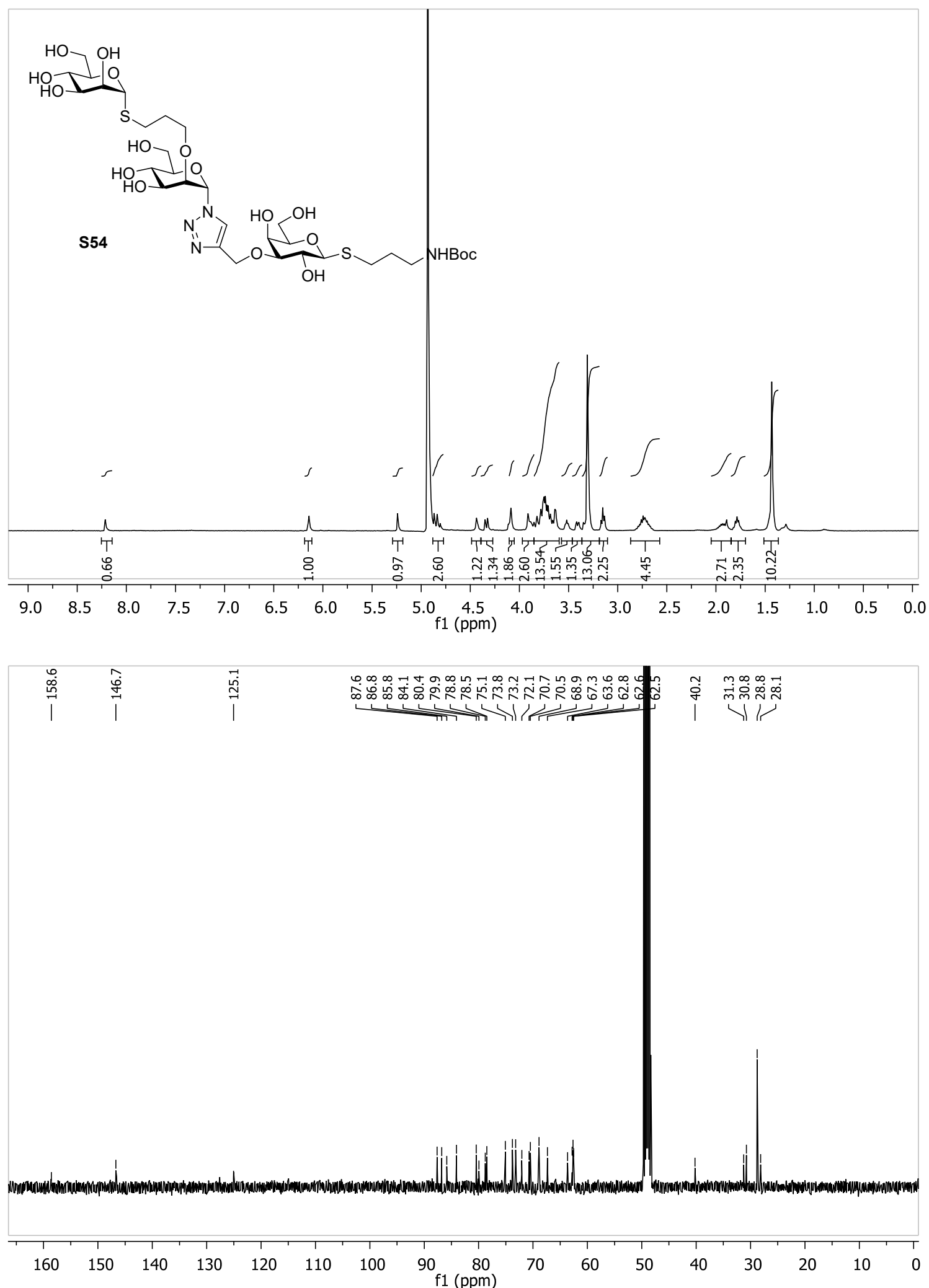
1-(tButyl(3-mercaptopropyl)carbamate) 3-O-[(3'-O-[(3"-O-[(1"'-thio- $\beta$-D-galactopyranosyl)propyl]- $\beta$-Dgalactopyranosyl)-1H-[1,2,3]-triazol-4-yl-methyl]-1'-thio- $\beta$-D-galactopyranosyl)propyl]- $\beta$-D-galactopyranoside S55
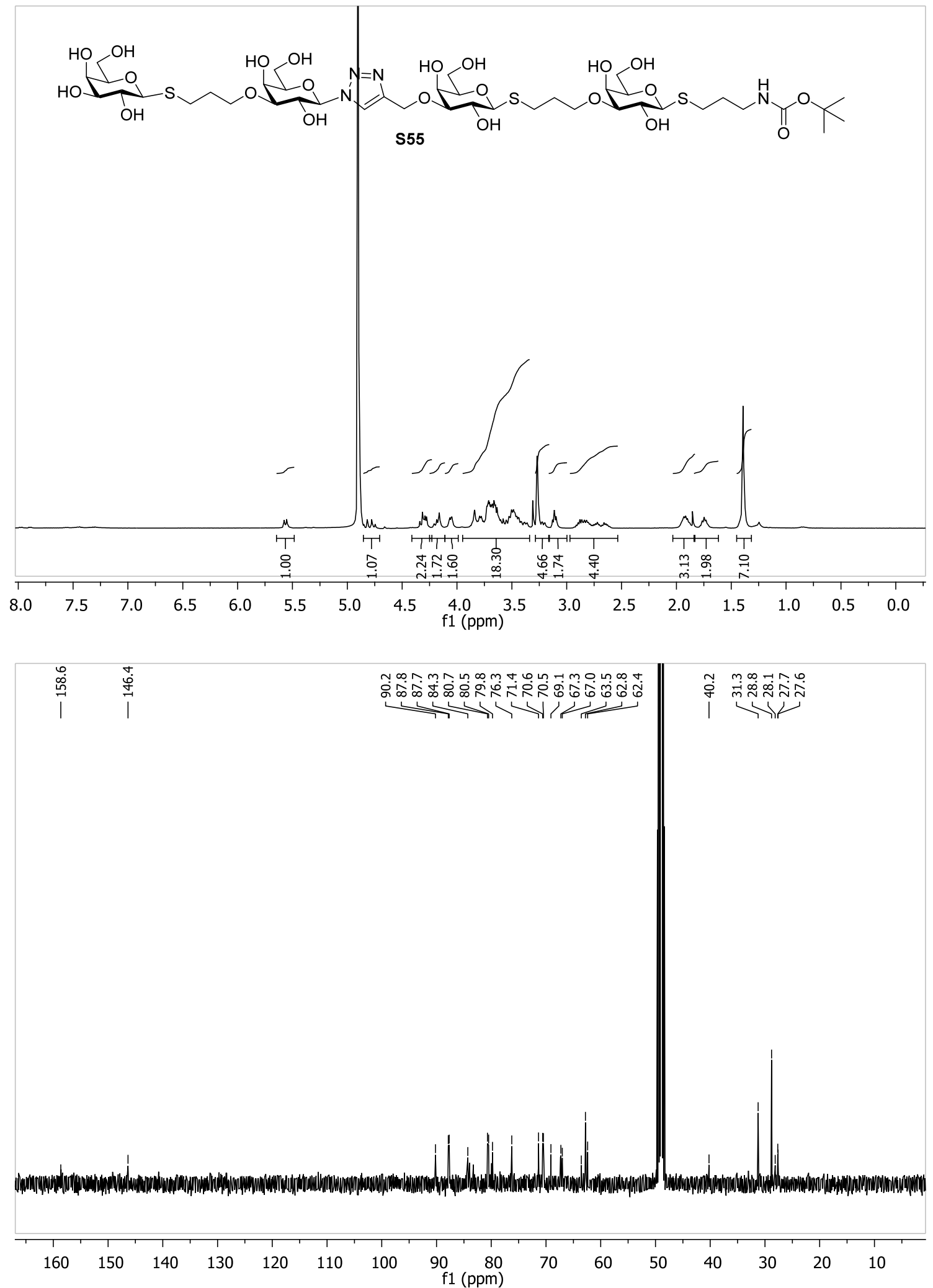
1-(tButyl(3-mercaptopropyl)carbamate) 3-O-[(-3'-O-[(-3"-O-[(- $\beta$-D-galactopyranosyl)-1H-[1,2,3]-triazol-4-ylmethyl]-1"-thio- $\beta$-D-galactopyranosyl)propyl]- $\beta$-D-galactopyranosyl)-1H-[1,2,3]-triazol-4-yl-methyl]- $\beta$-Dgalactopyranoside S56
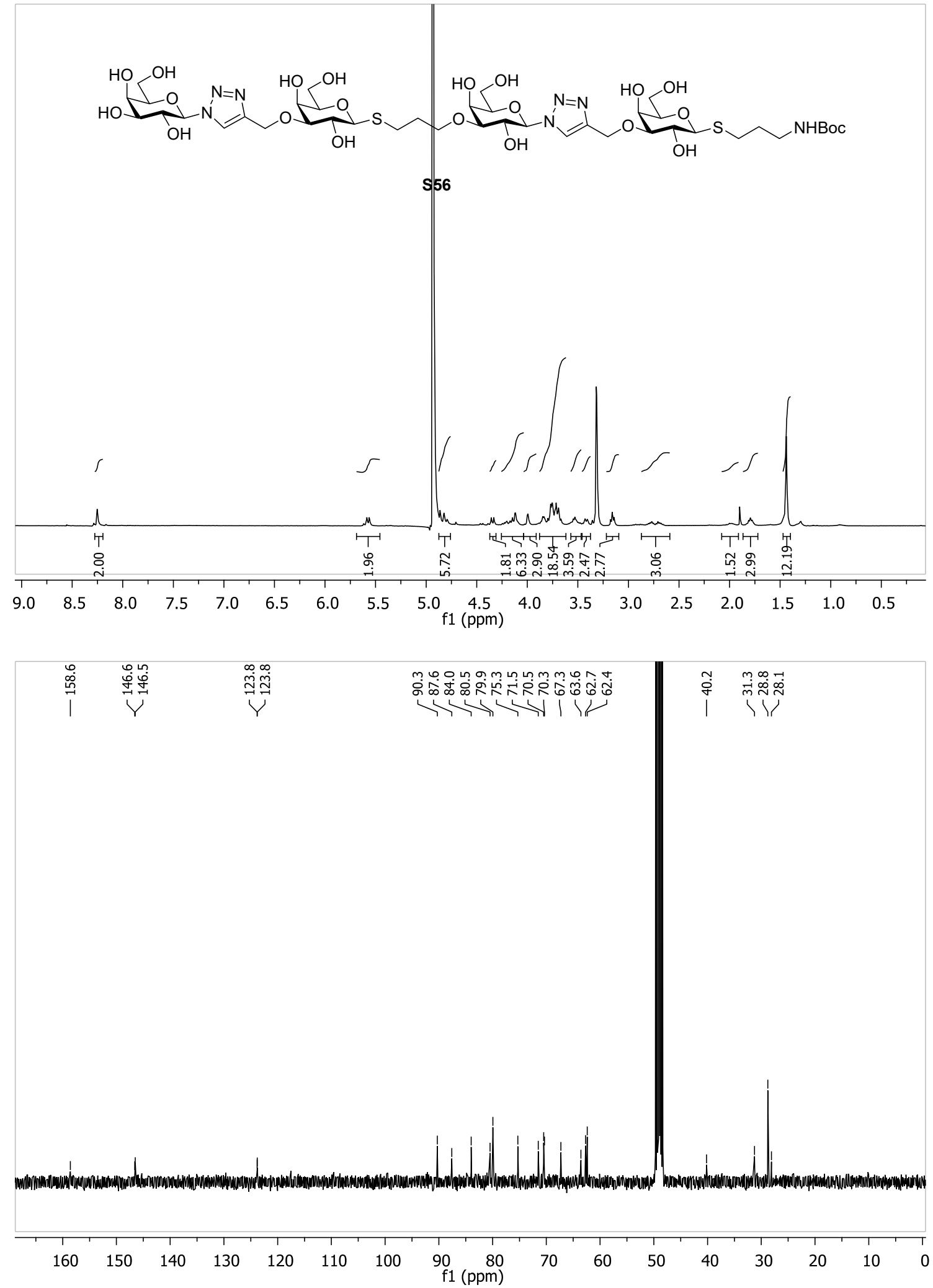
1-(tButyl(3-mercaptopropyl)carbamate) 3-O-[(2'-acetamido-2'-deoxy-4'-O- [(3"-O-[(- $\alpha$-D-mannopyranosyl)-1H[1,2,3]-triazol-4-yl-methyl]-1"-thio- $\beta$-D-galactopyranosyl)propyl]- $\beta$-D-glucopyranosyl)-1H-[1,2,3]-triazol-4-ylmethyl]- $\beta$-D-galactopyranoside S57
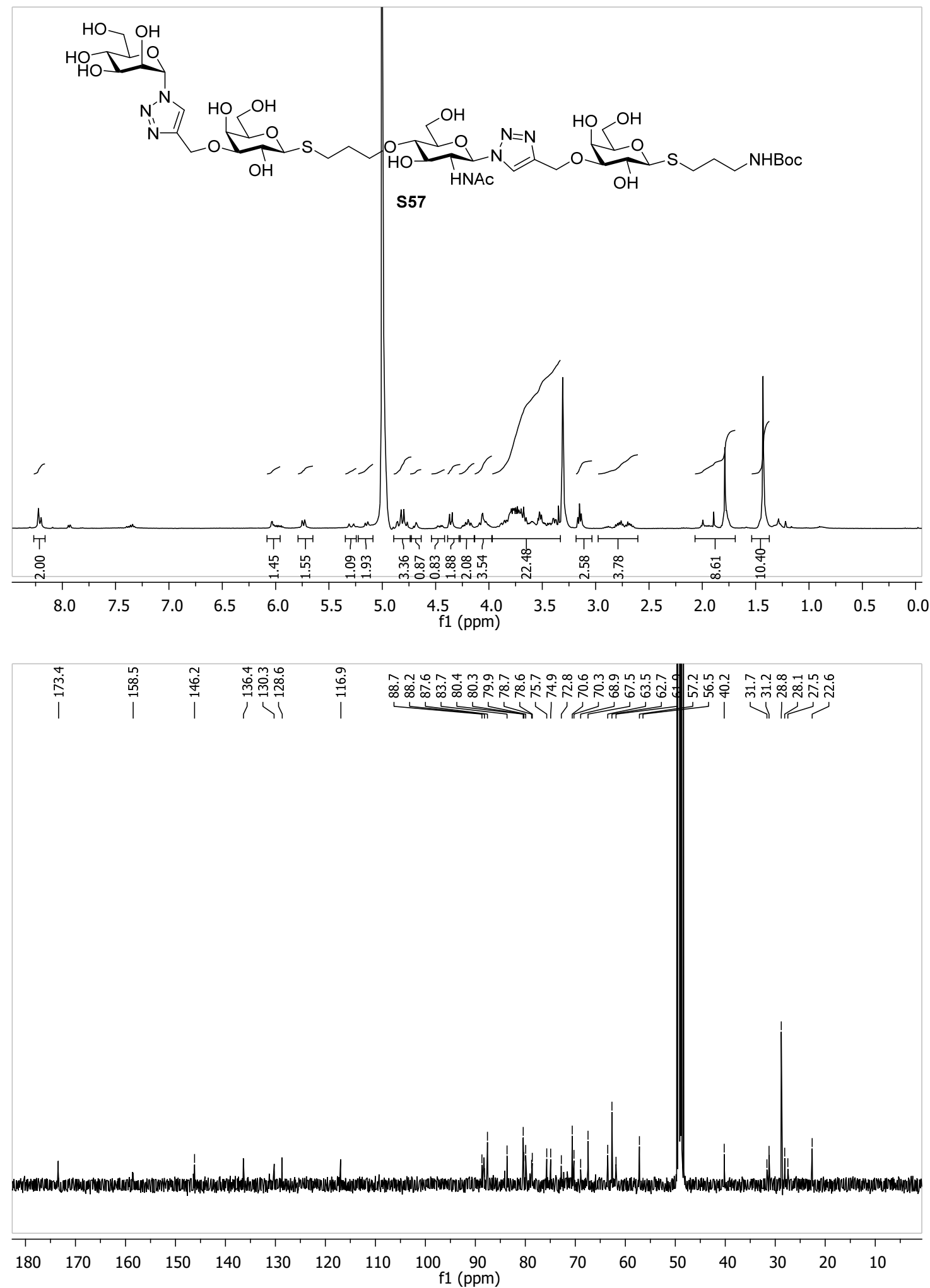


\section{References}

(1) O. Blixt, E. Cló, A. S. Nudelman, K. K. Sørensen, T. Clausen, H. H. Wandall, P. O. Livingston, H. Clausen, K. J. Jensen, J. Proteome Res. 2010, 9 , $5250-5261$.

(2) A. Janelle-Montcalm, C. Boileau, F. Poirier, J.-P. Pelletier, M. Guévremont, N. Duval, J. Martel-Pelletier, P. Reboul, Arthritis Res. Ther. 2007,9 , R20.

(3) J. Dion, T. Advedissian, N. Storozhylova, S. Dahbi, A. Lambert, F. Deshayes, M. Viguier, C. Tellier, F. Poirier, S. Téletchéa, C. Dussouy, H. Tateno, J. Hirabayashi, C. Grandjean, ChemBioChem 2017, 18, 2428-2440.

(4) C. G. C. Atmanene C. Ronin, S. Téletchéa, F. M. Gautier, F. Djedaïni-Pilard, F. Ciesielski, V. Vivat, Biochem Biophys Res Commun 2017, 489, 281286.

(5) N. Floyd, B. Vijayakrishnan, J. R. Koeppe, B. G. Davis, Angew. Chemie Int. Ed. 2009, 48, 7798-802.

(6) V. Percec, P. Leowanawat, H.-J. Sun, O. Kulikov, C. D. Nusbaum, T. M. Tran, A. Bertin, D. a Wilson, M. Peterca, S. Zhang, N. P. Kamat, K. Vargo, D. Moock, E. D. Johnston, D. A. Hammer, D. J. Pochan, Y. Chen, Y. M. Chabre, T. C. Shiao, M. Bergeron-Brlek, S. André, R. Roy, H.-J. Gabius, P. a Heiney, J. Am. Chem. Soc. 2013, 135, 9055-77.

(7) W. A. Bonner, J. E. Kahn, J. Am. Chem. Soc. 1951, 73, 2241-2245.

(8) K. Bock, C. Pedersen, J. Chem. Soc., Perkin Trans. 2 1974, 293-297.

(9) B. Kang, P. Okwieka, S. Schöttler, S. Winzen, J. Langhanki, K. Mohr, T. Opatz, V. Mailänder, K. Landfester, F. R. Wurm, Angew. Chemie - Int. Ed. 2015, 54, 7436-7440.

(10) Y. C. Ko, C. F. Tsai, C. C. Wang, V. M. Dhurandhare, P. L. Hu, T. Y. Su, L. S. Lico, M. M. L. Zulueta, S. C. Hung, J. Am. Chem. Soc. 2014, 136, $14425-14431$.

(11) G. J. L. Bernardes, D. P. Gamblin, B. G. Davis, Angew. Chem. Int. Ed. Engl. 2006, 45, 4007-11.

(12) P. Shu, J. Zeng, J. Tao, Y. Zhao, G. Yao, Q. Wan, Green Chem. 2015, 2545-2551.

(13) M. B. Haque, B. P. Roberts, D. A. Tocher, J. Chem. Soc. Perkin Trans. 1 1998, 2881-2890.

(14) H. P. R. Mangunuru, J. R. Yerabolu, G. Wang, Tetrahedron Lett. 2015, 56, 3361-3364.

(15) G. J. L. Bernardes, E. J. Grayson, S. Thompson, J. M. Chalker, J. C. Errey, F. El Oualid, T. D. W. Claridge, B. G. Davis, Angew. Chemie - Int. Ed. 2008, 47, 2244-2247.

(16) J. Neumann, J. Thiem, European J. Org. Chem. 2010, 900-908.

(17) M. R. J. Vallée, L. M. Artner, J. Dernedde, C. P. R. Hackenberger, Angew. Chemie - Int. Ed. 2013, 52, 9504-9508.

(18) B. Paul, W. Korytnyk, Carb Res 1984, 126, 27-43.

(19) F. Santoyo-Gonzalez, C. Uriel, J. A. Calvo-Asin, Synthesis (Stuttg). 1998, 1787-1792.

(20) G. W. Langley, A. Brinkø, M. Münzel, L. J. Walport, C. J. Schofield, R. J. Hopkinson, ACS Chem. Biol. 2016, 11, 755-762. 